\title{
Dissertation
}

\author{
submitted to the \\ Combined Faculties of the \\ Natural Sciences and Mathematics \\ of the \\ Ruperto Carola University of Heidelberg \\ for the degree of \\ Doctor of Natural Sciences
}

submitted by

Carl R. Poelking

born in Goch

Oral examination on

9 December 2015 



\section{The (Non-)Local Density of States of Electronic Excitations in Organic Semiconductors}

\section{Referees}

Prof. Dr. Kurt Kremer

Prof. Dr. Ulrich Schwarz 
This dissertation was carried out at the

Max Planck Institute for Polymer Research 
The (Non-)Local Density of States of Electronic Excitations in Organic Semiconductors The rational design of organic semiconductors for optoelectronic devices relies on a detailed understanding of how their molecular and morphological structure condition the energetics and dynamics of charged and excitonic states. Investigating the role of molecular architecture, conformation, orientation and packing, this work reveals mechanisms that shape the spatially resolved densities of states in organic, small-molecular and polymeric heterostructures and mesophases. The underlying computational framework combines multiscale simulations of the material morphology at atomistic and coarse-grained resolution with a long-range-polarized embedding technique to resolve the electronic structure of the molecular solid. We show that longrange electrostatic interactions tie the energetics of microscopic states to the mesoscopic structure, with a qualitative and quantitative impact on charge-carrier level profiles across organic interfaces. The computational approach provides quantitative access to the charge-density-dependent opencircuit voltage of planar heterojunctions. The derived and experimentally verified relationships between molecular orientation, architecture, level profiles and open-circuit voltage rationalize the acceptor-donor-acceptor pattern for donor materials in high-performing solar cells. Proposing a pathway for barrier-less dissociation of charge transfer states, we highlight how mesoscale fields generate charge splitting and detrapping forces in systems with finite interface roughness. The associated design rules reflect the dominant role played by lowest-energy configurations at the interface.

Die (nicht-)lokale Zustandsdichte elektronischer Anregungen in organischen Halbleitern Die Entwicklung von organischen Halbleitern für optoelektronische Bauelemente beruht auf einem genauen Verständnis des Zusammenhangs zwischen molekularer und morphologischer Struktur sowie energetischen und dynamischen Eigenschaften von geladenen und exzitonischen Zuständen. Diese Arbeit untersucht die bestimmenden Faktoren für die räumlich aufgelöste Zustandsdichte in organischen Heterostrukturen und Mesophasen, unter Berücksichtigung von molekularer Architektur, Konformation, Orientierung und Packung. Das zugrunde liegende rechnerbasierte Verfahren kombiniert Multiskalensimulationen der Materialmorphologie mit atomistischer oder vergröberter Auflösung mit einer langreichweitig-polarisierten Einbettungsmethode zur Beschreibung der elektronischen Festkörperstruktur. Wir zeigen, dass langreichweitige elektrostatische Wechselwirkungen die Energetik von mikroskopischen Zuständen mit der mesoskopischen Materialstruktur verknüpfen, mit einem qualitativ und quantitativ signifikanten Einfluss auf Energieprofile von Ladungsträgern an organischen Grenzflächen. Das numerische Verfahren ermöglicht zudem die Berechnung der ladungsdichteabhängigen Leerlaufspannung planarer Heteroübergänge. Die abgeleiteten und experimentell verifizierten Zusammenhänge zwischen molekularer Orientierung, Architektur und Energieprofilen rationalisieren unter anderem die AkzeptorDonor-Akzeptor-Architektur für Donormaterialien in hocheffizienten Solarzellen. Mesoskopische elektrostatische Felder generieren außerdem Ladungstrennungs- und Fluchtkräfte in Systemen mit endlicher Grenzflächenrauigkeit, die als Mechanismus zur barrierefreien Dissoziation von Ladungstransferzuständen dienen können. Darauf aufbauende Designregeln berücksichtigen die wesentliche Rolle von niederenergetischen Grenzflächenkonfigurationen. 



\section{Contents}

1 Organic Electronics in a Nutshell 1

1.1 Materials .............................. 1

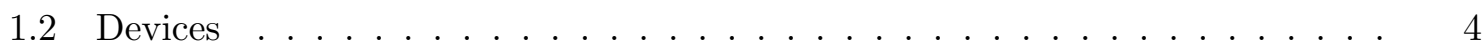

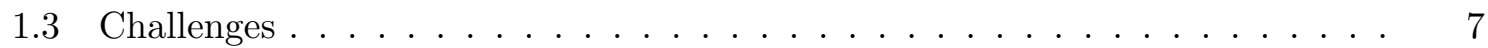

2 Particle-Based Models

of Organic Semiconductors 11

2.1 The Bottom-Up Simulation Workflow . . . . . . . . . . . . . . . . . 11

2.2 Molecular Landscapes . . . . . . . . . . . . . . . . . . . . . . . . . . . 13

2.2.1 Density Functional Theory . . . . . . . . . . . . . . . . . 14

2.2.2 Molecular Dynamics . . . . . . . . . . . . . . . . . . 17

2.2.3 Atomistic Models . . . . . . . . . . . . . . . . . . . . . . . . . . 20

2.2.4 Coarse-Grained Models . . . . . . . . . . . . . . . . 23

2.2.5 Lattice Models . . . . . . . . . . . . . . . . . . . . . . 25

2.3 Charge Transport Networks . . . . . . . . . . . . . . . . 26

2.3.1 Charge Transfer Reactions . . . . . . . . . . . . . . . . . 27

2.3.2 Graph Generation and Parametrization . . . . . . . . . . . . . . 29

2.4 Charge-Carrier Dynamics and Mobility . . . . . . . . . . . . . . . 33

3 Long-Range Polarized Embedding

of Electronic Excitations 35

3.1 The Mesoscopic Interaction Range . . . . . . . . . . . . . . . . . . . 35

3.2 The Molecular Field and Field Response . . . . . . . . . . . . . . . . . 38

3.2.1 Distributed Multipoles . . . . . . . . . . . . . . . . . . . . 39

3.2.2 Distributed Polarizabilities . . . . . . . . . . . . . . . . . 41

3.3 Long-Range Polarized Embedding _. . . . . . . . . . . . . . . . . . . . 43

3.3.1 Polarization Energy and Work . . . . . . . . . . . . . . . . 44

3.3.2 Aperiodic-Periodic Decomposition . . . . . . . . . . . . . . 45

3.3.3 Shape Terms . . . . . . . . . . . . . . . . . . . . . 48

3.4 Interaction Kernels . . . . . . . . . . . . . . . . . . . . . . . . . 51

3.4.1 Anisotropic Kernels . . . . . . . . . . . . . . . . . . . . . . 51

3.4 .2 Isotropic Kernels . . . . . . . . . . . . . . . . . . . . . . . 51

3.4 .3 Thole Damping Kernels . . . . . . . . . . . . . . . . . . . 52 
3.4 .4 Field Calculation . . . . . . . . . . . . . . . . . 52

3.5 Computational Procedure for Site-Energy Calculations . . . . . . . . . . . . 53

3.6 Summary: Long-Range Polarized Embedding . . . . . . . . . . . . . . 57

4 Charge Carriers at Organic-

ORGANIC INTERFACES

4.1 The Acceptor-Donor-Acceptor Puzzle . . . . . . . . . . . . . . . . 59

4.1.1 Level Profiles Across $\mathrm{C}_{60} /$ D5M Interfaces . . . . . . . . . . . . . 62

4.1 .2 Experimental Validation . . . . . . . . . . . . . . . . . 64

4.2 Orientation Effects and Film Patterning . . . . . . . . . . . . 65

4.2.1 Orientation Effects in Lattice Multilayers . . . . . . . . . . . 67

4.2 .2 Level Profiles in Patterned Thin Films . . . . . . . . . . . . . . . . . . 69

4.3 Charge Carriers at Donor-Acceptor Heterojunctions . . . . . . . . . . . . 71

4.3.1 Interplay of Molecular Architecture, Packing and Orientation . . . . . 71

4.3.2 The Charge-Density-Dependent Open-Circuit Voltage . . . . . . . . . 77

4.4 Charge Carriers at Grain Boundaries . . . . . . . . . . . . . . . . . . 79

4.5 Summary: Interface Energetics . . . . . . . . . . . . . . . . . . 82

5 Charge Carriers in Disordered

BulK Mesophases 83

5.1 Charge Carriers in Amorphous Dipolar Semiconductors _ . . . . . . . . . 83

5.2 Charge Carriers in Polymeric Semiconductors . . . . . . . . . . . . . . 86

5.2.1 Partially Ordered Mesophases of Poly(3-Hexylthiophene) . . . . . . 87

5.2.2 Formation of Low-Energy States and Correlations . . . . . . . . . . 88

5.3 Summary: Mesophase Energetics . . . . . . . . . . . . . . . . . . . . 92

6 Charge Transfer States at DonorAcceptor Heterojunctions 95

6.1 Pathway for Charge Splitting and Detrapping . . . . . . . . . . . . . 95

6.1.1 Dielectric Solvation of Electron-Hole Pairs . . . . . . . . . . . . . . 97

6.1.2 The Role of Interfacial Defects . . . . . . . . . . . . . . . . 100

6.1.3 Charge Push-Out Forces at Rough Interfaces . . . . . . . . . . . . . . 103

6.2 Interface Polarity versus Photovoltaic Gap . . . . . . . . . . . . . . . 107

6.3 Charge Transfer Energetics and Open-Circuit Voltage . . . . . . . . . . . . 109

6.4 Summary: Charge Transfer Energetics . . . . . . . . . . . . . . . . . . 113

7 Conclusions \& Outlook 115

$\begin{array}{ll}\text { ACKnowledgements } & 121\end{array}$

$\begin{array}{ll}\text { List of Publichtions } & 123\end{array}$

$\begin{array}{ll}\text { BIBLIOGRAPHY } & 125\end{array}$ 


\section{Chapter 1}

\section{Organic Electronics IN A NutshelL}

The discovery of conductive polymers in the 1970s [1, 2] - rewarded with the Nobel prize in chemistry in the year 2000 - and subsequent development of the first polymeric electronic devices in the 1980s [3-5] have marked the beginnings of a vast interdisciplinary research field referred to as organic electronics. Drawing from both polymeric and small-molecular semiconductors, organic thin-film transistors, solar cells, light-emitting diodes, photodetectors and sensors name just a few out of many applications that make use of the supreme chemical versatility and mechanical flexibility of the underlying "soft" molecular materials. Furthermore enabling low-temperature solution-based processing, printing and spray coating, organic electronics is set to complement the conventional silicon-based electronics in diverse ways adding functionality and improving sustainability. This introductory chapter will provide a short review of the materials and device physics, as well as of new trends and challenges that emerged in the field over the past decade.

\subsection{Materials}

Organic semiconductors are molecular materials that exist at the interface between organic, "soft" matter and semiconducting, "hard" matter: Their mechanical properties and structural variability are reminiscent of the former, their electronic properties of the latter. Based on the type of their conjugated molecular building blocks, they are broadly categorized into small-molecular [6] vs. polymeric semiconductors [7]. The associated large compound space (Table 1.1 only lists a very small selection) presents a challenge for rational compound design in general and synthetic chemistry in particular, as already small changes in the backbone and side-chain structure, side-chain length [8-10], and, for polymers, regioregularity and molecular weight $[11,12]$ may significantly alter the macroscopic material properties.

The conjugated architecture, however, is shared by all compounds, and becomes manifest in the $\pi-\pi$ interactions that result from the intermolecular van-der-Waals attraction between 
conjugated molecular planes. These should be contrasted with the far stronger intramolecular covalent interactions, which nevertheless allow for soft bonded degrees of freedom, notably torsional motion along the backbone and side chains. As a result, structure formation is governed by both energy and entropy, giving rise to complex morphologies that incorporate amorphous, liquid-crystalline and crystalline ordering.

To increase morphological control and reduce batch-to-batch variability, various processing techniques have been developed. Solution-based approaches [13], suited for both small molecules and polymers with solubilizing side chains, include spin and spray coating, solutioncoating with fluid control, epitaxy, solvent-annealing and nucleation techniques - to just name a few. Vacuum processing [14], by contrast, is only applicable to small-molecular semiconductors, where vapour-phase deposition enables the fabrication of high-purity thin films with good control over thickness and chemical composition.

Notwithstanding the morphological complexity accessed through processing, the material properties of the molecular solid tend to correlate strongly with the molecular gas-phase properties (a further consequence of the weak intermolecular - as opposed to intramolecular - interactions). Fast, intramolecular vibrations, for example, persist in the phononic spectra of the solid as modes with vanishing dispersion and energies on the order of $0.1 \mathrm{eV}$, next to the intermolecular acoustic and optical phonon modes with lower frequencies of up to several $\mathrm{THz}(0.01 \mathrm{eV})[15]$.

The molecular character of organic semiconductors also substantially determines their electronic properties: Here, the weak intermolecular coupling often prevents a quantummechanical delocalization of charge carriers and excitons beyond the intramolecular scale. This incomplete delocalization results in a non-adiabatic transport mechanism [16], where charge and energy transfer occur via "hopping" of charge carriers and excitons, respectively, between molecular localization sites. The hopping is temperature-activated and triggered by nuclear motion, with the associated electron-phonon coupling giving rise to the smallpolaron picture of charge transport with reorganization energies of $0.1-0.3 \mathrm{eV}\left(5-12 \mathrm{k}_{\mathrm{B}} \mathrm{T}\right.$ at room temperature). The electronic coupling that mediates the transfer derives from the wave-function overlap of the frontier orbitals characterized by extended $\pi$-systems of $s p^{2}$ hybridized carbons. Typical electronic coupling strengths are on the order of $0.01-0.001 \mathrm{eV}$. As this coupling increases, charges delocalize more, up to a regime where the hopping mechanism gradually ceases and band transport, as associated with inorganic semiconductors, sets in [17]. The hopping picture, however, proves a useful description in compounds with charge-carrier mobilities below $1 \mathrm{~cm}^{2} / \mathrm{Vs}[18]$ - and hence in the vast majority of materials used in organic solar cells and light-emitting diodes.

The non-adiabatic transport mechanism translates into a characteristic dependence of the charge-carrier mobility on temperature, charge density and electric field. This dependence follows from a density of states shaped by the interaction of the strongly localized charge carriers with the molecular environment. The spatially fluctuating molecular fields to which the charge carriers are hence subjected effect a Gaussian broadening $\sigma$ of the density of states on the order of $0.05-0.2 \mathrm{eV}$. The mobility is extremely sensitive to this energetic dis- 


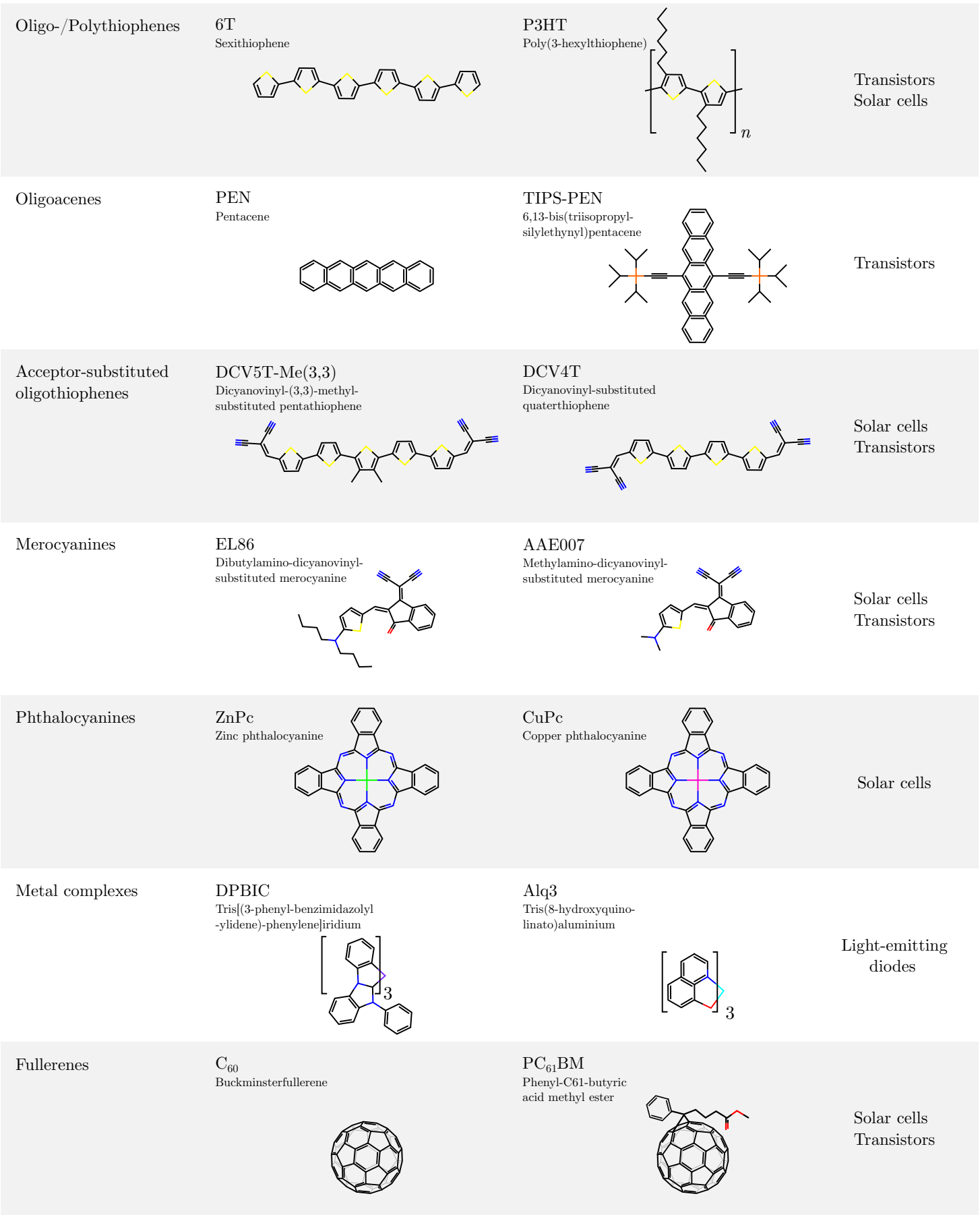

Table 1.1. Families of organic semiconductors studied in this work. Material classes (left column) and their typical applications (right column), next to the chemical structures of several small-molecular representatives, as well as the polymeric P3HT. Colour coding of the atomic elements is as follows: carbon (black), nitrogen (blue), oxygen (red), aluminium (turquoise), silicon (orange), sulfur (yellow), copper (pink), zinc (green), iridium (purple). 
order: Low-lying energy states in the density act as traps and thus limit the charge-carrier dynamics. For moderate energetic disorder, the resulting non-dispersive transport regime is approximately characterized by a mobility $\mu \sim \exp \left(-\alpha \hat{\sigma}^{2}+\beta \sqrt{F}\right)$ with effective disorder $\hat{\sigma}=\sigma / k_{\mathrm{B}} T$ and electric-field strength $F[19]$. The exponential dependence on $\sqrt{F}$ with temperature-dependent slope $\beta$ is known as the Poole-Frenkel behaviour that results from field-enhanced hopping in a spatially correlated density of states. The mobility also exhibits an exponential dependence on charge-carrier density due to the population of low-energy states and passivation of traps [20,21]. We note, however, that the intrinsic charge-carrier density in organic semiconductors is virtually zero due to the large transport gap of $2-3 \mathrm{eV}$ (this is the gap between the mean of the conduction and valence density of states). As a result, a finite charge-carrier density (and hence conductivity) has to be achieved through either molecular doping, voltage gating, photogeneration or injection of charges from external contacts.

Beyond charge carriers, the optoelectronic properties of organic semiconductors are determined mostly by Frenkel and charge transfer (CT) excitons, both of which play a key role in solar cells and light-emitting diodes. Frenkel excitons are either generated through photon absorption or formed after recombination of a CT state (i.e., an electron-hole pair state related to the ground state $S_{0}$ by a charge transfer reaction). Whereas CT states are stationary, Frenkel excitons diffuse, thus allowing for energy transport. The kinetics of the energy transfer reaction are appropriately described by a Förster rate $\sim 1 / R^{6}$ in the case of weak excitonic coupling. The exciton diffusion length is, however, limited by radiative and non-radiative recombination processes: These include fluorescence in the case of singlet $\left(S_{n}\right)$ and spin-orbit-mediated phosphorescence in the case of triplet $\left(T_{n}\right)$ states, as well as non-radiative quenching at defects, notably charge-carrier traps [22] .

\subsection{Devices}

The wealth of applications for which organic semiconductors have been suggested is dazzling. Drawing from their optical, electronic and mechanical properties, these include the "conventional" device forms known from the inorganic world (light-emitting diodes, photovoltaic cells and thin-film transistors) but extend to previously unseen devices, such as pressure- and flexion-sensitive electronic skin [23], chemical sensors for artificial noses [24], or biomedical sensors [25] in the form of disposable electronic band-aid. Many of these applications allow for solution-based processing, with the prospect, for example, of integrated circuits for memory and logic devices printed on transparent, flexible substrates.

Here we will focus on optoelectronic devices, including organic light-emitting diodes (OLEDs) and photovoltaics (OPV). OLEDs are the first and so far only organic electronic devices to have successfully entered the commercial market on a larger scale. They are by now routinely used in active-matrix OLED displays, and in the future will additionally join the lighting market. OPV, by contrast, cannot yet compete with the existing inorganic tech- 


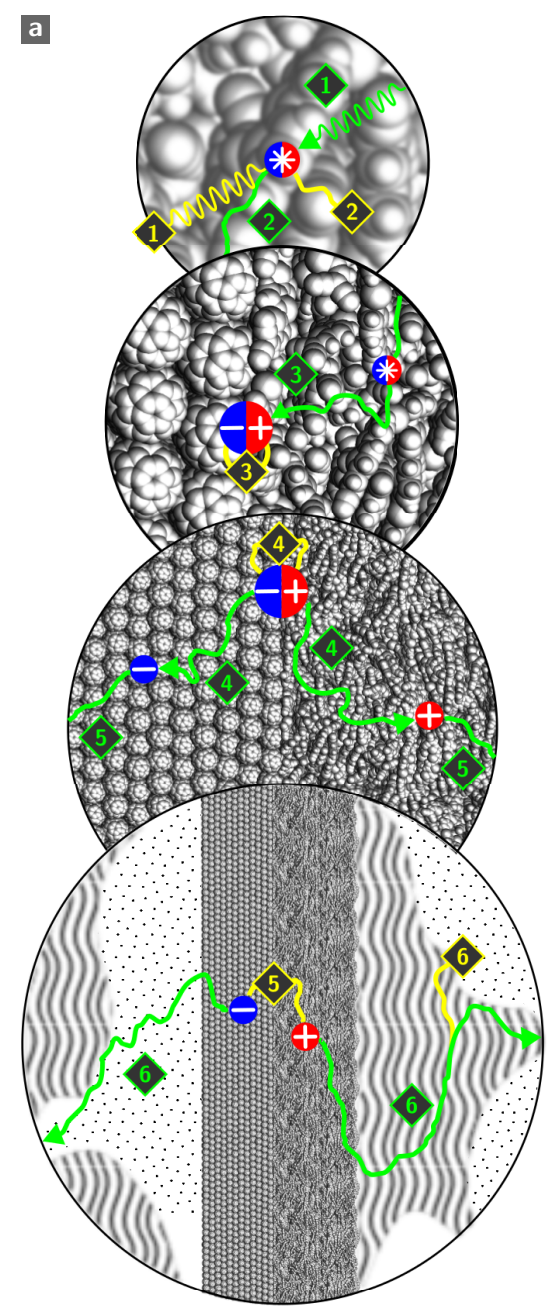

b Gain processes

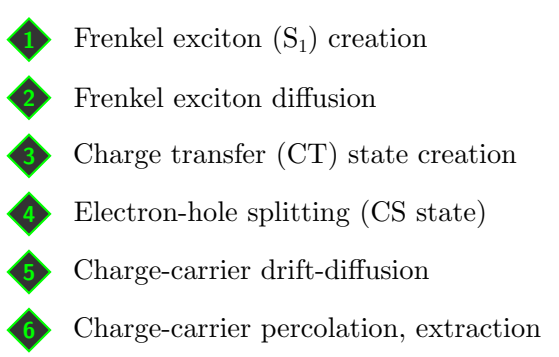

c Loss processes
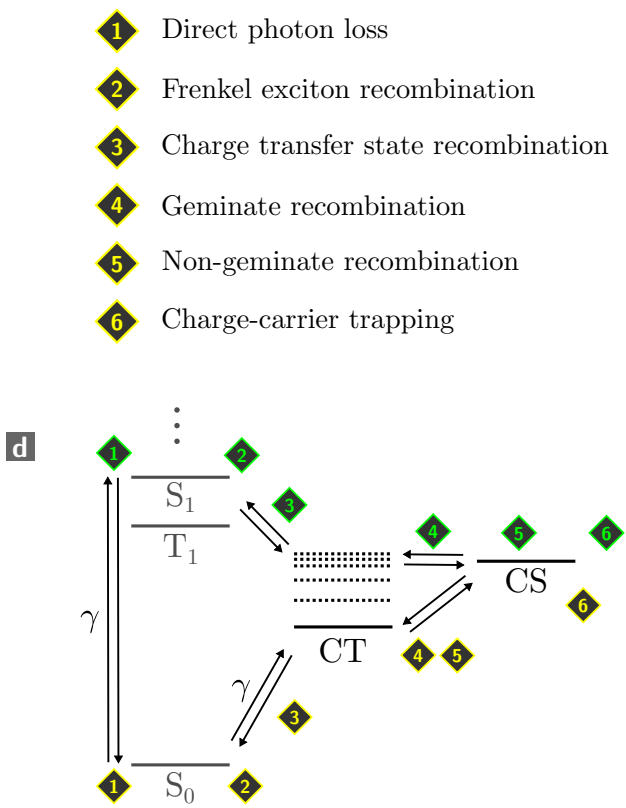

Figure 1.1. Charge generation and extraction in organic solar cells. (a) Elementary processes in organic solar cells across different length scales (from $1 \mathrm{~nm}$ to $100 \mathrm{~nm}$ ) split according to (b) gain and (c) loss processes. (d) Jablonski representation of the energetics and dynamics with ground state $S_{0}$, singlet (triplet) exciton $S_{1}\left(T_{1}\right)$, charge-transfer and charge-separated state, CT and CS, respectively. 
nologies due to low efficiencies and high-cost processing if compared to the highly optimized silicon or cadmium-telluride-based technologies. In fact, the charge generation process in OPV is still not completely understood microscopically, raising the question whether - and if so, which - fundamental physical mechanisms restrict its efficiency to below the thermodynamic limit established for inorganic cells. Nevertheless, the field has come a long way since the first report of a bilayer organic solar cell in 1986 [4]: By now, power conversion efficiencies of up to $8 \%$ for single-junction [26] and $12 \%$ for multi-junction devices [27] have been reported, even if one excludes hybrid systems such as dye-sensitized or perovskite-based solar cells.

The working principle of OPV relies on the dissociation of an optically created Frenkel exciton into free charges, i.e., an electron and a hole. The simplest device structure therefore consists of a molecular absorber layer sandwiched between a high-work-function anode and low-work-function cathode. Such a setup will, however, result in efficiencies well below $1 \%$ due to the strong binding energy of excitons in organic semiconductors: In practice, the active layer has to incorporate a heterojunction between a molecular donor with an in comparison low ionization energy and an acceptor with a low electron affinity. Excitons created on either of the two species can then dissociate via an interfacial CT state into a hole localized on the donor and electron localized on the acceptor. To be dissociated, however, the exciton first has to reach the interface through diffusion. Improving on a donor-acceptor bilayer (i.e., a planar heterojunction), an intermixed layer (bulk heterojunction) increases the percentage of excitons that reach the interface within their diffusion lengths as dictated by their lifetime. Subsequently, charge extraction to the electrodes occurs via drift-diffusion of charges through percolating domains.

Fig. 1.1a summarises these microscopic mechanisms from exciton creation to charge extraction, distinguishing between gain (Fig. 1.1b) and loss (Fig. 1.1c) processes. The primary loss mechanisms result from the incomplete spectral coverage of the absorber material, recombination of excitons and geminate $\mathrm{CT}$ states, recombination of non-geminate charge pairs, or charge-carrier trapping. The energetics that underlie these processes are in part controversial, in particular with respect to the energy of the CT state, which is thought to be coulombically bound by around $0.3 \mathrm{eV}$ with respect to the charge-separated (CS) state - if not vibrationally excited (see the Jablonski diagram in Fig. 1.1d).

For OLEDs, the thermodynamic inverse of solar cells, the same processes apply, but in reverse order: Electron and hole currents are injected from the cathode and anode respectively, and fed into a host:guest emission layer. The guest molecules are metal-coordinated complexes that act as recombination centres for triplet excitons, formed either directly on the guest or after an energy transfer via one of the host neighbours.

The dynamics and energetics of these microscopic processes eventually give rise to the macroscopic device characteristics; in OPV, these are the open-circuit voltage $V_{\text {oc }}$, shortcircuit current $J_{\mathrm{sc}}$ and fill-factor - in OLEDs, the external spectral light yield vs. input electrical power. Optimization of these observables happens both on the level of chemical design and device architecture. Today's stack architectures consist of multiple layers (see 

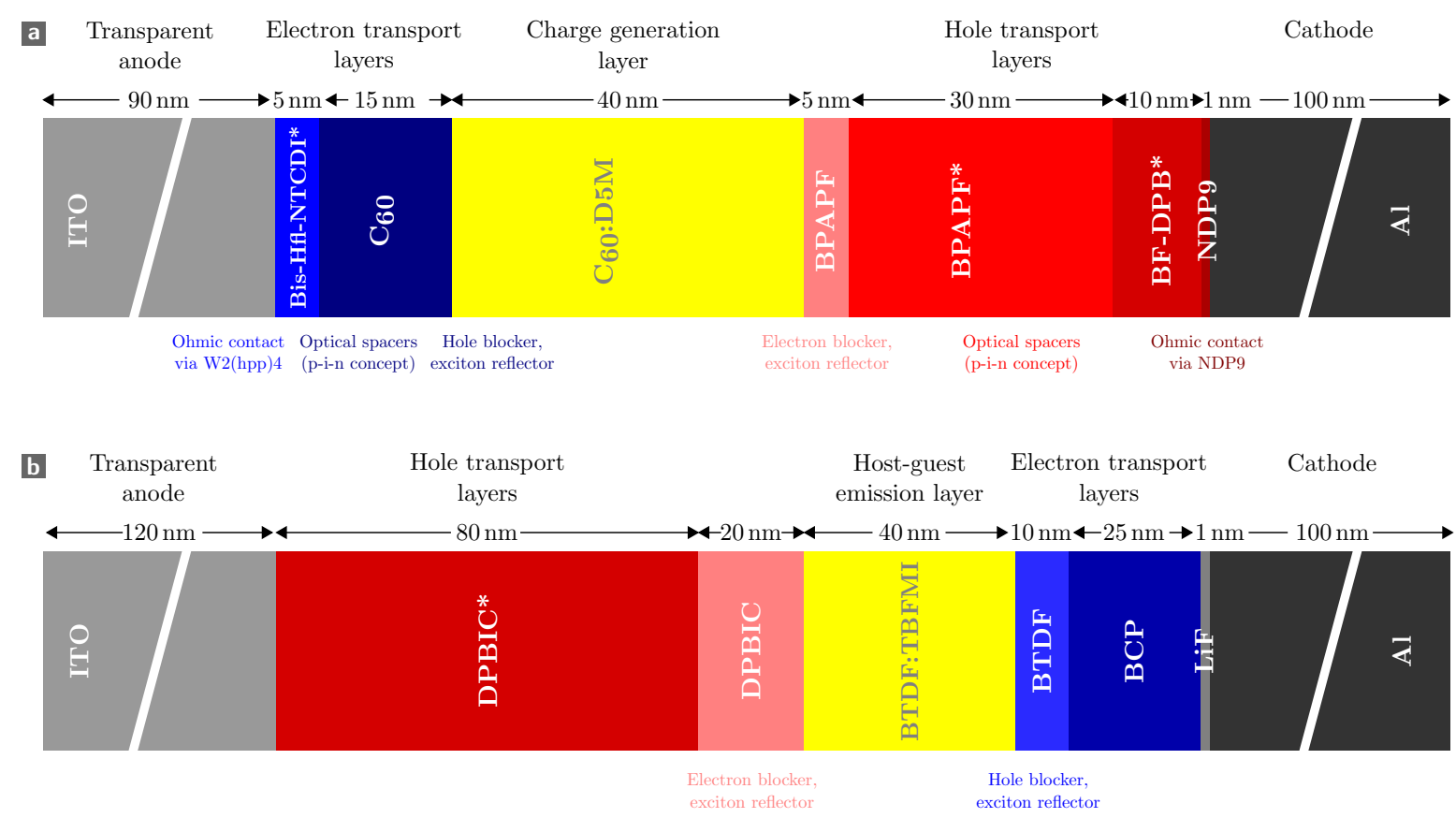

Figure 1.2. Architecture of organic optoelectronic devices. (a) Stack of the $8.3 \%$ efficient world-record OPV system $\mathrm{C}_{60}$ :D5M [26]. (b) Stack of a blue phosphorescent BTDF:TBFMI OLED [28]. Doped layers are indicated by a star $*$. Layer thicknesses are drawn to scale.

Fig. 1.2a,b) each with a specific function, including doped hole and electron transport layers, charge and exciton blocking layers or electrode contact modifiers. The layer thicknesses are optimized such as to maximize the light intensity across the active layer in OPV or enhanced light outcoupling in OLEDs. The total device thicknesses amount to on the order of hundreds of nanometers, which in the case of OPV is breathtakingly thin compared to silicon-based devices, but made possible here by the high optical density of the absorber materials. Finally, yet more elaborate stacks are required where several active layers are employed to achieve better spectral coverage: This is the case in tandem (multi-junction) solar cells on the one hand and white OLEDs on the other hand.

\subsection{Challenges}

To understand the interplay between morphological and electronic properties across multiple length scales and to link them to the macroscopic device characteristics is the key challenge in organic electronics. This requires advanced techniques for chemical synthesis, morphological control, material and device characterization.

On the material side, the synthesis of "stiff" molecules and polymers exempt from torsional defects targets enhanced charge-carrier dynamics and delocalization [29]. Additionally, new design paradigms such as the donor-acceptor (DA) or acceptor-donor-acceptor (ADA) 
architecture for small molecules, oligomers and polymers aim for stronger optical absorption both in the visible and infrared region [30-32]. Long-wavelength absorbers furthermore assist efforts to integrate exciton fission as a single-photon multi-exciton generation process into OPV, with the perspective to go beyond the Shockley-Queisser limit for single-junction devices $[33,34]$.

Tackling another pervasive issue in organic electronics, research is being undertaken to increase the chemical stability of the molecular constituents. Severe stability issues are most prominent in OLEDs, but in principle extend to any device, notably organic thin-film transistors with high-mobility n-type conductors [35]. Deep-blue emitters for OLEDs, for example, are still vulnerable to degradation processes related to the long lifetime of the highly energetic excited triplet state [36]. In this context, thermally activated delayed fluorescence is a mechanism that repopulates singlet via triplet states [37], thus not only maintaining high external quantum efficiencies, but also rendering heavy metal complexation for spin-orbit coupling redundant. The triplet-to-singlet conversion rate may, however, still be too slow to effectively prevent degradation and maintain high efficiencies at high luminances.

In an attempt to improve sustainability and mechanical flexibility, alternatives for the transparent electrode material ITO (indium tin oxide) are explored [38]. Similarly, nonfullerene acceptors for organic solar cells offer a perspective to reduce fabrication costs hoisted by the expensive synthesis of the fullerene component [39].

Beyond the synthesis of new materials, advanced processing techniques promise improved morphological control [40]: These include nucleation agents to adjust crystallite sizes [41], molecular design motifs to tune the directional alignment of polymer chains [42] and fluidcontrolled solution coating to direct crystal alignment and patterning [43].

In the context of organic solar cells, the primary challenge is, however, of a fundamental character: Namely, to understand the relationship between the energetics and dynamics of charge-transfer and charge-separated states and how they translate into the open-circuit voltage. Without molecular-level insight into how chemical and morphological features jointly shape the densities of states of the relevant excitations, rational compound design of new donor and acceptor materials remains difficult. Nevertheless, considerable experimental and theoretical efforts have been made to elucidate structure-property relationships of this type. On the experimental side, diverse approaches investigate and correlate the (1) energetics via, e.g., ultraviolet, inverse and two-photon photoemission spectroscopy, (2) dynamics via transient-absorption spectroscopy, charge-extraction techniques or light-induced electron-spin resonance and (3) structural properties via electron or X-ray diffraction and scattering, atomicforce microscopy, infrared ellipsometry, nuclear magnetic resonance, as well as many other techniques.

As a rule, the complex structure of organic semiconductors significantly complicates the interpretation of the thus obtained experimental data. Theoretical and computational models have therefore proven valuable in rationalizing material and device properties, using, for example, top-down approaches such as Gaussian disorder or drift-diffusion models tailored to the description of charge and energy transport [19, 20, 44, 45]. These mesoscopic models 
lack, however, a systematic link to the structural features on a molecular scale, which is a prerequisite for in-silico compound screening. Quantum-mechanical methods that describe electronic processes on the level of isolated molecular pairs in turn fail to account for the influence of morphological features on the mesoscopic scale [46].

In this thesis, we will present a bottom-up simulation framework that addresses the relationship between the morphology and energetics in organic semiconductors in a quantitative manner and with a systematic link to the molecular and supramolecular structures. We will focus in particular on the effect of molecular architecture, packing and mesoscopic organization on the energetics of charge carriers and CT states at organic-organic interfaces and in partially ordered mesophases.

The outline is as follows: In Chapter 2, we will detail the workflow for microscopic, particle-based simulations of the molecular and electronic structure of organic semiconductors. Further in this direction, Chapter 3 describes a long-range polarized embedding procedure as a perturbative approach tailored to the calculation of the spatially resolved (local) density of states of molecular materials. The thus developed techniques will be applied to the study of charge carriers in organic-organic heterostructures and partially ordered mesophases in Chapters 4 and 5, respectively. We will show how the mesoscopic organization at donoracceptor heterojunctions governs level profiles, taking into account molecular architecture, packing and orientation. By evaluating the thermally-broadened density of states for a variety of molecular donor materials, we will indicate how the charge-density-dependent open-circuit voltage follows from the thin-film energetics in a mesostructured morphology. Additionally, studies of patterned thin films and grain boundaries will highlight the different character of in- and out-of-plane interfaces, as well as the role played by low-energy packing modes in the prevention of energetic traps at these boundaries. In Chapter 5, it will be shown how simulations of polymeric mesophases with varying degree of liquid-crystalline order resolve the formation of low-energy states and extended spatial correlations in the density of states with increasing structural order. Finally, Chapter 6 will focus on the role of CT energetics at donor-acceptor interfaces, with particular emphasis on how CT energies can be controlled through interface engineering to drive charge separation. The associated charge splitting and detrapping forces imply orientational constraints and energetic tradeoffs for functional donoracceptor interfaces. Finally, Chapter 7 will conclude the work with suggestions for method extensions and future research. 


\section{Chapter 2}

\section{PARTiCle-Based Models of Organic SEMiconductors}

A core challenge in computational materials science is the prediction of material properties from first principles and hence - in the context of molecular materials - from input chemical structures. In this chapter, we present a workflow for particle-based microscopic simulations of organic semiconductors that aims to achieve just that. It is tailored to the description of non-adiabatic charge transport. The workflow can be broken down into three steps: First, the material morphology is simulated at an atomistic level, using molecular dynamics or other particle-based models, including coarse-graining and backmapping techniques. Second, a charge transport network is constructed from the simulated morphology, built on a rate-based description, where the parametrization of the rates is achieved on a quantum or quantumclassical level. Third, kinetic Monte-Carlo simulations are used to derive macroscopic observables, notably charge-carrier mobilities, or simulate current-voltage characteristics of realistic devices either directly or via parametrization of continuous drift-diffusion models.

\subsection{The Bottom-Up Simulation Workflow}

The functional properties of organic semiconductors arise from structural features and dynamic processes across many length and time scales. On the structure side, local molecular packing $\left(10^{-9} \mathrm{~m}\right)$, domain organization $\left(10^{-8} \mathrm{~m}\right)$ and device-level architecture $\left(10^{-7} \mathrm{~m}\right)$ all have an impact on device characteristics. The same applies to the time domain: Nuclear vibrations $\left(10^{-15} \mathrm{~s}\right)$, charge transfer dynamics $\left(10^{-15}-10^{-9} \mathrm{~s}\right)$, molecular diffusion $\left(10^{-9} \mathrm{~s}\right)$ and charge migration $\left(10^{-9}-10^{-6} \mathrm{~s}\right)$ necessitate a similarly multiscale picture. With today's computational resources, there is no simulation technique available that can possibly address all these length and time scales simultaneously. For practical computations, approximations based on a separation of these scales are inevitable.

In organic semiconductors (and soft matter in general) an at least partial separation of time and length scales derives from the difference in strength of the interactions between 


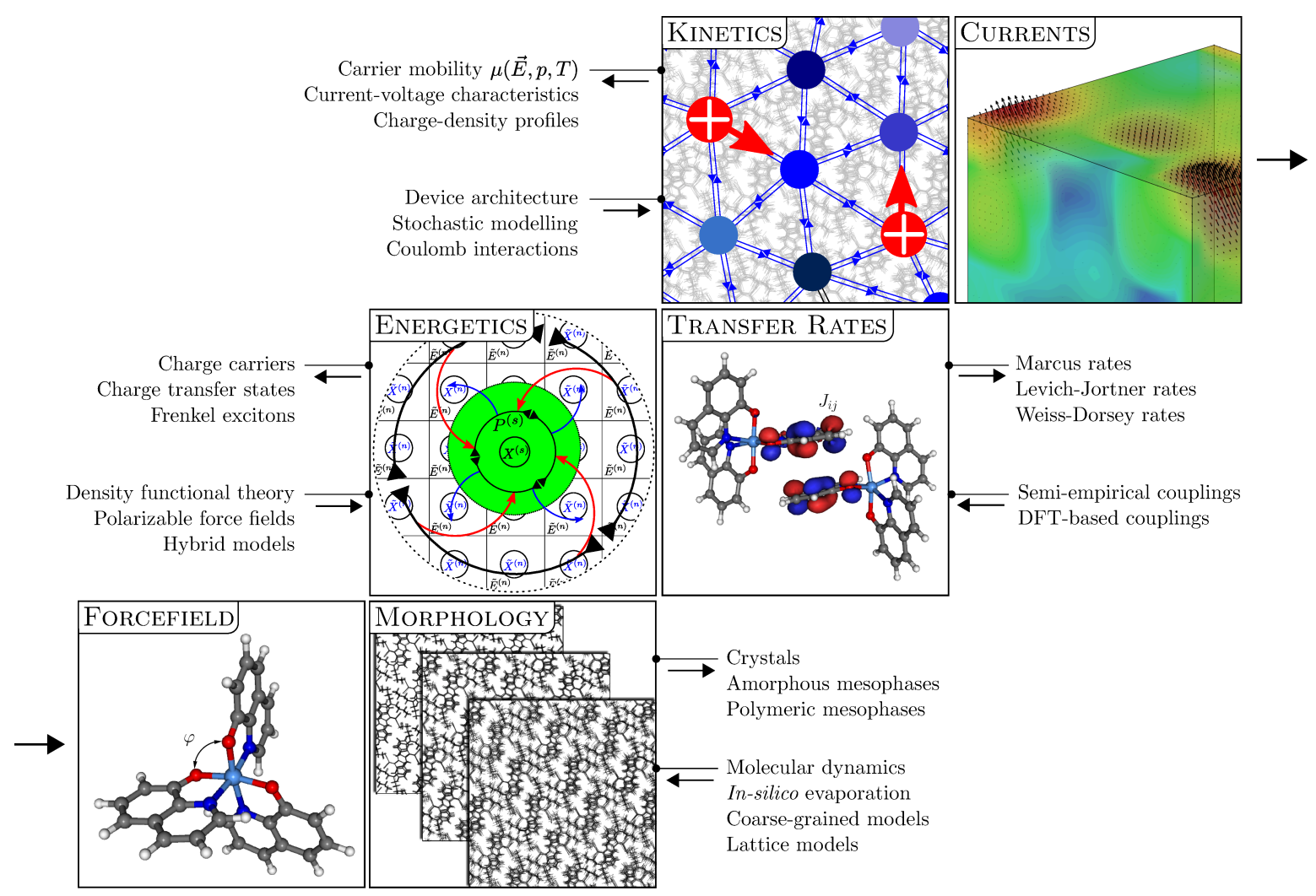

Figure 2.1. Bottom-up workflow for charge and energy transport simulations. The schematic summarizes the three-level procedure as implemented in the VOTCA software suite. Bottom: Particle-based simulation of the material morphology via force-field-based molecular dynamics, including atomistic, coarse-grained or lattice models. Centre: Partitioning of the morphology onto a transport network and parametrization thereof from first principles. Top: Simulation of kinetics on the charge transport network and evaluation of macroscopic mobilities and currents.

and within the molecular building blocks - different from most "hard" matter, where the extended conjugated network in inorganic semiconductors or long-range metallic binding in metals prevent any such distinction. For the problem of charge transport in organic materials, the weak intermolecular coupling implies that nuclear and electronic degrees of freedom can (to some approximation [47-49]) be decoupled in a small-polaron way: Intramolecular nuclear/vibrational degrees of freedom are then included effectively in an appropriately chosen rate expression for charge or energy transfer, such as the semi-classical Marcus rate. Molecular diffusion, on the other hand, can be entirely disentangled from the dynamics of electronic states. Electronic processes are hence thought to occur in a frozen morphology that can be precomputed.

Fig. 2.1 presents the simulation workflow associated with this separation of time scales. In the following sections of this chapter, we will work our way through the individual stages of the workflow (except for the energetics of excitations, to which we devote a separate chapter). 
The nutshell version reads as follows: First, the material morphology is simulated based on atomistic or coarse-grained force fields. This may include particle-based methods with phenomenological interactions required to address morphological scales beyond the limit set by atomistic molecular dynamics. A significant fraction of the complexity of organic materials enters at this stage due to the diverse structuring of materials, ranging from crystalline to liquid-crystalline to amorphous ordering and including domain and interface formation, grain boundaries, binary mixtures, electrodes, etc. Once the morphology has been simulated, a transport network is generated from the molecular landscapes and the associated reaction rates parametrized from first-principles-based techniques. Already at this stage, structureproperty relationships that relate morphological features to the energy landscape (as the focal point of this work) can be established. Finally, dynamics on the network can be simulated to evaluate charge-carrier or exciton mobilities, which can be further used in device simulations built on larger stochastically generated transport networks or continuous device models.

The outlined simulation protocol, which is by now routinely used for the study of charge transport in organic semiconductors, is implemented in the open-source simulation package VOTCA (Versatile Object-oriented Toolkit for Coarse-graining Applications) [50]. The modular structure of this toolkit - whose extension was an important aspect of this work - includes four sub-libraries, each devoted to a specific task in the workflow from Fig. 2.1: The coarse-graining library VOTCA::CSG allows the derivation of coarse-grained potentials for the morphology simulation. The charge-transport module VOTCA::CTP assists with the generation of the transport network and parametrization of the transfer rates, including the computation of energies of electronic states (charge carriers, charge transfer states, Frenkel excitons) and the calculation of electronic couplings. The molecular orbital overlap library VOTCA::MOO implements a semi-empirical ZINDO approach tailored to the computation of these couplings in large systems. Finally, the VOTCA::KMC module incorporates a kinetic Monte Carlo code that simulates dynamics on the generated transport network.

\subsection{Molecular Landscapes}

The morphological complexity of the molecular and polymeric solid state (characteristic of many soft matter systems) renders a unified description infeasible. Still, there are a range of computational approaches available to describe at least select features of the morphology associated with different length and time scales (see our introductory discussion). Here, we will focus on particle-based descriptions targeting system sizes up to $100 \mathrm{~nm}$, where "particles" may represent nuclei and electrons, atoms, molecular moieties or entire molecules. These should be contrasted with field-based descriptions, notably classical density functional theory based, e.g., on time-dependent Ginzburg-Landau theory. Field-based approaches are, however, less suited for charge and energy transport simulations, which rely on an atomistic representation of the system, obtained from particle-based methods either directly or after backmapping. 
In the following, we will describe how particle-based descriptions at different levels of resolution are employed to obtain realistic microscopic models of organic semiconductors. Fig. 2.2 presents an overview over the thus obtained morphologies, grouped according to system type (bulk, heterojunction, grain boundary) and resolution (atomistic, coarse-grained, lattice). We will begin with the highest level of resolution, and in doing so first introduce two computational techniques widely applied to molecular ordering at the atomistic scale: quantum-mechanical density functional theory and classical molecular dynamics.

\subsubsection{Density Functional Theory}

Density functional theory (DFT) [51] is a popular approach to study ground-state structural and electronic properties of interacting many-body systems. The underlying idea is to absorb the interaction between the particles into an energy functional of the particle density, leading to an effective description of the system in terms of non-interacting particles - thus reducing computational complexity. Quantum-mechanical DFT targets the interacting-particle problem described by a Hamiltonian of the form [52]

$$
\begin{aligned}
\hat{H}= & -\frac{\hbar^{2}}{2 m_{e}} \sum_{i} \nabla_{i}^{2}-\sum_{i, I} \frac{Z_{I} e^{2}}{\left|\boldsymbol{r}_{i}-\boldsymbol{R}_{I}\right|}+\frac{1}{2} \sum_{i \neq j} \frac{e^{2}}{\left|\boldsymbol{r}_{i}-\boldsymbol{r}_{\boldsymbol{j}}\right|}- \\
& -\sum_{I} \frac{\hbar^{2}}{2 M_{I}} \nabla_{I}^{2}+\frac{1}{2} \sum_{I \neq J} \frac{Z_{I} Z_{J} e^{2}}{\left|\boldsymbol{R}_{I}-\boldsymbol{R}_{J}\right|} .
\end{aligned}
$$

with nuclei at positions $\boldsymbol{R}=\left\{\boldsymbol{R}_{I}\right\}$, with charges $\boldsymbol{Z}=\left\{Z_{I} e\right\}$ and masses $\boldsymbol{M}=\left\{M_{I}\right\}$, and electronic coordinates $\boldsymbol{r}=\left\{\boldsymbol{r}_{i}\right\}$. The challenge in solving the Schrödinger equation for the above Hamiltonian is primarily due to the electron-electron repulsion term, i.e., the third term on the right-hand side of Eq. 2.1. The Coulomb interaction between electrons gives rise to quantum-mechanical exchange and correlation. Exchange phenomena can still be captured with a many-body wave function in the form of a single Slater determinant used in HartreeFock, whereas correlation effects, which further lower the energy of the system, require at least linear combinations of these determinants (leading to the Configuration Interaction approach). To avoid the computational difficulties associated with this complicated manybody wave function, the Kohn-Sham construction of DFT operates on an effective energy functional of the electron density $\rho$,

$$
U[\rho]=T[\rho]+\int V_{\mathrm{ext}}(\boldsymbol{x}) \rho(\boldsymbol{x}) d^{3} x+\int \frac{\rho(\boldsymbol{x}) \rho\left(\boldsymbol{x}^{\prime}\right)}{\left|\boldsymbol{x}-\boldsymbol{x}^{\prime}\right|} d^{3} x d^{3} x^{\prime}+U_{\mathrm{xc}}[\rho],
$$

with $T[\rho]$, the kinetic energy of the non-interacting effective particles, the nuclear-attraction potential $V_{\text {ext }}(\boldsymbol{x})$, the classical Hartree (self-)energy of the charge density with associated potential $V_{H}(\boldsymbol{x})$, and finally the exchange-correlation functional $U_{\mathrm{xc}}[\rho]$. The ground-state particle density follows from a variational principle: Based on the functional from Eq. 2.2, 

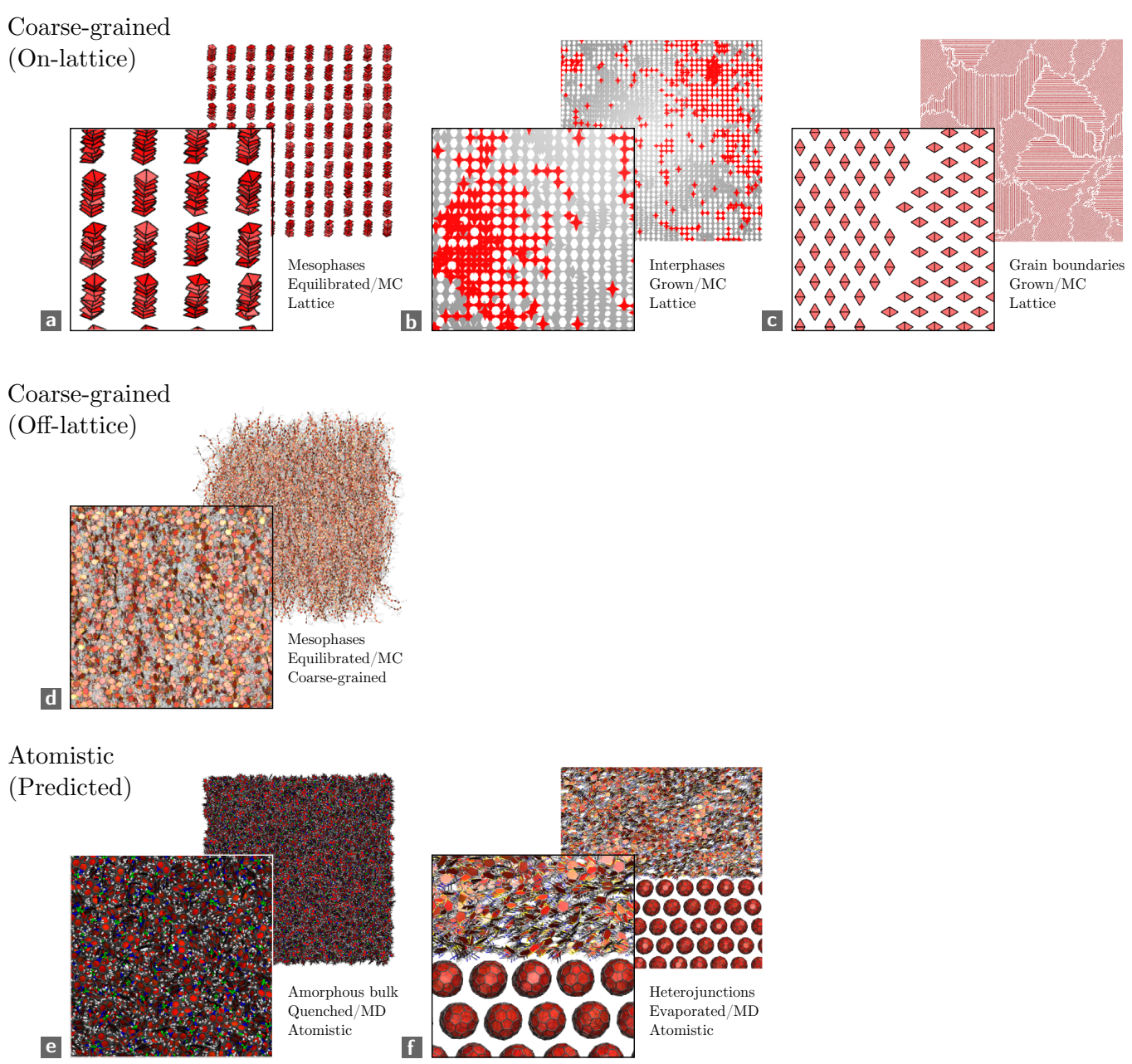

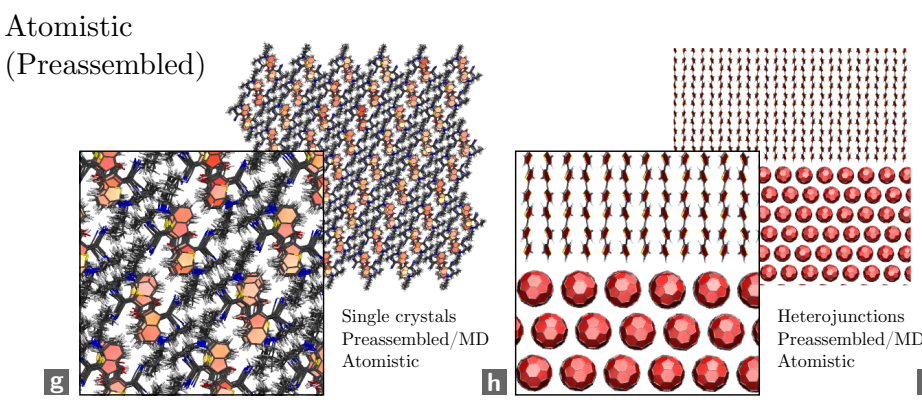

Bulk Systems

Heterojunctions

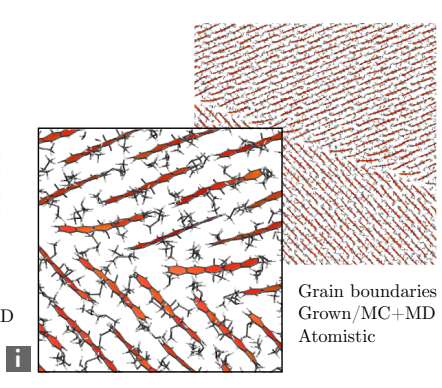

Grain Boundaries

Figure 2.2. Bulk and interfacial morphologies. Overview over lattice (a-c), coarse-grained (d) and atomistic (e-i) models of organic semiconductors studied in this work, classified according to system type (bulk, heterojunction, grain boundary). In the visualizations, $\pi$-conjugated rings are represented by red polygons. 
the Kohn-Sham eigenvalue equations result as

$$
\left[-\frac{\hbar^{2}}{2 m_{e}} \nabla^{2}+V_{\mathrm{ext}}(\boldsymbol{x})+V_{\mathrm{H}}(\boldsymbol{x})+\frac{\delta U_{\mathrm{xc}}}{\delta \rho}\right] \varphi_{i}^{\sigma}(\boldsymbol{x})=\varepsilon_{i}^{\sigma} \varphi_{i}^{\sigma}(\boldsymbol{x}) .
$$

For computational convenience, the particle density $\rho$ is expanded in terms of single-electron orbitals (basis functions) of $\operatorname{spin} \sigma, \varphi_{i}^{\sigma}(\boldsymbol{x})$ :

$$
\rho(\boldsymbol{x})=\sum_{i=1}^{N} \sum_{\sigma}\left|\varphi_{i}^{\sigma}(\boldsymbol{x})\right|^{2} .
$$

Starting from an initial guess for the density, Eqs. 2.3 and 2.4 are solved in a self-consistentfield (SCF) manner. The accuracy of the resulting density and energy greatly relies on an appropriate choice for the basis set $\left\{\varphi_{i}^{\sigma}\right\}$ and the exchange-correlation functional $U_{\mathrm{xc}}$. As the exact form of this functional is only known for the free-electron gas, various parametrizations tailored to different systems and observables - exist. Within this work, we will use the B3LYP (Becke, three parameters, Lee-Yang-Parr) hybrid functional, which has been parametrized on and shown to accurately describe ground-state energies of molecules, including ionization energies. This hybrid functional combines exact exchange from Hartree-Fock with functionals based on the local density and generalized gradient approximations (LDA and GGA), using three weighting coefficients extracted from fits to reference energies of a large set of molecules.

For the expansion of the density, we will use so-called split valence triple zeta basis sets [53] with additional polarization functions, typically denoted in the form $X-Y_{1} \ldots Y_{N} g$ : 6-311+g(p,d), for example, indicates that core electron orbitals are contracted from six primitive Gaussians, whereas valence atomic orbitals are composed of $N=3$ basis functions $\left(Y_{1} \ldots Y_{N}=311\right)$, where the first one is itself a contraction of three primitive Gaussians. Finally, additional $(p, d)$ functions of angular momentum $l=1$ and $l=2$, respectively, are added to capture polarization, next to diffuse $(+)$ functions on heavy atoms.

Due to its accuracy and computational efficiency, density functional theory is well suited to study molecular arrangements with a size of on the order of hundreds of atoms. Gas-phase properties, including ionization energies and electron affinities, can hence be calculated with relative ease. In doing so, it should be noted that orbital energies in DFT do not carry physical meaning. Hence, ionization energies and electron affinities should always be computed from two independent calculations on the neutral and charged molecule. As an example, Fig. 2.3 provides a summary of the gas-phase energetics of different DCV5T derivatives obtained by DFT with the B3LYP functional and a small (6-31g) basis set. Naturally, such calculations in gas-phase should not be used to extrapolate to the solid state. Still, they can serve as a first indicator how variation of side chains and functionalization via exchange of chemical moieties may impact energetics, here shown for the case of fluorination and methylation. Specifically, Fig. 2.3 illustrates how attachment of electronegative units (fluoromethyl) lowers the energy of the highest occupied and lowest unoccupied molecular orbitals (HOMO and LUMO, respectively), whereas electropositive groups (methyl) effect the opposite. 

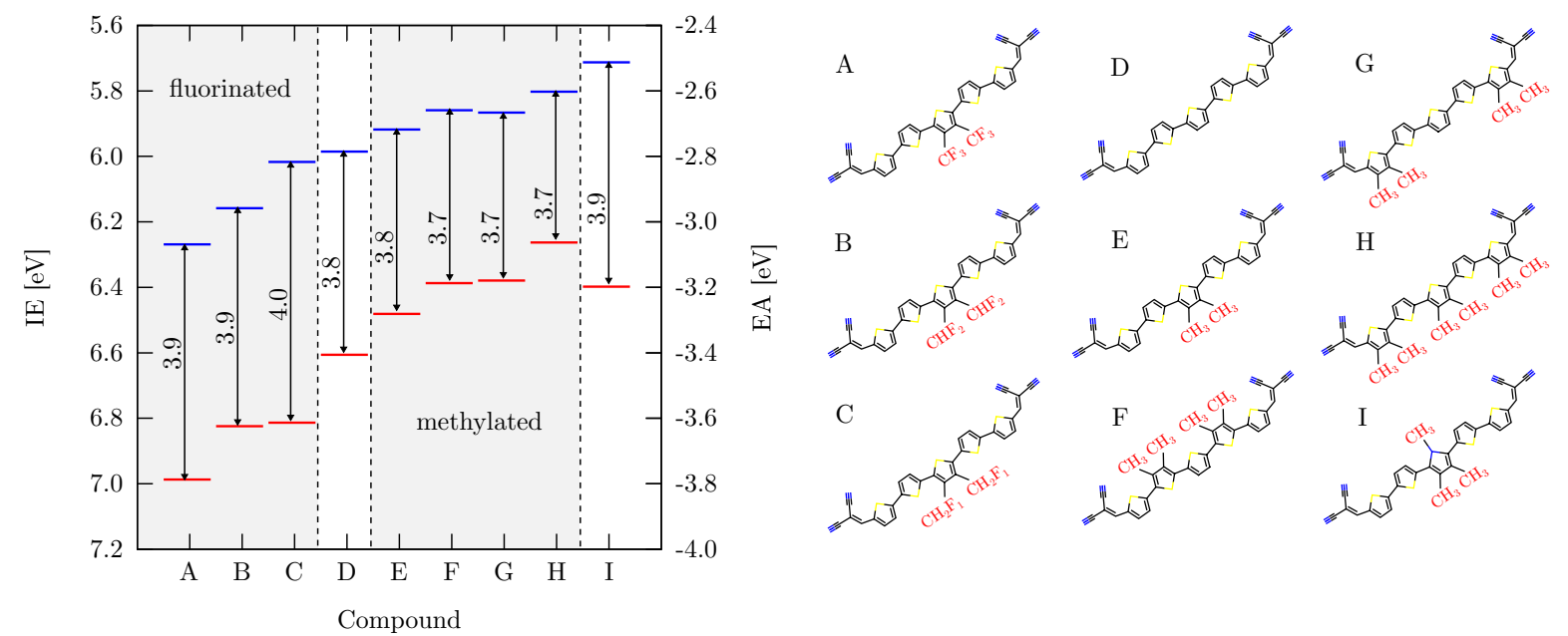

Figure 2.3. Fluorination vs. methylation of DCV5T. Gas-phase ionization energies (IE, red bars with left axis) and electron affinities (EA, blue bars with right axis) for derivatives of the acceptor-substituted oligothiophene DCV5T computed via DFT with the B3LYP functional and 6-31g basis set. Note that in compound I, the central thiophene is replaced by a pyrrole.

\subsubsection{Molecular Dynamics}

Even though DFT accurately describes molecular ground-state electronic and conformational properties, it is not suited to sample the morphology of molecular materials beyond small molecular clusters due to the associated computational complexity. For the simulation of material morphology on the scale of tens of nanometers, classical molecular dynamics (MD) [54] is the method of choice. It relies on a classical parametrization of the potential energy surface of the many-body system, obtained from first principles calculations or experimental data. Based on this force field, the Newtonian equations of motions are integrated numerically within a suitably chosen thermodynamic ensemble, using energy-conserving and time-reversible integrators as based, for example, on the leap-frog algorithm:

$$
\begin{aligned}
\boldsymbol{r}_{i}(t+d t) & =\boldsymbol{r}_{i}(t)+v_{i}(t+d t / 2) d t, \\
\boldsymbol{v}_{i}(t+d t / 2) & =\boldsymbol{v}_{i}(t-d t / 2)+\boldsymbol{f}_{i}(t) / m_{i} d t .
\end{aligned}
$$

Here, $\boldsymbol{f}_{i}(t)$ is the force acting on particle $i$ at position $\boldsymbol{r}_{i}(t)$ with velocity $\boldsymbol{v}_{i}(t)$. The main difference from other sampling techniques, notably Monte-Carlo (MC) approaches, is the explicit time coordinate $t$ along which the dynamics are simulated. Accordingly, MD approximates the ensemble average of a physical observable by its time average rather than an average over (canonically or otherwise sampled) coordinate configurations. Compared to MC, this can result in a lower sampling efficiency, for example in "simple" systems with characteristic, slow relaxation modes, but enables the simulation of non-equilibrium processes where particle velocities (giving, for instance, rise to hydrodynamic flow fields) are relevant descriptors. Fur- 
thermore, adaptations of the MD formalism such as stochastic dynamics (SD) can improve the sampling speed via a stochastic force (resembling a friction term) acting on the particles, with its time-correlation adjusted to preserve a canonical ensemble.

Typical ensembles used in MD are of the $N V T$ or $N P T$ type, the former enforcing a constant volume $(V)$, the latter a constant pressure $(P)$ next to conserved particle number $(N)$ and temperature $(T)$. Whereas particle number and system volume can be trivially fixed by the initial configuration and choice of the simulation cell, pressure and temperature control require additional coupling algorithms. The Berendsen barostat [55], for example, subjects the pressure tensor calculated from the atomistic configuration,

$$
P=\frac{1}{V}\left(\sum_{i} m_{i} \boldsymbol{v}_{i} \otimes \boldsymbol{v}_{i}+\sum_{i<j} \boldsymbol{r}_{i j} \otimes \boldsymbol{F}_{i j}\right),
$$

to a relaxation law $d P / d t \sim\left(P_{0}-P\right) / \tau_{p}$ that links the pressure change $d P$ to a target pressure $P_{0}$ according to a relaxation time $\tau_{p}$. The system compressibility relates this pressure to a volume change. Hence, the pressure relaxation is achieved by simultaneous scaling of the particle positions and simulation cell.

Whereas pressure coupling is most relevant during the initial relaxation phase of a system, temperature coupling is crucial throughout the entire simulation run. Here, despite the classical description, system sizes studied by MD are still insufficient in the sense that the explicit simulation of an appropriately sized heat bath is not only computationally prohibitive, but would furthermore result in large energy fluctuations of the coupled subsystem due to the relatively small number of contained particles (on the order of $10^{5}$ rather than the macroscopically required $10^{23}$ ). To perform simulations at constant temperature that avoid the computational overhead associated with an explicit heat bath and prevent large finite-size-induced fluctuations of the energy, a virtual heat bath (thermostat) is implemented either through direct manipulation of the particle velocity via rescaling or stochastic "kicks", or through extension of the system Lagrangian by a virtual degree of freedom that transfers energy between an implicit heat bath and the particle system. The SD formalism, for example, already implements a thermostat due to the stochastic friction term, where the coupling strength follows from the chosen friction coefficient. For MD, we will usually resort to the efficient velocity-rescaling algorithm [56] implemented in the MD package GROMACS [57]. Here, the kinetic energy spread out over $N_{f}$ degrees of freedom is allowed to fluctuate according to a canonical distribution: The change in kinetic energy for each integration step is subjected to an equation of motion, incorporating both a relaxation term that reverts the kinetic energy to its ensemble average $\frac{1}{2} N_{f} k_{\mathrm{B}} T$ with a time constant $\tau$, next to a Wiener process inherited from SD.

The quality of MD simulations greatly relies on the accuracy of the underlying force field. This force field features contributions from both bonded $(b)$ and non-bonded $(n b)$ interactions 

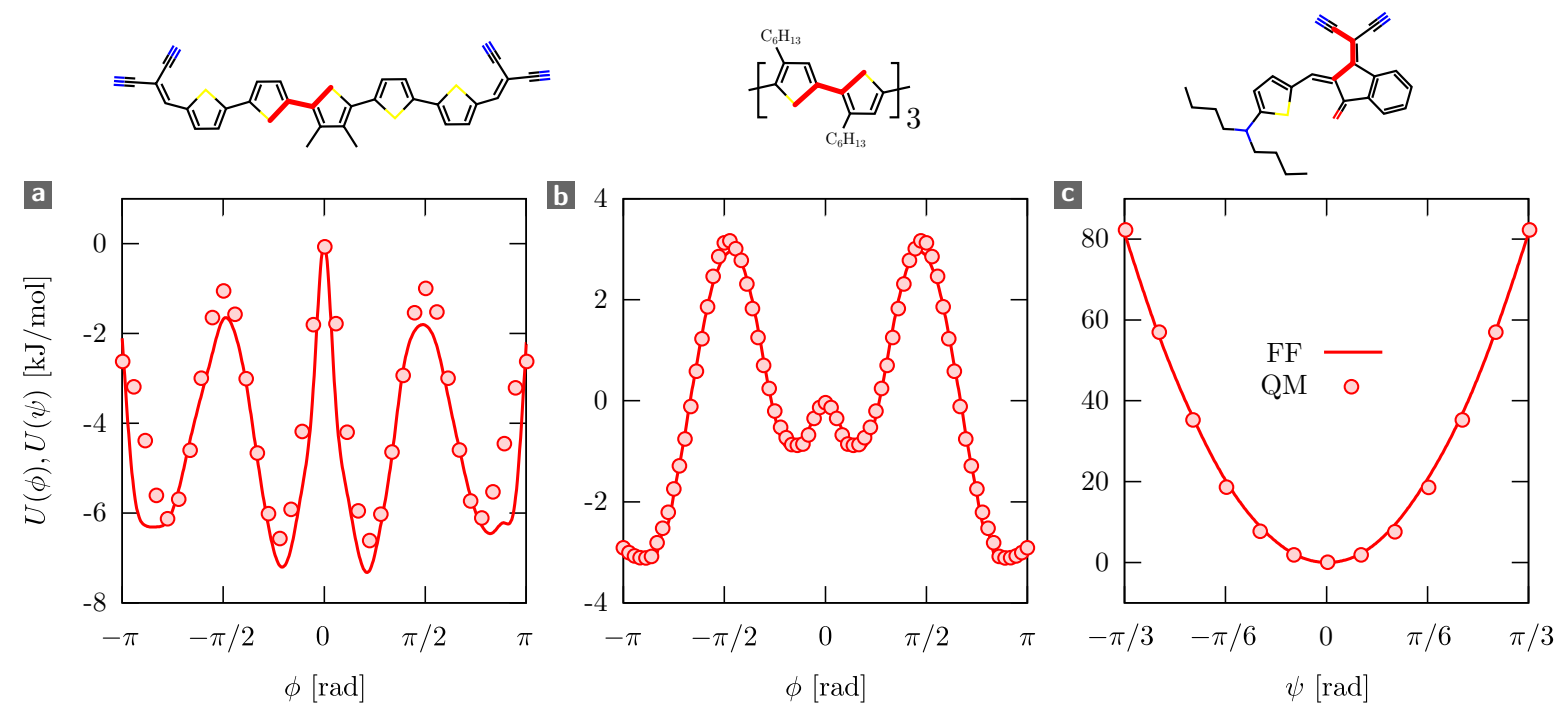

Figure 2.4. Parametrization of classical force fields. Potential energy surface (PES) resolved along the torsional degrees of freedom as highlighted (see thick red bonds) in the chemical structures of D5M (a), P3HT (b) and EL86 (c). The PES in the classical parametrization (solid lines) successfully approximates the reference PES calculated via DFT-B3LYP/6$311+\mathrm{g}(\mathrm{d}, \mathrm{p})$ (circles).

described by two-, three- and four-body potentials:

$$
\boldsymbol{f}_{i}=-\nabla_{i}\left(\sum_{<j, k>}^{(\mathrm{nb})} U_{j k}^{n b}+\sum_{<j, k>}^{(\mathrm{b})} U_{j k}^{b}+\sum_{<j, k, l>}^{(\mathrm{b})} U_{j k l}^{b}+\sum_{<j, k, l, m>}^{(\mathrm{b})} U_{j k l m}^{b}\right) .
$$

Here, non-bonded interactions absorb the coulombic and van-der-Waals, as well as Pauli exclusion interaction between particles in a pairwise approximation. For the parametrization of these non-bonded interactions, it suffices to specify atomic charge, Lennard-Jones radius $\sigma_{i}$ and well-depth $\epsilon_{i}$, provided the Lennard-Jones potential

$$
U_{\mathrm{LJ}}^{(\mathrm{nb})}\left(r_{i j}\right)=4 \epsilon_{i j}\left[\left(\frac{\sigma_{i j}}{r_{i j}}\right)^{12}-\left(\frac{\sigma_{i j}}{r_{i j}}\right)^{6}\right]
$$

is used to model the combined effect of Pauli repulsion $\left(\sim r^{-12}\right)$ and van-der-Waals attraction $\left(\sim r^{-6}\right)$ based on the combination rules $\sigma_{i j}=\sqrt{\sigma_{i} \sigma_{j}}$ and $\epsilon_{i j}=\sqrt{\epsilon_{i} \epsilon_{j}}$. Meanwhile, bonded interactions are modelled using bond (two-body), angle (three-body) and dihedral (four-body) potentials including

$$
\begin{aligned}
U_{\text {ang }}(\theta) & =\frac{1}{2} k_{\theta}\left(\theta-\theta_{0}\right)^{2}, \\
U_{\text {imp }}(\psi) & =\frac{1}{2} k_{\psi}\left(\psi-\psi_{0}\right)^{2},
\end{aligned}
$$




$$
U_{\mathrm{RB}}(\phi)=\sum_{n=0}^{5} C_{n} \cos ^{n}(\phi) .
$$

Here, $U_{\mathrm{RB}}$ is a Ryckaert-Bellemans potential which describes "soft" torsional degrees of freedom $\phi$, whereas $U_{\text {imp }}$ defines "hard" (improper) dihedrals $\psi$ with a harmonic behaviour throughout the thermally accessible range of motion. For illustration, Fig. 2.4a-c shows potential-energy surface scans associated with the backbone torsional degrees of freedom in three different compounds. The underlying potentials are either of the Ryckaert-Bellemans (Fig. 2.4a and b) or harmonic form (Fig. 2.4c). They were parametrized from reference DFT-B3LYP $/ 6-311+\mathrm{g}(\mathrm{d}, \mathrm{p})$ calculations by fitting the difference between the MD and DFT energy, the former calculated with zero force applied to the scanned degree of freedom, to the appropriate expression for the potential.

Note that in developing a force field, the differentiation between bonded and non-bonded interactions on an intramolecular level is to some degree ambiguous and has to be set by convention: The OPLS-AA [58] force field, for example, from which we will adopt LennardJones parameters, excludes non-bonded interactions between particles separated by less than three chemical bonds. Particles separated by three bonds experience a potential that is scaled down by a factor $1 / 2$. Only particles separated by more than three bonds are subjected to the full non-bonded potential.

\subsubsection{Atomistic Models}

We will now present examples for how MD is applied to the study of organic semiconductors. The aim is to develop atomistic models that can subsequently be used for electronic structure calculations. As MD is limited to system sizes of on the order of $10^{6}$ particles and simulation times of $10^{-6} s$, atomistic models typically focus on the local molecular packing. The latter may include a basic account of supramolecular features such as a grain boundary or molecular heterojunction.

Within these limits, atomistic simulations are still rarely truly predictive as experimental input and/or physical intuition is required to set up the initial configurations. The problem of molecular self assembly in particular tends to exceed the scope of atomistic simulations due to the slow sampling of the phase space. Techniques used in crystal structure prediction therefore do not access the free energy landscape dynamically, but instead rely on MC-based techniques (notably basin hopping [59]). Even then, the computational cost of structure prediction increases significantly as the conformational properties of the molecular building blocks become more complex, to the degree that a treatment as rigid objects is no longer appropriate. Additionally, computations usually reveal several (local) minima of the energy landscape which are degenerate within the accuracy of the underlying force field. With the "true" minimum found, it still remains to be shown that the thus obtained configuration also corresponds to the minimum of the free-energy landscape relevant at finite temperature.

Within this work, we will rely on experimental input structures for crystalline systems 

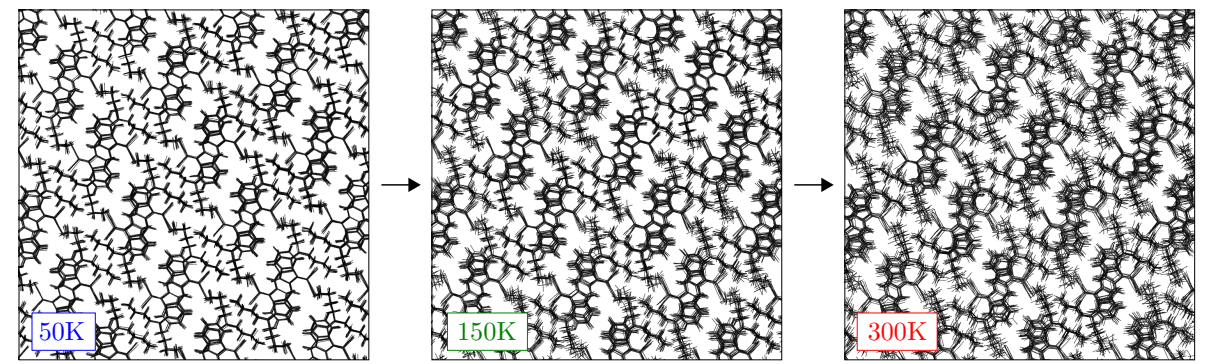

Figure 2.5. Thermalization of an EL86 crystal via stochastic dynamics. Temperature-induced conformational disorder at $T=50 \mathrm{~K}, 150 \mathrm{~K}$ and $300 \mathrm{~K}$ in an EL86 supercell assembled and optimized from its experimental X-ray structure. The simulated thermal disorder impacts electronic properties via the broadening of the density of states and thus plays a crucial role in capturing the temperature-dependence of the charge-carrier mobility.

where available. These structures, extracted, for example, from X-ray scattering spectra or electron diffraction patterns, are subsequently refined on a force-field level. This optimization has to allow for both the relaxation of nuclear coordinates, in particular the removal of extreme coordinates, and relaxation of the unit cell vectors. This can be achieved via stochastic dynamics with a strong friction coefficient in the $N P T$ ensemble at $T=0 \mathrm{~K}$. Subsequently, the system is thermalized (see Fig. 2.5) to study its stability and phase behaviour. Of particular interest is the identification of crystal polymorphs, frequently encountered in molecular materials. In some systems, including the polymeric semiconductor P3HT [60], MD simulations can access these different polymorphs, which may differ both structurally and dynamically with respect to backbone and side-chain packing - with interesting implications for charge transport [61].

Different from (poly-)crystalline materials typically used in thin-film transistors and solar cells, OLED compounds only - if at all - display rudimentary molecular ordering. These structures are then accessible through direct simulation based on a randomly generated starting configuration. This independence from experimental input is a considerable advantage for in-silico compound screening.

Beyond this simple, but predictive approach for amorphous bulk systems, in-silico deposition techniques (see Fig. 2.6) sample interfacial ordering at organic heterojunctions without the need for an initial configuration preassembled from experimental input. Still built on atomistic MD, this method may not, however, appropriately address the long relaxation times associated with the self-assembly process. Additionally, as the simulated thin-film growth velocities exceed the experimental deposition speed by many orders of magnitude, artificially induced island growth (see the height profile in Fig. 2.6b) has to be actively prevented through an accelerated surface diffusion. The latter is effectively modelled via a weighting function $w(x, y) \sim 1 / h(x, y)$ for molecule insertion that is inversely proportional to the height profile $h(x, y)$ and this way ensures homogeneous thin-film growth (Fig. 2.6c). As a considerable 

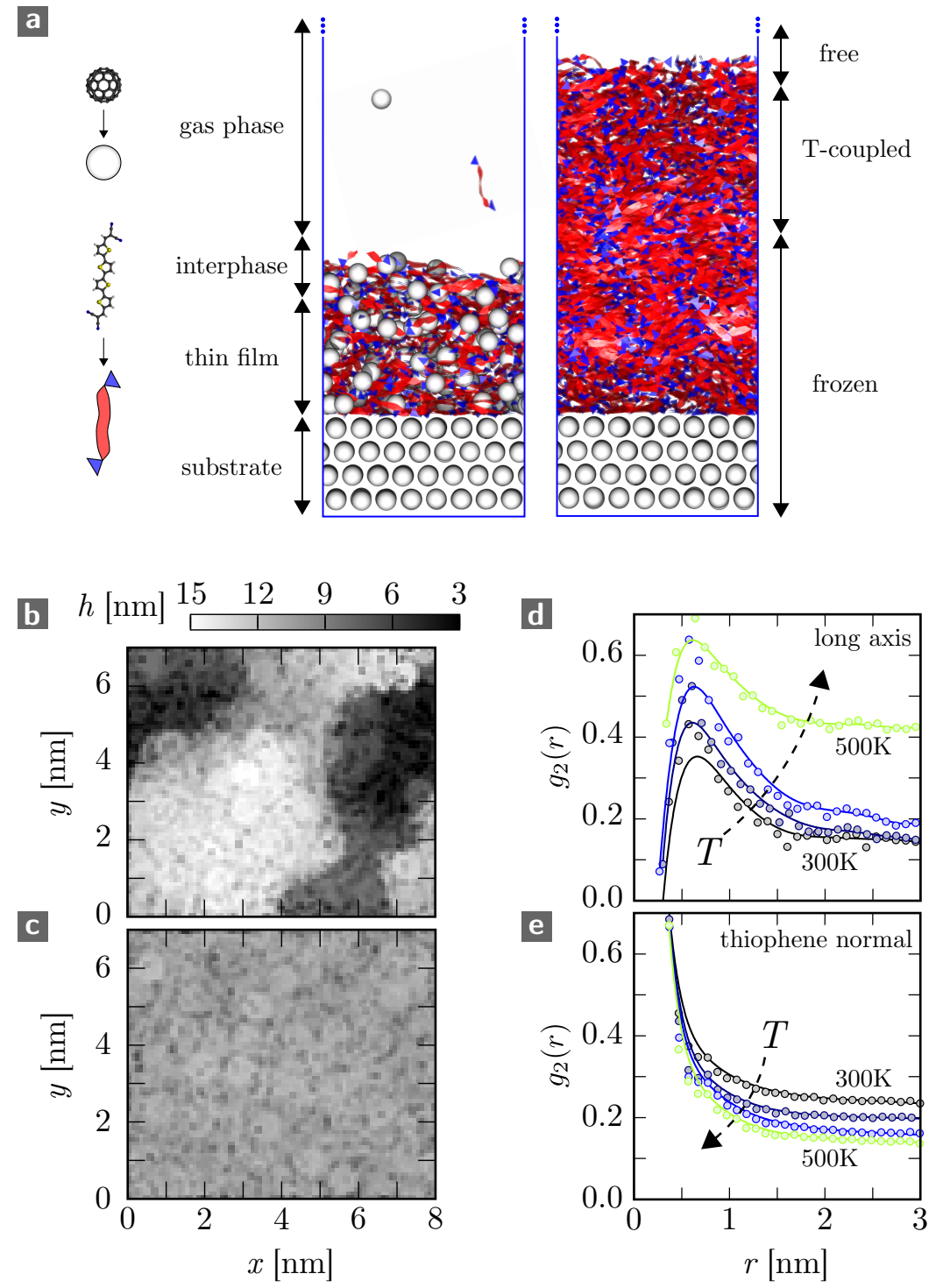

Figure 2.6. In-silico deposition approach. (a) Molecular dynamics snapshots during the coevaporation of $\mathrm{C}_{60}$ :DCV4T (right) and DCV4T evaporation (left) on a $\mathrm{C}_{60}$ substrate. In the snapshot, the two molecules are represented in a simplified manner as indicated on the very left. (b,c) Height profiles $h(x, y)$ of the coevaporated system without (b) and with (c) a linear weighting function $w(x, y) \sim 1 / h(x, y)$ for the injection of new molecules above the substrate. (d-e) Orientational correlation function for the pristine DCV4T system for the long molecular axis (d) and thiophene normals (e) at substrate temperatures of $300 \mathrm{~K}$ (black curve), $370 \mathrm{~K}$ (dark blue), $440 \mathrm{~K}$ (blue) and $500 \mathrm{~K}$ (green) after cooling and subsequent equilibration for $5 \mathrm{~ns}$ at $300 \mathrm{~K}$. 
merit of this approach, self-assembly into at least partially ordered supramolecular structures can be accessed. The characterization of these structures proceeds via suitably chosen order parameters and correlation functions that quantify dynamic and/or static properties. Fig. 2.6d-e, for example, plots the orientational correlation function

$$
g_{2}(r)=\left\langle P_{2}\left(\cos \theta_{i j}\right)\right\rangle_{r_{i j}=r},
$$

associated with the long-molecular axis (Fig. 2.6d) and thiophene normals (Fig. 2.6e) of the DCV4T molecule obtained from the analysis of a $12 \mathrm{~nm}$ thin film grown on $\mathrm{C}_{60}$. Here, $\langle\ldots\rangle$ denotes an ensemble average, $P_{2}(x)$ is the second-order Legendre polynomial, and $\theta_{i j}$ is the angle between the respective directors of molecules $i$ and $j$ at separation $r_{i j}$ in the interval $[r, r+\delta r]$. For DCV4T, the $g_{2}$ 's indicate that smectic ordering (visually perceptible in Fig.2.6a) increases, whereas $\pi$-stacking decreases with increasing substrate temperature. This liquid-crystalline ordering is simply the effect of the rod-like architecture of the molecules, under the artificially imposed constraint that the smectic layers have to line up periodically. Even though our analysis correctly indicates a face-on orientation of the DCV4T on $\mathrm{C}_{60}$ as the preferred configuration [62], dependable insights into the properties of the experimental system are beyond the scope of the approach. Nevertheless, the obtained non-equilibrium structures prove useful to understand the electronic properties of partially-ordered systems, which is indeed how we will make use of them later on in Chapters 4 and 5 .

\subsubsection{Coarse-Grained Models}

The primary challenge in the simulation of atomistic morphologies results from the slow dynamics that accompany the extremely rugged free-energy landscape to which molecular processes are subjected: These include crystallization, domain formation, the self assembly of the molecular constituents into lamellae or nanofibers, and host-guest interdiffusion.

One way to nevertheless access these processes in simulations, together with the associated length scale of several hundred nanometers, relies on coarse-graining the atomistic degrees of freedom into effective degrees of freedom representing a collection of atoms, entire monomers or even molecules [63]. Such a particle-based coarse-graining approach relies on a separation of time and length scales, where fast time scales and short length scales are implicitly absorbed in the coarse-grained particles described via effective interactions derived from atomistic reference simulations using structure- [64], force- [65] or entropy-based [66] protocols. The achieved computational speed-up is then due, first, to the reduction of explicit degrees of freedom, second, to the reduced friction in the system that follows from a smoothened free-energy surface.

An important application of such approaches targets mesophases of conjugated polymers, characterized by their slow relaxation times and long-range conformational order that ranges from lamellar-crystalline to liquid-crystalline to amorphous within the same material. Conventional coarse-graining techniques using isotropic interaction potentials for conjugated 
moieties are in this case insufficient as they disregard the anisotropic character of the $\pi$ - $\pi$ interactions that drive biaxial backbone ordering [67-71].

In the following, we will introduce a soft coarse-grained model designed to capture these interactions on a phenomenological level, while preserving enough microscopic detail to allow for reinsertion of atomistic details. The model and backmapping scheme were developed by P. Gemünden and K. Daoulas [72]: Within the model, particles represent entire repeat units of the polymer, including side chains. The local frame of these particles is given by the righthanded trihedron $\{\boldsymbol{u}, \boldsymbol{v}, \boldsymbol{w}\}$, where $\boldsymbol{u}$ points along the backbone, $\boldsymbol{w}$ along the normal of the conjugated $\pi$-plane, and $\boldsymbol{v}=\boldsymbol{w} \times \boldsymbol{u}$. Bonded interactions are derived systematically in a bottom-up manner from a Boltzmann inversion of the atomistic conformational distribution functions. The non-bonded interaction potential between two particles $i$ and $j$ is, by contrast, defined in a top-down way as

$$
V_{\mathrm{nb}}^{(i j)}=u\left(r_{i j}\right)\left[\kappa-\frac{2 \nu}{3} A_{i}: A_{j}-\frac{2 \mu}{3}\left(A_{i}: B_{j}+B_{i}: A_{j}\right)-\frac{\lambda}{2} B_{i}: B_{j}\right] .
$$

Here, the volume overlap $u\left(r_{i j}\right)$ of density clouds associated with each particle governs the interaction strength. The interaction parameter $\kappa$ controls the compressibility of the system. Orientational interactions are defined in terms of the particle tensors

$$
\begin{aligned}
& A_{i, \alpha \beta}=\frac{1}{2}\left(3 u_{\alpha} u_{\beta}-1\right), \\
& B_{i, \alpha \beta}=v_{\alpha} v_{\beta}-w_{\alpha} w_{\beta} .
\end{aligned}
$$

In the potential from Eq. 2.14, the interaction parameters $\nu$ and $\lambda$ generate a nematic and biaxial alignment, respectively, whereas $\mu$ effects an orthogonal alignment between the interaction partners, not required (as unphysical) in this context. The phenomenological potential thus mimics the $\pi$ - $\pi$ interaction between conjugated subunits. Realistic values for $\nu$ and $\lambda$ can be derived from the mechanical properties of the polymer, and are on the order of several $k_{\mathrm{B}} T[72,73]$. By tuning their relative strengths, it is furthermore possible to simulate amorphous, nematic and biaxial systems within the same framework.

As a considerable advantage of the above approach, the orientation of the particles is known throughout the entire simulation. Backmapping of the atomistic structures without loss of orientational $\pi-\pi$ ordering is hence possible. A workflow [74] for the simulation of polymeric mesophases then comprises the following steps (see Fig. 2.7): First, coarse-grained bonded potentials are derived from an atomistic reference simulation of a single chain, using Boltzmann inversion. Second, large polymeric mesophases of the respective polymer are prepared and equilibrated via Metropolis Monte-Carlo sampling with appropriately defined Monte-Carlo moves. Third, the excluded volume of the chains is recovered by applying an intermediate coarse-grained potential to the particles, derived from structure-based coarsegraining of an all-atom polymer melt. Fourth, the atomistic details are reinserted, and the obtained structure equilibrated via atomistic MD.

Well exceeding the capability of purely atomistic simulations, simulation protocols based 

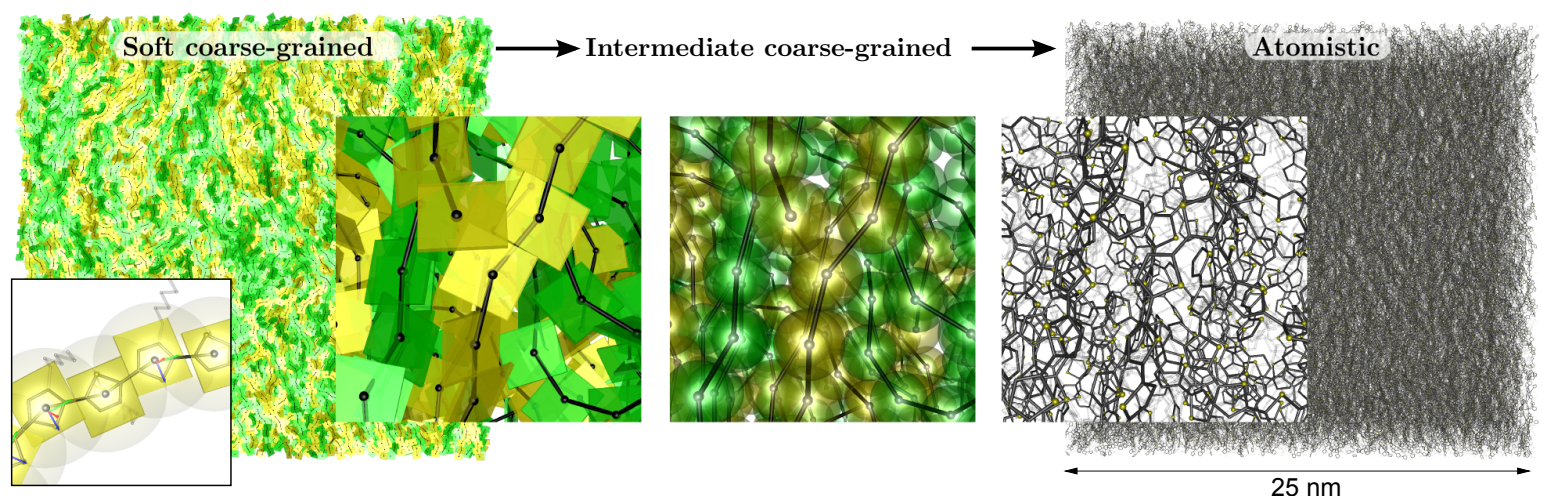

个 CG bonded potentials

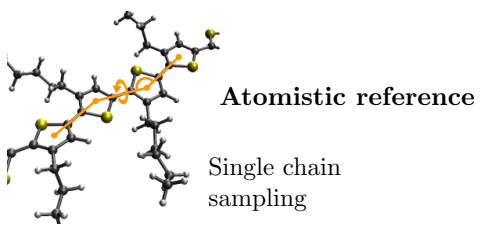

Reference RDF $\uparrow$
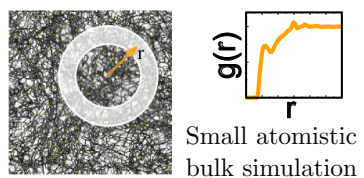

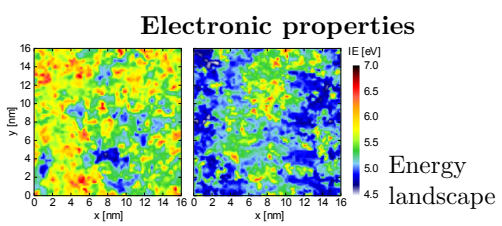

Figure 2.7. Workflow for the simulation of large models of conjugated polymers at atomistic resolution [74]. A low-resolution polymeric mesophase is simulated with a soft coarse-grained description that incorporates phenomenological non-bonded interaction potentials and bonded interactions derived from coarse-graining of an atomistic chain. Backmapping of the low-resolution model proceeds via an intermediate coarse-grained step that removes chain overlaps inherited from the soft interaction potential. Finally, atomistic details are backmapped into the coarse-grained representation, with the resulting atomistic configuration relaxed via molecular dynamics.

on soft coarse-grained models enable us to study the effect of long-range conformational disorder on electronic properties, as will be exemplified in Chapter 5 for the polymeric semiconductor P3HT.

\subsubsection{Lattice Models}

In order to study structure-property relationships in organic semiconductors, we usually require a systematic link of the theoretical description to the underlying chemical structures. At an atomistic level of resolution, this requirement is of course met, and still holds true for most coarse-grained treatments with potentials derived from an atomistic reference. Sometimes, however, more generic descriptions - here in the form of lattice models - are desirable: We will use them to distinguish between essential and non-essential features of a model, to explore finite-size effects, or to study complex morphologies (such as patterned thin films) where preassembly on an atomistic scale is artefact-prone. This broad range of applicability is due to the straightforward parametrization and efficient equilibration of such lattice models.

Within the lattice description, particles positioned at the lattice sites represent entire 
molecules, with their interactions defined in diverse ways to satisfy the purpose of the model. In the simplest case, the anisotropic sites are modelled as multipolar polarizable particles with fixed orientation - i.e., they are simply arrested in their initial, preassembled configuration. This type of lattice description will prove useful for the study of multi-layered and patterned thin films (Sec. 4.2).

Based on phenomenological Hamiltonians, disordered structures can be obtained efficiently through equilibration of the structures using Monte-Carlo techniques. To study the formation and energetics in polycrystalline systems (see Fig. 2.2c and Sec. 4.4), for example, several (approximately) periodically matched sublattices assembled from unit cells with varying orientations are superimposed and seeded independently. The individual grains then grow probabilistically around those seeds with only exclusion interactions applied: $V(i, j)=\infty$ if $r_{i j} \leq r_{c}$, else 0 , with particle indices $i, j$ and cutoff $r_{c}$.

Furthermore, Ising- and Heisenberg-type models can be used to address intrinsically disordered materials. The effects of interface roughness, for example, will be described based on a morphology simulated with a binary 3D Ising model (see Fig. 2.2b and Sec. 6.1.3), where the magnetization variable determines the chemical identity (donor $D$ or acceptor $A$ ) of the lattice particle, with phenomenological contact interactions chosen such that $\varepsilon_{A D}=\varepsilon_{D A}<$ $\varepsilon_{A A}=\varepsilon_{D D}$. The chemical composition of the system is furthermore constrained by only admitting particle swaps as Monte Carlo moves.

Finally, Heisenberg-type models access disorder-dependent properties in multipolar bulk media, notably the spatial correlation function of the site-energy landscape for charge transport, as well as associated finite-size effects. With the option to be parametrized from microscopic input, an interaction potential similar to Eq. 2.14 with an additional electrostatic contribution $\left(Q_{t}^{i} T_{t u}^{i j} Q_{u}^{j}\right.$ with interaction tensor $T_{t u}^{i j}$ and spherical-tensor multipole components $\left.Q_{t}^{i}\right)$ serves as the model basis:

$$
V_{\mathrm{nb}}^{(i j)}=\sum_{t, u} Q_{t}^{i} T_{t u}^{i j} Q_{u}^{j}-u\left(r_{i j}\right)\left[\frac{2 \nu}{3} A_{i}: A_{j}+\frac{\lambda}{2} B_{i}: B_{j}\right] .
$$

Due to the positional constraints of the lattice sites, the compressibility has been omitted from the interaction potential. Different from the soft coarse-grained case, $u\left(r_{i j}\right)$ is now a function which is constant for nearest neighbours and zero beyond. With electrostatics modelled separately, the interaction terms controlled by $\nu$ and $\lambda$ should be interpreted as only steric contributions. Already for the case of purely dipolar interactions $(\nu=\lambda=0)$ as shown in Fig. 2.8, different types of ordering can be explored, ranging from amorphous to dimerized-crystalline.

\subsection{Charge Transport Networks}

The directed graph for charge (and similarly energy) transport derives from a site-based abstraction of the atomistic morphology obtained within one of the frameworks presented 

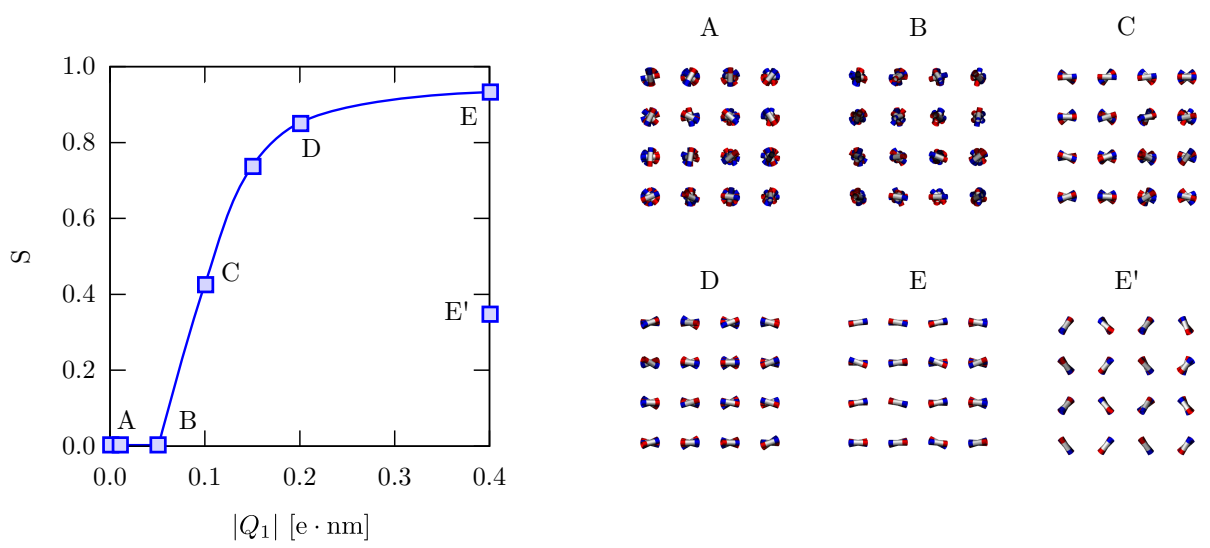

Figure 2.8. Classical Heisenberg-type lattice model of a dipolar material. Phase behaviour of the nematic order parameter $S$ with dipole strength $\left|Q_{1}\right|$ extracted from simulations of a cubic lattice with 125000 sites. In the excerpts from the snapshots (A-E'), each lattice site is represented as a small cylinder with the negative (positive) pole coloured blue (red). The ordering varies from amorphous $(\mathrm{A}, \mathrm{B})$ to dimerized (D,E). For comparison, the dipole strengths of the amorphous semiconductor Alq3 (resembling structure B) and crystalline donor-acceptor compound AAE007 (resembling structure D) amount to $\left|Q_{1}\right|=0.1$ and $\left|Q_{2}\right|=0.2$, respectively.

in Sec. 2.2. In the following, we will address the mapping procedure and in particular the parametrization of the network. As the latter has to be based on a specific transport theory, we will swiftly review the physics of charge transfer reactions in molecular systems.

\subsubsection{Charge Transfer Reactions}

For the bimolecular charge transfer reaction $A^{ \pm}+B \rightarrow A+B^{ \pm}$between molecules $A$ and $B$, a helpful model Hamiltonian reads [16, 17, 75]

$$
\hat{H}_{A B}=U_{A b}\left|\phi_{A b}\right\rangle\left\langle\phi_{A b}\left|+U_{a B}\right| \phi_{a B}\right\rangle\left\langle\phi_{a B}\left|+J_{A B}\right| \phi_{A b}\right\rangle\left\langle\phi_{a B}\left|+J_{A B}^{*}\right| \phi_{a B}\right\rangle\left\langle\phi_{A b}\right| .
$$

In this Hamiltonian, $\left|\phi_{A b}\right\rangle$ denotes the wave function of the system with molecule $A$ in its charged (upper-case $A$ ), and molecule $B$ in its neutral (lower-case $b$ ) state, and vice versa for $\left|\phi_{a B}\right\rangle . U_{A b}=U_{A b}(\boldsymbol{R})$ and $U_{a B}=U_{a B}(\boldsymbol{R})$ are the associated on-site energies, which depend on the nuclear configuration $\boldsymbol{R}$. $J_{A B}$ is the coupling that mediates the transfer process.

The above Hamiltonian is still rather general in the sense that it can describe different regimes of charge transport, as determined by the relative time scales of the electronic and nuclear degrees of freedom: Namely, $t_{J} \sim 1 / J_{A B}$ suggests a time scale for the migration of the wave function of the excess charge from $A$ to $B$, whereas $t_{\xi} \sim 1 / \hbar \omega_{\xi}$ quantifies the time scale for nuclear vibrations with frequency $\omega_{\xi}$. If $t_{J} \ll t_{\xi}$, the charge is delocalized on the time 
scale of nuclear motion and hence experiences the effective potential-energy surface (PES)

$$
U_{ \pm}(\boldsymbol{R})=\frac{1}{2}\left(U_{A b}(\boldsymbol{R})+U_{a B}(\boldsymbol{R}) \pm \sqrt{\left[U_{A b}(\boldsymbol{R})+U_{a B}(\boldsymbol{R})\right]^{2}+4\left|J_{A B}\right|^{2}}\right)
$$

obtained through diagonalization of the Hamiltonian from Eq. 2.18. In Fig. 2.9, this adiabatic PES is resolved along a promoting nuclear mode $\xi$, together with $U_{A b}$ and $U_{b A}$. The splitting of $U_{+}$and $U_{-}$at the crossing point $\xi^{*}$ amounts to $2\left|J_{A B}\right|$, and hence increases as the interaction between molecules $A$ and $B$ becomes stronger. In organic semiconductors, however, the intermolecular coupling $J_{A B}$ remains small compared to intramolecular bond vibration energies due to the weak interaction between the molecules. As a result, $t_{J} \gg t_{\xi}$, suggesting a picture where the charge "hops" from one localized state to the other, while subjected to fast vibrational motion. These localized, diabatic states differ qualitatively from adiabatic states in that their character does not depend on the nuclear coordinates: Specifically, $\left|\phi_{A b}\right\rangle$ defines the situation where the charge is localized on $A$ whatever the nuclear configuration may be. A rigorous definition for these states hence reads

$$
\left\langle\phi_{\nu}\left|\nabla_{R}\right| \phi_{\mu}\right\rangle=0
$$

where $\nabla_{R}$ denotes the gradient with respect to the nuclear coordinates. In practice, a construction of these diabatic states, to be addressed later, is challenging if approached rigorously. We proceed by calculating the rate $k_{A \rightarrow B}$ for charge hopping between the diabatic states: As the intermolecular interaction that mediates $J_{A B}$ is weak, this rate can be approximated using 1st-order perturbation theory. Fermi's Golden Rule yields

$$
k_{A \rightarrow B}=\frac{2 \pi}{\hbar} \int f(\xi)\left|J_{A B}\right|^{2} \delta\left(U_{A b}(\xi)-U_{a B}(\xi)\right) d \xi
$$

where the integral accounts for a canonical average over all configurations $\xi$ with the Boltzmann distribution function $f(\xi) \sim \exp \left[-U_{A b}(\xi) / k_{\mathrm{B}} T\right]$. Energy conservation, however, requires the transfer event to occur at the crossing point $\xi^{*}$, where $U_{A b}(\xi)=U_{a B}(\xi)$. If the promoting mode is treated classically, we obtain the Marcus rate expression [76]

$$
k_{A \rightarrow B}=\frac{2 \pi}{\hbar} \frac{\left|J_{A B}\right|^{2}}{\sqrt{4 \pi \lambda k_{\mathrm{B}} T}} \exp \left[-\frac{\left(\Delta U_{A B}-\lambda\right)^{2}}{4 \lambda k_{\mathrm{B}} T}\right] .
$$

Here, $\lambda$ denotes the classical reorganization energy that relates to the distortion of the molecular structure required to trigger the charge transfer:

$$
\lambda=\frac{1}{2} \omega_{\xi}^{2}\left(\xi_{A b}-\xi_{a B}\right)^{2}
$$

In principle, this reorganization energy also features electronic and nuclear polarization contributions from the environment that can be accounted for via an additional harmonic mode 
$\xi^{\prime}$, giving rise to the outer-sphere reorganization energy

$$
\lambda^{\text {out }}=\frac{1}{2} \omega_{\xi}^{\text {out } 2}\left(\xi_{A b}^{\prime}-\xi_{a B}^{\prime}\right)^{2}
$$

The Marcus rate from Eq. 2.22 has been applied with considerable success to charge transfer in solution and in the solid state. The classical treatment of both the intra- and intermolecular vibrational modes used in its derivation is, however, only appropriate in the high-temperature regime, where $k_{\mathrm{B}} T \gg \omega_{\xi}, \omega_{\xi^{\prime}}$. At room temperature, these conditions are barely satisfied, considering that intramolecular bond vibrations have zero-point energies on the order of $0.1 \mathrm{eV}$. This exceeds the thermal energy at room temperature by a factor of four. A more rigorous approach therefore builds on a Hamiltonian which includes a quantum-mechanical description of both the electronic and nuclear degrees of freedom:

$$
\begin{gathered}
\hat{H}_{A B}=\sum_{k, l}\left(U_{A b}^{k l}+U_{A b}^{\text {out }}\right)\left|\psi_{A b}^{k l}\right\rangle\left\langle\psi_{A b}^{k l}\left|+\sum_{k, l}\left(U_{a B}^{k l}+U_{a B}^{\text {out }}\right)\right| \psi_{a B}^{k l}\right\rangle\left\langle\psi_{a B}^{k l}\right|+ \\
\sum_{k, l, k^{\prime}, l^{\prime}} J_{A B}^{k l k^{\prime} l^{\prime}}\left|\psi_{A b}^{k l}\right\rangle\left\langle\psi_{a B}^{k^{\prime} l^{\prime}}\right|+\text { h.c. }
\end{gathered}
$$

Here, $\left|\psi_{A b}^{k l}\right\rangle=\left|\phi_{A b}\right\rangle\left|\chi_{A}^{k}\right\rangle\left|\chi_{b}^{l}\right\rangle$ is a product state with the nuclear wave functions $\chi_{A}^{k}$ and $\chi_{b}^{l}$ with vibrational quantum numbers $k$ and $l$ and frequency $\omega_{A}$ and $\omega_{b}$, respectively. $U_{A b}^{\text {out }}$ and $U_{a B}^{\text {out }}$ are the potentials that describe the coupling with a (still classical) outer-sphere mode. Assuming that the initial state from which the charge transfer reaction proceeds is the vibrational ground state with $k=l=0$, we obtain for the charge transfer rate $[50,77]$

$$
\begin{aligned}
& k_{A \rightarrow B}=\frac{2 \pi}{\hbar} \frac{\left|J_{A B}\right|^{2}}{\sqrt{4 \pi \lambda^{\text {out }} k_{\mathrm{B}} T}} \sum_{k, l}\left|\left\langle\chi_{A}^{0} \mid \chi_{a}^{k}\right\rangle\right|^{2}\left|\left\langle\chi_{b}^{0} \mid \chi_{B}^{l}\right\rangle\right|^{2} . \\
& \cdot \exp \left[-\frac{\left(\Delta U_{A B}-\hbar k \omega_{A}-\hbar l \omega_{B}-\lambda^{\text {out }}\right)^{2}}{4 \lambda^{\text {out }} k_{\mathrm{B}} T}\right] .
\end{aligned}
$$

This expression, which ultimately simplifies to the Levich-Jortner [16, 78] rate expression, demonstrates explicitly how the charge transfer between the initial and final states is mediated by the nuclear degrees of freedom. This extended theory incorporates nuclear tunnelling important at low temperatures, where the charge transfer proceeds via tunnelling through the energy barrier in the PES [79]. Still, at very low temperatures, the description of charge transfer requires a yet more realistic account of the spectral density of the phonon bath, leading to rate expressions such as those developed by Weiss and Dorsey [80, 81].

\subsubsection{Graph Generation and Parametrization}

We have rationalized in the previous section that charge transfer in molecular materials proceeds as a hopping process between diabatic, localized states. Different rate expressions that describe this process based on a classical or quantum-mechanical account of the internal 


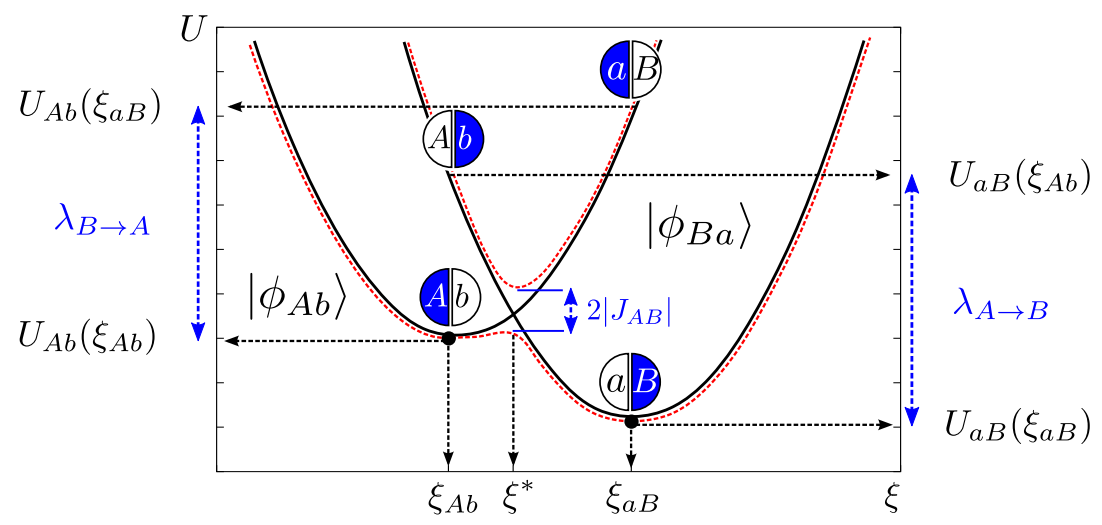

Figure 2.9. Diabatic and adiabatic potential energy surfaces. Diabatic (solid black line) and adiabatic (dashed red line) potential energy surfaces of two electronic dimer states $\left|\phi_{A b}\right\rangle$ and $\left|\phi_{a B}\right\rangle$ participating in the charge transfer reaction along the reaction coordinate (promoting, accepting mode) $\xi$. The adiabatic level splitting at the crossing point $\xi^{*}$ amounts to twice the electronic coupling $J_{A B}$. Furthermore shown are the reorganization energies $\lambda_{A \rightarrow B}, \lambda_{B \rightarrow A}$ (see Eq. 2.33).

and/or external promoting mode all share two cardinal parameters, the electronic coupling $J$ and driving force $\Delta U$. The treatment of the internal and outer-sphere reorganization differs among the theories. In the Marcus picture, however, nuclear reorganization can be captured within a single parameter $\lambda$. Among these three parameters that hence quantify the charge transfer process, $J$ and $\Delta U$ strongly depend on the conformational and packing properties of the molecular dimer and, in the case of $\Delta U$, also the molecular environment. To describe charge transport, these microscopic parameters have to be evaluated for all charge transfer pairs in an atomistically resolved morphology. These pairs and associated localization sites define the nodes and links, respectively, of a directed graph based on which charge transport in the system can be simulated.

\section{System Partitioning}

As a first step in generating the charge transport network, we have to partition the atomistic morphology onto localization sites or conjugated segments, which should be defined such that they correspond to the diabatic states in the Hamiltonian from Eq. 2.18. In small-molecular organic semiconductors, these conjugated segments correspond to entire molecules, within which charge delocalization is instantaneous. In polymeric systems, this simple assignment of localization sites may fail, as torsional defects along the conjugated backbone, variations in molecular fields, or electron-phonon interactions can enforce a stronger localization of the charge on a conjugated subunit, rather than delocalization along the entire polymer chain. In this case, one in principle has to calculate the wave function of the entire (sub)system and thus identify the conjugated segments [82, 83]. The latter can be achieved via linear scaling methods such as the tight-binding approximation of DFT (DFTB) [84, 85]. A 
computationally less demanding, but crude [86] approach is to employ a heuristic criterion for conjugation [71, 72]: Polymer chains are then partitioned onto their units of transport based on a cutoff criterion for the torsional deviation from the planar cis- and trans-conformations of two successive repeat units.

Once the conjugated segments have been identified, they are further divided into rigid fragments, i.e., structural elements that are insensitive to the influence of thermal fluctuations. As an example, conjugated units such as a thiophene or benzene ring remain planar even at elevated temperatures. From the definition of these rigid fragments, local frames - required for electrostatic parametrizations as well as orbital mapping - are obtained in a straightforward manner. Furthermore, by mapping DFT-optimized structures onto the rigid fragments, bondlength fluctuations that may introduce artefacts in the calculation of electronic couplings can be avoided.

The connectivity of the conjugated segments is described via a neighbourlist storing pairs of conjugated segments between which charge transfer may occur. This neighbourlist is constructed based on a cutoff criterion for the nearest distance of approach between any of the rigid fragments of the two sites. Finally, with the set of pairs defining the links of the directed graph, the parametrization of the network requires the evaluation of backward- and forward-rates for each of these links.

\section{Electronic Couplings}

The off-diagonal element $J_{A B}=\left\langle\phi_{A b}\left|H_{A B}\right| \phi_{a B}\right\rangle$ in the Hamiltonian from Eq. 2.18 quantifies the electronic coupling between the diabatic states $\left|\phi_{A b}\right\rangle$ and $\left|\phi_{a B}\right\rangle$, mediated by the dimer Hamiltonian $H_{A B}$. Even with the conjugated segments $A$ and $B$ identified, these diabatic states still need to be constructed. As a rigorous derivation via Eq. 2.20 is involved, approximate constructions, using techniques such as constrained DFT, are preferred [87, 88]. The cheapest solution, however, is to approximate the diabatic states via the single-site wave functions $\left|\phi_{A}\right\rangle$ and $\left|\phi_{B}\right\rangle$ of the two monomers that participate in the charge transfer. These wave functions are usually not orthogonal - a condition required for the $\left|\phi_{A b}\right\rangle$ and $\left|\phi_{a B}\right\rangle$ in Eq. 2.18. The resulting off-diagonal elements in the overlap matrix

$$
\tilde{S}_{A B}=\left(\begin{array}{cc}
1 & S_{A B} \\
S_{A B} & 1
\end{array}\right)
$$

lead to artificial contributions to the couplings. To correct for these, a transformation of the secular equation $\tilde{H}_{A B} \boldsymbol{c}=\epsilon \tilde{S}_{A B} \boldsymbol{c}$ into a standard eigenvalue equation of the form $\tilde{H}_{A B} \boldsymbol{c}=\epsilon \boldsymbol{c}$ is required [89], where $\boldsymbol{c}$ denotes the vector of expansion coefficients. Starting from $\tilde{H}_{A B}$ in the approximate, diabatic basis,

$$
\tilde{H}_{A B}=\left(\begin{array}{cc}
\bar{U}_{A b} & \bar{J}_{A B} \\
\bar{J}_{A B} & \bar{U}_{a B}
\end{array}\right) .
$$


the overlap-corrected electronic couplings to be used in the Marcus or Levich-Jortner rate expressions result after a transformation $\tilde{S}_{A B}^{-1 / 2} \tilde{H}_{A B} \tilde{S}_{A B}^{-1 / 2}$ as

$$
J_{A B}=\frac{\bar{J}_{A B}-\frac{1}{2}\left(\bar{U}_{A b}+\bar{U}_{a B}\right) S_{A B}}{1-S_{A B}^{2}} .
$$

To obtain $J_{A B}$, it is therefore sufficient to calculate $\bar{J}_{A B}=\left\langle\phi_{A}\left|H_{A B}\right| \phi_{B}\right\rangle$ using the nonorthogonal, approximate diabatic states. The Dimer-Projection (DIPRO) method [89] implemented in VOTCA computes these couplings efficiently based on the frozen-core approximation (thus assuming that only the HOMO and LUMO orbitals participate in the holeor electron-transfer, respectively) through projection of the monomer states onto the dimer states $\left\{\phi_{A B}^{i}\right\}$ :

$$
\bar{J}_{A B}=\sum_{i, j}\left\langle\phi_{A} \mid \phi_{A B}^{i}\right\rangle\left\langle\phi_{A B}^{i}\left|H_{A B}\right| \phi_{A B}^{j}\right\rangle\left\langle\phi_{A B}^{j} \mid \phi_{B}\right\rangle .
$$

We note, however, that the DIPRO formalism still requires three DFT calculations per pair, two monomer and one dimer calculation. For large systems with on the order of $10^{4}$ pairs, this implies a considerable computational expense. Though compromising on accuracy, a semi-empirical ZINDO-based approach [90, 91] parametrized for molecules with light atomic elements up to $\mathrm{Cl}$ can then be the preferred option. This approach (implemented in VOTCA::MOO) reduces the computational cost to a single monomer calculation per molecule type by constructing the diabatic states of a specific pair through appropriate rotations of the atomic orbitals onto the predefined rigid fragments.

\section{Reorganization Energies}

In a classical treatment of the intramolecular vibrations as used in the Marcus picture, the molecular reorganization is absorbed into two reorganization energies for the charging and discharging process:

$$
\begin{aligned}
& \lambda_{A \rightarrow B}=U_{a}\left(\xi_{A}\right)-U_{a}\left(\xi_{a}\right)+U_{B}\left(\xi_{b}\right)-U_{B}\left(\xi_{B}\right), \\
& \lambda_{B \rightarrow A}=U_{b}\left(\xi_{B}\right)-U_{b}\left(\xi_{b}\right)+U_{A}\left(\xi_{a}\right)-U_{A}\left(\xi_{A}\right) .
\end{aligned}
$$

The notation follows the convention from Sec. 2.3.1: $U_{a}$ and $U_{A}$, for example, denote the PES of molecule $A$ in its neutral and charged state, respectively, evaluated with the nuclear configuration $(\xi)$ of either the neutral $\left(\xi_{a}\right)$ or charged $\left(\xi_{A}\right)$ state. The reorganization energies are hence approximated via four points on the PES of each molecule, requiring a total of four SCF calculations per molecule. If the reorganization energy is assumed to not strongly fluctuate among pairs, these calculations only have to be carried out once per molecule type. In doing so, one should additionally consider that the structural reorganization in the solid state is usually sterically constrained, in particular in $\pi$-stacked arrangements. In the gasphase estimations of the reorganization energy, large conformational changes (notably ring 
rotations) should hence be prevented through appropriately chosen coordinate constraints.

\section{Driving Forces}

The site energy difference of the segments in the charge transfer pair determines the driving force $\Delta U_{A B}=U_{A}-U_{B}$ for the charge transfer reaction. For hole and electron transfer, these site energies are determined by the ionization energy and electron affinity, respectively. Due to electrostatic interactions with the molecular environment, they sensitively depend on the local and even global morphology of the material. However, as the intermolecular (as opposed to intramolecular) fields are weak, a perturbative treatment is adequate, leading to

$$
\begin{aligned}
U_{A} & =U_{A}\left(\xi_{A}\right)-U_{a}\left(\xi_{a}\right)=\left(U_{A}^{\mathrm{int}}\left(\xi_{A}\right)-U_{a}^{\mathrm{int}}\left(\xi_{a}\right)\right)+ \\
& +\left(W_{A}^{(1)}-W_{a}^{(1)}\right)+\left(W_{A}^{(2)}-W_{a}^{(2)}\right) .
\end{aligned}
$$

For $U_{B}$, an analogous expression holds. The first-order correction $\left(W_{A}^{(1)}-W_{a}^{(1)}\right)$ captures the electrostatic, the second-order correction $\left(W_{A}^{(2)}-W_{a}^{(2)}\right)$ the polarization contribution to the gas-phase site energy $U_{A}\left(\xi_{A}\right)-U_{a}\left(\xi_{a}\right)$. Finally, if the charge transfer reaction occurs under the influence of an externally applied field $\boldsymbol{F}$, an additional field term $-q \boldsymbol{F}\left(\boldsymbol{R}_{A}-\boldsymbol{R}_{B}\right)$ has to be added to the site-energy difference, where $\boldsymbol{R}_{A}, \boldsymbol{R}_{B}$ denote the positions of the molecular centers of mass.

Site-energy calculations are associated with a substantial computational expense, since the perturbative corrections in Eq. 2.34 have to be evaluated for each segment in the system. We will describe in detail in Chapter 3 how this can be achieved within a MM/MM or QM/MM long-range polarized embedding approach.

\subsection{Charge-Carrier Dynamics and Mobility}

With the transport graph fully parametrized, charge-carrier dynamics are described by a Master equation of the form

$$
\partial_{t} P_{I}(t)=\sum_{J}\left[P_{J}(t) K_{J \rightarrow I}-P_{I}(t) K_{I \rightarrow J}\right]
$$

with transition rates $K_{I \rightarrow J}, K_{J \rightarrow I}$ and time-dependent state occupation probabilities $P_{I}(t)$, $P_{J}(t)$. In the special case of a single charge carrier $(N=1)$ drift-diffusing through the system, there is a one-to-one correspondence between states and localization sites. The general expression from Eq. 2.35 then simplifies to

$$
\partial_{t} p_{A}(t)=\sum_{B}\left[p_{B}(t) k_{B \rightarrow A}-p_{A}(t) k_{A \rightarrow B}\right]
$$

with the site (rather than state) occupation probabilities $p_{A}(t), p_{B}(t)$ and charge transfer rates $k_{A \rightarrow B}, k_{B \rightarrow A}$ between the conjugated segments $A$ and $B$. For $N>1$, i.e., multiple 
charge carriers, the number of system configurations increases dramatically. For system sizes on the order of $M=10^{3}-10^{5}$ sites, direct solution of the dynamical equations is in this case prohibitive, due to the large manifold of states $\left(\sim M^{N}\right)$ as well as non-linearities that result from the interaction between the charge carriers. Kinetic Monte-Carlo (KMC) techniques [92, 93] are then the method of choice: Here, the carrier trajectories are simulated explicitly as a Markov process, with the time coordinate propagated according to the Variable-Step-Size Method (VSSM). The latter is mandatory as charge transfer rates may span many orders of magnitude, leading to site escape times anywhere from $10^{-15}$ to $10^{-9} \mathrm{~s}$.

In the KMC approach, charge transfer rates can be updated on the fly to take, for example, the Coulomb interaction between charge carriers into account. Furthermore, the formalism easily accommodates multiple types of electronic states (excitons, charges and charge-transfer states) including their interactions and mutual conversions - provided, of course, the transport graph has been parametrized accordingly.

Within the kinetic description, physical observables result as averages over the explicit trajectories. One such observable is the charge-carrier mobility tensor $\tilde{\mu}$, which linearly relates the charge-carrier velocity $\boldsymbol{v}$ to the external field $\boldsymbol{F}$ via $\boldsymbol{v}=\tilde{\mu} \boldsymbol{F}$. It can be calculated directly from the site occupation fractions:

$$
\tilde{\mu}_{\alpha \beta}=\frac{1}{N|\boldsymbol{F}|^{2}} \sum_{A, B} k_{A \rightarrow B} p_{A}\left(1-p_{B}\right)\left(R_{A, \alpha}-R_{B, \alpha}\right) F_{\beta} .
$$

Here, the exclusion term $\left(1-p_{B}\right)$ reflects the constraint that sites must only be singly occupied. Alternatively, the mobility is obtained as an end-point average over individual trajectories $\Delta R_{i}$ after time $T$ of charge carrier $i \in\{1, \ldots, N\}$,

$$
\tilde{\mu}_{\alpha \beta}=\left\langle\frac{1}{N} \sum_{i=1}^{N} \frac{\Delta R_{\alpha, i} F_{\beta}}{T|\boldsymbol{F}|^{2}}\right\rangle .
$$

Here, averaging $\langle\ldots\rangle$ occurs over different KMC runs and system configurations (e.g., MD snapshots). The macroscopic charge-carrier mobility is thus computed as a function of temperature, charge-carrier density and field strength, where, however, finite-size effects inherent to microscopic simulations may have to be accounted for, especially at low temperatures, small charge-carrier densities, and/or large energetic disorder [94, 95]. The results from such simulations can finally be used to parametrize continuous drift-diffusion models [96], and in this way calculate I-V curves of simple electronic devices. 


\section{Chapter 3}

\section{Long-RAnge Polarized EMBEDding of ElECTROnic Excitations}

In this chapter, we develop an embedding technique for the perturbative classical description of molecular excitations in organic solids - notably charge, charge transfer, and excitonic states. As an essential feature of the technique, it rigorously accounts for the long-ranged interaction of charged molecular excitations with a net-quadrupolar environment. The conditionality of the underlying interaction sum is removed through appropriately chosen shape corrections that impose bulk or thin-film conditions. The aperiodic excitation and accompanying polarization cloud are embedded in a periodic molecular background that gives rise to mesoscale fields acting upon the polarization cloud. The scheme is designed to quantitatively describe the density of states using large atomistic models. To tackle the required system sizes, it makes use of a classical expansion of the molecular field and field response in terms of distributed multipoles and polarizabilities.

\subsection{The Mesoscopic Interaction Range}

Knowledge of the energy landscape for charge and energy transport is key to the understanding and optimization of organic optoelectronic devices, such as solar cells and light-emitting diodes. As detailed in Chapter 1, their functionality relies on the dynamics of three types of microscopic states: charge carriers (electrons and holes), Frenkel excitons, and chargetransfer states - the latter being the intermediate state in the generation of free charges from or recombination to a Frenkel exciton. Featuring in level profiles across organic heterojunctions [97-99], grain boundaries, or level overlap in binary mixtures [100], the densities of states of these three microscopic species determine the macroscopic steady-state properties of the materials and devices. Encoded into the rate expressions for charge and energy transfer (Sec. 2.3.1), the sensitive relationship between dynamics and energetics controls, for example, charge-carrier mobilities, short-circuit currents, open-circuit voltages or material wearout. It is therefore a key challenge in computational materials science to predict these 
densities of states from first principles, and ultimately to establish their connection to device characteristics [18, 28, 50, 97-99].

In Sec. 2.2, we have meanwhile argued that quantum-mechanical models and classical force fields already provide accurate descriptions of single-molecule properties [101-103], but seldom provide an adequate account of supramolecular organization in ordered or partially ordered materials. The density of states, however, is expected to pick up on, for example, the interfacial structure across heterojunctions on smaller length scales, long-wavelength fluctuations of conformational order in polymeric systems, or coexistence of ordered and disordered mesophases on larger length scales. As a quantitative treatment usually requires an atomistic resolution, system sizes addressed in today's simulations may in particular prove insufficient to sample the tail of the densities of states, which determines the charge-carrier mobility. Possible solutions to some of these finite-size effects include statistical extrapolation techniques [28] or coarse-grained descriptions with reinsertion of atomistic details [72].

Still, the need to go beyond the local molecular ordering associated with a length scale of some nanometers is not only due to sampling requirements: It is additionally motivated by the fact that organic semiconductors are made of quadrupolar building blocks [104, 105], which give rise to an uncompensated quadrupole moment that in ordered systems extends to the mesoscale and beyond. For computations, this complicates matters, as the interaction of a charge with a 3D-periodic net-quadrupolar environment is only conditionally convergent [106]. For 2D-periodic systems used to describe thin films, by contrast, the convergence of the interaction sum is absolute, but very slow. For illustration, Fig. 3.1a-c provides a summary of its convergence behaviour in different 3D- and 2D-periodic systems, indicating how the electrostatic contribution $W^{(1)}$ to the energies of different electronic states changes with the size of the molecular cluster $d_{c}$ on which computations are performed. For charges embedded in a $20 \mathrm{~nm}$-thin film (Fig. 3.1b), convergence is not even achieved for a cluster size larger than $100 \mathrm{~nm}$, whereas a bulk description (Fig. 3.1a) may give the false impression that energetics are converged as of a cluster size of $8 \mathrm{~nm}$ - when in fact the associated contribution will only apply to a spherical shell-by-shell growth, and differ dramatically for other cluster shapes (cylindrical, cuboidal, etc.). In a similar fashion, the electrostatic contribution to the energy of charge transfer (CT) states (Fig. 3.1c) appears to converge faster, when in fact the individual contributions of the CT-hole and CT-electron exhibit the same slow convergence that typifies the thin-film scenario from Fig. 3.1b.

As long-range, crystalline and liquid-crystalline (and hence at least uniaxial) ordering prevails in most organic materials and devices, even including bulk heterojunctions [62, 107], an account of the ensuing long-range effects on the energy landscape is indispensable. In the following, we will detail the theoretical foundation of the embedding technique developed as part of this thesis and implemented in the VOTCA suite [50]. Its application to both atomistic and lattice models of organic bilayers and trilayers, ordered and disordered systems will follow in Chapters 4 to 5, where we will investigate structure-energy relationships through the comparison of different molecular architectures, packing modes and ordering, indicating how a long-range treatment not only proves crucial in quantifying long-range effects in mesoscopi- 


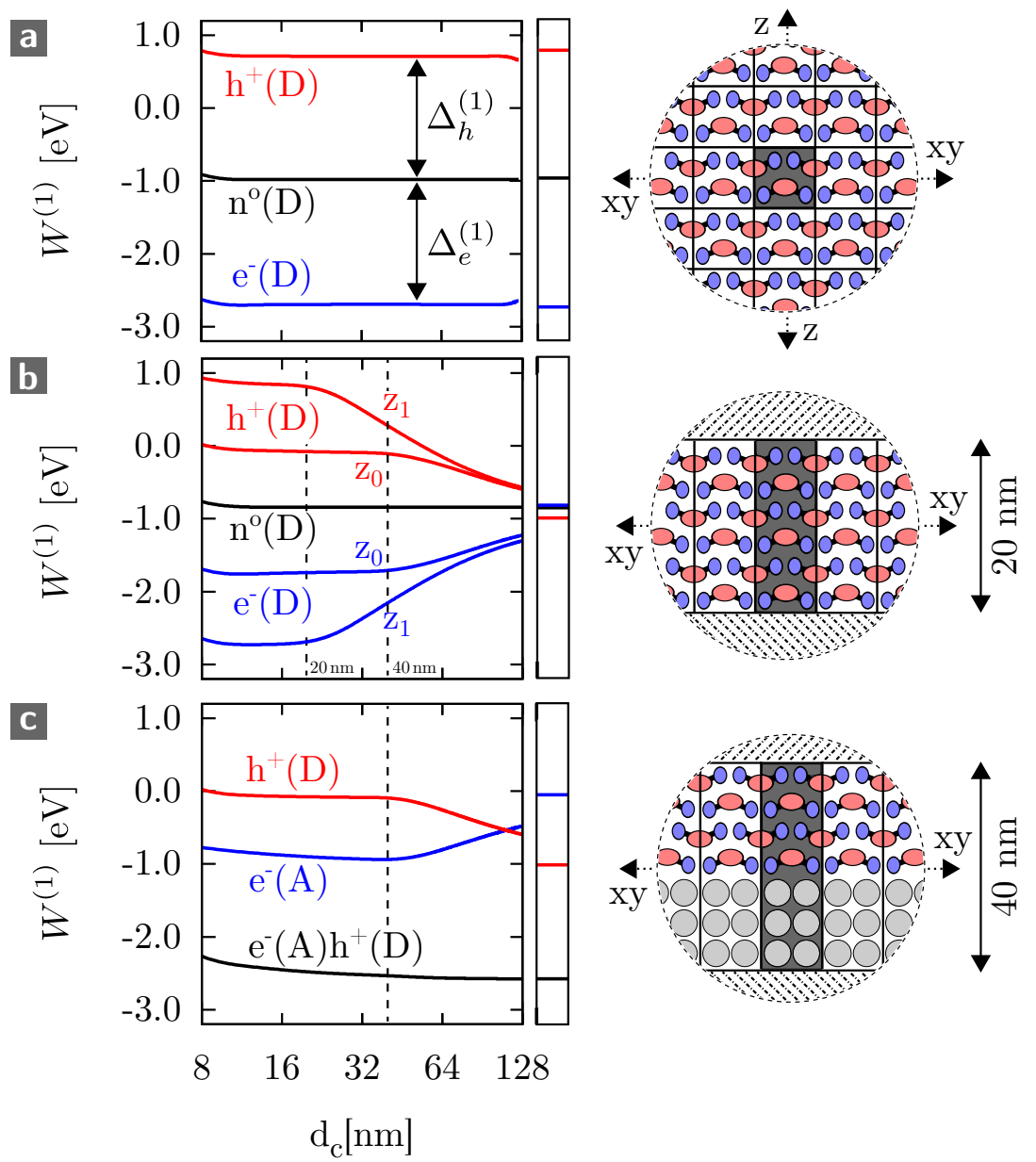

Figure 3.1. Energetics on a mesoscale. Variation of electrostatic configuration energies $W^{(1)}$ with the cluster size $d_{c}$ for a bulk (a) and thin-film (b,c) setup. In (a) and (b), black, red, and blue lines denote trends for neutral $\left(n^{o}\right)$, hole $\left(h^{+}\right)$and electron $\left(e^{-}\right)$states, localized on a donor (D, here: D5M) or acceptor (A, here: $\mathrm{C}_{60}$ ) molecule, respectively. In (b), $z_{0}$ and $z_{1}$ refer to a molecular ion positioned at the centre and surface of the film, respectively. In (c), the black curve denotes the convergence trend for an interfacial charge transfer state, broken down into the contributions of the CT hole (red curve) and CT electron (blue curve) in the same plot. Configuration energies $W^{(1)}$ for the infinite systems are shown on the very right of each plot, where for the bulk setup a macroscopically spherical shape has been chosen to remove the conditional convergence for the 3D-periodic case, which corresponds to the spherical shell-by-shell growth of the molecular cluster of size $d_{c}$. The schematics on the right-hand side indicate the system under study including cartoons of the two molecular species (D5M and $\mathrm{C}_{60}$ ), with a system repeat unit coloured in black, and periodicity indicated by dashed arrows. 
cally ordered systems, but also in correcting for finite-size-induced artefacts in mesoscopically amorphous systems.

\subsection{The Molecular Field and Field Response}

In molecular solids, the energy of a particular molecular state derives from the interaction of the excited molecular cluster with its surrounding [61]. In order to compute this energy, we first require models to describe the molecular field and field response. In this section, we will detail how the computational complexity associated with this interaction can be tackled, leading to an expansion of molecular properties in terms of distributed atomic properties [108, 109].

As the primary contribution to the interaction is of an electrostatic classical (rather than exchange) character, the associated additional term in the Hamiltonian of a molecule reads

$$
W^{(1)}=\int d^{3} x \rho(\boldsymbol{x}) \varphi^{\operatorname{ext}}(\boldsymbol{x}) .
$$

This is the classical configuration energy of a charge density $\rho(\boldsymbol{x})$ in an external potential $\varphi^{\text {ext }}(\boldsymbol{x})$. This charge density follows immediately from the molecular wave function $\Psi(r, \sigma ; R)$ with collective electronic, spin and nuclear coordinates $r, \sigma$ and $R$, respectively:

$$
\begin{aligned}
\rho(\boldsymbol{x}) & =-e \sum_{j=1}^{N_{e}} \sum_{\sigma} \int d r d R \delta\left(\boldsymbol{r}_{j}-\boldsymbol{x}\right)|\Psi(r, \sigma ; R)|^{2}, \\
& +\sum_{J=1}^{N_{n}} e Z_{J} \sum_{\sigma} \int d r d R \delta\left(\boldsymbol{R}_{\boldsymbol{J}}-\boldsymbol{x}\right)|\Psi(r, \sigma ; R)|^{2} .
\end{aligned}
$$

This charge density in turn generates an electrostatic potential $\varphi(\boldsymbol{x})$ acting on the molecular surrounding via

$$
\varphi(\boldsymbol{x})=\frac{1}{4 \pi \varepsilon_{0}} \int d^{3} x^{\prime} \frac{\rho\left(\boldsymbol{x}^{\prime}\right)}{\left|\boldsymbol{R}-\boldsymbol{x}^{\prime}\right|} .
$$

The external potential in Eq. 3.1 can be thought of as having the same molecular origin. Since this intermolecular potential is typically weak compared to the intramolecular potential, it can be treated perturbatively. The first- and second-order perturbative corrections are formally given by [110]

$$
\begin{aligned}
& W^{(1)}=\langle 0|\hat{W}| 0\rangle \\
& W^{(2)}=-\sum_{n \neq 0} \frac{|\langle 0|\hat{W}| n\rangle|^{2}}{E_{n}-E_{0}} .
\end{aligned}
$$

We will refer to the first-order term as the electrostatic, to the second-order term as the 
polarization contribution. Unfortunately, $W^{(1)}$ and $W^{(2)}$ as written down above in terms of integrals over the continuous molecular wave function are not computationally accessible for the large systems of interest here. In the following, we will therefore describe approaches how these expressions can be reformulated in terms of distributed molecular/atomic multipoles and polarizabilities that allow us to compute these perturbative corrections efficiently in a classical expansion.

\subsubsection{Distributed Multipoles}

For a system composed of only two molecules $A$ and $B$ located at positions $\boldsymbol{X}$ and $\boldsymbol{Y}$, respectively, the first-order contribution $W^{(1)}$ is given by the standard Poisson integral

$$
W_{A B}^{(1)}=\frac{1}{4 \pi \varepsilon_{0}} \iint d^{3} x d^{3} y \frac{\rho_{A}(\boldsymbol{x}) \rho_{B}(\boldsymbol{y})}{|\boldsymbol{Y}+\boldsymbol{y}-\boldsymbol{X}-\boldsymbol{x}|} .
$$

We will now transform this integral such that the integration over $\boldsymbol{x}$ and $\boldsymbol{y}$ can be performed independently of each other. To this end, we consider the expansion of the inverse distance $|\boldsymbol{Y}+\boldsymbol{y}-\boldsymbol{X}-\boldsymbol{x}|^{-1}$ in terms of regular $\left(R_{l m}\right)$ and irregular $\left(I_{l m}\right)$ spherical harmonics:

$$
\frac{1}{|\boldsymbol{Y}+\boldsymbol{y}-\boldsymbol{X}-\boldsymbol{x}|}=\sum_{l=0}^{\infty} \sum_{m=-l}^{m=+l}(-1)^{m} R_{l,-m}(\boldsymbol{x}-\boldsymbol{y}) I_{l, m}(\boldsymbol{Y}-\boldsymbol{X})
$$

This expression is valid only if $|\boldsymbol{x}-\boldsymbol{y}|<|\boldsymbol{X}-\boldsymbol{Y}|$. For the integral $W_{A B}^{(1)}$, we hence require that the charge densities do not interpenetrate in such a way that the above inequality holds wherever $\rho_{A}(\boldsymbol{x}), \rho_{B}(\boldsymbol{y}) \neq 0$. To further disentangle $\boldsymbol{x}$ and $\boldsymbol{y}$, we use the spherical harmonic addition theorem

$$
\begin{gathered}
R_{l m}(\boldsymbol{x}-\boldsymbol{y})=\sum_{l_{1} l_{2}} \sum_{m_{1} m_{2}} \delta_{l_{1}+l_{2}, l}(-1)^{l+m} \sqrt{\frac{(2 l+1) !}{\left(2 l_{1}\right) !\left(2 l_{2}\right) !}} \\
\cdot R_{l_{1} m_{1}}(\boldsymbol{x}) R_{l_{2} m_{2}}(-\boldsymbol{b})\left(\begin{array}{ccc}
l_{1} & l_{2} & l \\
m_{1} & m_{2} & -m
\end{array}\right) .
\end{gathered}
$$

After some transformations [110], the expansion of the interaction distance in the above fashion yields a simple expression for the interaction between the two molecules in terms of molecule-centred multipole moments $Q_{t}^{A}$ and $Q_{u}^{B}$.

$$
W_{A B}^{(1)}=Q_{t}^{A} T_{t u}^{A B} Q_{u}^{B}
$$

Here, the subscripts $t$ and $u$ (for which the Einstein sum convention is in place) denote the components of the real regular spherical harmonics $R_{l \mu}$ with $l \mu=00,10,1 c, 1 c, 20, \ldots$ The 
multipole moments are obtained as

$$
Q_{t}^{A} \leftrightarrow Q_{l \mu}^{A}=\int d^{3} x \rho_{A}(\boldsymbol{x}) R_{l \mu}(\boldsymbol{x})
$$

These real multipole moments are related to their complex counterparts via the linear combination

$$
\left(\begin{array}{l}
Q_{l+m} \\
Q_{l-m}
\end{array}\right)=\frac{1}{\sqrt{2}}\left(\begin{array}{cc}
(-1)^{m} & i(-1)^{m} \\
1 & -i
\end{array}\right)\left(\begin{array}{l}
Q_{l m c} \\
Q_{l m s}
\end{array}\right) .
$$

The $T_{t u}^{A B}$ are the tensors that mediate the interaction between the multipolar charge densities, here expressed in terms of the so-called $S$-functions [111] that absorb the orientation dependence:

$$
T_{t u}^{A B} \leftrightarrow T_{l_{1} \mu_{1} l_{2} \mu_{2}}^{A B}=\frac{1}{4 \pi \varepsilon_{0}}\left(\begin{array}{c}
l_{1}+l_{2} \\
l_{1}
\end{array}\right) S_{l_{1} l_{2} l_{1}+l_{2}}^{\mu_{1} \mu_{2}}|\boldsymbol{X}-\boldsymbol{Y}|^{-l_{1}-l_{2}-1} .
$$

Note that, as Eq. 3.9 is still based on molecule-centred moments, it cannot yet be applied to molecular solids with their dense packing and pronounced anisotropy of the molecular building blocks. The expression breaks down if the molecular charge densities interpenetrate in a radial sense, necessitating a distributed description built on atomic (or otherwise chosen) expansion sites [108]. The latter is obtained via a multipole expansion of the atomic-orbitalpair contributions to the density matrix, rather than of the molecular charge density as a whole:

$$
\rho(\boldsymbol{x})=\sum_{\alpha, \beta} \rho_{\alpha \beta} g_{\alpha}\left(\boldsymbol{x}-\boldsymbol{s}_{\alpha}\right) g_{\beta}\left(\boldsymbol{x}-\boldsymbol{s}_{\beta}\right)
$$

Here, $g_{\alpha}=R_{L K}\left(\boldsymbol{x}-\boldsymbol{s}_{\alpha}\right) \exp \left[-\zeta\left(\boldsymbol{x}-\boldsymbol{s}_{\alpha}\right)^{2}\right]$ denotes a Gaussian atomic basis function as typically used in quantum-chemical computations. The multipole component associated with the pair density of these Gaussian basis functions reads

$$
Q_{l k}[\boldsymbol{P}]=-\int R_{l k}(\boldsymbol{x}-\boldsymbol{P}) \rho_{\alpha \beta} g_{\alpha} g_{\beta} d^{3} x
$$

The thus computed multipole component $Q_{l k}$ is still referred to the overlap centre $\boldsymbol{P}$ of the two Gaussians. It can be shifted to an expansion site at position $\boldsymbol{S}$ according to

$$
Q_{n m}[\boldsymbol{S}]=\sum_{l=0}^{L} \sum_{k=-l}^{l}\left[\left(\begin{array}{c}
n+m \\
l+k
\end{array}\right)\left(\begin{array}{c}
n-m \\
l-k
\end{array}\right)\right]^{1 / 2} R_{n-l, m-k}(\boldsymbol{S}-\boldsymbol{P}) \cdot Q_{l k}[\boldsymbol{P}] .
$$

For sharply peaked pairs of Gaussian functions, this allocation can be performed on a nearestsite basis. For diffuse functions, however, a smooth weighting function in combination with grid-based integration techniques may be necessary to reduce the basis-set dependence [101]. 
Finally, we note that a set of distributed multipoles can also be obtained by fitting multipoles to atomic positions in such a way that they reproduce the electrostatic potential generated by the molecular charge density outside the molecule [112-114]. The CHELPG[114] (CHarges from ELectrostatic Potentials, Grid-based) algorithm, for example, derives these charges from a least-squares fit that operates on the objective function

$$
z\left(\left\{q_{i}\right\}\right)=\sum_{k=1}^{M}\left(\phi\left(\boldsymbol{r}_{k}\right)-\sum_{i=1}^{N} \frac{1}{4 \pi \varepsilon_{0}} \frac{q_{i}}{\left|\boldsymbol{r}_{i}-\boldsymbol{r}_{k}\right|}\right)+\lambda\left(q_{\mathrm{mol}}-\sum_{i=1}^{N} q_{i}\right),
$$

with $M$ grid points, $N$ atomic sites, the set of atomic partial charges $\left\{q_{i}\right\}$ and the potential $\varphi$ from Eq. 3.3. $\lambda$ is a Lagrange multiplier that constrains the net charge to the desired value $q_{\mathrm{mol}} \cdot$

\subsubsection{Distributed Polarizabilities}

Just as distributed multipoles simplify the computation of the first-order, electrostatic contribution, distributed polarizabilities achieve the same for the second-order, polarization contribution. To get there, we write the perturbing field in terms of multipolar contributions as $W=Q_{t}^{a} \varphi_{t}^{a}$, with atomic position superscript $a$ and rank- $t$ potential operator $\phi_{t}^{a}$. The second-order correction

$$
W^{(2)}=-\sum_{n \neq 0} \frac{\left|\left\langle 0\left|\hat{Q}_{t}^{a} \phi_{t}^{a}\right| n\right\rangle\right|^{2}}{W_{n}-W_{0}} .
$$

can then be conceptually simplified by defining correlated point-point polarizabilities $\alpha_{t t^{\prime}}^{a a^{\prime}}$ as

$$
\alpha_{t t^{\prime}}^{a a^{\prime}}=\sum_{n \neq 0} \frac{\left\langle 0\left|\hat{Q}_{t}^{a}\right| n\right\rangle\left\langle n\left|\hat{Q}_{t^{\prime}}^{a^{\prime}}\right| 0\right\rangle}{W_{n}-W_{0}}+\text { h.c. }
$$

These polarizabilities describe the distortion of the molecular charge density under the influence of the applied field $Q_{t}^{a} \varphi_{t}^{a}$. This distortion generates induced moments $\Delta Q_{t}^{a}=-\alpha_{t u}^{a b} \varphi_{u}^{b}$, obtained from the derivative of the polarization energy with respect to the field component $\varphi_{t}^{a}$. For the polarization energy, one obtains the bilinear form

$$
W^{(2)}=-\frac{1}{2} \varphi_{t}^{a} \alpha_{t t^{\prime}}^{a a^{\prime}} \varphi_{t^{\prime}}^{a^{\prime}}
$$

In practice, correlated polarizabilities of this type are not straightforward to calculate and expensive to use [109]. These polarizabilities can, however, be localized in a systematic manner $\left(\alpha_{t t^{\prime}}^{d d^{\prime}} \rightarrow \alpha_{t}^{d} \neq 0\right.$ if $\left.t \in\{1 x, 1 y, 1 z\}\right)$, leading to a local-dipole representation of the field response. Instead of a systematic derivation of these local point polarizabilities, starting from the more complicated correlated quantities, we will here follow the Thole model [103, 115], which assigns generic polarizabilities to atoms based on their element type. These polarizabilities are subsequently only correlated in that they influence each other via the 
fields they generate. This induced-induced interaction may, however, lead to a polarization catastrophe at short interseparations, as an artefact of the sharp (rather than quantummechanically diffuse) point-dipole description [116]. This overpolarization can be avoided by including a smearing function $\rho_{s}(\boldsymbol{u})$ that damps the dipole-dipole interaction at small scales, starting from the potential of a smeared point charge:

$$
\phi(u)=-\frac{1}{4 \pi \varepsilon_{0}} \int_{0}^{u} 4 \pi u^{\prime 2} \rho_{s}\left(u^{\prime}\right) d u^{\prime} .
$$

Here, $\boldsymbol{u}=\boldsymbol{u}(\boldsymbol{R})$ is an effective interaction distance to be specified later. The dipole-dipole interaction tensor $T_{i j}$ (here in Cartesian representation) can be related to the smearing density by first expressing it in terms of the effective interaction range,

$$
T_{i j \ldots}(\boldsymbol{R})=f\left(\alpha^{a} \alpha^{b}\right) t_{i j \ldots}\left(\boldsymbol{u}\left(\boldsymbol{R}, \alpha^{a} \alpha^{b}\right)\right),
$$

and subsequently demanding that $t_{i j \ldots}$ follows from the respective derivative of the modified interaction potential in Eq. 3.20:

$$
t_{i j \ldots}(\boldsymbol{u})=-\partial_{u_{i}} \partial_{u_{j}} \ldots \phi(\boldsymbol{u})
$$

Even though a variety of different smearing functions $\rho_{s}(u)$ have been tested, we here limit ourselves to

$$
\rho_{s}(u)=\frac{3 a}{4 \pi} \exp \left(-a u^{3}\right)
$$

as used in the AMOEBA force field [117]. The effective interaction distance depends on the isotropic polarizabilities $\alpha^{a}$ and $\alpha^{b}$ of the interaction partners:

$$
\boldsymbol{u}(\boldsymbol{R}) \rightarrow \boldsymbol{u}^{a b}(\boldsymbol{R})=\boldsymbol{R} /\left(\alpha^{a} \alpha^{b}\right)^{1 / 6}
$$

A set of generic element-based polarizabilities $\left(\alpha_{C}=1.334, \alpha_{H}=0.496, \alpha_{N}=1.073, \alpha_{O}=\right.$ $0.873, \alpha_{S}=2.926 \AA^{3}$ ) is sufficient to quantitatively reproduce the molecular polarizability of a variety of organic compounds [115]. These polarizabilities are, however, too small to describe the field response of $\pi$-conjugated molecules with their more mobile electron cloud. A scaling approach that reproduces the volume of the polarizability ellipsoid computed via DFT generates an optimized set of atomic polarizabilities, where non-conjugated moieties such as aliphatic side chains are exempt from the scaling. The resulting scaling factors for the original Thole polarizabilities (see table in Fig. 3.2b) lie between 1.2 and 2.0. Fig. 3.2a shows how the dielectric constant $\varepsilon_{\mathrm{cm}}$ obtained from the Clausius-Mossotti relationship, as well as the mean-field stabilization $\Delta^{\left(\varepsilon_{\mathrm{cm}}\right)}$ of a charge embedded in a cavity of radius $a$,

$$
\Delta^{\left(\varepsilon_{\mathrm{cm}}\right)}=-\frac{1}{8 \pi \varepsilon_{0}} \frac{\varepsilon_{\mathrm{cm}}-1}{\varepsilon_{\mathrm{cm}}} \frac{q^{2}}{a},
$$




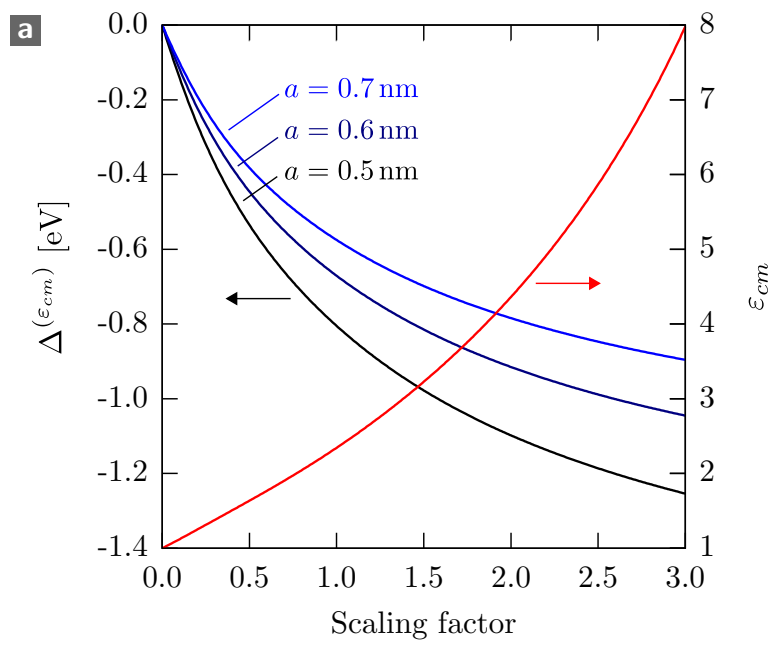

\begin{tabular}{c|ccc} 
b & \multicolumn{3}{|c}{ Scaling factor } \\
& $n$ & $e$ & $h$ \\
\hline Alq3 & 1.26 & 3.94 & 3.42 \\
DPBIC & 1.21 & 3.65 & 1.55 \\
$6 \mathrm{~T}$ & 1.65 & 2.68 & 2.31 \\
L20 & 1.65 & 2.68 & 2.31 \\
PEN & 1.67 & 2.19 & 1.80 \\
EL86 & 1.67 & 1.95 & 1.64 \\
ZnPc & 1.96 & 2.13 & 1.85 \\
D5M & 2.02 & 3.14 & 2.44
\end{tabular}

Figure 3.2. Atomic polarizability scaling factor for $\pi$-conjugated molecules. (a) Mean-field polarization stabilization $\Delta^{\varepsilon_{\mathrm{cm}}}$ (Eq. 3.25) for different cavity radii $a$ and ClausiusMossotti dielectric constant $\varepsilon_{\mathrm{cm}}$ in dependence on the scaling factor applied to atomic polarizabilities as derived by van Duijnen et al. [115] $\left(\alpha_{C}=1.334, \alpha_{H}=0.496, \alpha_{N}=1.073\right.$, $\alpha_{O}=0.873, \alpha_{S}=2.926 \AA^{3}$ ). The compound used for this example is the organic semiconductor D5M. (b) Scaling factors obtained for eight example compounds (see Table 1.1 for chemical structures) in their neutral $(n)$, electron $(e)$ and hole $(h)$ charge states.

depend on the scaling factor in the case of the organic semiconductor D5M (see Table 1.1 for the chemical structure). It can be seen that even for a cavity size of merely $0.5 \mathrm{~nm}$, the induction contribution only experiences a small decrease of ca. $0.1 \mathrm{eV}$ as the dielectric constant increases from 3 (scaling factor 1.5) to 4 (scaling factor 1.9). This indicates that the scaling procedure should be sufficiently robust to accurately capture the mean-field contribution to site energies, without losing the geometric sensitivity inherited from the distributed polarizability approach.

\subsection{Long-Range Polarized Embedding}

Having developed the necessary tools to describe the molecular field and field response, we will continue with the long-range polarized embedding approach tailored to describe molecular excitations in the solid state. As detailed in the introduction to this chapter, an approach of this kind has to be able to address large system sizes at atomistic resolution - two criteria, which we will swiftly recapitulate:

First, large system sizes are required in order to adequately sample the tail of the densities of states that results from thermal, conformational and positional disorder. In addition to this sampling requirement, typical thin-film thicknesses employed in devices are on the order of tens of nanometers. The out-of-plane dimensions should if possible be simulated to scale, whereas the large in-plane extension, being on the order of micrometers, has to be modelled 
indirectly through appropriately chosen periodic boundary conditions.

Second, an atomistic resolution is typically necessary to obtain quantitative results. Due to computational limitations, the need for this high resolution conflicts with the need for large system sizes, such that appropriately parametrized classical models with an effective treatment of electronic degrees of freedom are preferred over more expensive quantum-classical or pure quantum models.

\subsubsection{Polarization Energy and Work}

In the organic solid state, key corrections to energy levels result from the electrostatic and polarization interaction with the environment. Both these interactions lead to energy contributions on the order of $1 \mathrm{eV}$ and are hence indispensable in a physical description. They can, however, be treated perturbatively, as shown in Sec. 3.2. The electrostatic and polarization contribution then correspond to the first- and second-order corrections $W_{s}^{(1)}$ and $W_{s}^{(2)}$, respectively, where the subscript $s$ denotes the state of the system. With $s=n$ referencing the neutral ground state, the energy corrections $\Delta_{s}$ to site energies read

$$
\begin{aligned}
\Delta_{s}^{(i)} & \equiv W_{s}^{(i)}-W_{n}^{(i)}, \\
\Delta_{s}^{(1,2)} & =\Delta_{s}^{(1)}+\Delta_{s}^{(2)}, \\
W_{s}^{(1,2)} & =W_{s}^{(1)}+W_{s}^{(2)} .
\end{aligned}
$$

These $\Delta$ 's are the perturbative corrections to ionization energies (IEs) $(s=h)$ and electron affinities (EAs) $(s=e)$ in the solid state. The total IE of a molecule, for example, reads $\mathrm{IE}=\mathrm{IE}_{0}+\Delta_{h}^{(1,2)}$, where $\mathrm{IE}_{0}$ denotes the gas-phase ionization energy, to be calculated on a quantum-mechanical level. For electron or charge transfer states, analogous equations hold.

With a classical expansion of the molecular field and field response in terms of distributed multipoles (Sec. 3.2.1) and polarizabilities (Sec. 3.2.2) detailed above, $W_{s}$ follows from a variational principle, which replaces Poisson's equation $\nabla(\varepsilon \nabla \phi) \sim \rho$ in this microscopic, particle-based picture:

$$
\frac{\delta\left(W_{s}^{(1,2)}\right)}{\delta\left(\Delta Q_{l m}^{a(s)}\right)}=0 .
$$

Its self-consistent solution consists of the set of multipolar (here: dipolar) moments $\left\{\Delta Q_{l m}^{a(s)}\right\}$ induced at the (atomic) expansion sites $\{a\}$ with local polarizabilities $\left\{\alpha_{l m}^{a}\right\}$, in response to the permanent multipolar moments $\left\{Q_{l m}^{a(s)}\right\}$ that approximate the molecular, unperturbed charge densities. The set $\left\{\Delta Q_{l m}^{a(s)}\right\}$ constitutes the polarization state of the system, and together with $\left\{Q_{l m}^{a(s)}\right\}$ determines $W_{s}^{(1)}$ and $W_{s}^{(2)}$, and finally $\Delta_{s}^{(1,2)}$. We note that $W_{s}^{(2)}$, as opposed to $\Delta_{s}^{(2)}$, only takes negative values, as is characteristic of a second-order perturbative term. To illustrate how the $W_{s}$ 's are calculated, we decompose the self-interaction of a molecular system with a multipolar charge density $\mathcal{D}^{(s)}$ into an external and internal contribution, the 
latter corresponding to the (positive) polarization work:

$$
\begin{aligned}
& W_{\mathrm{ext}}^{(1,2)}\left[\mathcal{D}^{(s)} ; \mathcal{D}^{(s)}\right]=\frac{1}{2} \sum_{D^{(s)}} \sum_{D^{\prime(s)}}^{\prime}\left(Q_{t}^{d(s)}+\Delta Q_{t}^{d(s)}\right) T_{t u}^{d d^{\prime}}\left(Q_{u}^{d^{\prime}(s)}+\Delta Q_{u}^{d^{\prime}(s)}\right), \\
& W_{\mathrm{int}}^{(2)}\left[D^{(s)}\right]=\frac{1}{2} \sum_{D} \sum_{D^{\prime}} \delta_{D D^{\prime}} \Delta Q_{t}^{d(s)}\left(\alpha^{-1}\right)_{t t^{\prime}}^{d d^{\prime}(s)} \Delta Q_{t^{\prime}}^{d^{\prime}(s)} .
\end{aligned}
$$

Here, $\sum_{D}$ indicates a sum over molecules $D$ that participate in the density $\mathcal{D}$, with the prime over the sum indicating that terms with $D=D^{\prime}$ are excluded. Following Stone's notation [110], $Q_{t}$ and $Q_{u}$ are multipole moments in spherical-tensor representation, with angular and magnetic quantum numbers contracted into a single index. $T_{t u}^{d d^{\prime}}$ is the tensor that mediates the interaction between multipole moments $Q_{t}^{d}$ and $Q_{u}^{d^{\prime}}$ of $d \in D$ and $d^{\prime} \in D^{\prime}$, tabulated by Hattig et al. [118]. For both atomic indices and tensorial components, Einstein sum conventions are in place.

The classical expansion used above is of course not exhaustive. For instance, it neglects the intermolecular part of the nuclear polarization energy, which is, however, expected to be smaller than the intramolecular contribution of around $0.1 \mathrm{eV}$. Furthermore neglected are dispersive interactions: They are the primary contribution to the binding energy of organic solids, but have a negligible effect on site energies, since these are computed as the energy difference between an excited (charged) and ground (neutral) state. Finally, for computational efficiency, we truncate the expansion of the molecular charge density in the distributed multipole analysis at rank $l>2$, such that atomic quadrupoles are still accounted for. By using distributed polarizabilities in a local-dipole approximation, we disregard charge-flow effects [102]. In organic solids with their spatially rapidly fluctuating fields, charge flow is expected to play only a minor role; a local-dipole scheme is therefore sufficient to capture polarization effects in conjugated materials beyond the mean-field level.

We recall that - in order to avoid an unphysical overpolarization to which atomic-dipole schemes are susceptible - we have to damp induced-induced interactions at short separations. The interaction tensors $T_{t u}^{d d^{\prime}}$ in Eq. 3.30 are hence modified such that terms with a distance scaling of $R^{-\nu}$ are multiplied by a Thole damping function $\Lambda_{2 \nu+1}$ specified later in Sec. 3.4.3. Furthermore, due to the in comparison to biological molecules large polarizability of conjugated molecules, the set of Thole polarizabilities [115] has to be scaled (see Sec. 3.2.2) in order to match the volume of the polarizability ellipsoid $\left(\sim 1 / \Pi_{i=1}^{3} \tilde{\alpha}_{i}\right.$, where $\tilde{\alpha}_{i}$ is the $i$-th eigenvalue of the polarizability tensor) calculated via quantum-chemical methods.

\subsubsection{Aperiodic-Periodic Decomposition}

In the following, we detail how Eq. 3.29 is solved for localized, aperiodic excitations embedded in an otherwise periodic, neutral, polarizable environment, and how the associated perturbative contributions $\Delta_{s}^{(1)}$ and $\Delta_{s}^{(2)}$ are evaluated in a "long-range fashion" - i.e., with an infinite interaction range applied to all particles. Both tasks can be tackled with the same protocol 
in that they require the evaluation of fields $\varphi_{t}\left(\boldsymbol{r}_{p} ;\left[\mathcal{D}^{(s)}\right]\right)$ of order $t$ generated by an infinite, semiperiodic multipolar charge density $\mathcal{D}$.

First off, long-range interactions are of course not a recent topic in molecular simulations. A popular approach is based on the theory of Ewald [119], who partitioned the interaction potential onto two terms, one of which converges rapidly in real, the other in reciprocal space. This and derived techniques are commonly used in molecular dynamics simulations. To address the problem at hand, we readily extend the standard approach in three ways. First, we incorporate induction, which is not originally part of the formalism, but can be added [117]. Second, we address the long-range nature of the charge-quadrupole interaction. Third, we adapt the scope of the Ewald method to asymmetric, semiperiodic interaction densities. The molecular excitation itself, in particular, should not be periodically replicated in space. The resulting break in periodicity is due, first, to the uniqueness of the charged molecular cluster, second, to the induction response of the environment to the charged excitation. The accompanying polarization cloud is modelled by superimposing a non-periodic foreground onto a periodic, prepolarized, neutral background. The latter embodies the full solution of Eq. (3.29) for the neutral, ground-state system. The foreground not only incorporates the excitation, but also the entailed induction response. It can have non-zero charge depending on the nature of the excitation.

The polarization response to the excitation advises a decomposition of the periodic array of molecules $\mathcal{B}^{*}$ (see Fig. 3.4a), generated by replicas of the simulation box $\mathcal{B}=\mathcal{B}^{(n)}$, into a polarization cloud $\mathcal{P}^{(s)}$ and the background $\tilde{\mathcal{B}}^{*}=\mathcal{B}^{*} \backslash \mathcal{P}^{(n)}$. The configuration energy $W_{s}^{(i)}$ then reads

$$
W_{s}^{(i)}=W_{\mathrm{ext}}^{(i)}\left[\mathcal{P}^{(s)} ; \mathcal{P}^{(s)}\right]+W_{\mathrm{ext}}^{(i)}\left[\mathcal{P}^{(s)} ; \tilde{\mathcal{B}}^{*}\right]+W_{\mathrm{ext}}^{(i)}\left[\tilde{\mathcal{B}}^{*} ; \tilde{\mathcal{B}}^{*}\right]+W_{\mathrm{int}}^{(i)}\left[\mathcal{P}^{(s)}\right]+W_{\mathrm{int}}^{(i)}\left[\tilde{\mathcal{B}}^{*}\right]
$$

$W_{\text {ext }}$ and $W_{\text {int }}$ denote the intermolecular field interaction energy and intramolecular induction work (only defined for the second-order term), respectively, as defined in Eqs. 3.30 and 3.31. The polarization cloud (Fig. 3.4c) is chosen large enough to screen the excitation from $\tilde{\mathcal{B}}^{*}$, whose polarization state is thus assumed unaffected. Therefore no state index $s$ is used for the contributions $W_{\text {ext }}\left[\tilde{\mathcal{B}}^{*} ; \tilde{\mathcal{B}}^{*}\right]$ and $W_{\text {int }}\left[\tilde{\mathcal{B}}^{*}\right]$; they will cancel when taking the difference $\Delta_{s}^{(i)} \equiv W_{s}^{(i)}-W_{n}^{(i)}$ and hence need not be calculated. Based on Eqs. 3.30 and 3.31, the surviving terms are

$$
\begin{aligned}
& W_{\mathrm{ext}}^{(1,2)}\left[\mathcal{P}^{(s)} ; \mathcal{P}^{(s)}\right]=\frac{1}{2} \sum_{P} \sum_{P^{\prime}}\left(Q_{t}^{p(s)}+\Delta Q_{t}^{p(s)}\right) T_{t u}^{p p^{\prime}}\left(Q_{u}^{p^{\prime}}+\Delta Q_{u}^{p^{\prime}}\right), \\
& W_{\mathrm{ext}}^{(1,2)}\left[\mathcal{P}^{(s)} ; \tilde{\mathcal{B}}^{*}\right]=\sum_{P} \sum_{\tilde{B}^{*}}\left(Q_{t}^{p(s)}+\Delta Q_{t}^{p(s)}\right) T_{t u}^{p b}\left(Q_{u}^{b}+\Delta Q_{u}^{b}\right), \\
& W_{\mathrm{int}}^{(2)}\left[\mathcal{P}^{(s)}\right]=\frac{1}{2} \sum_{P} \Delta Q_{t}^{p(s)}\left(\alpha^{-1}\right)_{t}^{a(s)} \Delta Q_{t}^{p(s)} .
\end{aligned}
$$

In these expressions, induced moments have to be calculated self-consistently on the basis of 
Eq. 3.29. The resulting linear system of equations for $\Delta Q_{t}^{p(s)}$ reads

$$
\Delta Q_{t}^{p(s)}=-\sum_{\tilde{B}^{*}} \alpha_{t}^{p(s)} T_{t u}^{p b}\left(Q_{u}^{b}+\Delta Q_{u}^{b}\right)-\sum_{P} \alpha_{t}^{p(s)} T_{t t^{\prime}}^{p p^{\prime}} \Delta Q_{t^{\prime}}^{p^{\prime}(s)}
$$

and is best solved iteratively via successive over-relaxation $[110,117]$. Note that in Eqs. 3.33 and 3.35, $W_{\text {ext }}^{(i)}\left[\mathcal{P}^{(s)} ; \mathcal{P}^{(s)}\right]$ and $W_{\text {int }}^{(i)}\left[\mathcal{P}^{(s)}\right]$ only count interactions within $\mathcal{P}^{(s)}$, such that direct evaluation is possible at reasonable computational expense. $W_{\text {ext }}^{(i)}\left[\mathcal{P}^{(s)} ; \tilde{\mathcal{B}}^{*}\right]$, however, comprises the interaction of $\mathcal{P}^{(s)}$ with the infinite, foreground-subtracted (hence semiperiodic) set $\tilde{\mathcal{B}}^{*}$; this renders it intractable by direct evaluation of the interaction sum.

A transformation of $W_{\text {ext }}^{(i)}\left[\mathcal{P}^{(s)} ; \tilde{\mathcal{B}}^{*}\right]$, based on an aperiodic-periodic-type decomposition, is therefore needed: To this end, the foreground density in its neutral charge and polarization state $\mathcal{P}^{(n)}$ is again added to $\tilde{\mathcal{B}}^{*}$; the potentials generated by the resulting periodic charge density $\mathcal{B}^{*}$ can be computed efficiently after an Ewald transformation, which starts from the decomposition of the $1 / r$ charge-charge interaction into $1 / r=\operatorname{erf}(\beta r) / r+\operatorname{erfc}(\beta r) / r$. Here, the first term absorbs the long-range contribution to be evaluated in reciprocal space, following a Fourier transformation of the sum over periodic images. The second term absorbs the shortrange part of the Coulomb potential, and can be treated in real space. The parameter $\beta$ can be tuned to achieve fast convergence of both reciprocal-space and real-space interaction sums. Higher-order fields and interaction tensors follow from taking spatial derivatives with regard to the coordinates of the source and target atomic positions. $W_{\text {ext }}^{(i)}\left[\mathcal{P}^{(s)} ; \tilde{\mathcal{B}}^{*}\right]$ is then obtained as the sum over five contributions (the order superscript $(i)$ will from here on be omitted),

$$
\begin{gathered}
W_{\text {ext }}\left[\mathcal{P}^{(s)} ; \tilde{\mathcal{B}}^{*}\right]=W_{r}\left[\mathcal{P}^{(s)} ; \tilde{\mathcal{B}}^{*}\right]+W_{k}\left[\mathcal{P}^{(s)} ; \mathcal{B}\right]-W_{c}^{\prime}\left[\mathcal{P}^{(s)} ; \mathcal{P}^{(n)}\right]- \\
-W_{c}\left[\mathcal{P}^{(s)} ; \mathcal{P}^{(n)}\right]+W_{*}\left[\mathcal{P}^{(s)} ; \mathcal{B}\right]
\end{gathered}
$$

We will now discuss this decomposition in greater detail, as it is a key equation within the scheme, in addition to an analogous expression that holds for the computation of molecular fields (see Sec. 3.4.4).

The first three contributions in Eq. 3.37 are the standard Ewald terms for the real$\left(W_{r}\right)$ and reciprocal-space $\left(W_{k}\right)$ interaction, and self-interaction correction $\left(W_{c}^{\prime}\right)$, respectively. Their interaction kernels take into account higher-order permanent and induced moments $[120,121]$ and, in the case of $W_{r}\left[\mathcal{P}^{(s)} ; \tilde{\mathcal{B}}^{*}\right]$, incorporate short-range damping functions $\Lambda_{2 \nu+1}$ as discussed above. These first three terms read

$$
\begin{aligned}
W_{r}\left[\mathcal{P}^{(s)} ; \tilde{\mathcal{B}}^{*}\right] & =\frac{1}{4 \pi \varepsilon_{0}} \sum_{\tilde{B}^{*}} \sum_{\mathcal{P}^{(s)}} \sum_{\nu=0}^{4} \mathcal{T}_{\nu}^{p b} \Lambda_{2 \nu+1}^{p b} \mathcal{B}_{\nu}\left(R_{L}^{p b}\right) \\
W_{k}\left[\mathcal{P}^{(s)} ; \mathcal{B}\right] & =\frac{1}{4 \pi \varepsilon_{0}} \frac{4 \pi}{V} \sum_{\boldsymbol{k} \neq 0}^{\infty} \mathcal{S}\left(\boldsymbol{k} ;\left[\mathcal{P}^{(s)}\right]\right) \mathcal{S}^{*}\left(\boldsymbol{k} ;\left[\mathcal{B}^{*}\right]\right) \mathcal{A}(k) \\
W_{c}^{\prime}\left[\mathcal{P}^{(s)} ; \mathcal{P}^{(n)}\right] & =\frac{1}{4 \pi \varepsilon_{0}} \sum_{\mathcal{P}}\left(\frac{2 \beta}{\sqrt{\pi}} q^{p(s)} q^{p(n)}+\frac{4 \beta^{3}}{3 \sqrt{\pi}} \boldsymbol{\mu}^{p(s)} \cdot \boldsymbol{\mu}^{p(n)}+\frac{16 \beta^{5}}{5 \sqrt{\pi}} \tilde{\theta}^{p(s)}: \tilde{\theta}^{p(n)}\right) .
\end{aligned}
$$


For the real- and reciprocal-space terms, the anisotropic interaction kernels $\mathcal{T}_{\nu}^{p b}$ and $\mathcal{S}$ (structure factor), listed in Sec. 3.4.1, describe the orientation dependence of the interaction between two sets of atomic multipoles in the respective space. The associated isotropic interaction kernels $A(k)$ and $B_{\nu}(R)$ (see Sec. 3.4.2) yield the distance dependence. Finally, the realspace interaction is damped by the damping function $\Lambda_{2 \nu+1}$ prescribed by $B_{\nu}(R)$, which scales as $R^{-(2 \nu+1)}$ for sufficiently small distances (see Sec. 3.4.3 for details). In the above equations, the set of atom-centred multipoles incorporates the atomic charge $q$, dipole $\boldsymbol{\mu}$ and quadrupole $\tilde{\theta}$, now in their Cartesian representation. Note that the quadrupole is defined as $\theta_{\alpha \beta}=\sum\left(\frac{1}{2} r_{\alpha} r_{\beta}-\frac{1}{6} \delta_{\alpha \beta} r^{2}\right)$, which differs from the conventional definition by a factor $\frac{1}{3}$. Also note that the sum $\sum_{\tilde{B}^{*}}$ is implemented as a double sum over image-box vectors $\boldsymbol{L}$ and the periodic density $\mathcal{B}^{*}$, with molecules participating in the polarization cloud $\mathcal{P}^{(s)}$ and $\mathcal{P}^{(n)}$ excluded.

The fourth term in Eq. 3.37, $W_{c}\left[\mathcal{P}^{(s)} ; \mathcal{P}^{(n)}\right]$, denotes an aperiodic subtraction which corrects for the overlap between $\mathcal{P}^{(s)}$ and $\mathcal{B}^{*} \supset \mathcal{P}^{(n)}$,

$$
W_{c}\left[\mathcal{P}^{(s)} ; \mathcal{P}^{(n)}\right]=\frac{1}{4 \pi \varepsilon_{0}} \sum_{P^{(s)}} \sum_{P^{(n)}}^{\prime} \sum_{\nu=0}^{4} \mathcal{T}_{\nu}^{p p^{\prime}} \mathcal{C}_{\nu}\left(R_{0}^{p p^{\prime}}\right)
$$

Here, the anisotropic kernel is identical to $\mathcal{T}$ from Eq. 3.38. The isotropic kernel $\mathcal{C}_{\nu}$, however, differs from $\mathcal{B}_{\nu}$ (see Sec. 3.4.2) in that it involves derivatives of the long-ranged $\operatorname{erf}(\beta r) / r$ rather than the short-ranged $\operatorname{erfc}(\beta r) / r$ part of the interaction.

\subsubsection{Shape Terms}

The fifth term in Eq. 3.37, $W_{s}^{*}\left[\mathcal{P}^{(s)} ; \mathcal{B}^{*}\right]$, is a shape $(k=0)$ contribution that tackles the conditionality of the interaction sum. An analogous conditionality arises in overall neutral systems with a net dipole moment of the periodically repeated charge density $\mathcal{B}$ [122], as is often encountered in molecular systems. As a result, the convergence of the interaction sum depends on the (macroscopic) summation shape. Here, we address molecular solids, where the conditionality arises from the net charge that resides in $\mathcal{P}^{(s)}$ and interacts with a net-quadrupolar background. To take this into account, shape terms for summation of the simulation cell into a (macroscopic) slab or cube structure are derived as

$$
\begin{aligned}
& W_{*}\left[\mathcal{P}^{(s)} ; \mathcal{B}\right]=-\frac{1}{4 \pi \varepsilon_{0}} \frac{4 \pi}{3 V}\left(Q_{0}^{\mathcal{P}(s)} \operatorname{Tr}\left[\tilde{\Theta}^{\mathcal{B}(n)}\right]+Q_{0}^{\mathcal{B}(n)} \operatorname{Tr}\left[\tilde{\Theta}^{\mathcal{P}(s)}\right]-M^{\mathcal{P}(s)} \cdot M^{\mathcal{B}(n)}\right), \\
& W_{*}\left[\mathcal{P}^{(s)} ; \mathcal{B}\right]=-\frac{1}{4 \pi \varepsilon_{0}} \frac{4 \pi}{V}\left(Q_{0}^{\mathcal{P}(s)} \Theta_{z z}^{\mathcal{B}(n)}+Q_{0}^{\mathcal{B}(n)} \Theta_{z z}^{\mathcal{P}(s)}-M_{z}^{\mathcal{P}(s)} M_{z}^{\mathcal{B}(n)}\right)
\end{aligned}
$$

where Eq. 3.41 implies summation over cubic, and Eq. 3.42 over (infinitely thin) slab-shaped shells. The net multipolar moments of the densities $\mathcal{P}^{(s)}$ and $\mathcal{B}$ that feature in these expres- 


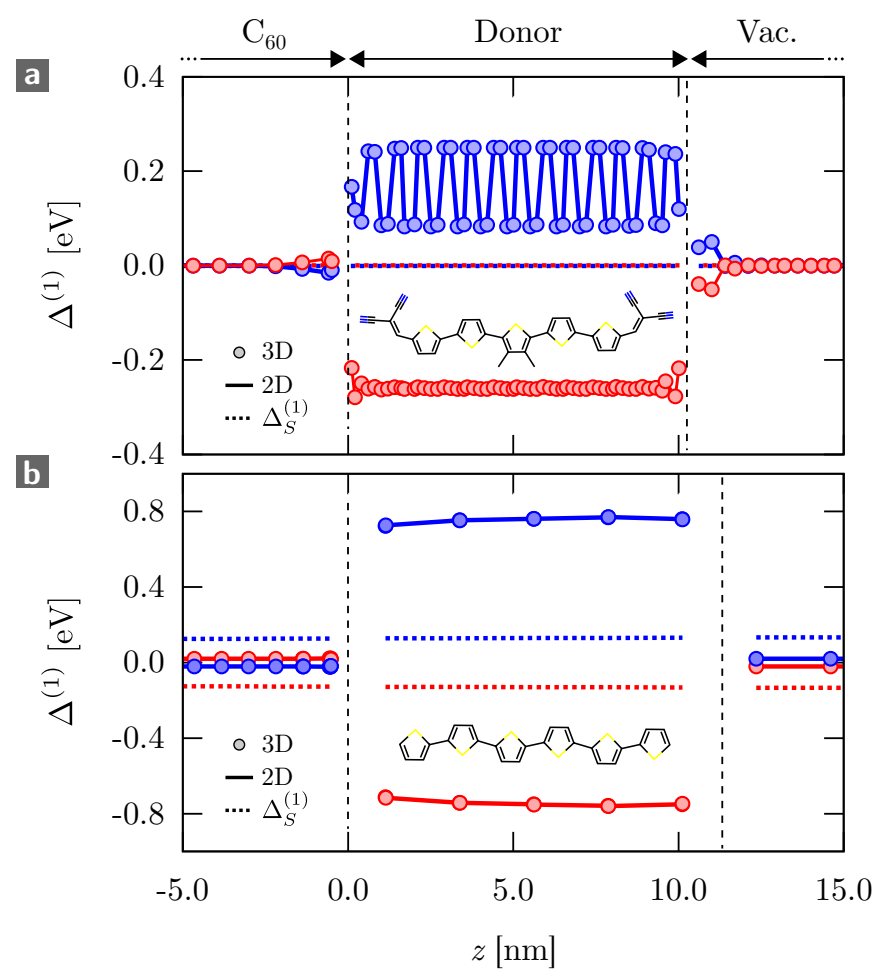

Figure 3.3. 2D- versus 3D-periodic description of thin films. Electrostatic energy level profile $\Delta^{(1)}(z)$ across a D5M (a) and $6 \mathrm{~T}$ (b) $10 \mathrm{~nm}$ thin film for holes (red) and electrons (blue), once computed in a 2D-periodic (lines), and once in a 3D-periodic framework under application of the appropriate thin-film shape term from Eq. 3.42 (symbols). Dashed blue and red lines mark the contribution from this shape term, addition of which leads to perfect agreement between the 2D- and 3D-periodic description.

sions are defined as

$$
\begin{aligned}
Q_{0}^{\mathcal{P}(s)} & =\sum_{P} q^{p(s)}, \\
\boldsymbol{M}^{\mathcal{P}(s)} & =\sum_{P}\left(q^{p(s)} \boldsymbol{r}_{p}+\boldsymbol{\mu}^{p(s)}\right) \\
\tilde{\Theta}^{\mathcal{P}(s)} & =\sum_{P}\left(\frac{1}{2} q^{p(s)} \boldsymbol{r}_{p} \otimes \boldsymbol{r}_{p}+\boldsymbol{\mu}^{p(s)} \otimes \boldsymbol{r}_{p}+\tilde{\theta}^{p(s)}\right) .
\end{aligned}
$$

Analogous expressions are used for $Q_{0}^{\mathcal{B}}, \boldsymbol{M}^{\mathcal{B}}$ and $\Theta^{\mathcal{B}}$.

The conditionality that arises from the charge-quadrupole interaction in the case of 3Dinfinite systems is lifted for lower dimensions. The scheme detailed above is, however, built on a 3D-periodic description, and hence relies on suitably chosen shape corrections. This 3Dperiodic description is still preferred, first, for efficiency (the reciprocal-space sum conveniently factorizes with respect to the two multipolar densities $\mathcal{P}^{(s)}$ and $\mathcal{B}$ ), second, for flexibility (it 
enables the simulation of both thin-film and bulk conditions). For organic electronic devices, which are typically structured into functional layers, a thin-film description is advised. We will therefore show that the slab-like limit from Eq. 3.42 is indeed suited to mimic a 2Dperiodic setup, with periodicity along the thin-film normal (aligned with the $z$-axis) effectively removed. To this end, we compare electrostatic level profiles $\Delta^{(1)}(z)$ across a $\mathrm{C}_{60} / \mathrm{D} 5 \mathrm{M}$ and $\mathrm{C}_{60} / 6 \mathrm{~T}$ interface, obtained once with the 3D-periodic framework detailed above and once with a 2D-periodic framework [123, 124].

In the 2D-periodic description, the potential is calculated according to the standard Ewaldtype decomposition $[123,124]$

$$
\phi\left(\boldsymbol{r}_{p},\left[\tilde{\mathcal{B}}^{*}\right]\right)=\phi_{r}\left(\boldsymbol{r}_{p},\left[\tilde{\mathcal{B}}^{*}\right]\right)+\phi_{k}\left(\boldsymbol{r}_{p},\left[\mathcal{B}^{*}\right]\right)-\phi_{c}\left(\boldsymbol{r}_{p},\left[\mathcal{P}^{(n)}\right]\right) .
$$

Shape contributions are explicitly included in the $(k=0)$-term of $\phi_{k}\left(\boldsymbol{r}_{p},\left[\mathcal{B}^{*}\right]\right)$, where chargequadrupole contributions are exempt, as these prove absolutely convergent in 2D-periodic systems. The individual contributions to Eq. 3.46 hence read

$$
\begin{aligned}
\phi_{r}\left(\boldsymbol{r}_{p},\left[\tilde{\mathcal{B}}^{*}\right]\right) & =\frac{1}{4 \pi \varepsilon_{0}} \sum_{\tilde{B}^{*}} \frac{q^{b(n)} \operatorname{erfc}\left(\beta R_{L}^{p b}\right)}{R_{L}^{p b}}, \\
\phi_{k}\left(\boldsymbol{r}_{p},\left[\mathcal{B}^{*}\right]\right) & =\frac{1}{4 \pi \varepsilon_{0}} \sum_{\boldsymbol{k} \neq \mathbf{0}}^{\prime} \sum_{B^{*}} \frac{2 \pi q^{b(n)}}{\left|\boldsymbol{L}_{a} \times \boldsymbol{L}_{b}\right|} \cos \left(\boldsymbol{k} \cdot \boldsymbol{R}^{p b}\right)\left[e^{-k\left(z_{p}-z_{b}\right)} \operatorname{erfc}\left(\frac{k}{2 \beta}-\beta\left(z_{p}-z_{b}\right)\right)+\right. \\
& \left.+e^{+k\left(z_{p}-z_{b}\right)} \operatorname{erfc}\left(\frac{k}{2 \beta}+\beta\left(z_{p}-z_{b}\right)\right)\right]-\frac{1}{4 \pi \varepsilon_{0}} \sum_{B^{*}} \frac{2 \pi q^{b(n)}}{\left|\boldsymbol{L}_{a} \times \boldsymbol{L}_{b}\right|}\left[\frac{e^{-\beta^{2}\left(z_{a}-z_{b}\right)^{2}}}{\sqrt{\pi} \beta}+\right. \\
& \left.+\left(z_{a}-z_{b}\right) \operatorname{erf}\left(\beta\left(z_{a}-z_{b}\right)\right)\right], \\
\phi_{c}\left(\boldsymbol{r}_{p},\left[\mathcal{P}^{(n)}\right]\right) & =\frac{1}{4 \pi \varepsilon_{0}} \sum_{P} \frac{q^{p(n)} \operatorname{erf}\left(\beta R_{L}^{p b}\right)}{R_{l}^{p b}} .
\end{aligned}
$$

$\boldsymbol{L}_{a}, \boldsymbol{L}_{b}$ are the simulation-cell vectors in the periodic plane. Note that self-interaction terms with $R_{L}^{p b}=0$ in $\phi_{c}\left(\boldsymbol{r}_{p},\left[\mathcal{P}^{(n)}\right]\right)$ are calculated using the limit $\operatorname{erf}\left(\beta R_{L}^{p b}\right) / R_{L}^{p b} \rightarrow 2 \beta / \sqrt{\pi}$ as $R_{L}^{p b} \rightarrow 0$.

As can be seen in Fig. 3.3, the results for both approaches (2D- and 3D-periodic, indicated via dots and lines, respectively) coincide perfectly, if the shape contribution (which is nonzero for the case of $6 \mathrm{~T}$, see the dotted lines) is taken into account. The shape term from Eq. 3.42 should hence by no means be neglected in simulations that target $2 \mathrm{D}$ properties in meso-ordered systems. 


\subsection{Interaction Kernels}

To specify the particle-particle interaction kernels referenced in Sec. 3.3.2 above, we use the following definitions: $\boldsymbol{R}_{L}^{a b}=\boldsymbol{r}_{a}-\boldsymbol{r}_{b}+\boldsymbol{L}$ denotes the image-box-vector $(\boldsymbol{L})$ corrected particle-particle interaction vector. The outer products of real- and reciprocal-space vectors are abbreviated via $\mathbb{R}=\boldsymbol{R} \otimes \boldsymbol{R}$ and $\mathbb{K}=\boldsymbol{k} \otimes \boldsymbol{k}$, respectively. The dyadic product between matrices $M$ and $N$ is written as $M: N$. Finally, the atomic quadrupole is defined as $\tilde{\theta}_{\alpha \beta}=$ $\sum\left(\frac{1}{2} r_{\alpha} r_{\beta}-\frac{1}{6} \delta_{\alpha \beta} r^{2}\right)$, which avoids prefactors in the interaction kernels.

\subsubsection{Anisotropic Kernels}

In real space, the $\mathcal{T}_{\nu}^{p b}$ symbols from Eq. 3.38 describe the orientation dependence of the interaction between polar sites $p$ and $b$ with charge $q$, dipole $\boldsymbol{\mu}$ and quadrupole $\tilde{\theta}$ (in Cartesian representation, see the definition above). They read

$$
\begin{aligned}
\mathcal{T}_{0}^{p b} & =q^{p} q^{b}, \\
\mathcal{T}_{1}^{p b} & =\boldsymbol{\mu}^{p} \cdot \boldsymbol{\mu}^{b}+\left(q^{p} \boldsymbol{\mu}^{b}-q^{b} \boldsymbol{\mu}^{p}\right) \cdot \boldsymbol{R}^{p b}, \\
\mathcal{T}_{2}^{p b} & =2 \tilde{\Theta}^{p}: \tilde{\Theta}^{b}+\left(q^{p} \tilde{\Theta}^{b}+q^{b} \tilde{\Theta}^{p}\right): \mathbb{R}^{p b}-\left(\boldsymbol{\mu}^{p} \cdot \boldsymbol{R}^{p b}\right)\left(\boldsymbol{\mu}^{b} \cdot \boldsymbol{R}^{p b}\right)+ \\
& -2\left(\boldsymbol{\mu}^{b} \otimes \boldsymbol{R}^{p b}\right): \tilde{\Theta}^{p}+2\left(\boldsymbol{\mu}^{p} \otimes \boldsymbol{R}^{p b}\right): \tilde{\Theta}^{b}, \\
\mathcal{T}_{3}^{p b} & =-4\left(\tilde{\Theta}^{p} \cdot \boldsymbol{R}^{p b}\right)\left(\tilde{\Theta}^{b} \cdot \boldsymbol{R}^{p b}\right)-\left(\boldsymbol{\mu}^{p} \cdot \boldsymbol{R}^{p b}\right)\left(\tilde{Q}^{b}: \mathbb{R}^{p b}\right)+ \\
& +\left(\boldsymbol{\mu}^{b} \cdot \boldsymbol{R}^{p b}\right)\left(\tilde{Q}^{p}: \mathbb{R}^{p b}\right), \\
\mathcal{T}_{4}^{p b} & =\left(\tilde{Q}^{p}: \mathbb{R}^{p b}\right)\left(\tilde{Q}^{b}: \mathbb{R}^{p b}\right) .
\end{aligned}
$$

The $k$-space anisotropy is absorbed in the structure factors $\mathcal{S}$ of the multipolar charge density, featuring in Eq. 3.39:

$$
\mathcal{S}\left(\boldsymbol{k} ;\left[\mathcal{P}^{(s)}\right]\right)=\sum_{P}\left(q^{p}+i \boldsymbol{\mu}^{p} \cdot \boldsymbol{k}-\tilde{\Theta}^{p}: \mathbb{K}\right) \exp \left(i \boldsymbol{k} \cdot \boldsymbol{r}_{p}\right)
$$

Note that the dipole moment $\boldsymbol{\mu}$ in the above expressions for $\mathcal{T}_{\nu}^{p b}$ and $\mathcal{S}$ also incorporates the induced moment $\Delta \boldsymbol{\mu}$. If an energy splitting in terms of electrostatic and polarization contributions is desired, the $\mathcal{T}_{\nu}^{p b}$ symbols, as well as $\mathcal{S}$ have be decomposed accordingly.

\subsubsection{Isotropic Kernels}

The distance dependence of the interaction in Eqs. 3.39, 3.38, 3.40 is described by the isotropic kernels $\mathcal{A}(k), \mathcal{B}_{\nu}(R)$ and $\mathcal{C}_{\nu}(R)$, respectively, next to powers of $k$ and $R$ that already appear in the scalar contractions of $\mathcal{S}$ and $\mathcal{T}_{\nu}^{p b}$ above:

$$
\begin{aligned}
\mathcal{A}(k) & =k^{-2} \exp \left(-\frac{k^{2}}{4 \beta^{2}}\right), \\
\mathcal{B}_{0}(R) & =R^{-1} \operatorname{erfc}(\beta R),
\end{aligned}
$$




$$
\begin{aligned}
& \mathcal{B}_{\nu}(R)=R^{-2}\left[(2 \nu-1) B_{\nu-1}(R)+\frac{2^{\nu} \beta^{2 \nu-1}}{\sqrt{\pi}} \exp \left(-\beta^{2} R^{2}\right)\right], \\
& \mathcal{C}_{0}(R)=R^{-1} \operatorname{erf}(\beta R), \\
& \mathcal{C}_{\nu}(R)=R^{-2}\left[(2 \nu-1) C_{\nu-1}(R)-\frac{2^{\nu} \beta^{2 \nu-1}}{\sqrt{\pi}} \exp \left(-\beta^{2} R^{2}\right)\right] .
\end{aligned}
$$

\subsubsection{Thole Damping Kernels}

The Thole model damps induced-induced interactions at short distances. For faster convergence, damping of induced-permanent interactions - though not mandatory - may also be advised. With the polarizability $\tilde{\alpha}$ (here written more generally as a tensor quantity), an effective interaction distance is defined as

$$
u^{p b}(R)=\left(\frac{\tilde{\alpha}^{p}: \tilde{\alpha}^{b}}{3}\right)^{-1 / 6} R .
$$

With an exponential smearing function $\sim \exp \left(-\gamma u^{3}\right)$ as used in the AMOEBA force field [117], where $\gamma=0.39$, the damping functions $\Lambda_{2 \nu+1}$ are

$$
\begin{aligned}
& \Lambda_{3}(R)=1-\exp \left[-\gamma u^{p b}(R)^{3}\right], \\
& \Lambda_{5}(R)=1-\left[1+\gamma u^{p b}(R)^{3}\right] \exp \left[-\gamma u^{p b}(R)^{3}\right], \\
& \Lambda_{7}(R)=1-\left[1+\gamma u^{p b}(R)^{3}+\frac{3}{5} \gamma^{2} u^{p b}(R)^{6}\right] \exp \left[-\gamma u^{p b}(R)^{3}\right], \\
& \Lambda_{9}(R)=1-\left[1+\gamma u^{p b}(R)^{3}+\frac{18}{35} \gamma^{2} u^{p b}(R)^{6}+\frac{9}{35} \gamma^{3} u^{p b}(R)^{9}\right] \exp \left[-\gamma u^{p b}(R)^{3}\right] .
\end{aligned}
$$

As higher orders of the damped interaction tensors are obtained from derivatives of tensors of lower order, the damping functions can be applied such that interaction terms that scale as $\frac{R_{\alpha} \cdots R_{\beta}}{R^{n}}$ (with $\alpha, \beta \in\{x, y, z\}$ ) are damped by $\Lambda_{n}$; or, put simpler, terms of power $-n$ in the isotropic distance $R$ are damped by $\Lambda_{n}$. For the real-space interaction from Eq. 3.38, the damping is hence prescribed by the isotropic kernel $B_{\nu}$, which scales as $R^{-(2 \nu+1)}$ for $R \ll 1 / \beta$. For the $C_{\nu}$, no damping is required as long as the convergence parameter $\beta$ is sufficiently small $(1 / \beta \gtrsim \mathrm{nm})$, since we are then dealing with a purely long-ranged compensation term.

Special care has to be taken for the spherical interaction tensors $T_{t u}^{p p^{\prime}}$ from Eq. 3.33, where different summands appearing in $T$ have to be multiplied by a different $\Lambda_{2 \nu+1}$ according to the rule above.

\subsubsection{Field Calculation}

For the evaluation of electric fields required in the polarization cycle (Eq. 3.36), a separate set of interaction kernels applies. We again start from a decomposition of the external fields 
analogous to Eq. 3.37:

$$
\begin{aligned}
\boldsymbol{E}\left(\boldsymbol{r}_{p},\left[\tilde{\mathcal{B}}^{*}\right]\right) & =\boldsymbol{E}_{r}\left(\boldsymbol{r}_{p},\left[\tilde{\mathcal{B}}^{*}\right]\right)+\boldsymbol{E}_{k}\left(\boldsymbol{r}_{p},\left[\mathcal{B}^{*}\right]\right)-\boldsymbol{E}_{c}^{\prime}\left(\boldsymbol{r}_{p},\left[\mathcal{P}^{(n)}\right]\right)- \\
& -\boldsymbol{E}_{c}\left(\boldsymbol{r}_{p},\left[\mathcal{P}^{(n)}\right]\right)+\boldsymbol{E}_{s}^{*}\left(\boldsymbol{r}_{p},\left[\mathcal{B}^{*}\right]\right) .
\end{aligned}
$$

The individual terms correspond to the real-space $\left(\boldsymbol{E}_{r}\right)$ and reciprocal-space contribution $\left(\boldsymbol{E}_{k}\right)$, self-interaction correction $\left(\boldsymbol{E}_{c}^{\prime}\right)$, aperiodic subtraction $\left(\boldsymbol{E}_{c}\right)$ and shape contribution $\left(\boldsymbol{E}_{s}\right)$, respectively:

$$
\begin{aligned}
\boldsymbol{E}_{r}\left(\boldsymbol{r}_{p},\left[\tilde{\mathcal{B}}^{*}\right]\right) & =-\frac{1}{4 \pi \varepsilon_{0}} \sum_{\tilde{B}^{*}} \sum_{\nu=1}^{3} \mathcal{T}_{\nu}^{p b} \Lambda_{2 \nu+1}^{p b} \mathcal{B}_{\nu}\left(R_{L}^{p b}\right) \\
\boldsymbol{E}_{k}\left(\boldsymbol{r}_{p},\left[\mathcal{B}^{*}\right]\right) & =-\frac{1}{4 \pi \varepsilon_{0}} \frac{4 \pi}{V} \sum_{\boldsymbol{k} \neq 0}^{\infty} i \boldsymbol{k} \exp \left(i \boldsymbol{k} \cdot \boldsymbol{r}_{p}\right) A(k) \mathcal{S}^{*}\left(\boldsymbol{k} ;\left[\mathcal{B}^{*}\right]\right), \\
\boldsymbol{E}_{c}\left(\boldsymbol{r}_{p},\left[\mathcal{P}^{(n)}\right]\right) & =-\frac{1}{4 \pi \varepsilon_{0}} \sum_{P^{(n)}}^{\prime} \sum_{\nu=1}^{3} \mathcal{T}_{\nu}^{p b} \Lambda_{2 \nu+1}^{p b} \mathcal{B}_{\nu}\left(R_{L}^{p b}\right) \\
\boldsymbol{E}_{c}^{\prime}\left(\boldsymbol{r}_{p},\left[\mathcal{P}^{(n)}\right]\right) & =-\frac{1}{4 \pi \varepsilon_{0}} \frac{4 \alpha^{3}}{3 \sqrt{\pi}} \boldsymbol{\mu}^{p}
\end{aligned}
$$

The vector symbols $\mathcal{T}$ absorb the orientation dependence of the interaction,

$$
\begin{aligned}
& \mathcal{T}_{1}^{p b}=-q_{b} \boldsymbol{R}^{p b}+\boldsymbol{\mu}^{b}, \\
& \mathcal{T}_{2}^{p b}=2 \tilde{\Theta}^{b} \cdot \boldsymbol{R}^{p b}-\left(\boldsymbol{\mu}^{b} \cdot \boldsymbol{R}^{p b}\right) \boldsymbol{R}^{p b}, \\
& \mathcal{T}_{3}^{p b}=-\left(\tilde{\Theta}^{b}: \mathbb{R}\right) \boldsymbol{R}^{p b} .
\end{aligned}
$$

The conditionality in the field calculation is removed through application of the appropriate shape term,

$$
\begin{aligned}
& \boldsymbol{E}_{s}\left(\boldsymbol{r}_{p},\left[\mathcal{B}^{*}\right]\right)=-\frac{1}{4 \pi \varepsilon_{0}} \frac{4 \pi}{3 V} \boldsymbol{M}^{\mathcal{B}(n)}, \\
& \boldsymbol{E}_{s}\left(\boldsymbol{r}_{p},\left[\mathcal{B}^{*}\right]\right)=-\frac{1}{4 \pi \varepsilon_{0}} \frac{4 \pi}{V} M_{z}^{\mathcal{B}(n)} \hat{e}_{z},
\end{aligned}
$$

where the former expression corresponds to a cubic limit, the latter to a thin-film limit with vanishing thickness.

\subsection{Computational Procedure for Site-Energy Calculations}

Combining our results for energy, field and polarization calculations, Fig. 3.4 summarises the four-step procedure to solve Eq. 3.29 and compute $W_{s}^{(1,2)}$ for a molecular system in a state s. First (Fig. 3.4a), the polarization state of the ground-state system is computed according to Eq. 3.36 above and Eq. 3.66. In the case of site-energy calculations, this prepolarization 


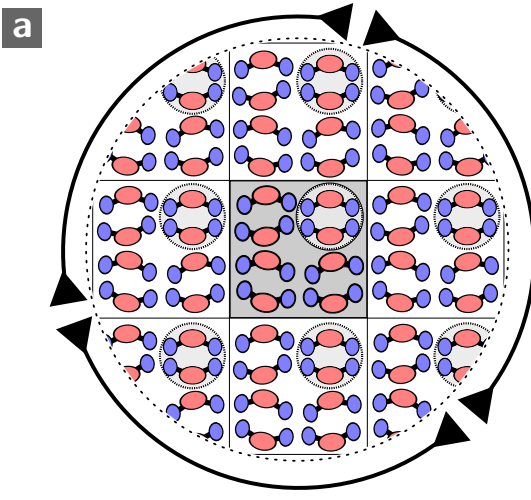

background polarization

C

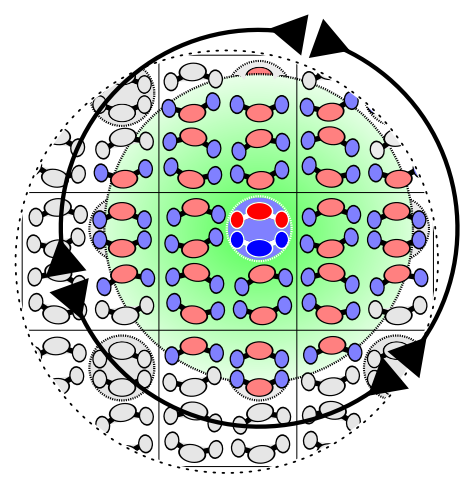

polarization cloud b

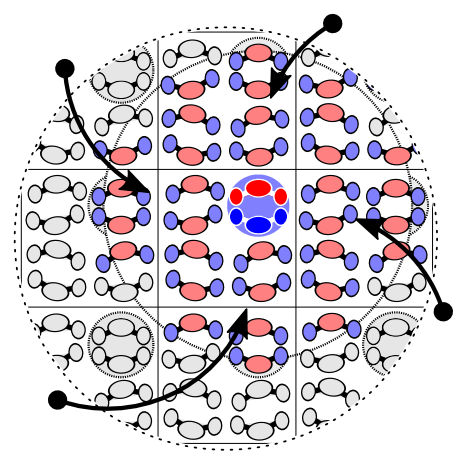

foreground fields

d

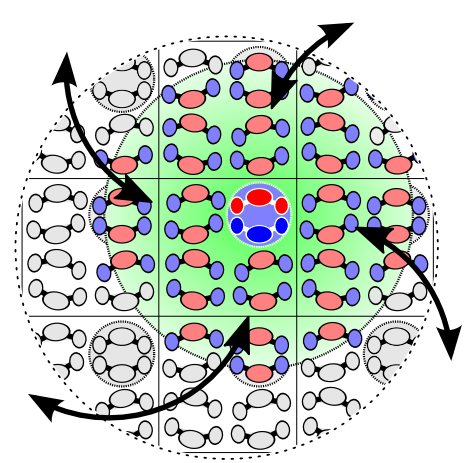

background interaction

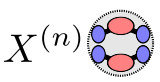

$X^{(s)}$

$P^{(s)}$

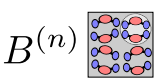

Figure 3.4. Four-step embedding workflow. (a) Step 1: Computation of the polarization state of the periodic ground-state system. The simulation box $B^{(n)}$, coloured in grey, incorporates the molecular cluster under study, with a ground-state multipolar charge density $X^{(n)}$. (b) Step 2: Excitation of the molecular cluster and calculation of background fields acting on the polarization cloud (foreground) $P^{(s)}$ centred around the excited cluster $X^{(s)}$. (c) Step 3: Selfconsistent polarization within $P^{(s)}$ and evaluation of the self-energy of $P^{(s)}$. (d) Step 4: Evaluation of the interaction energy between the foreground $P^{(s)}$ and the polarized background $\tilde{\mathcal{B}}^{*}$. 
only needs to be carried out once for each coordinate configuration. Second (Fig. 3.4b), fields generated by the semiperiodic background across the polarization cloud are calculated, taking into account both permanent and induced moments. Third (Fig. 3.4c), the polarization cloud is polarized self-consistently. Fourth (Fig. 3.4d), the interaction-energy between the semiperiodic background and the polarization cloud, as well as within the polarization cloud, are evaluated using Eqs. 3.33, 3.35 and 3.37.

The computational cost of the above procedure is mostly due to the self-consistent polarization of the polarization cloud (step 3). The cost of this step scales as $r_{p c}^{6}$ with the radius $r_{p c}$ of the cloud. Typically, $r_{p c}=4 \mathrm{~nm}$ proves to be sufficient to converge level profiles, neglecting, however, the residual dielectric response of the material beyond $r_{p c}$. The latter can nevertheless be recovered in an $a d-h o c$ fashion [125]: To this end, we consider the polarization energy of a charge localized in a non-polarizable spherical cavity with radius $r_{p c}$, embedded in a film of dielectric constant $\varepsilon_{1}$ in the half-space $z>0$, in the vicinity of an interface with a second dielectric layer of dielectric constant $\varepsilon_{2}$ in $z<0$ (a situation frequently encountered in organic electronic devices):

$$
\Delta_{s}^{(\varepsilon)}\left(z>r_{p c}\right)=-\frac{1}{8 \pi \varepsilon_{0}} \frac{q^{2}}{r_{p c}} \frac{\varepsilon_{1}-1}{\varepsilon_{1}}\left[1+\frac{r_{p c}}{2 z} \frac{\varepsilon_{2}-\varepsilon_{1}}{\left(\varepsilon_{1}-1\right)\left(\varepsilon_{1}+\varepsilon_{2}\right)}\right] .
$$

This expression holds for excitations with a net-charge $q$ as leading moment. Higher order moments, as they would apply to net-neutral excitations, in particular charge transfer states with an in-built dipole, could be readily treated on the same grounds [125]. In practice, the contribution from higher-order moments is, however, negligible - different from charge carriers, where, as a numerical example, $\Delta^{(\varepsilon)}$ evaluates to $-0.11 \mathrm{eV}$ for a hole or electron embedded in an environment of $\varepsilon_{1}=\varepsilon_{2}=3$ and $r_{p c}=4 \mathrm{~nm}$. Eq. 3.76 also captures the contribution to level bending that results from a difference in dielectric constant on two sides of an interface (second term in brackets). For organic semiconductors, with dielectric constants contained in a narrow range between 3 and 4 , this term is usually negligible.

To reduce the computation time associated with field and energy calculations (steps 2 and 4 in Fig. 3.4), the background $\tilde{\mathcal{B}}^{*}$ can be electrostatically coarse-grained, with molecular fragments or molecules as a whole replaced by a single interaction site. The reciprocal-space calculation in particular will hardly lose in accuracy upon such a coarse-graining step. In spherical-tensor notation, the moments of the coarse-grained site at position $\boldsymbol{r}_{c g}$ relate to the atomic contributions at positions $\left\{\boldsymbol{r}_{d}\right\}$ via [110]

$$
\begin{aligned}
Q_{n m}\left[\boldsymbol{r}_{c g}\right] & =\sum_{d \in D} \sum_{l=0}^{L} \sum_{k=-l}^{l}\left[\left(\begin{array}{c}
n+m \\
l+k
\end{array}\right)\left(\begin{array}{c}
n-m \\
l-k
\end{array}\right)\right]^{1 / 2} . \\
& \cdot R_{n-l, m-k}\left(\boldsymbol{r}_{c g}-\boldsymbol{r}_{d}\right) \cdot Q_{l k}\left[\boldsymbol{r}_{d}\right]
\end{aligned}
$$

where $R$ denotes a regular spherical harmonic. Note that the above equation, routinely used in fast-multipole methods, holds for the complex multipole moments $Q_{n m}$. These are related 


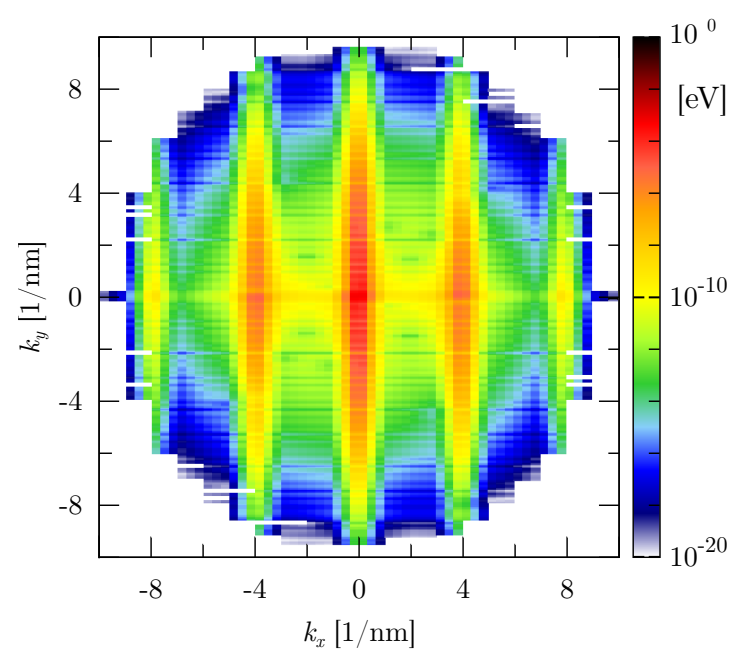

Figure 3.5. Reciprocal-space resonances in structurally ordered morphologies. Root-mean-square energy contribution map (in $\mathrm{eV}$ ) of reciprocal-space vectors $\boldsymbol{k}=\left(k_{x}, k_{y}, 0\right)$ in the plane of a $\mathrm{C}_{60} / \mathrm{D} 5 \mathrm{M}$ interface. Here, the pronounced resonances arise from patching of the respective crystal structures required to reduce the interfacial mismatch in an $x y$-periodic description. Similar resonances should, however, be expected for any preferentially ordered system.

to their real counterparts (for which the interaction tensors $T_{t u}^{a b}$ in Eq. 3.33 were defined) via

$$
\left(\begin{array}{l}
Q_{l+m} \\
Q_{l-m}
\end{array}\right)=\frac{1}{\sqrt{2}}\left(\begin{array}{cc}
(-1)^{m} & i(-1)^{m} \\
1 & -i
\end{array}\right)\left(\begin{array}{l}
Q_{l m c} \\
Q_{l m s}
\end{array}\right) .
$$

Finally, the precomputation of structure factors $\mathcal{S}\left(\boldsymbol{k} ; \mathcal{B}^{*}\right)$, to be reused for each site calculation, is a second way how to speed up field and energy computations in reciprocal space. In systems with pronounced nematic or biaxial ordering, reciprocal-space resonances (see Fig. 3.5) may appear in the structure factors, such that a summation over spherical $k$-shells may terminate prematurely. Rating functions for $k$-vector contributions have proven helpful in achieving a faster convergence of the $k$-space sum, if the order directors are aligned with one of the system axes:

$$
\begin{aligned}
& g_{3}(\boldsymbol{k})=\frac{\mathcal{S}\left(k_{x} \hat{e}_{x}\right) \mathcal{S}\left(k_{y} \hat{e}_{y}\right) \mathcal{S}\left(k_{z} \hat{e}_{z}\right)}{\left(\left\langle\mathcal{S}\left(k_{x}^{\prime} \hat{e}_{x}\right)\right\rangle_{x}\left\langle\mathcal{S}\left(k_{y}^{\prime} \hat{e}_{y}\right)\right\rangle_{y}\left\langle\mathcal{S}\left(k_{z}^{\prime} \hat{e}_{z}\right)\right\rangle_{z}\right)^{2 / 3}} \mathcal{A}(k), \\
& g_{2}(\boldsymbol{k})=\frac{\mathcal{S}\left(k_{i} \hat{e}_{i}\right) \mathcal{S}\left(k_{j} \hat{e}_{j}\right)}{\left(\left\langle\mathcal{S}\left(k_{i}^{\prime} \hat{e}_{i}\right)\right\rangle_{i}\left\langle\mathcal{S}\left(k_{j}^{\prime} \hat{e}_{j}\right)\right\rangle_{j}\right)^{2 / 3}} \mathcal{A}(k) .
\end{aligned}
$$

These functions serve as a metric to sort $k$-vectors into summation shells. The ratings incorporate structure factors $\mathcal{S}\left(k_{i} \hat{e}_{i}\right)$ evaluated along the system axes $\hat{e}_{x}, \hat{e}_{y}$ and $\hat{e}_{z}$ - i.e., they 
take into account scan lines in $k$-space along the three primary axes, and from these scan lines construct a rating for off-axis $\boldsymbol{k}$-vectors. Accordingly, $g_{3}$ is the rating function used for off-axis $k$-vectors with three non-zero components, $g_{2}$ is the rating function for in-plane $k$-vectors with two non-zero components. The convergence criterion for off-axis and in-plane $k$-vectors is chosen as the root-mean square contribution of $k$-shells to fields and energies associated with the polarization cloud $\mathcal{P}^{(s)}$.

\subsection{Summary: Long-Range Polarized Embedding}

The long-range polarized embedding approach presented in this chapter targets the energy landscape of localized electronic states in a variety of systems encountered in the context of organic electronic devices. The technique is built on a perturbative approach in a classical expansion. Implemented in the VOTCA package [50], it is readily parametrized from first principles, taking into account both polarization and electrostatics with higher-order atomic multipoles. In particular, it successfully copes with the slowly-convergent charge-quadrupole interaction that typifies many molecular conjugated materials.

Next to advanced polarization approaches that capture charge flow [102], a promising extension of the technique focuses on the description of metal/organic interfaces, as they frequently appear in electronic devices. This should include both an explicit simulation of the interaction with the electrode [126], as well as charge equilibration effects across the interface [127]. Finally, the embedding procedure can also be interfaced with a quantummechanically treated region in cases where a quantum description of the electronic state proves essential. 


\section{Chapter 4}

\section{Charge Carriers at Organic- ORGANIC INTERFACES}

Structural order in organic semiconductors is paramount: It reduces energetic disorder, boosts charge and exciton mobilities, and in solar cells assists exciton splitting. Due to spatial localization of electronic states, microscopic descriptions of elementary processes tend to overlook the influence of structural features at a mesoscale. Long-range electrostatic interactions nevertheless probe this ordering, making local properties depend on the mesoscopic order. Using the computational techniques developed in Chapter 3, we show how inclusion of mesoscale order resolves the controversy between experimental and theoretical results for the energy-level profile and alignment in a variety of organic systems, with direct experimental validation. Optimal use of long-range ordering also rationalizes the acceptor-donor-acceptor paradigm for the molecular design of donor dyes in organic solar cells. We calculate the charge-densitydependent open-circuit voltage across planar heterojunctions in excellent agreement with experimental data, based only on crystal structures and interfacial orientation.

\subsection{The Acceptor-Donor-Acceptor Puzzle}

As outlined in Chapter 1, the performance of organic solar cells relies on the sequential conversion of a photon into free charges via light absorption, then exciton diffusion and dissociation, and finally charge drift-diffusion and extraction [129-131]. The efficiency of these processes can be optimized by fine-tuning the underlying molecular structures. Design rules targeting enhanced exciton and charge diffusion or exciton splitting are, however, hard to come by, since the underlying microscopic mechanisms are not well understood. Efficient exciton dissociation, for example, has been attributed to the assistance of charge separation by a gradient in the free-energy landscape $[132,133]$, structural heterogeneity as a function of distance to the interface [134], doping and charged defects [135], increase in entropy as the electron and hole move away from the interface [136], formation of hot CT states [137], or long-range tunneling [138]. 


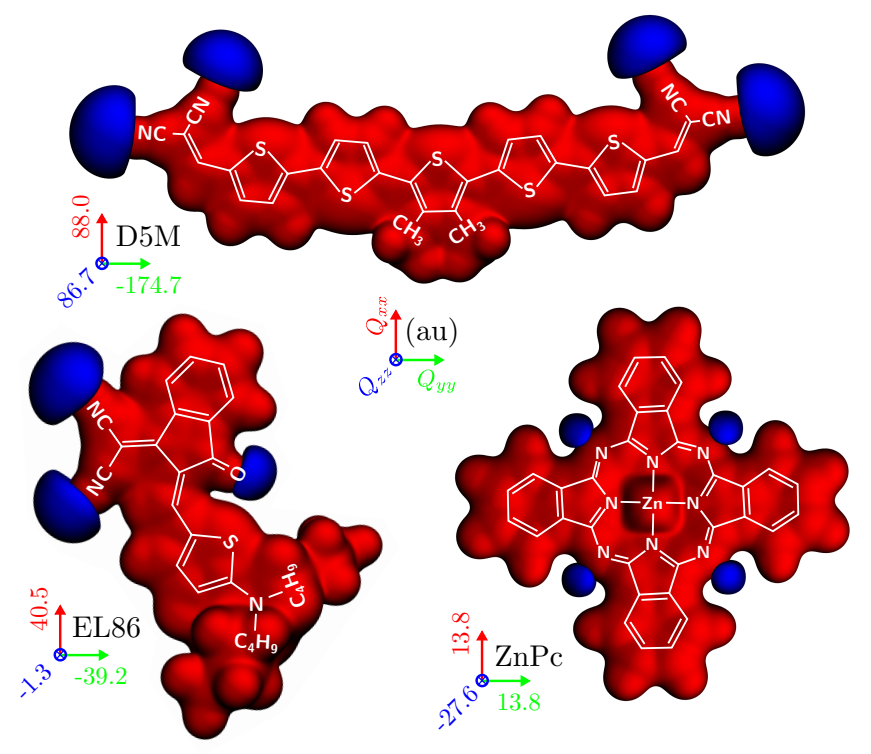

Figure 4.1. Electrostatic potential of donor materials. Isosurfaces (blue $-1 \mathrm{~V}$, red $+1 \mathrm{~V}$ ) and chemical structures of D5M [9] (ADA architecture), EL86 [128] (DA architecture) and $\mathrm{ZnPc}$. Quadrupole moments are given in atomic units along all three principal axes, as indicated.

Tuning optical absorption profiles, by contrast, is a more manageable approach to enhance the external quantum efficiency of single-junction devices. Here, we discuss the acceptordonor-acceptor (ADA) molecular architecture [30-32, 139] - a typical molecular design pattern targeting the absorption strength of an organic dye. The ADA pattern has been successfully employed in the family of dicyanovinyl-substituted oligothiophenes (DCVnTs) [32]: The methylated derivative DCV5T-Me(3,3) (D5M, see Fig. 4.1) is world-record holder among evaporated small-molecular donor materials with disclosed chemical structure, yielding a certified power-conversion efficiency of 8.3\% [26] (see Fig. 1.2 for the device stack). Since this high efficiency cannot be explained solely on the basis of enhanced absorption profiles, microscopic insight offered by a variety of computational techniques is desirable [50, 133, 140-142].

However - theoretical studies of several of the most efficient systems, DCVnT/ $\mathrm{C}_{60}$ in particular, predict energy levels that should render the solar cell dysfunctional: Neither level bending nor level offset obtained at the donor-acceptor interface promote splitting of geminate hole-electron pairs [143]. On the level of charge energetics, the reason for the exceptional performance of this class of materials and more generally the ADA architecture simply eludes rationale. The pronounced negative long-axis quadrupole component that is distinctive of ADA-type materials (Fig. 4.1) only aggravates what is already problematic on a gas-phase level, namely, the vacuum electron affinity (EA) of the supposed donor D5M falling below the EA of $\mathrm{C}_{60}$ [143]. As this puzzle is a matter of interface energetics, it connects with a number of studies that establish links among interface structure, level bending and offset $[97,99,100$, 


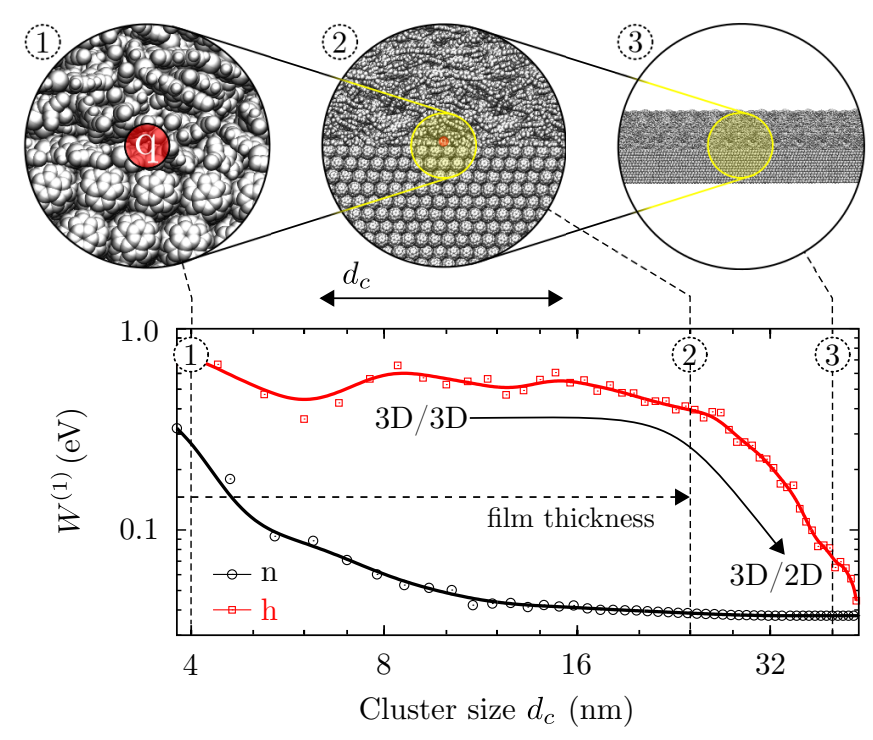

Figure 4.2. Convergence of energy levels. Variation of the electrostatic interaction energy $W^{(1)}$ with cluster diameter $d_{c}$ for a positively charged ( $h$, squares) and neutral $(n$, circles) DCV4T molecule in a nematically ordered thin film of thickness $12 \mathrm{~nm}$, with solid lines as guide to the eye. Insets 1-3 illustrate the cluster shape and scale at the respective $d_{c}$ as indicated, with shell-by-shell growth around the central molecular ion $q$. The crossover from a bulk-like to a slab-like convergence characteristic occurs as $d_{c} / 2$ exceeds the film thickness.

140, 144-146]. It turns out that methods used therein cannot account for the performance of $\mathrm{D} 5 \mathrm{M} / \mathrm{C}_{60}$ either. At the same time, there is no reason to believe that this material system is exceptional from a physical point of view. Rather, inconsistencies revealed for D5M/C 60 should also afflict other donor/acceptor combinations.

In this chapter, we address these inconsistencies, providing the link between molecular structure, energy landscape of the donor/acceptor interface and open-circuit voltage for various donor architectures, starting with the ADA pattern. We show that the energy landscape is shaped by mesoscale structural order on a length scale in excess of $100 \mathrm{~nm}$ and that the associated long-range electrostatic effects not only resolve existing inconsistencies, but also rationalize the high efficiency of $\mathrm{D} 5 \mathrm{M} / \mathrm{C}_{60}$.

Our analysis will be based on the simulation approach detailed in Chapter 3. Here, we only recapitulate its main features: It is based on a perturbative description of localized excitations (here charges) in their molecular surrounding. The excitation energy is decomposed into an intra- and intermolecular part. The former is given by the gas-phase electron affinity (EA) for electrons $(e)$ or ionization energy (IE) for holes $(h)$. The latter consists of the electrostatic (1st-order) and induction (2nd-order) corrections $\Delta_{s}^{(1)}$ and $\Delta_{s}^{(2)}$, where $\Delta_{s}^{(i)} \equiv W_{s}^{(i)}-W_{n}^{(i)}$ with charge state $s \in\{e, h\}$ is calculated with respect to the perturbative contribution $W_{n}^{(i)}$ to the neutral $(s=n)$ ground state.

The essence of the computational framework is an appropriate treatment of the longranged charge-quadrupole interaction on a mesoscale. In organic semiconductors, the lead- 
ing multipole moment in the expansion of the charge density originates from the molecular quadrupole. The charge-quadrupole interaction energy scales as $r^{-3}$ with distance $r$. Therefore, the energy of a molecular ion embedded in a 3D-infinite bulk environment is conditionally convergent, i.e., can in principle assume any value depending on the surface structure [147]. The situation is mitigated in lower-dimensional systems. Still, in particular in 2D-infinite thin films, the energy convergence is not only slow, but for cluster sizes smaller than the thin-film thickness also passes through a deceptive plateau that resembles the bulk, rather than thin-film, energetics. This is illustrated in Fig. 4.2, where the electrostatic energies for a positively charged and a neutral molecule embedded in an evaporated (see Sec. 2.2) $12 \mathrm{~nm}$ thick slab of $\mathrm{DCV} 4 \mathrm{~T} / \mathrm{C}_{60}$ are shown as a function of the cluster size $d_{c}$. For a hole, this energy contribution is far from converged even for $d_{c}=40 \mathrm{~nm}$ (as was already illustrated for perfect crystals in Fig. 3.1). However, with a typical cutoff of $4-8 \mathrm{~nm}$ used in simulations, one might interpret the plateau in the $8-20 \mathrm{~nm}$ range $(0.5 \mathrm{eV})$ as the site energy of a hole at the interface, whereas the actual converged value for the $2 \mathrm{D}$-infinite system is $-0.7 \mathrm{eV}$.

Even though electrostatic effects in organic semiconductors have been linked to the molecular quadrupole in a variety of theoretical and experimental studies [97, 99, 100, 104, 105, 140, 144-146, 148], the routine use of an interaction range cutoff resulted in a very different and significantly more local interpretation of electrostatic effects, as will be illustrated in the following section.

\subsubsection{Level Profiles Across $\mathrm{C}_{60} / \mathrm{D} 5 \mathrm{M}$ Interfaces}

To illustrate how structural coherence fundamentally changes energy level profiles, we first compare systems with short- and long-range in-plane molecular order imposed at the $\mathrm{D} 5 \mathrm{M} / \mathrm{C}_{60}$ interface and subsequently generalize our results to other donor-acceptor heterojunctions.

The position-dependent electrostatic and induction corrections $\Delta^{(1,2)}$ to hole and electron site energies for the $\mathrm{D} 5 \mathrm{M} / \mathrm{C}_{60}$ system are shown in Fig. 4.3a-b. Use of a (sizeable) cutoff $r_{c}=6 \mathrm{~nm}$ for electrostatics and induction leads to pronounced level bending at the $\mathrm{C}_{60} / \mathrm{D} 5 \mathrm{M}$ $(z=0)$ and D5M/vacuum $(z=13 \mathrm{~nm})$ interfaces (Fig. 4.3a). The resulting level diagram (Fig. 4.3c) could give rise to a working solar cell - but only with the roles of donor and acceptor reversed, in clear contradiction to experiments. If we now account for long-range structural order $\left(r_{c}=\infty\right)$, the energy landscape changes entirely: Not only does the sign of the offset between donor and acceptor reverse, but the band bending that is present in Fig. 4.3a is levelled out, as shown in Fig. 4.3b. The change is mostly due to the electrostatic contribution $\Delta^{(1)}$. It is driven by the competition between in- and out-of-plane interactions that reflect the pronounced quadrupolar and steric anisotropy. Regarding level alignment and profile (Fig. 4.3d), this solar cell is functional again.

Polarization leads to a further increase of the donor-acceptor offset for hole and electron levels. This increase is due to the build-up of dipole layers across the interface. These have in fact been discussed to contribute to level bending [143, 146]. This conclusion applies, however, primarily to the short-range picture: With the long-range correction in place, as Fig. 4.3d 


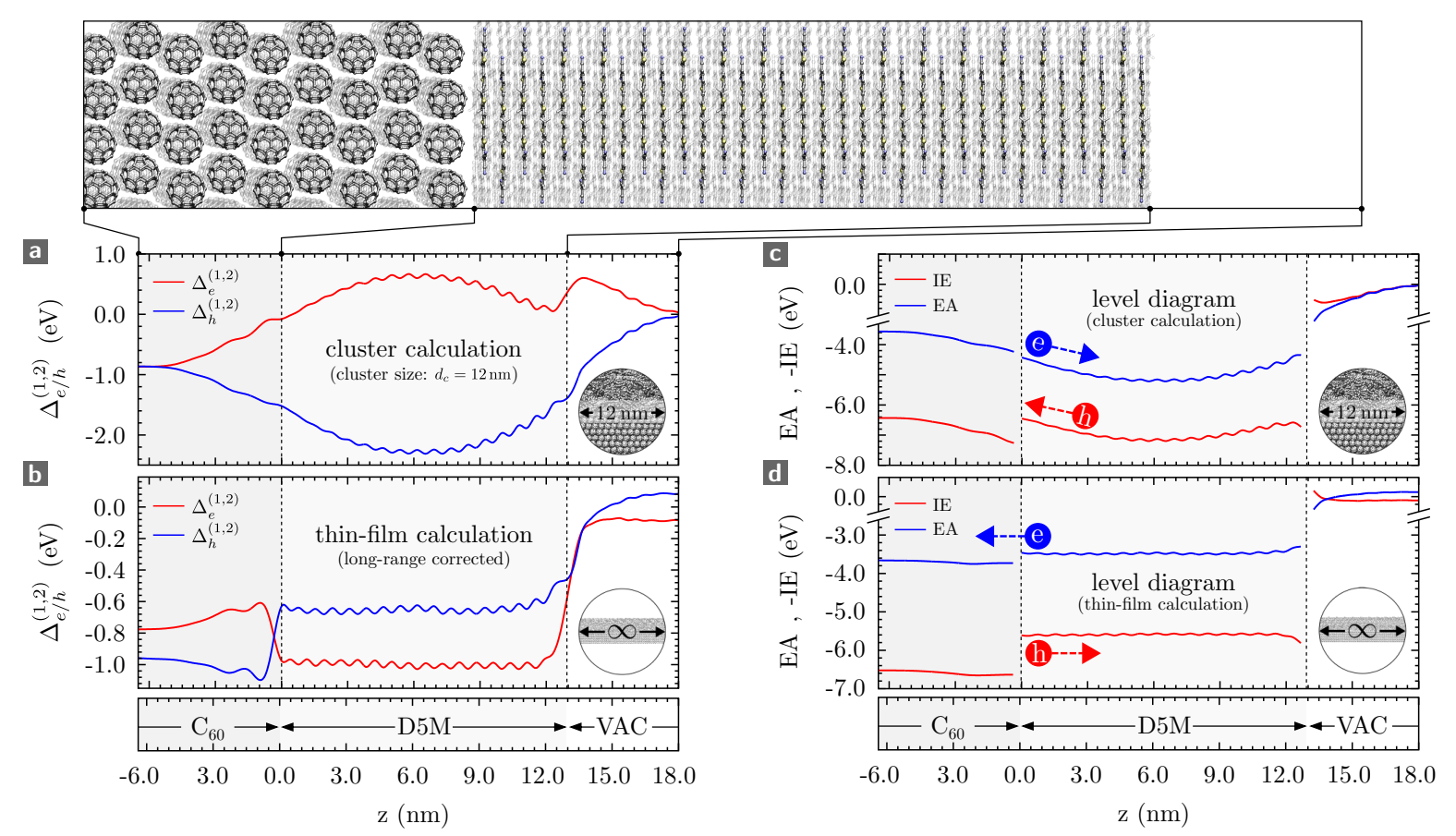

Figure 4.3. Hole and electron energy profiles with and without long-range correction. Position-dependent electrostatic and induction contribution $\Delta^{(1,2)}(z)$ to hole $(h)$ and electron $(e)$ site energies for a $\mathrm{C}_{60} / \mathrm{D} 5 \mathrm{M}$ crystal-crystal interface (a) in the semiconverged regime (cluster size $12 \mathrm{~nm}$ ) and (b) in the 2D-converged regime with infinite interaction range. The insets illustrate the system configuration used in the respective computation (spherical cluster or thin film). The level diagrams $(\mathrm{c}, \mathrm{d})$ for the two regimes differ qualitatively with respect to level bending and offset. The level alignment obtained for the $12 \mathrm{~nm}$ clusters (c) leads to trapping of holes at the interface and extraction of electrons towards the donor rather than acceptor. The long-range corrected scenario (d) provides flat energy profiles and offsets suited for a functional photovoltaic device (for illustration of gradients, blue circles represent electrons, red circles holes). Note that the cutoff procedure employed in (a) and (c) includes an electrostatic buffer shell which guards against parasitic polarization effects at the cluster edges. 


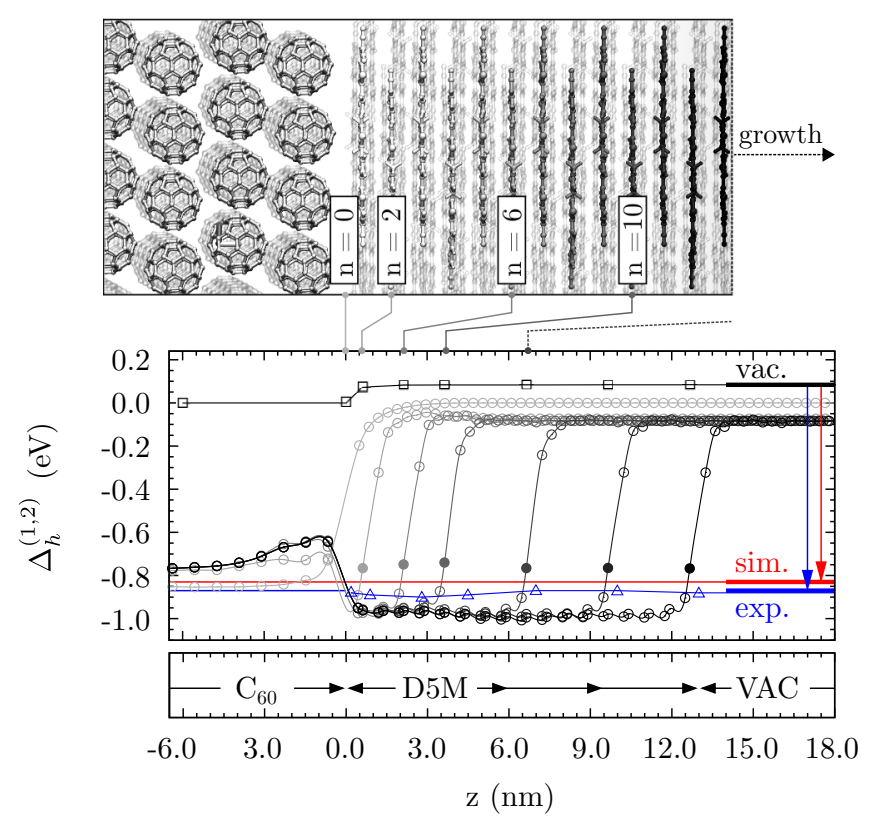

Figure 4.4. Evolution of energy profiles and offsets during growth of the donor layer. Energy profiles $\Delta_{h}^{(1,2)}(z)$ for holes (grey-scaled lines with circles) at various layer thicknesses $n$ of the donor (D5M) layer, ranging from $n=0$ (light grey) to $n=34$ (black) with intermediate steps at $n=2,6,10,18,26$. Solid circles indicate the topmost D5M monolayer. UPS values for $\Delta_{h}^{(1,2)}$ (blue solid line with triangles) have been calculated by subtracting the gas-phase IE from the measured potentials. In the comparison of the simulated average energy $\Delta_{h}^{(1,2)}$ (red horizontal line), the vacuum-level shift (line with squares) is taken into account.

shows, only the $\mathrm{C}_{60}$ levels remain slightly bent due to the interaction of the charge and its polarization cloud with a polarization layer in $\mathrm{C}_{60}$ in line with the step in quadrupolar fields. The donor IE and EA profiles are flat as a result of the large extension of the layer in the $x y$-plane.

Furthermore, the effect of surface quadrupoles has been described in terms of an effective dipole layer, as some of the quadrupolar tensorial components can be represented as two dipoles joined tail-to-tail [104, 105]. This approximation is, however, misguiding, as surface quadrupoles and dipoles promote different long-range electrostatics due to their distinct symmetry properties. Specifically, quadrupoles alone do not generate a shift of the vacuum level, only dipole layers do.

\subsubsection{Experimental Validation}

With the electrostatic and induction contributions taken into account, we can directly test our conclusions against experimental measurements performed by M. Tietze and C. Elschner (IAPP Dresden), who measured the ionization energy during stepwise growth of the donor 
D5M on a $\mathrm{C}_{60}$ substrate, see Fig. 4.4. The measurements, carried out via ultraviolet photoelectron spectroscopy (UPS), indicate a constant IE of $5.75 \mathrm{eV}$ for all thicknesses of the donor film. Simulations also produce a fast convergence to a single thin-film IE as of a film thickness of 1-2 nm (for the comparison, we assumed a penetration depth of two monolayers into the material). Correcting for the vacuum-level shift, which with the method at hand can be probed directly, as well as the internal gas-phase contribution $(6.63 \mathrm{eV})$, an excellent agreement for the D5M IE is achieved between experiment and theory (blue and red horizontal lines in Fig. 4.4). Both the qualitative and quantitative agreement indicate that electronic levels are indeed determined by long-range electrostatic effects that accompany mesoscale order and secure a flat energy profile within the domains.

\subsection{Orientation Effects and Film Patterning}

For the case of $\mathrm{C}_{60} / \mathrm{D} 5 \mathrm{M}$ presented above, we could already observe a qualitative impact of long-range interactions on energy level profiles. In this section, we will detail further implications that result from a long-range treatment in thin films of different orientation and patterning. To this end, we will for now abstain from the atomistic complexity of the previous section and instead apply the polarized embedding procedure to phenomenological lattice models.

First, to develop physical intuition and avoid important mechanisms being concealed by technicalities of the computational procedure, we start by investigating the relevant interaction sums in a qualitative way. We hence consider a cubic lattice of multipoles $Q_{l m}^{a(n)}=Q_{l m}$ with lattice spacing $c$, interfaced with an (in its ground state) apolar, polarizable medium. The electrostatic contribution $\Delta^{(1)}$ as a function of position $z$ of a charge $q$ measured along the interface normal can be written down in terms of lattice sums $\xi_{l m}(z / c)$,

$$
\Delta^{(1)}(z)=\sum_{l, m} q c^{-(l+1)} Q_{l m} \xi_{l m}(z / c)
$$

In an atomistic picture, the $Q_{l m}$ are determined by the molecular architecture, with the leading moment frequently given by the molecular quadrupole $(l=2)$ - the dipole vanishes due to either the inversion symmetry of the molecular ground state or dimerized unit cell. Furthermore, due to point symmetries of the lattice interface, only two of the five components of the quadrupole tensor are "interface-active" in the sense that they impact the level profile $\Delta^{(1)}(z)$ : These are $Q_{20}=Q_{z z}$ and $Q_{22 c} \sim\left(Q_{x x}-Q_{y y}\right)$. The associated electrostatic contribution reads

$$
\Delta_{l=2}^{(1)}(z)=q c^{-3}\left[Q_{20} \xi_{20}(z / c)+Q_{22 c} \xi_{22 c}(z / c)\right] .
$$

With the reduced lattice dimensions in direction $\hat{i} \equiv \hat{x}, \hat{j} \equiv \hat{y}$ and $\hat{k} \equiv \hat{z}$ given by $\tilde{a}=a / c$, 


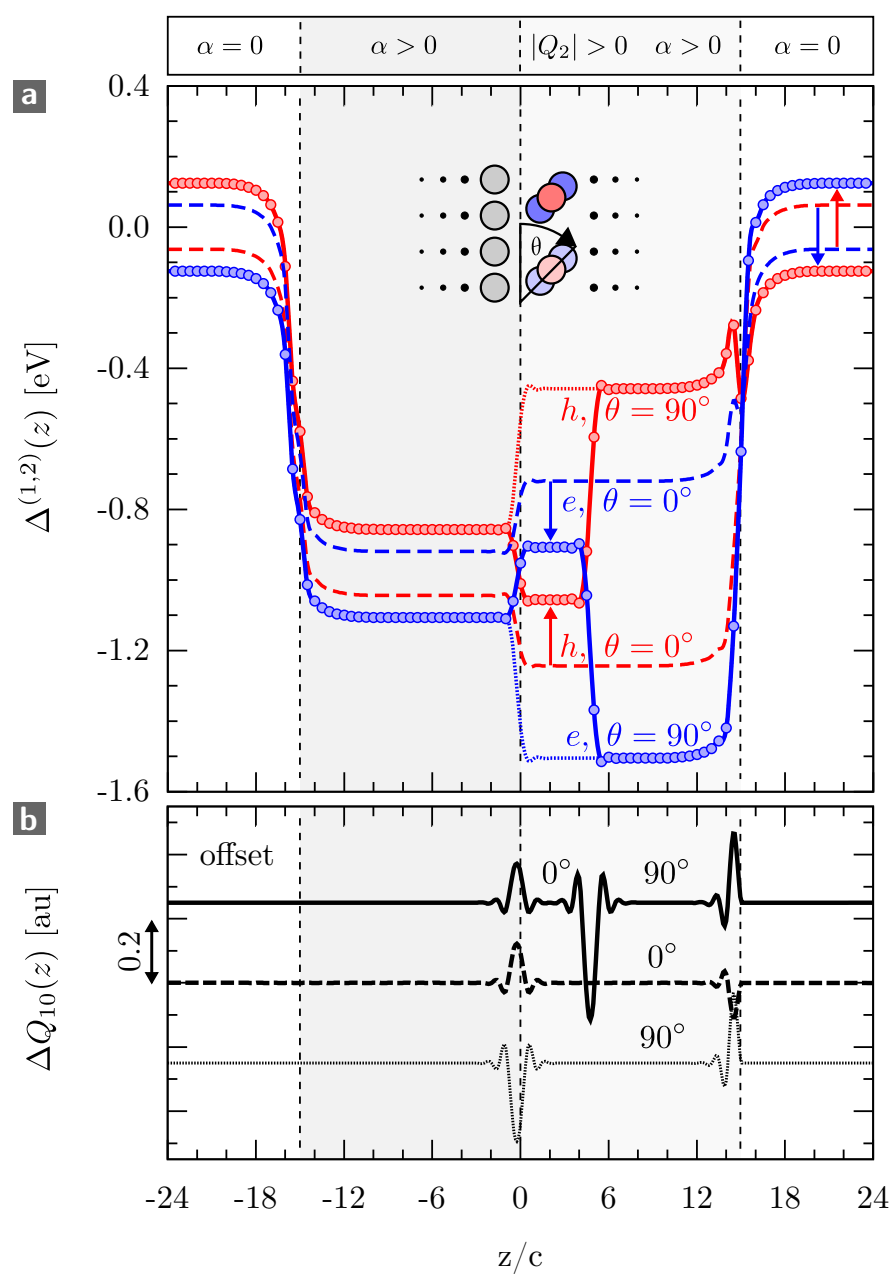

Figure 4.5. Lattice multilayers. (a) Level profile for holes (red) and electrons (blue) across a bcc lattice trilayer consisting of apolar $(z<0)$ and quadrupolar $\left(z>0, \tilde{Q}_{20}<0\right)$ polarizable lattice points with an orientation $\theta=0^{\circ}$ in the interlayer $(0<z<5 c)$ and $\theta=90^{\circ}$ in the toplayer. The dashed and dotted lines denote the level profiles obtained for bilayers with a donor orientation of $\theta=0^{\circ}$ and $\theta=90^{\circ}$, respectively. (b) Interfacial dipoles generated by quadrupolar fields for the bilayer (bottom and middle trace) and trilayer configurations (top trace). Additivity of the peaks indicates that vacuum-level alignment holds for the trilayer system, as can also be seen from direct comparison of the vacuum levels of holes and electrons and the relative positioning of the interlayer, see the arrows in (a). 
$\tilde{b}=b / c$ and $\tilde{c}=1$, respectively, the lattice interaction kernels are

$$
\begin{aligned}
& \xi_{20}^{(i j k)} \sim R_{i j k}^{-5}\left[k^{2}-\frac{1}{2}\left(\tilde{a}^{2} i^{2}+\tilde{b}^{2} j^{2}\right)\right], \\
& \xi_{22 c}^{(i j k)} \sim R_{i j k}^{-5}\left[\tilde{a}^{2} i^{2}-\tilde{b}^{2} j^{2}\right],
\end{aligned}
$$

where $R_{i j k}=\left(\tilde{a}^{2} i^{2}+\tilde{b}^{2} j^{2}+k^{2}\right)^{1 / 2}$. Notably, for a cubic lattice $(\tilde{a}=\tilde{b}=1), \xi_{22 c}=0$.

The symmetry of the interaction sum hence limits the relevant components to $Q_{20}$ and $Q_{22 c}$ - when, in fact, the molecular quadrupole tensor in its eigenbasis consists of the very same two components. In Eq. 4.2, however, $Q_{20}$ and $Q_{22 c}$ are defined with respect to the laboratory frame, with the substrate normal $\hat{n}=\hat{z}$ aligned with the $z$-axis. Starting from $\tilde{Q}_{20}$ and $\tilde{Q}_{22 c}$ as the quadrupolar eigenmoments of a molecule, the magnitudes of the corresponding components in the lab frame necessarily depend on the molecular orientation. With (active) Euler rotations $R\left(\Omega_{z y z}=\left\{0^{\circ}, \theta, 0^{\circ}\right\}\right)$, the $\theta$-dependence of $\tilde{Q}_{20}$ and $\tilde{Q}_{22 c}$ in the lab frame reads

$$
\begin{aligned}
Q_{20}(\theta) & =\frac{1}{2}\left(3 \cos ^{2} \theta-1\right) \tilde{Q}_{20}+\frac{\sqrt{3}}{2} \sin ^{2} \theta \tilde{Q}_{22 c}, \\
Q_{22 c}(\theta) & =\frac{\sqrt{3}}{2} \sin ^{2} \theta \tilde{Q}_{20}+\frac{1}{2}\left(1+\cos ^{2} \theta\right) \tilde{Q}_{22 c} .
\end{aligned}
$$

Beyond these straightforward rules that determine the prefactors in Eq. 4.2, long-range interactions are absorbed in the lattice sums, of which $\xi_{20}(z / c)$ and $\xi_{22 c}(z / c)$ with kernel $\xi_{2 m}^{i j k} \sim(z / c)^{-3}$ (Eqs. 4.3 and 4.4) are slowly convergent for slabs of finite thickness and conditionally convergent for infinite half-spaces, thus necessitating appropriate summation techniques as described in Sec. 3.3. The same necessity applies to off-lattice simulations of realistic morphologies, where compact expressions like the above are not available.

In addition to the electrostatic level profile $\Delta^{(1)}(z)$, the multipolar components $Q_{20}$ and $Q_{22 c}$ also drive the build-up of interfacial fields, which in turn generate interfacial dipoles $\Delta Q_{10}^{a}(z)$ as part of the polarization response of the lattice:

$$
\Delta Q_{10}^{a}(z)=\alpha_{1 z}^{a} c^{-4}\left[Q_{20} \eta_{20}(z / c)+Q_{22 c} \eta_{22 c}(z / c)\right] .
$$

The $\eta_{2 m}$ are lattice sums that peak at $z=0$, the position of the interface, and decay rapidly over the first few layers. The $\eta_{2 m}$ are of a more local character than the $\xi_{2 m}$, and furthermore exclude self-consistent polarization. Still, they provide a hint towards the role of polarization in the long-range picture, as will be discussed in the following section.

\subsubsection{Orientation Effects in Lattice Multilayers}

Keeping these qualitative considerations in mind, we will now study the level profiles of lattice multilayers using the long-range polarized embedding approach. We start with a bilayer composed of a $b c c$ lattice with lattice constant $c=0.7 \mathrm{~nm}$. Lattice sites in $z<0$ are populated with non-polar, sites in $z>0$ with quadrupolar polarizable particles. In the 
context of organic solar cells, the former could correspond to a fullerene $\left(\mathrm{C}_{60}\right)$ acceptor unit, the latter to a donor molecule with a finite quadrupole moment. Next to these molecular sites, we introduce vacuum probes on either side of the bilayer, which can be singly charged in order to calculate the vacuum levels of electrons and (fictitious) holes above the thin-film surfaces.

We swiftly address the parametrization of this lattice model. First, the polarizabilities of the two lattice species are matched, chosen such that they effect a dielectric constant $\varepsilon \simeq 3$. As opposed to the non-polar (acceptor) site, the polar (donor) site in $z>0$ carries a pure $\tilde{Q}_{20}<0$ quadrupole. The orientation of this site with respect to the substrate is measured by the angle $\theta$ between the substrate plane and the site's local $\hat{z}$-axis, along which $\tilde{Q}_{20}$ is defined (see inset of Fig. 4.5). $\tilde{Q}_{20}$ is chosen negative $(-10 \mathrm{au})$ as an abstraction of the electrostatic layout of typical donor units: For instance, aligning $\hat{z}$ with the long axis of an acceptor-donoracceptor-type compound would produce a negative $\tilde{Q}_{20}$, due to the larger electronegativity of the acceptor units. Similarly, aligning $\hat{z}$ with the normal of the molecular $\pi$-conjugated plane in an unsubstituted compound will also generate a negative $\tilde{Q}_{20}$, due to the net-positive nuclear plane which is sandwiched in between the negatively charged $\pi$-electron orbitals.

Fig. 4.5a shows the level profiles $\Delta^{(1,2)}(z)$ for hole and electron charge carriers across this bilayer. The dashed and dotted lines correspond to the $\theta=0^{\circ}$ (henceforth called faceon) and $\theta=90^{\circ}$ (tip-on) configuration, respectively. To interpret the profiles, we first note that the dielectric stabilization at this heterojunction would amount to almost $1 \mathrm{eV}$ in the absence of any polar species. Any deviation from this mean-field value is hence attributable to the action of the quadrupolar sites in $z>0$. Notably, the polarity of the interface changes from the face-on to the tip-on case. In the former scenario, holes are stronger stabilized in the donor part than the acceptor part, and vice versa for electrons. In the latter, tipon, scenario, the situation is reversed, with a two-fold increase in donor-acceptor offset that results from the $\theta$-dependence of the $Q_{20}$ component in the substrate frame: specifically, $Q_{20}\left(0^{\circ}\right)=-\frac{1}{2} Q_{20}\left(90^{\circ}\right)=-\frac{1}{2} \tilde{Q}_{20}$. Beyond the dielectric stabilization of charge carriers, the polarization response of the lattice sites to the abrupt step in quadrupolar fields generates interfacial dipole layers captured by Eq. 4.7. As can be seen in Fig. 4.5b, the dipole profile is sharply peaked across the interface, with a slight kickback in polarization density over the adjacent layers as a consequence of the $b c c$ packing. These dipole layers are responsible for the vacuum-level shift observed on both sides of the thin film (Fig. 4.5a).

What happens if we now sandwich a $0^{\circ}$ layer in between the non-polar substrate layer and a $90^{\circ}$ top layer? The resulting level profiles for electrons and holes (Fig. 4.5a, solid lines with dots) coincide with the $90^{\circ}$ profiles, except within the $z$-region of the interlayer, where it does not, however, match the $0^{\circ}$ trace: Instead, the profile experiences a shift that matches the change in vacuum level from the face-on to the tip-on configuration. This phenomenon, known as vacuum-level alignment $[149,150]$, can be rationalized by the additivity of interfacial dipole layers, where the dipole profile of the trilayer (top trace in Fig. 4.5b) can be constructed entirely from the profiles of the face-on and tip-on bilayers (bottom traces). 


\subsubsection{Level Profiles in Patterned Thin Films}

The level profiles obtained so far for lattice multilayers have reasonably straightforward characteristics: Quadrupolar fields generate abrupt steps in the energy profiles that feature contributions from both direct charge-quadrupole interactions and quadrupole-induced dipole layers. Within the films, profiles are flat. We have, however, already seen in Fig. 3.1b that level flatness should be seen as a consequence of long-range ordering in the layer. At the same time, we know from Eq. 4.2 that - at least on a cubic lattice - only the out-of-plane component of the quadrupole tensor impacts energy profiles.

In this section, we will show that an intuitive understanding may be significantly more difficult to come by once the systems turn more complex. To this end, we study both in-plane and out-of-plane level bending in a patterned thin film (see Fig. 4.6a). The polar sites are defined precisely as before, but are now arranged in a simple-cubic structure for simplicity, with a lattice constant $c=0.55 \mathrm{~nm}$. In the face-on and tip-on orientations, the contributions to hole energies then amount to $\Delta_{h}^{(1)}=-0.5 \mathrm{eV}$ and $1.0 \mathrm{eV}$, respectively. Here we have excluded polarization contributions; these values are therefore constant throughout the entire thin film, with only slight deviations for the bottom- and top-most layers.

If we now assemble a chequer-patterned thin film from these building blocks, the energy landscape changes fundamentally, as will be demonstrated for scan lines along $\hat{x}$ and $\hat{z}$, indicated in the left panel of Fig. 4.6a. In particular, strong level bending occurs both in the out-of-plane (Fig. 4.6b, left) and in-plane (Fig. 4.6b, right) directions. The columnar superstructure prevents the energy profiles to flatten out, as was still the case in Fig. 3.1b, as well as the lattice models from the previous section. One should hence be careful when stating that long-range order effects flat level profiles. Instead, it would be more accurate to conclude that homogeneous ordering with respect to the out-of-plane quadrupole component has this effect.

Still, it is in fact possible to recover flat profiles at least partially in the system. To this end, we set the spacing between the periodically repeated slabs to zero. As a trivial result of the resulting translational symmetry along the $\hat{z}$-direction, the $\mathrm{z}$-dependence is then removed, see Fig. 4.6c. Strikingly, the polarity at the centre of the columnar domains reverses (compare the right-hand side of Fig. 4.6b-c), due to the different interaction balance between the in-plane and out-of-plane directions.

Considering either of the two scenarios from Fig. 4.6b-c, one may have difficulties to imagine device operation in the energy landscape of these patterned films. Fortunately, crystallites in polycrystalline materials will not lead to in-plane interfaces of this character, since they exhibit preferential ordering on the substrate - with the result that the out-of-plane multipolar components are preserved. Still, it is interesting to note that the energy landscapes from Fig. 4.6b-c are in fact bounded by the electrostatic contributions in the pristine layers $(-0.5 \mathrm{eV}$ and $1.0 \mathrm{eV})$. This has consequences for device fabrication: If, for example, a device is built around a lowest energy configuration (such as the face-on structure of the $\tilde{Q}_{20}$ material provides for hole carriers) domain defects are less likely to create traps at domain-domain 

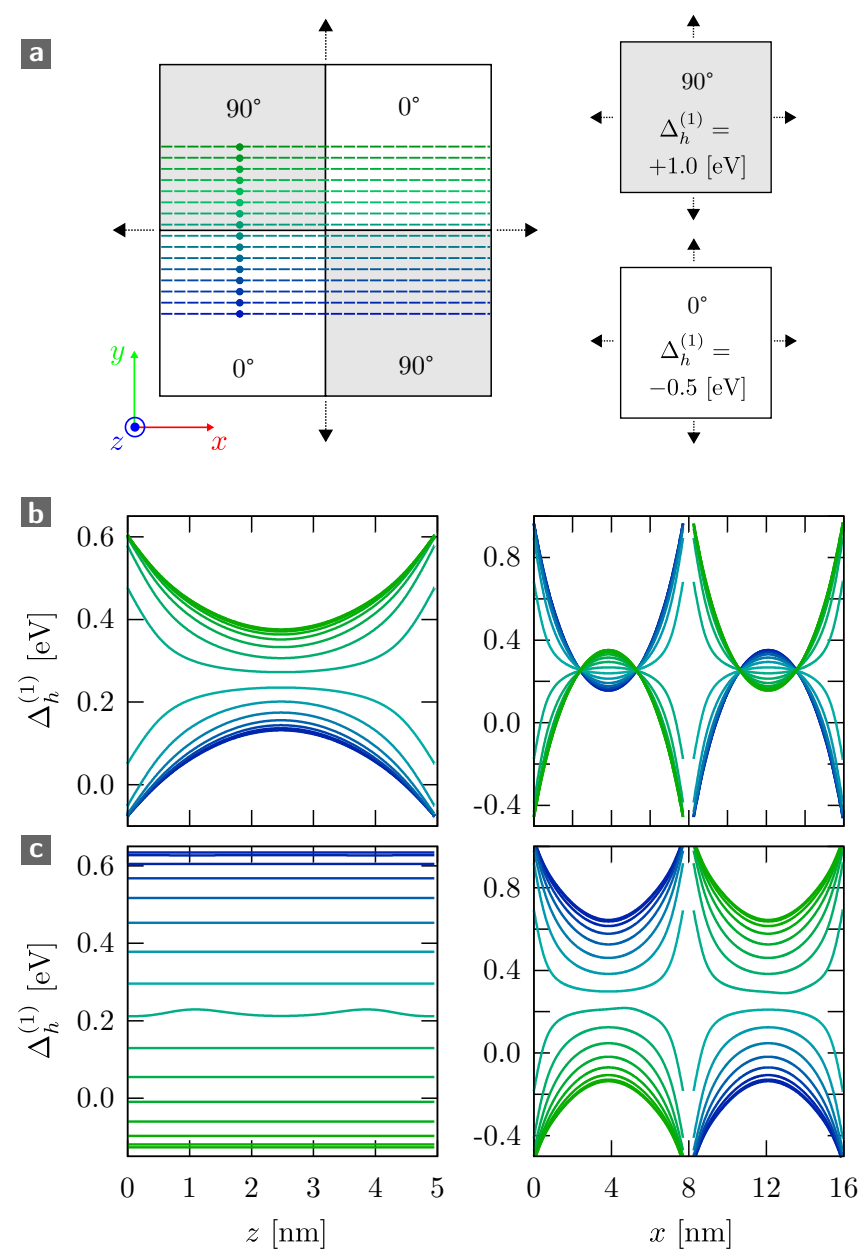

Figure 4.6. Patterned thin films. Chequerpatterned film (a, left) with $z$-aligned columns of $Q_{20}$-quadrupolar lattice points in an edge-on $(\theta=$ $\left.0^{\circ}\right)$ and tip-on $\left(\theta=90^{\circ}\right)$ orientation. Dots and dashed lines indicate scan lines along $z$ and $x$, respectively, as used for the $\Delta^{(1)}$ profiles $(\mathrm{b}, \mathrm{c})$. For pristine films (a, right), the electrostatic contribution is constant throughout the film, with values as indicated. $\Delta_{h}^{(1)}$ scan profiles for the chequerpatterned system from (a) were computed with a slab shape correction in a (b) repeated-slab setup with $10 \mathrm{~nm}$ spacing between films, and (c) with zero spacing between slabs, the latter corresponding to thick-film conditions. 
interfaces.

\subsection{Charge Carriers at Donor-Acceptor Heterojunctions}

In the previous section, we studied phenomenological lattice models to illustrate some of the effects of long-range interactions on the energy landscape of localized states in a polarizable, multipolar environment. The lattice sites in these models should be thought of as singlepoint expansions of entire molecules. However, due to the pronounced anisotropy of the conjugated molecules that typify organic semiconductors, such a lattice description in fact violates the criteria for a multipole expansion. In particular, the interaction distance between molecular expansion sites must be larger than the radii of the spheres that accommodate the electron density of the respective molecules. Systematic coarse-graining along Eq. 3.77 is therefore not an option in regions within which interactions between molecules need to be calculated explicitly. In the following, we will hence focus on atomistic descriptions of organic semiconductors in order to investigate the role of molecular orientation and packing (the latter being poorly captured on a lattice level) in a quantitative way.

\subsubsection{Interplay of Molecular Architecture, Packing and Orientation}

The physical mechanisms that shape the energy landscape are expected to apply to different donor/acceptor combinations in a generic fashion, as indicated by the lattice description from the previous section. Still, so far, we have only addressed the energetics at the $\mathrm{D} 5 \mathrm{M} / \mathrm{C}_{60}$ interface with atomistic resolution. It remains to be shown that the mesoscopic interaction range and quantitative scope of the method extend to other material systems. This extension will enable us to formulate structure-property relationships based on the interplay of molecular architecture, packing and orientation.

In total, we will compare among five donor materials used in organic solar cells (Fig. 4.8d): These include pentacene (PEN), sexithiophene (6T), zinc phthalocyanine ( $\mathrm{ZnPc})$, the merocyanine dye EL86 [128], and the acceptor-substituted oligothiophene D5M [9]. Of these materials, only EL86 and D5M have a ground-state dipole moment due to their donor-acceptor (DA) and cis-acceptor-donor-acceptor (ADA) architecture. The quadrupole tensors of PEN, $6 \mathrm{~T}$ and $\mathrm{ZnPc}$ are characterized by a negative out-of-plane and positive in-plane components, typical of hydrogenated $\pi$-conjugated molecules. D5M, by contrast, carries a sizeable negative long-axis quadrupole and a smaller positive out-of-plane component, as a result of its ADA structure. This distinctive quadrupolar layout has interesting implications for device operation [106], as will also become clear from the analysis in this section.

We will first show that these donor materials exhibit the same cluster-size dependence of energy levels that has already been exemplified in Fig. 4.2. For this purpose we consider thin films based on three different donor architectures (D, DA, ADA), with the molecular ion embedded in the centre of the film. The cluster-size and orientation-dependent contributions $W^{(1)}$ to the absolute state energies (neutral, hole and electron) are shown in Fig. 4.7a-c. As 


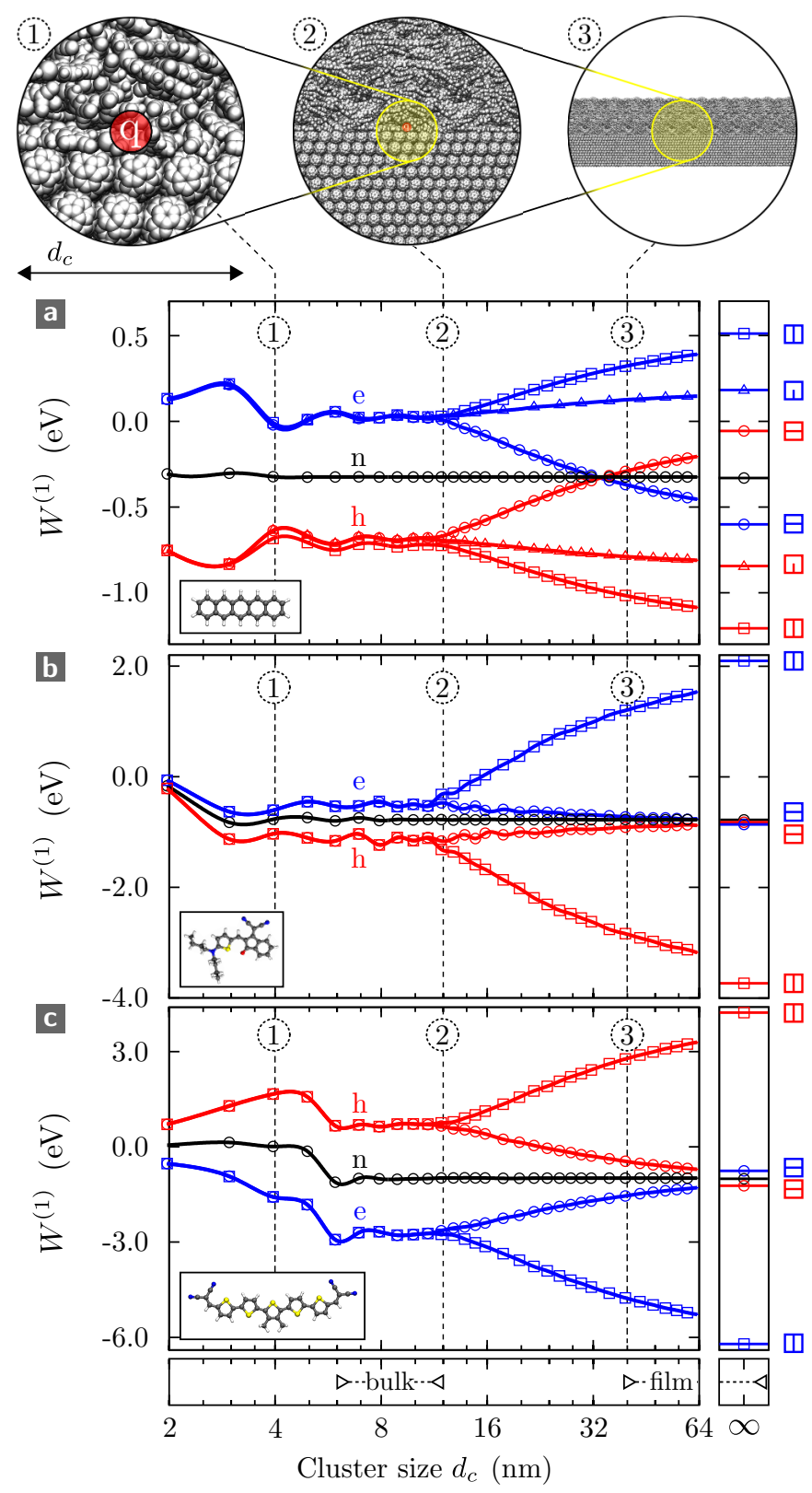

Figure 4.7. Orientation-dependent convergence trends for energy levels in pentacene, EL86 and D5M. Clustersize and orientation dependence of the electrostatic interaction energy $W^{(1)}$ in crystalline thin films of $10 \mathrm{~nm}$ thickness, composed of three different donor materials: pentacene (D architecture, a), EL86 (DA architecture, b) and D5M (ADA architecture, c). Black, blue and red lines pertain to $W^{(1)}\left(d_{c}\right)$ for the neutral, electron and hole state, respectively. Molecular orientation is indicated by circles (face-on), squares (short-edge-on) and triangles (long-edge-on), as furthermore represented by the cartoons in the rightmost panel. Insets 1-3 illustrate the cluster shape and scale at the respective $d_{c}$ as indicated, with shell-by-shell growth around the central molecular ion $q$. 
before, a semiconverged plateau that resembles bulk conditions is observed in the 6-12 nm range, which subsequently splits according to the orientation of the molecules in the thin film. The very slow convergence (which becomes yet slower with increasing film thickness) again hints at the electrostatic competition between in- and out-of-plane charge-quadrupole interactions. As a result, systems with moderate quadrupolar moments such as pentacene exhibit the same qualitative behaviour as the massively quadrupolar ADA compounds.

Even though most small-molecule-based materials for organic transistors and solar cells exhibit polycrystalline order, the simplest theoretical description starts from experimental unit cells, suited to study bulk energetics in these systems. As a first step in investigating the role of molecular architecture and packing, we therefore consider individual unit cells of the five materials in a 3D-periodic setup, thought to represent bulk conditions with well-defined surfaces. Fig. 4.8a summarizes the electrostatic and polarization contributions $\Delta^{(1)}$ and $\Delta^{(2)}$ for both electrons and holes, as well as the shape contribution $\Delta^{(*)}$ computed from Eq. 3.41. Note that the latter includes contributions from both electrostatics and polarization. First, it can be seen that the first-order correction $\Delta^{(1)}$ varies significantly among compounds, as does $\Delta^{(*)}$. For the same compound, asymmetric charging of the molecule (as in the case of EL86) may cause slight deviations from $\Delta_{h}^{(1)}=-\Delta_{e}^{(1)}$. Meanwhile, $\Delta^{(2)}$ is reasonably constant across different materials, ranging between $-0.9 \mathrm{eV}$ and $-1.0 \mathrm{eV}$. In strongly polar materials such as D5M with its high quadrupole density (leading to a sizeable shape contribution of more than $3 \mathrm{eV}$ ), deviations from this purely dielectric response may occur: A carrier-type dependent $\Delta^{(2)}$ results, for example, from the change in quadrupole moment upon charging, $\delta Q_{h} \neq \delta Q_{e}$. On a lattice level, an analytical estimate reads

$$
\Delta_{s}^{(2)}=-\frac{\alpha q^{2}}{2 c^{4}}\left[\sigma_{4}-\left(\frac{3 \delta Q_{20}}{q c^{2}}\right) \sigma_{6}+\frac{1}{4}\left(\frac{3 \delta Q_{20}}{q c^{2}}\right)^{2} \sigma_{8}\right] .
$$

This expression describes the effect of a non-zero $\delta Q_{20}$ (treated to first order in the induction loop) on the polarization contribution $\Delta^{(2)}$. Here, $\sigma_{4}$ to $\sigma_{8}$ are lattice sums which sensitively depend on molecular packing features, such as inter-level shift and lattice aspect ratio. Notably, both the $\sigma_{6^{-}}$and $\sigma_{8}$-term may significantly reduce (or enhance) polarization energies and hence necessitate an extension of the simple dielectric picture.

Fig. 4.8a seems to indicate that there are specific combinations of packing modes and molecular layouts, which energetically favour either holes or electrons. In fact, apart from $\Delta^{(*)}$, these exact same results could have also been extracted from a cutoff-based description, which - as seen in Fig. 3.1a - corresponds to the cubic/spherical limit (Eq. 3.41). Such an approach has been used in the past and indeed led to the conclusion that some packing modes (herringbone, brickwork) result in lower bulk hole energies than others [98]. A bulk description may, however, not be appropriate when simulating devices. Instead, a thin-film description becomes viable, where charge-carrier energies no longer depend on packing only, but also orientation - as illustrated by the lattice models from Sec. 4.2.1.

To explore the thin-film scenario, Fig. $4.8 \mathrm{~b}$ correlates the individual energy corrections to 

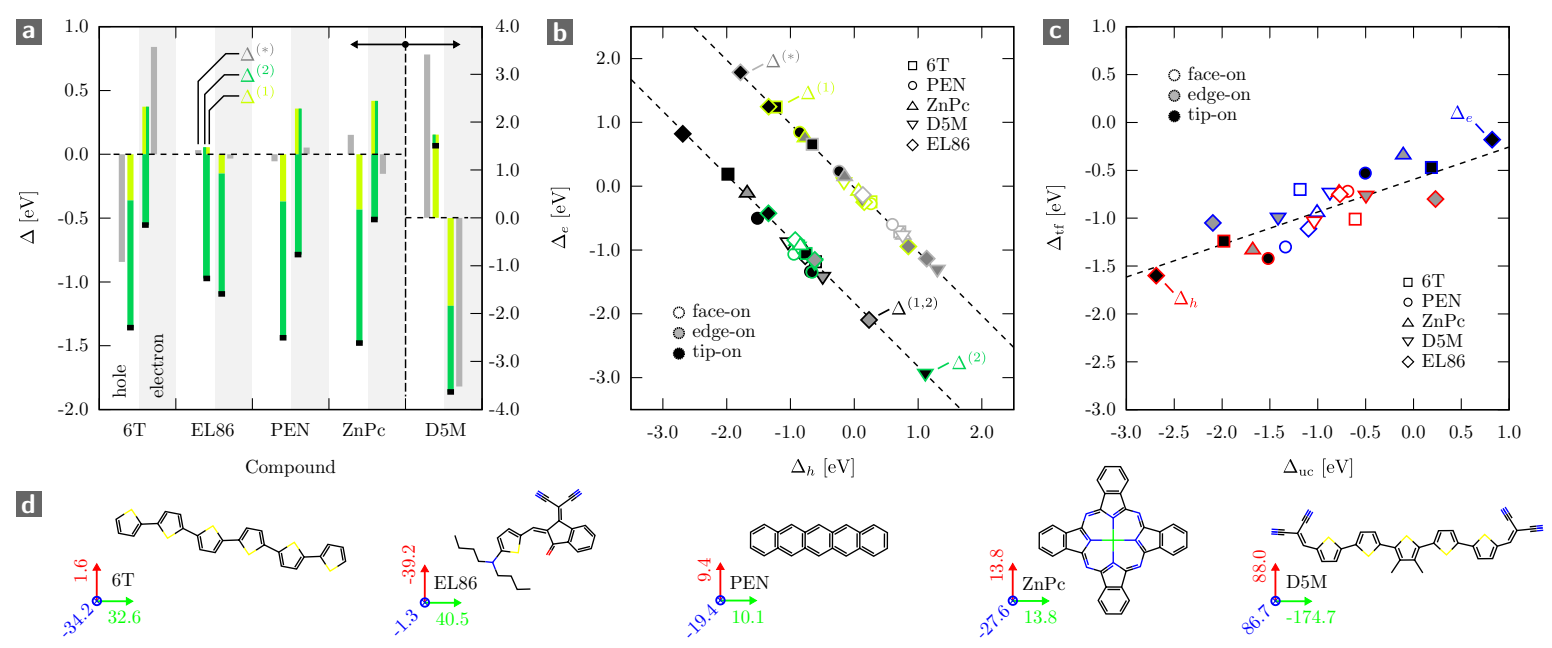

Figure 4.8. Comparison of bulk and thin-film energetics across different molecular materials. (a) Electrostatic $\left(\Delta^{(1)}\right.$, light green), polarization $\left(\Delta^{(2)}\right.$, dark green) and cube shape $\left(\Delta^{(*)}\right.$, grey) contributions to electron and hole levels computed from experimental unit cells. The black bars indicate the total solid-state contribution, $\Delta^{(1,2)}$. Note the different energy scale for D5M. (b) Correlation of electrostatic $\left(\Delta^{(1)}\right)$ and polarization $\left(\Delta^{(2)}\right)$ contributions to electron $\left(\Delta_{e}\right)$ and hole $\left(\Delta_{h}\right)$ site energies for thin-film levels calculated for differently oriented unit cells under application of the respective shape contribution $\left(\Delta^{(*)}\right.$, yellow). The symbol shape indicates the material $(6 \mathrm{~T}, \ldots, \mathrm{EL} 86)$, fill style the configuration (face-on, edge-on, tip-on). (c) Correlation between thin-film level contribution $\Delta_{\text {uc }}$ as computed from a single unit cell - see also panel (b) - versus the contribution $\Delta_{\text {tf }}$ calculated for a finite $10 \mathrm{~nm}$-thin film, for both holes (red) and electrons (blue). The definition of symbol shape and filling corresponds to the convention from panel (b). Chemical structures (d) are supplemented with the quadrupole components (in atomic units) along the eigenvectors of the quadrupole tensor. 
electron and hole levels $\left(\Delta^{(1)}, \Delta^{(2)}, \Delta^{(*)}\right.$ and $\left.\Delta^{(1,2)}\right)$ now calculated with a slab shape term (Eq. 3.42) applied along each of the three unit-cell vectors. The latter mimics different thinfilm orientations (face-on, edge-on, tip-on) as indicated by the fill style of the symbols. The symbol shape distinguishes between the five compounds. As becomes clear from a comparison of $\Delta^{(1)}$ (light-green symbols), general conclusions that link packing modes to electrostatic and polarization contributions are now impossible to formulate without at the same time accounting for molecular orientation. Specifically, orientations with $Q_{20}>0$ tend to produce a larger electrostatic stabilization of holes, as predicted by Eq. 3.42: For D5M, $Q_{20}>0$ is associated with a face-on, for $\mathrm{PEN}, 6 \mathrm{~T}$ and $\mathrm{ZnPc}$ with a tip-on orientation. Generally, the effect of orientation is sufficiently strong to produce both negative and positive $\Delta^{(1)}$ 's for the same carrier type, rendering molecular packing a not very useful descriptor in anticipating level positioning.

Note that the thin-film results from Fig. $4.8 \mathrm{~b}$ still pertain to simulations of 3D-periodic unit cells, where the interaction sums are conditionally convergent. In the context of organic electronic devices with their layered architecture, a 2D-periodic description becomes mandatory. As demonstrated in Fig. 3.3, such a 2D framework can be obtained effectively from a 3D-periodic description by introducing a vacuum buffer in between the periodically repeated slabs. Due to its convergence properties, the 2D framework simplifies the calculations conceptually; in particular, surface reconstruction is no longer required for a comparison with experiments. In Fig. 4.8c, the solid-state contributions that result for a $10 \mathrm{~nm}$ thin film of the respective material on top of $10 \mathrm{~nm} \mathrm{C}_{60}, \Delta_{\mathrm{tf}}$, are compared against the contributions $\Delta_{\mathrm{uc}}$ calculated from unit cells according to Fig. $4.8 \mathrm{~b}$. The strong correlation between $\Delta_{\text {tf }}$ and $\Delta_{\mathrm{uc}}$ indicates that both quantities are still determined by the same in-plane and out-of-plane multipolar components with, however, different prefactors, that originate in an absolutely vs. conditionally convergent interaction sum. Furthermore, interfacial effects (notably interface dipoles) impact $\Delta_{\mathrm{tf}}$, but remain absent in $\Delta_{\mathrm{uc}}$. A strict correlation should hence not be expected. A linear fit indicates a slope of $1 / 3$ of the correlation function (dashed line in Fig. 4.8c), which passes through the isopolar point at $\Delta_{\mathrm{tf}} \simeq \Delta_{\mathrm{uc}} \simeq-0.95 \mathrm{eV}$, where the system behaves like a pure dielectric composed of polarizable, non-polar building blocks. Fig. $4.8 \mathrm{c}$ therefore highlights that computationally inexpensive calculations performed on unit cells are already suited to estimate the energetics across thin films.

Still, an explicit account of the thin film as included by $\Delta_{\text {tf }}$ is required to enable a direct experimental validation of the simulation results. Taking into account the full energy landscape, we arrive at Fig. 4.9a, which compares calculated and measured thin-film IEs in a device setup. Drawing from different molecular design paradigms, the set of donor molecules covers a wide spectrum of gas-phase IEs, ranging between $5.2-6.8 \mathrm{eV}$. In order to address the surface sensitivity of UPS, simulated hole energies are shown as a function of the penetration depth $1 / \alpha$. It can be seen that the simulation results (blue bars) not only quantitatively reproduce measured trends for the orientation dependence, but also the absolute experimental IE values extracted from UPS (black bars). The largest deviation occurs for pentacene, where UPS measures a larger stabilization than is simulated; this could be due to the enhanced hole 

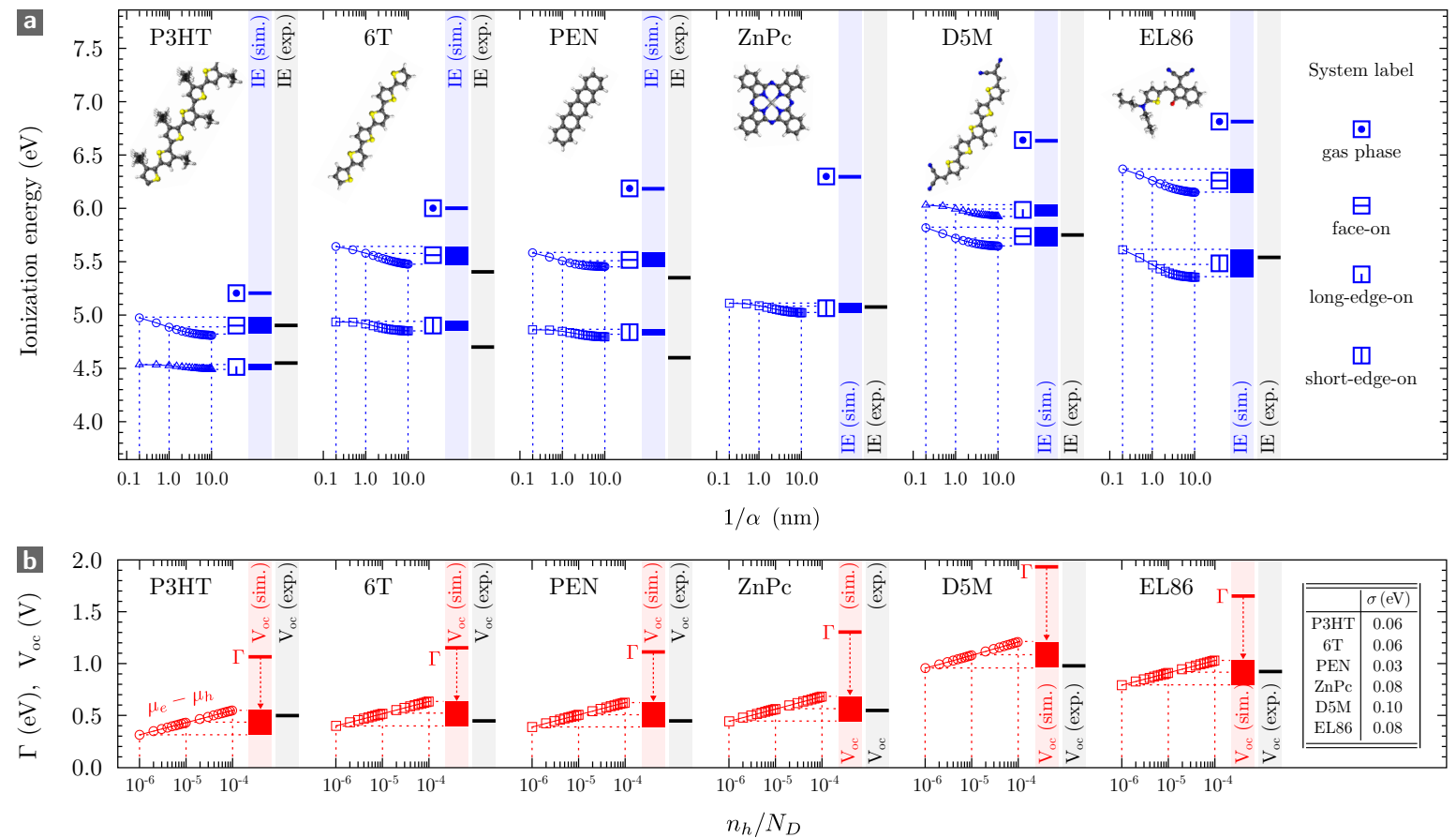

Figure 4.9. Ionization energies and open-circuit voltage across different donoracceptor interfaces. (a) Simulated (blue) and experimental (black) ionization energies of different donor materials with molecular structures as shown in the inset. The simulated $1 / \alpha$ sampling-depth dependence (blue curves, with dashed lines as guide to the eye) quantifies the reduced dielectric stabilization at the donor-vacuum interface as compared to the donoracceptor interface, to be taken into account in the comparison with the surface-sensitive UPS technique. Experimental IEs for P3HT, 6T, PEN and ZnPc are taken from Refs. [104, 151154]. Gas-phase IEs (blue) were calculated via B3LYP/6-311+g(d,p). (b) Simulated photovoltaic gap $\Gamma$ and charge-density-dependent open-circuit voltage $V_{\text {oc }}$ (red curves and bars) of the planar heterojunction compared to the experimental $V_{\text {oc }}$ (black bars). Line symbols correspond to the configurations from (a). The calculation of the difference in chemical potential used simulated disorder strengths $\sigma$ as listed in the inset. The charge-density dependence is expressed in terms of the occupation fraction $p=n_{h} / N_{D}$ of donor states. Experimental values for $V_{\text {oc }}$ for P3HT, 6T, PEN and ZnPc are taken from Refs. [152, 154, 155]. All simulated and experimental $V_{\mathrm{oc}}$ values apply to the respective planar heterojunction. 
delocalization that accompanies the very high charge mobility observed in this compound. Still, even then the molecular orientation can be deduced from a single IE measurement, without laborious structural characterization.

\subsubsection{The Charge-Density-Dependent Open-Circuit Voltage}

We continue with a full characterization of the single-carrier energy landscape of planar organic/organic heterojunctions represented by a level diagram such as shown in Fig. 4.10 for $\mathrm{C}_{60} / \mathrm{D} 5 \mathrm{M}$. This diagram again highlights the flat level profiles for ionization energies and electron affinities, which give rise to the well-defined thin-film contribution $\Delta_{\mathrm{tf}}$ analysed above. As an important extension to Fig. 4.3, the diagram furthermore indicates the broadening of the D5M density of states (DOS) calculated from supercells of the bulk material. This finite width results statistically from the evaluation of site energies of thousands of molecules, rendered possible by the low computational cost of the perturbative treatment.

The broadening of the DOS is caused by thermal disorder, which impacts the energetics even in highly crystalline materials [156]. Here, we either simulate thermal effects via molecular dynamics simulations (compare also Fig. 2.5) or estimate it from the compound's paracrystallinity. In all cases, the resulting distributions of hole energies are approximately Gaussian, with widths $\sigma$ on the order of $30-100 \mathrm{meV}$ obtained for the donor materials studied here (see the inset in Fig. 4.9b). Assuming sufficiently fast relaxation of charge carriers, this disorder, together with the hole charge density determine both the transport level located approximately $-\sigma^{2} / k_{\mathrm{B}} T$ below the mean of the DOS, and the chemical potential $\mu_{h}$ in the donor layer. The charge-density dependent (open-circuit) voltage across the organic/organic interface follows from the difference in chemical potential (quasi Fermi level) of holes $\left(\mu_{h}\right)$ and electrons $\left(\mu_{e}\right)$ on the donor and acceptor side [157]. For holes, the relationship between chemical potential $\mu_{h}$ and charge density, expressed in terms of the occupation fraction $p$ of hole-carrier sites, reads

$$
p=\frac{\int g_{D h}\left(\epsilon ; \sigma_{D h}\right) f_{h}\left(\epsilon ; \mu_{h}\right) \mathrm{d} \epsilon}{\int g_{D h}\left(\epsilon ; \sigma_{D h}\right) \mathrm{d} \epsilon} .
$$

Here, $g_{D h}\left(\epsilon ; \sigma_{D h}\right)$ denotes the hole DOS of the donor with width $\sigma_{D h}, f_{h}\left(\epsilon ; \mu_{h}\right)$ is the FermiDirac distribution. With a target charge density $p^{*}$, Eq. 4.8 can be solved iteratively for $\mu_{h}$ with an update function $\Delta \mu=-\sigma \log \left(p / p^{*}\right)$. For electrons (which reside on acceptor sites) analogous equations hold. In the voltage calculation, a charge-neutrality criterion, $n N_{A}=p N_{D}$, should furthermore be observed, where $N_{D}\left(N_{A}\right)$ denotes the number of donor (acceptor) sites in the system.

Open-circuit voltages obtained in this way are summarized in Fig. 4.9b. The comparison between the computed (red bars) and measured (black bars) voltages displays excellent agreement within the range of typical charge densities under open-circuit conditions and AM1.5g illumination $\left(p=10^{-5}\right.$, corresponding to a number density of $\simeq 10^{16} \mathrm{~cm}^{-3}$ ). The agreement once more indicates that the effective 2D-periodic description employed for $\Delta_{\mathrm{tf}}$ serves as a 


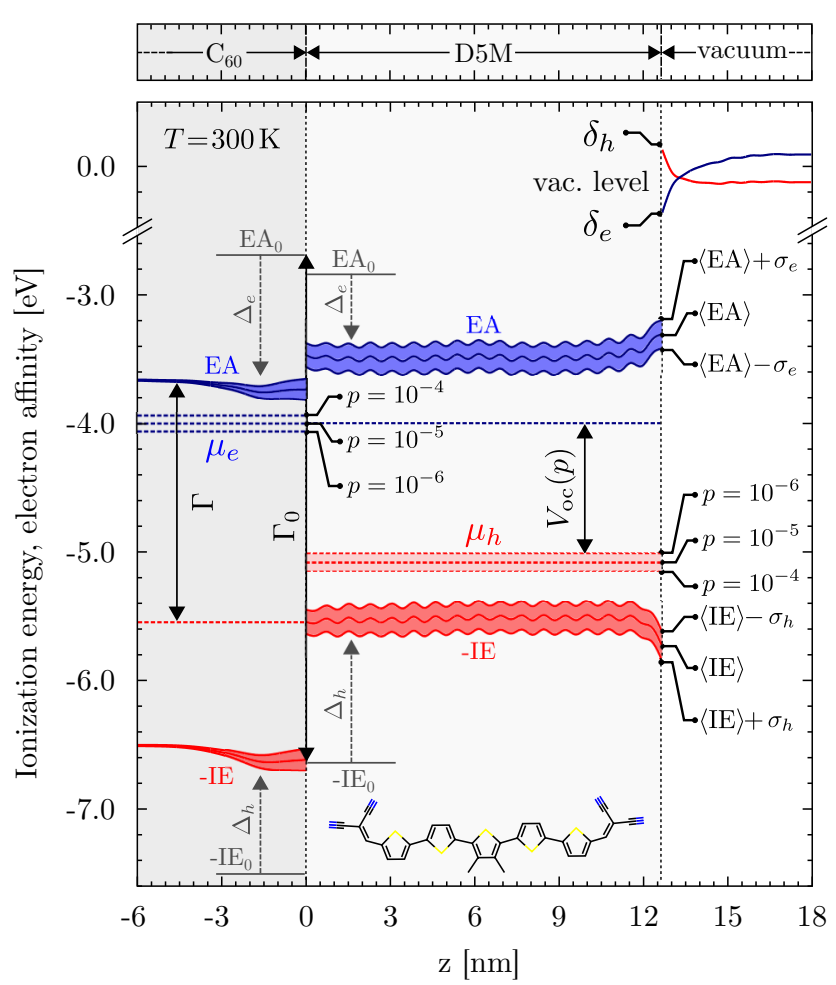

Figure 4.10. Single-carrier level diagram for $\mathrm{C}_{60} / \mathrm{D} 5 \mathrm{M}$. Electron affinity and ionization energy profiles across a $\mathrm{C}_{60} / \mathrm{D} 5 \mathrm{M}$ heterojunction, with positional broadening for $\mathrm{C}_{60}$ and thermal broadening (extracted from larger supercells) for D5M $\left(\sigma_{e}, \sigma_{h}\right)$ as indicated. Furthermore shown are the gas-phase levels $\left(\mathrm{IE}_{0}\right.$ and $\mathrm{EA}_{0}$ ) for the acceptor and donor species. The charge-densitydependent open-circuit voltage follows from the difference in quasi Fermi levels for electrons $\left(\mu_{e}\right)$ and holes $\left(\mu_{h}\right)$ as obtained from Fermi-Dirac occupation of the densities of states. Here, the charge-carrier density is measured by the fraction $p$ of occupied donor sites. 
suitable device model. Fig. 4.9b also displays photovoltaic gaps $\Gamma$ as the sum of the mean donor IE and acceptor EA: Since the chemical potentials for holes and electrons, $\mu_{e}$ and $\mu_{h}$, lie fairly deep in the respective DOS, the difference $\Gamma-V_{\text {oc }}$ amounts to around $0.6 \mathrm{eV}$ for the mildly disordered systems (P3HT, sexithiophene, pentacene), but grows considerably with energetic disorder, reaching $0.9 \mathrm{eV}$ for $\mathrm{D} 5 \mathrm{M} / \mathrm{C}_{60}$. This finding is supported by a number of studies $[152,157,158]$ where energetic disorder has been hypothesized as a contribution to the observed gap: Experimentally, $\Gamma-V_{\text {oc }}$ has been located at around $0.5 \mathrm{eV}$ [152], but a universality to this relationship has been disproved [158]. This apparent universality is due to the influence of thermal motion on always the same energy scale, $k_{\mathrm{B}} T$. The resulting thermal disorder strengths are typically smaller than $80 \mathrm{meV}$ (see inset in Fig. 4.9b) - up to where the chemical potential at open-circuit conditions lies roughly $0.3 \mathrm{eV}$ below the mean of the DOS, leading to an observed difference to $\Gamma$ of twice that amount when accounting for both donor and acceptor.

A more direct comparison between theory and experiment than in Fig. 4.9b would require the calculation of the illumination-dependent open-circuit voltage, which can only be achieved by explicitly simulating the dynamics of charge generation and recombination [157, 159-162]. Targeting the open-circuit voltage as a function of charge density avoids simulation of recombination rates, mobilities and optical profiles. Due to its simplicity, it provides an equivalent and computationally accessible measure of the energetic capabilities of a donor/acceptor system.

\subsection{Charge Carriers at Grain Boundaries}

So far, we have focused on out-of-plane interfaces where the thin-film and interface normal are by definition aligned. Only in the context of patterned films (Sec. 4.2.1) did we consider systems with in-plane interfaces found to generate in-plane level bending. This case should, however, be termed exotic, since the orientation of the respective domains differed from each other by more than just a uniaxial rotation around the thin-film normal. A grain boundary of this type is not expected in thin devices, as the substrate conditions the orientation of molecules in its vicinity. This preferential orientation results in "uniaxial" grain boundaries, studied in the following due to their role in determining (or rather limiting) the mesoscopic charge-carrier mobility in both polymeric and small-molecular devices [163-165].

Initially, we again limit our description to a lattice level. To generate the morphology, we superimpose up to 16 point patterns, each of which corresponds to a certain crystal alignment on the substrate. The unit cell of the crystals has a $b c c$-structure (lattice constant $c=0.7 \mathrm{~nm}$ ) with a monomolecular basis of $Q_{20}<0$ lattice sites as previously used in Sec. 4.2.1. The individual grains are subsequently grown probabilistically until the simulation cell of $75 \mathrm{x}$ $75 \times 5 \mathrm{~nm}^{3}$ is densely populated based on a site-site exclusion cutoff of $\sqrt{3} / 2 c$. Snapshots of the obtained systems are shown in Fig. 4.11a-d, where the yellow stripes in the top panel indicate the orientation of the domains. The electrostatic contribution $\Delta_{h}^{(1)}$ to the site energy 


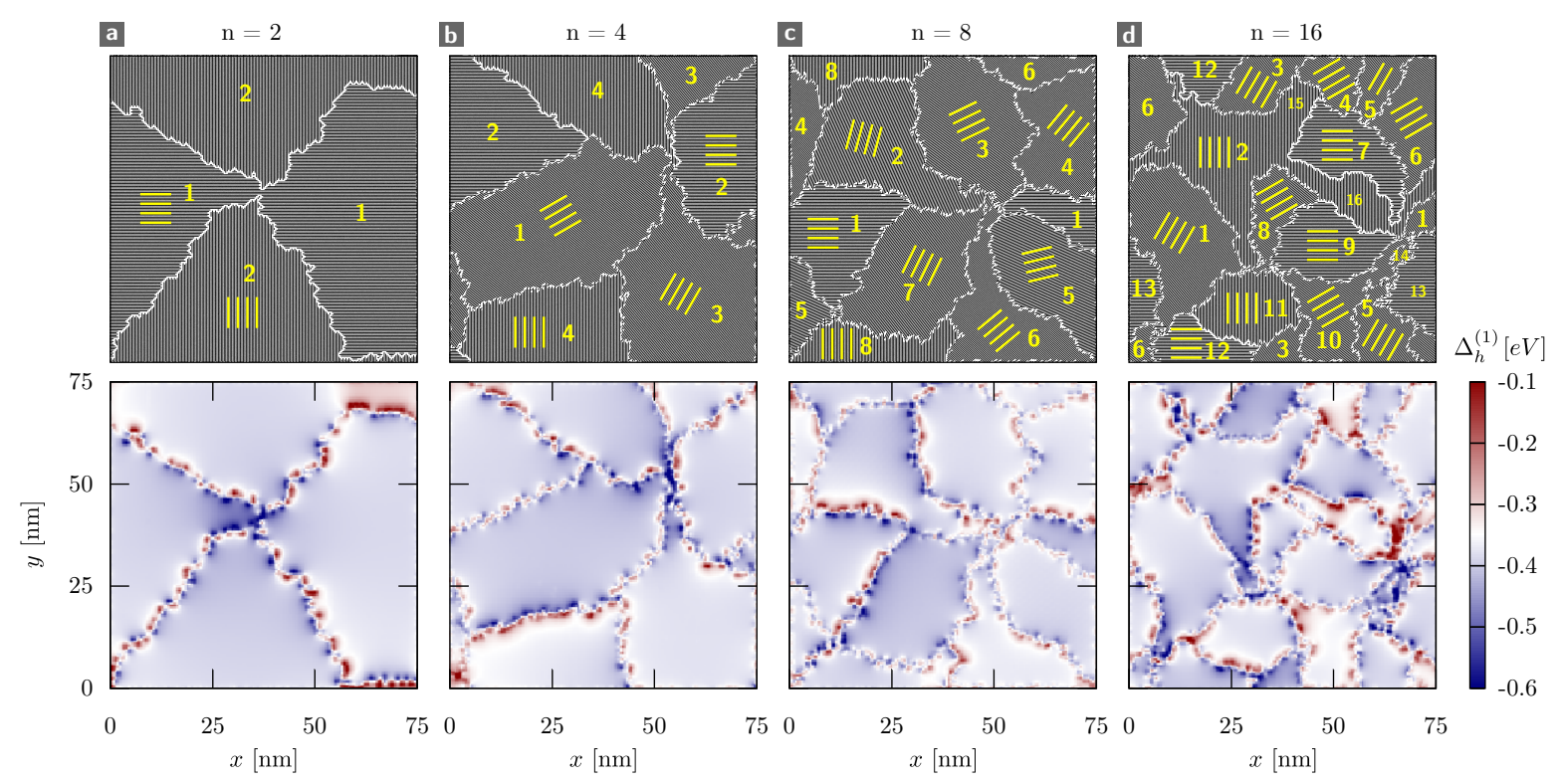

Figure 4.11. $Q_{20} b c c$ lattice grain boundaries. Probabilistically grown lattice grain boundaries with varying seed densities from $n=2$ (a) to $n=16$ (d) seeds. The line blocks in the top panel indicate the orientation of the grains. The bottom panel shows the electrostatic contribution to the energy landscape for holes projected onto the $x y$-plane.

of holes is shown in the bottom panel, projected onto the $x y$-plane. First, it can be seen that the energy level within the domains is approximately constant. This intradomain energy is determined by the out-of-plane component of the quadrupole tensor, which is identical for all grains, as they share the same orientation with respect to the thin-film normal. The energetics at the grain-grain interface is therefore exclusively determined by the short-range interaction of the excess charge with the polar end groups of the neighbouring grain. Due to the crystal packing and $Q_{20}<0$ character of the polar sites, the energy profile across the interface is characterized by the concurrent appearance of a barrier and an adjacent well (in the following referred to as a barrier/well motif), with an only slight dependence of the barrier height and well depth on the grain-grain angle.

It is noteworthy that for electrons, the energy landscape is reversed $\left(\Delta_{e}^{(1)}=-\Delta_{h}^{(1)}\right)$, such that thermal activation is required during the crossing from one grain to the other, independently of the type of the carrier. This observation should be combined with the finding that the energy landscape at the interface is strongly fragmented. Specifically, the energy sequence (barrier/well versus well/barrier) depends on the position along the boundary. In the case of a pure energy barrier at the interface (rather than just a well), the charge carrier is able to cross from one domain to the other without thermal activation, provided it migrates through a gap in the repulsive barrier. An interfacial energy well, by contrast, acts as an attractive trap for charge carriers: The grain-grain interface then impacts the effective activation energy for transport, to be probed by temperature-resolved mobility measurements. 

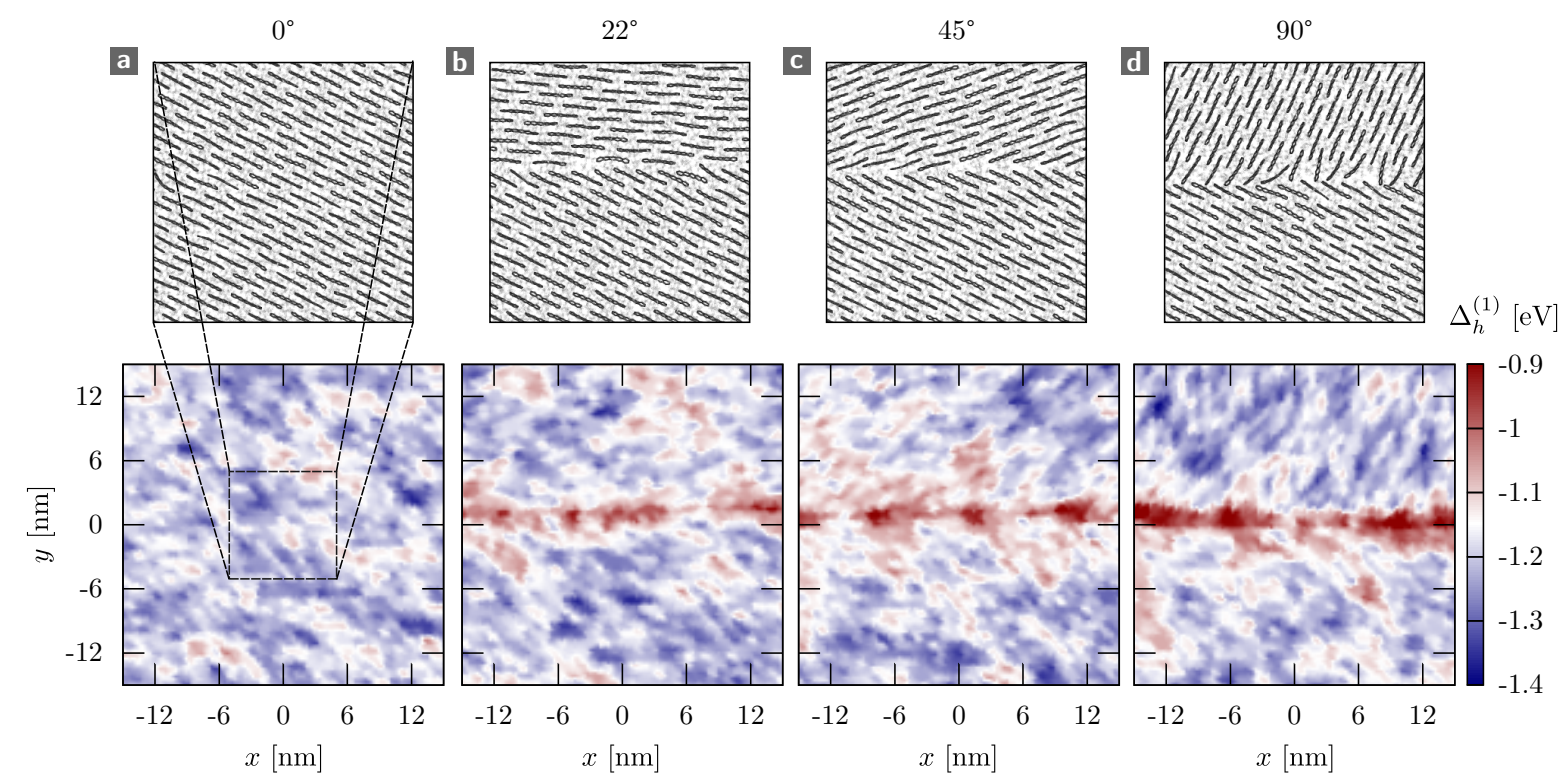

Figure 4.12. Grain boundaries in TIPS-PEN. In-plane grain boundaries with relative orientations of $0^{\circ}(\mathrm{a}), 22^{\circ}(\mathrm{b}), 45^{\circ}$ (c) and $90^{\circ}$ (d). The top panel shows the interfacial packing in the equilibrated systems. Hole energy landscapes in the bottom panel include only the electrostatic contribution $\Delta_{h}^{(1)}$, as the polarization contribution only effects a homogeneous stabilization across the system. The energy barrier is hence due to packing rather than interfacial vacancies that would weaken the dielectric stabilization $\Delta_{h}^{(2)}$.

Depending on molecular architecture and packing, all three interfacial energy motifs (barrier, well, and barrier/well) are conceivable. For the barrier motif, migration across the grain boundary is limited by the time required to access a gap in the barrier, whereas for the well or barrier/well motif, the escape time from the well serves as the controlling time scale for interdomain transport. The barrier motif is hence expected to exhibit the best transport properties - and is indeed realized in the case of hole transport in TIPS-PEN: To illustrate this, Fig. 4.12 displays the $x y$-projected energy landscape of a system composed of two TIPS-PEN grains with four different relative orientations ranging from $0^{\circ}$ to $90^{\circ}$ (see the top panel). Beyond the abrupt barrier increase in between $0^{\circ}$ and $22^{\circ}$, the dependence of the barrier height on orientation is only mild. As explicit simulations of the charge dynamics confirm, however, the grain boundaries from Fig. 4.12a-d can be crossed without a significant impact on mobility, due to barrier gaps that allow for charge crossing with an activation energy that matches the energy for intradomain transport.

We note that the barrier motif observed for holes translates into a well motif for electrons: For the latter, interdomain transport should hence be trap-limited. This relationship points to the importance of low-energy structures and packing motifs, which guarantee efficient charge transport for at least one carrier type by promoting barrier and preventing trap formation at grain-grain interfaces. It remains, however, to be seen to what degree experiments can 
consolidate this picture via temperature-dependent mobility measurements with both holes and electrons in TIPS-PEN and other materials.

\subsection{Summary: Interface Energetics}

In this chapter, we demonstrated how long-range electrostatic effects fostered by mesoscale structural order determine electronic energy levels at organic-organic interfaces. The applied computational framework has been shown to provide accurate results for the energy landscape and open-circuit voltage of a variety of photovoltaic systems.

In particular, we investigated the interplay of molecular architecture, orientation and order, that jointly determine charge-carrier energy profiles. As a direct consequence of longrange molecular ordering, the simulated level profiles are largely flat, as also probed by UPS measurements. In realistic morphologies, this type of molecular organization can result from crystallites forming on the substrate, or simply from preferential alignment of individual molecules induced by the interface in otherwise disordered materials [166]. In fact, anisotropic dielectric properties and, consequently, nematic or smectic ordering are frequently observed in thin organic films [167] and are sufficient to influence the level offset between donor and acceptor domains via uncompensated interface-active quadrupole moments $Q_{20}=Q_{z z}$ and $Q_{22 c} \sim Q_{x x}-Q_{y y}$. Structural coherence in the active layer of bulk heterojunctions will differ from the planar setup, but the nematic (uniaxial) order is retained [62] with the effect that the same energetic mechanisms as for bilayers also apply here. This is supported by the common experimental finding that open-circuit voltages are only slightly larger in the planar layout.

We also investigated the conditions under which in-plane and out-of-plane level bending can be recovered in patterned thin-films or systems with only local structural order. Furthermore, we showed that orientational effects supersede packing effects, investigating how - for the same compound - different out-of-plane quadrupolar moments can cause an electrostatic stabilization or destabilization of charge carriers, irrespective of the packing mode.

The link between molecular order and energy landscape finally rationalizes the success of the DCVnT series and, more generally, of the acceptor-donor-acceptor design, patented by Heliatek [168]. First, only the face-on orientation on $\mathrm{C}_{60}$ provides a level alignment sufficient for charge separation, but not too large to decrease the open-circuit voltage. Second, with the optical transition dipole moment located in the molecular plane, the face-on orientation ensures efficient light absorption. Third, in this orientation, the $\pi$-stacking direction aligns with the interface normal, thus enabling efficient charge transport away from the interface.

This subtle interplay of molecular architecture, orientation, packing and electrostatic effects, that jointly produce a high-performing solar cell, explains why compound design to-date is often only rational ex post - and why in silico screening, using for example methods as presented here, could be of valuable assistance. 


\section{Chapter 5}

\section{Charge Carriers in Disordered Bulk Mesophases}

In this chapter, we extend our study of the density of states (DOS) in organic semiconductors to charge carriers in partially ordered and disordered bulk materials. First, we study DOS alignment and spatial correlations in amorphous phases of dipolar OLED materials, where the long-range treatment applied in this and previous chapters can be exploited to correct for artificial long-range contributions that arise as a finite-size effect in mesoscopically amorphous systems. Second, we address large-scale morphologies and charge transport properties of polymeric mesophases, using a multiscale simulation scheme that incorporates both long-range conformational disorder and local molecular ordering. With poly(3-hexylthiophene) (P3HT) as an example, we illustrate how the energy landscape and its spatial correlations evolve with increasing degree of structural order in mesophases with amorphous, uniaxial, and biaxial nematic ordering. The formation of low-lying energy states in the more ordered systems proves mostly due to larger conjugation lengths rather than electrostatic interactions.

\subsection{Charge Carriers in Amorphous Dipolar Semiconductors}

On the small-molecule level, we have so far investigated the energy landscape of crystalline systems and heterostructures, either in a lattice (Sec. 4.2) or atomistic (Sec. 4.3) description. In electronic devices, these highly ordered systems play a preeminent role in solar cells and transistors, where structural order is required to reduce energetic disorder with its detrimental effect on charge-carrier mobility and device performance. Furthermore, crystalline or partially crystalline systems have an increased tendency to form percolating domains in binary mixtures, thought desirable in solar cells.

In this section, we will investigate amorphous semiconductors used in OLEDs, where disordered materials are preferred due to their processibility and fine miscibility, two criteria that play an important role in host-guest systems. Studying amorphous semiconductors in the context of long-range interactions may appear paradox, since we have already identified 

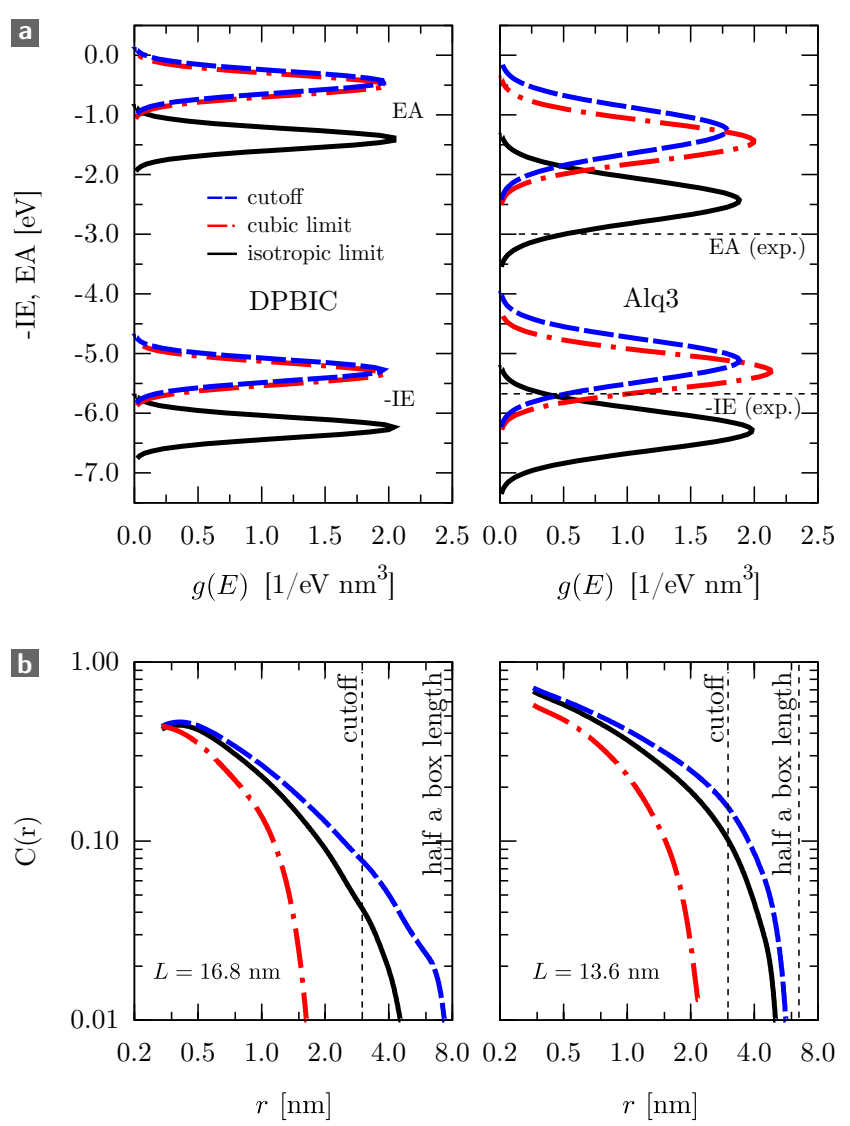

Figure 5.1. Densities of states and spatial correlations in the isotropic limit. (a) Densities of ionization energies and electron affinities in DPBIC (left) and Alq3 (right) computed with a cutoff (red dashed line), and with long-range embedding in the cubic (blue dashed-dotted line) or isotropic limit (black solid line). For Alq3, where experimentally determined energy levels are available, the latter (see solid black lines) yields significantly better agreement with ionization energies and electron affinities extracted from photoelectron spectroscopy $[169,170]$ (dashed lines), where one has to note that photoelectron spectroscopy probes the tail of the densities of states. (b) Spatial correlation function for DPBIC (left) and Alq3 (right) with the computational procedure indicated by the line style as assigned in (a). In the cutoff description, correlations are truncated at the cutoff and in the case of long-range embedding at half the box length, indicative of a finite-size effect. 
(compare also Eqs. 3.41 and 3.42) that uncompensated multipolar moments concatenate the microscopic energetics and mesoscopic order. In amorphous semiconductors, however, these moments should average to zero on large scales to give way to an electrostatically isotropic continuum. Nevertheless, on smaller scales, residual electrostatic ordering may persist and accordingly affect the energy landscape of these materials.

To highlight the computational difficulties that arise from this residual ordering, we investigate two dipolar compounds: the emitter molecule Alq3 [50] and hole conductor DPBIC [28] (see Tab. 1.1 for chemical structures). Atomistic configurations of 4096 molecules were prepared via molecular dynamics simulations with tailored force fields adapted from OPLS-AA. The starting configurations were first randomized at $700 \mathrm{~K}$ temperatures and then quenched to $300 \mathrm{~K}$, with subsequent equilibration over a time period of several nanoseconds. To investigate level positioning, the DOS for holes and electrons (Fig. 5.1a) was simulated using three different computational procedures: a cutoff-based description (dashed blue lines) and the long-range embedding protocol from Chapter 3 with either a cubic shape term (dotteddashed red lines) or no shape term at all (solid black lines). The latter can be interpreted as an isotropic limit, which assumes that all multipolar moments average to zero on a macroscopic scale.

First, we note that the cutoff-based description and long-range description in the cubic limit match closely. This is not surprising in that both frameworks are virtually identical with respect to their long-range behaviour. Notably, a spherical cutoff implicitly includes long-range contributions of the $k=0$ type captured by the shape term from Eq. 3.41, as previously illustrated by the convergence scan from Fig. 3.1b. The comparison between these first two approaches therefore serves as a mere sanity check. If we, however, extend this comparison to the isotropic limit, we observe a striking $1 \mathrm{eV}$ difference in level positioning - proving that this system, though structurally amorphous, features a conditionality in the interaction sum which is picked up by both the cutoff and cubic limit. This observation implies a true challenge for simulations, as it is at this stage impossible to pinpoint the origin of the mesoscopic moments, as they could be due to subtle preferential ordering present also in the real system or to finite-size-induced fluctuations, or both.

If, for example, preferential ordering really plays a role, then how large do system sizes have to be in order to not truncate structural correlation functions before convergence (at least in a thin-film sense) is achieved? Then again, if finite-size effects are exclusively responsible for the observed differences, the isotropic limit should yield the more appropriate description. Indeed, in the case of Alq3, where experimental data is available, this limit appears to agree better with energy levels extracted from the DOS onset $[169,170]$, as indicated by the dashed horizontal lines in Fig. 5.1a.

In addition to level positioning and alignment, we consider the spatial correlation function $C(r)$ of the energy landscape (see Fig. 5.1b), defined as

$$
C(r)=\left\langle\frac{\left(\mathrm{IE}_{i}-\mu_{h}\right)\left(\mathrm{IE}_{j}-\mu_{h}\right)}{\sigma_{h}^{2}}\right\rangle_{r_{i j}=r} .
$$




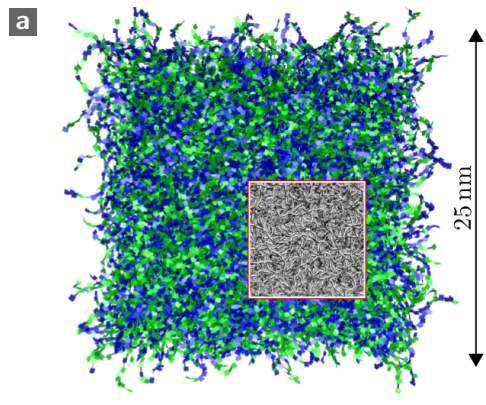

Amorphous

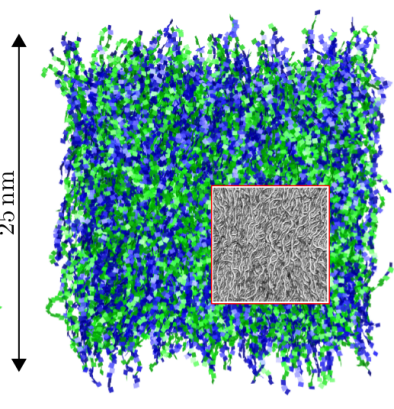

Biaxial-nematic

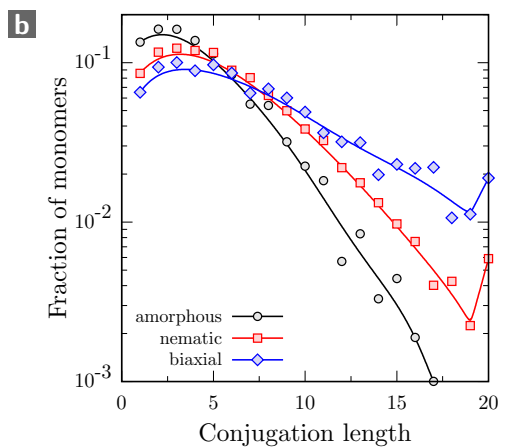

Figure 5.2. Conformational properties of partially ordered mesophases of P3HT. (a) Snapshots of the soft coarse-grained morphologies of amorphous (left) and biaxial-nematic (right) symmetry obtained through simulations by P. Gemünden [72, 74]. The particles of the model (here visualized as plates) represent entire monomers, i.e., a thiophene with a hexyl side chain. The polymer chains are coloured alternatingly in blue and green. (b) Conjugation-length statistics for the amorphous, nematic and biaxial mesophases evaluated after partitioning of polymer chains onto localization units based on a phenomenological torsional criterion for conjugation.

The average $\langle\ldots\rangle$ is performed over all molecular pairs $(i, j)$ with pair distance $r_{i j}$ in the interval $[r, r+\delta r] ; \mu_{h}$ and $\sigma_{h}$ are the average and width of the distribution of ionization energies (IE), respectively. For electron affinities, an analogous expression holds. Correlations of the energy landscape have been shown to result in the characteristic Poole-Frenkel behaviour of the mobility in many organic semiconductors. Fig. 5.1b, however, illustrates that atomistic simulations tend to truncate this correlation function in a cutoff-based description for pair separations larger than the cutoff $r_{c}$ (here: $3 \mathrm{~nm}$ ). Even in a long-range description, the finite system size forces correlations to zero at approximately half the box length, with the cubic limit resulting in stronger correlations.

In amorphous semiconductors, calculating the DOS and spatial correlations thereof may hence be more involved than initially anticipated: In particular, large system sizes (to be tackled with advanced simulation protocols) will be required in order to disentangle finitesize artefacts from ordering effects. In the following section, we will present first steps in this direction in a simulation approach that targets polymeric materials.

\subsection{Charge Carriers in Polymeric Semiconductors}

Functionalized by their conjugated backbones with solubilizing aliphatic side chains, polymeric semiconductors exhibit pronounced self-assembly properties that give rise to complex morphologies characterized by a hierarchical structure: Locally, $\pi$-stacked chains organize into two-dimensional lamellae, which subsequently form three-dimensional crystallites embedded into a partially ordered polymeric matrix. How electronic properties arise from the 
coexistence of these different morphological features is not yet entirely understood [29, 171]. To resolve the factors that limit charge transport in these materials, insight is required into how the kinetics and energetics determine charge-carrier trajectories both within and between disordered and ordered domains.

\subsubsection{Partially Ordered Mesophases of Poly(3-Hexylthiophene)}

Here, we focus on how the density of states evolves with increasing structural order inside partially ordered domains in comparison also with crystalline systems $[60,172]$. The relationship between order and charge-carrier energetics will be exemplified for poly(3-hexylthiophene) (P3HT), a fruit fly of the organic semiconductor community [173]. Historically, charge transport in P3HT has been studied with the aim of relating polymer regioregularity and molecular weight to the morphology, hole mobility, and thus efficiency of bulk heterojunction solar cells. Both hole and electron time-of-flight mobilities were reported to be independent of the molecular weight up to $20 \mathrm{kDa}$, which then decreased by an order of magnitude as molecular weight was further increased to $120 \mathrm{kDa}$ [174]. The field-effect mobility was found to increase with the molecular weight in spite of reduced crystallinity. This was attributed to either better interconnectivity of the polymer network [175] or smaller intrachain ring torsions present in high molecular weight molecules [176].

Due to the slow dynamics of polymeric systems, computational approaches that address mesoscopic chain ordering have to rely on drastic coarse-graining procedures. The morphologies addressed in the following (see Fig. 5.2a) are based on the soft coarse-grained model developed by P. Gemünden and K. Daoulas [72] (see Sec. 2.2.4 for details): Starting from a particle representation of entire monomers that interact via soft anisotropic potentials, an atomistic description is obtained through sequential backmapping of the coarse-grained structures. In the simulation of charge transport - a process affected by morphological features across multiple scales - the resulting, large-scale, atomistic morphologies prove essential: They incorporate both the atomistic structure of the material (entering site energetics and electronic couplings) and the mesoscopic ( $\gtrsim 10 \mathrm{~nm}$ ) polymer alignment (enabling fast chargecarrier motion along the conjugated backbone [171, 175]). To simulate charge transport in these partially ordered morphologies, there is in principle no alternative to computing the electronic wave function of the entire system. Since the excited state of the charged system cannot be computed even with modern quantum-chemical methods, all approaches to evaluate the wave function of large systems have focused on neutral polymers and thus interpret one-electron states as charge localization sites [173]. As a result, electronic and nuclear polarization effects due to the excess charge have not been taken into account. The primary focus of this section will hence be to assess and quantify the corresponding intermolecular, perturbative contributions to the DOS.

In order to additionally describe the effect of chain ordering on charge-carrier properties, we have analysed three types of molecular ordering: amorphous, uniaxial nematic, and biaxial nematic. We obtain the DOS of these systems by first partitioning the polymer backbone onto 

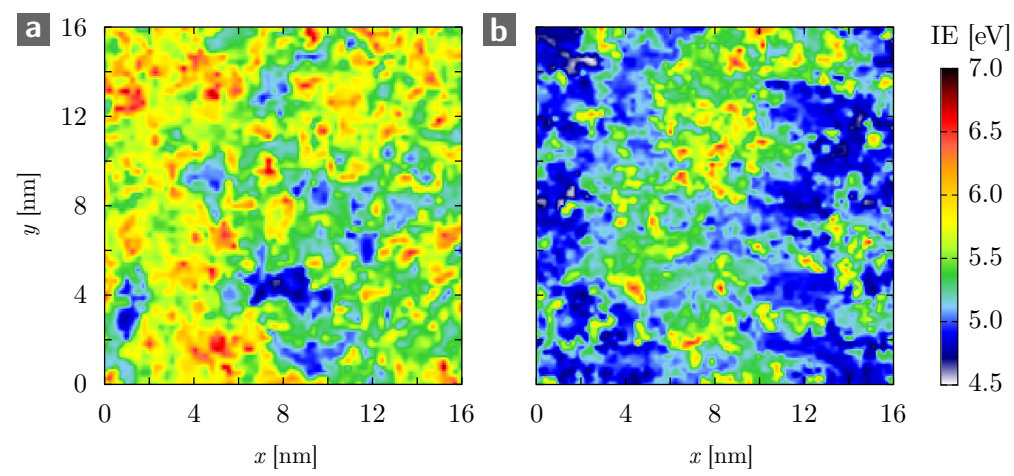

Figure 5.3. Energy landscape for hole transport. Projection of the ionization-energy landscape over a $2 \mathrm{~nm}$-thick slice through the amorphous (a) and biaxial (b) mesophase. Formation of extended low-IE next to defect-laden regions sets energetics in the biaxial system apart from the amorphous case. In the biaxial case, the nematic director coincides with the $x$-axis.

localization units, and subsequently evaluating the ionization energy for each unit, taking into account electrostatic and inductive contributions that result from the interaction of the hole with the molecular environment.

The partitioning onto localization units was performed by imposing a phenomenological criterion for conjugation (see Sec. 2.3): If the torsional deviation from planarity between bonded thiophene monomers exceeds $\pm 45^{\circ}$, conjugation between those two monomers is assumed broken [72]. An empirical assessment of this kind has been indicated to fail in some cases $[86,173]$, but still provides a reasonable approximation to the distribution of conjugation lengths through identification of sufficiently planar segments. Fig. 5.2b reflects this distribution via the fraction of monomers that form part of a conjugated segment of length $l$. As expected, with increasing ordering from amorphous via nematic to biaxial, the frequency of small conjugation lengths $(l \leq 6)$ decreases in favour of larger conjugation lengths $(l>6)$. Additionally, the nematic and biaxial mesophases both exhibit a small jump in frequency towards fully conjugated chains, which in the biaxial case is, however, significantly reduced compared to the initial soft coarse-grained model, in line with the partial loss in biaxial order upon backmapping.

\subsubsection{Formation of Low-Energy States and Correlations}

With the systems partitioned onto localization units, we turn to the resulting energy landscapes. Here we have again employed the long-range corrected perturbative treatment as developed in Chapter 3, where molecular electrostatic potentials and the molecular polarizability are parametrized via atom-centred distributed multipoles and polarizabilities, respectively [50]. An account of long-range interactions is desired to capture all effects that accompany non-local ordering. Here it is realized by embedding a $3 \mathrm{~nm}$ polarization cloud around the charged segment in a periodic prepolarized background that defines the polar- 

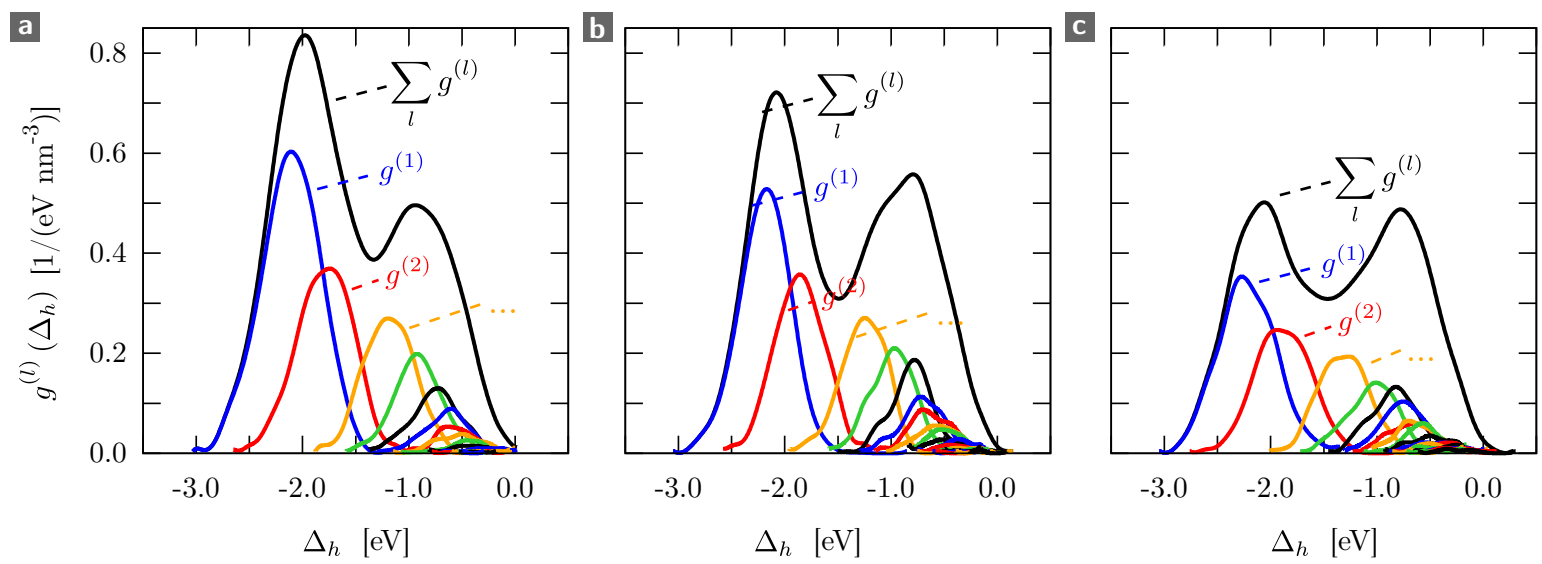

Figure 5.4. Conjugation-length decomposition of the density of states. Intermolecular density of states $g^{(l)}$ computed from the electrostatic and polarization contribution $\Delta_{h}$ to ionization energies without the intramolecular (gas-phase) contribution, resolved according to segment length $l$, for the (a) amorphous, (b) nematic and (c) biaxial phase.

ization state of the ground-state system. Field interactions are treated in an Ewald-type fashion, while taking into account self-consistent polarization. Additionally, a cube shapeterm as previously used in Sec. 5.1 removes the conditional convergence that can result from the interaction of a charge with a net-quadrupolar environment. Note that this procedure neglects nuclear polarization of the environment. The internal reorganization energy, ranging between $0.2 \mathrm{eV}(l=1)$ to $0.05 \mathrm{eV}(l=20)$ for a planar molecular conformation, should, however, serve as a generous upper limit for this additional stabilization.

Altogether, the ionization energy (IE) is the sum of three terms, the gas-phase ionization energy evaluated for a P3HT chain of length $l$ via density functional theory (B3LYP/6-311g) plus the first-order electrostatic and second-order polarization contributions. As before, we will denote the sum of the latter two as $\Delta_{h}$. The associated intermolecular DOS $g^{(l)}\left(\Delta_{h}\right)$ (this excludes the intramolecular contribution to the site energy) is resolved according to conjugation length in Fig. 5.4a-c. It can be seen that the polarization component is greatly impacted by the higher excess charge density in shorter conjugation units, leading to a larger stabilization. Taking into account the internal contribution, we will see that this concentration effect results in a considerable narrowing of the total DOS.

Before doing so, we will first qualitatively inspect the ionization energy landscape shown in Fig. 5.3, projected over a $2 \mathrm{~nm}$ slice of the amorphous and biaxial systems: The amorphous mesophase is characterized by a strongly fragmented energy landscape, whereas the biaxial system, with chains preferentially oriented along the $x$-axis, displays extended low-IE regions that align with the nematic director, next to high-IE islands where defects tend to cluster. These defects are, however, primarily the result of conjugation defects rather than strong fluctuations in molecular fields.

To arrive at a quantitative picture, we disentangle the spatial and distributional compo- 
nents by individually considering the total DOS (Fig. 5.5a) and its spatial correlation function (Fig. 5.5b). In the former, we observe a decrease of the monomer peak at high IEs (Fig. 5.5a) and simultaneous extension of the DOS towards lower IEs, with increasing structural order from amorphous via nematic to biaxial. In fact, due to the stronger delocalization of charges in the low-IE region and hence larger volume associated with those states, the change from amorphous to biaxial is more drastic than it may appear from considering the DOS alone. We note that the evolving shoulder at $5 \mathrm{eV}$ (see also the inset) plays a crucial role for charge transport and highlights why in semicrystalline polymers amorphous regions will not easily participate in charge transport [171].

It is furthermore instructive to consider the conjugation-length-resolved energetic disorder $\sigma_{l}$ and energetic mean $\mu_{l}=\langle\mathrm{IE}\rangle_{l}$, shown in Fig. 5.5c-d, respectively. Indeed, both $\sigma_{l}$ and $\mu_{l}$ plateau for conjugation lengths $l \gtrsim 6$. For $\sigma_{l}$, this behaviour is understood based on the correlation length of local (e.g., dipolar) fluctuations of the electric potential: Longer segments experience the averaged effect of these local perturbations, whereas short segments are subjected to the full effect of local disorder. For $\mu_{l}$, the plateau results from the opposed action of increased delocalization (which reduces the internal IE) on the one hand and reduced inductive stabilization on the other hand. These length-compensating effects prove that the energy landscape is less sensitive to conjugation length than anticipated, provided an average conjugation length $l \gtrsim 6$. Interestingly, the mean of the DOS in crystalline P3HT in a faceon thin-film setup, studied via the same approach, is located at $4.75 \mathrm{eV}$ and hence overlaps significantly with the DOS from Fig. 5.5a. However, energetic disorder that accompanies paracrystallinity is significantly reduced in these crystalline regions, measuring only $0.06 \mathrm{eV}$, compared to $0.2 \mathrm{eV}$ observed in the partially ordered phases (Fig. 5.5b). It is hence expected that energetic disorder plays an even larger role than the energetic mean in setting apart the charge transport properties of ordered and partially ordered domains.

Finally, we turn to the spatial correlation function, which we define as an average over the correlation function of individual subpopulations of length $l$ :

$$
C(r)=\left\langle\sum_{l} \frac{\left(\mathrm{IE}_{i(l)}-\mu_{l}\right)\left(\mathrm{IE}_{j(l)}-\mu_{l}\right)}{\sigma_{l}^{2}}\right\rangle_{r_{i j}=r}
$$

$C(r)$ is plotted for all three mesophases in Fig. 5.3b. Indeed, the correlation function decays slower the more ordered the system, an effect that can be traced back to the increased long-range order that was inherited from the soft model. In biaxial systems, a weak spatial correlation of site energies already resulted from the spatial partitioning into ordered and less ordered regions [72]. Long-ranged electrostatics, however, significantly amplifies the effect of structural order on site-energy correlations.

Despite increased conjugation in the biaxial-nematic phase, the DOS still exhibits clear qualitative differences from its crystalline counterpart. In crystalline domains, the narrowing of the DOS results primarily from the formation of $\pi$-stacked lamellae. The absence of such structures in the polymeric mesophases studied here becomes most prominent upon inspection 

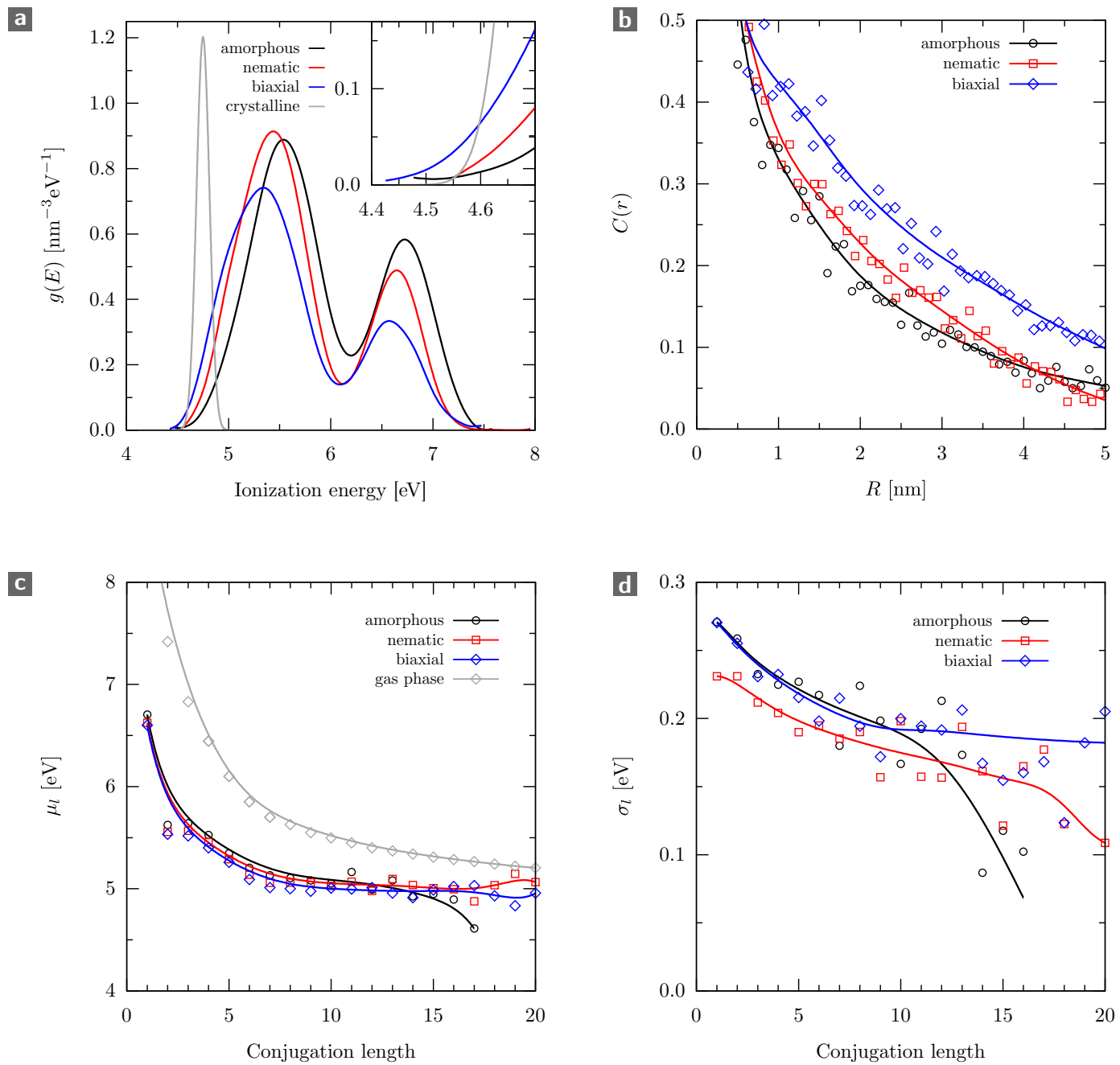

Figure 5.5. Formation of the low-energy tail, energetic disorder and spatial correlations. (a) Hole density of states for the amorphous, nematic and biaxial mesophase. With increasing structural order, the monomer peak decreases in favour of a developing low-IE shoulder around $5 \mathrm{eV}$. (b) Spatial site-energy correlation function $C(r)$ for the amorphous, nematic and biaxial mesophases. The increase of spatial correlations is the combined effect of long-range order - most pronounced for the biaxial system - and electrostatic interactions. (c) Conjugation-length resolved energetic disorder $\sigma_{l}$ and (d) energetic mean $\mu_{l}$. Both $\sigma_{l}$ and $\mu_{l}$ plateau as of conjugation lengths $l \gtrsim 6$. Note that the drop in $\sigma_{l}$ observed in the amorphous and nematic system for large $l$ is due purely to poor statistics. 


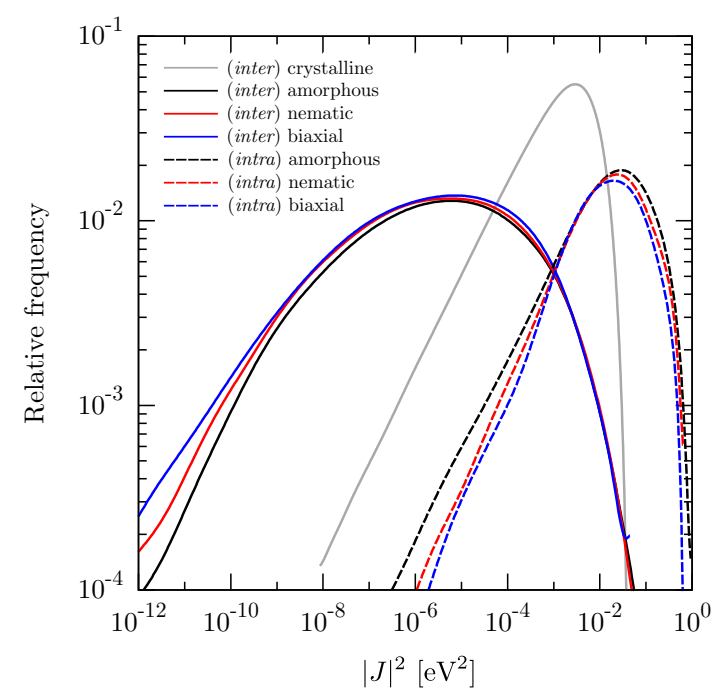

Figure 5.6. Electronic couplings for holes in different phases of P3HT. Intermolecular (solid lines) and intramolecular (dashed lines) electronic coupling elements $|J|^{2}$ for the amorphous (black), nematic (red) and biaxial (blue) mesophases. Also shown are the intermolecular couplings elements computed for a P3HT crystal (polymorph I), with the significant boost in coupling strength resulting from the organization into $\pi$-stacked lamellae.

of the distribution of electronic couplings (Fig. 5.6): Beyond a slight increase of the relative frequency of intermolecular (solid lines) as opposed to intramolecular (dashed lines) couplings from the amorphous to biaxial case, there is no significant difference between the three systems. Comparing the situation to the crystalline case (grey curve), one nevertheless notices that locally, high intermolecular couplings are attainable to the degree that a percolating network is formed.

\subsection{Summary: Mesophase Energetics}

In this chapter, we investigated the effect of partial ordering on the bulk charge transport properties of organic semiconductors.

To systematically identify ordering effects in polymeric materials, we studied chargecarrier energetics across a sequence of mesophases with gradually increasing degree of molecular order - from completely amorphous via nematic uniaxial to biaxial liquid-crystalline. A three-step hierarchical scheme capable of generating large-scale morphologies enabled atomistic access to electronic properties: Initially, morphologies are equilibrated on large scales using a soft coarse-grained model with anisotropic non-bonded interactions between particles representing entire repeat units. Subsequently the resolution is refined via an intermediate model derived from systematic coarse-graining that facilitates the insertion of all-atom details in the final step. Applying the procedure to P3HT as a test system, we computed the energy landscape for holes in the resulting morphologies. With increasing structural order, low- 
energy states and spatial correlations amplify, with conjugation-length independent energetic disorder and energetic mean as of a moderate segment length of six repeat units. In comparison to crystalline systems, these findings indicate that the energy landscape of crystalline systems promotes efficient transport primarily because of the reduced energetic disorder that accompanies lamellar packing.

Our study of mesophases of small-molecular, dipolar materials revealed that long-range effects even persist in structurally amorphous systems, as either a finite-size induced simulation artefact or a result of residual, structural correlations, or both. The long-range treatment can be exploited to partially correct for finite-size effects. Nevertheless, the disentangling of artificial and real fluctuations of residual electrostatic moments presents a considerable challenge for the description of stacked devices composed of thick, partially ordered films - notably transport and host:guest emission layers in OLEDs, where tuning of level alignment is crucial in order to guarantee device functionality and bypass degradation mechanisms [100]. 


\section{Chapter 6}

\section{Charge Transfer States at Donor- AcCeptor Heterojunctions}

Organic solar cells rely on the conversion of a Frenkel exciton into free charges via a charge transfer state formed on a molecular donor-acceptor pair. These charge transfer states are strongly bound by Coulomb interactions, and yet efficiently converted into charge-separated states. In this chapter, we show how long-range molecular order and interfacial mixing generate homogeneous electrostatic forces that can drive charge separation and prevent minoritycarrier trapping across a donor-acceptor interphase. Comparing a variety of small-molecule donor-fullerene combinations, we illustrate how tuning of molecular orientation and interfacial mixing leads to a tradeoff between photovoltaic gap and charge-splitting and detrapping forces, with consequences for the design of efficient photovoltaic devices. Drawing from both simulation and experimental results, we also investigate the empirical relationship between the temperature- and charge-density-dependent open-circuit voltage and charge transfer state energy.

\subsection{Pathway for Charge Splitting and Detrapping}

The efficiency of organic solar cells can be expressed as the product of the open-circuit voltage $\left(V_{\mathrm{oc}}\right)$, short-circuit current $\left(J_{\mathrm{sc}}\right)$ and fill factor $(\mathrm{FF})$. These quantities have complex interdependencies, as they derive from just a few elementary processes. Mechanisms that enhance $J_{\mathrm{sc}}$, for example, connect with strong absorption, high charge-carrier mobilities, and efficient charge extraction. Building on the success of the bulk heterojunction concept, strategies in pursuit of higher short-circuit currents therefore employ light-absorbing non-fullerene acceptors [177, 178], low-band-gap polymers [30], materials for singlet-exciton fission [179, 180], or triple-layer energy-relay cascade structures [39] as building blocks.

Recipes that target $V_{\text {oc }}$ involve either morphological tuning [181, 182], insertion of interlayers [183] or manipulations of the chemical structures of the active materials [184]. Furthermore, in an attempt to prescreen suitable donor-acceptor combinations, a correlation of $V_{\mathrm{oc}}$ 
with the sum of the gas-phase ionization energy $\left(\mathrm{IE}_{0}\right)$ of the donor and electron affinity $\left(\mathrm{EA}_{0}\right)$ of the acceptor is typically assumed. However, such a correlation is coincidental rather than systematic, as it neglects the impact of molecular fields that in turn depend on the molecular packing and orientation in the solid state.

A rigorous evaluation of the thermally broadened density of states of donor and acceptor species as pursued in Chapter 4, by contrast, shows that calculation of the charge-densitydependent (as opposed to illumination-intensity-dependent) $V_{\text {oc }}$ is possible [106]. Regrettably, such an approach can still be misleading, as it imposes a finite steady-state charge density and hence does not verify whether charges are generated in the first place. For illustration, consider the level schematics presented in Fig. 6.1a-b: Both pertain to the interface between $\mathrm{C}_{60}$ and the merocyanine dye EL86, in its tip-on (Fig. 6.1a) and face-on (Fig. 6.1b) orientations. With sizeable level offsets, comparable $E_{\text {ct }}$ and a gas-phase Frenkel exciton energy of $2.8 \mathrm{eV}$, both configurations (a) and (b) appear suitable for a solar cell. Using correlations established between photovoltaic gap $\Gamma$, CT-state energy and $V_{\mathrm{oc}}$, the face-on configuration is hence expected to yield an open-circuit voltage that is roughly $0.6 \mathrm{eV}$ larger than in the tip-on scenario. And yet, we will rationalize in this chapter why the face-on scenario does not yield an efficient (i.e., charge-generating) interface at all.

More generally, we will address three questions. First, what is the maximum $V_{\text {oc }}$ that can be harvested from a given donor-acceptor pair? Second, which interfacial morphology is required to retain a functional device? Third, how can charge transfer (CT) states split up fast and efficiently? All three questions are ultimately linked to the (still controversial) energetics of charge-transfer and charge-separated (CS) states at donor-acceptor heterojunctions: As an example, the CT-binding energy $\left(E_{\mathrm{ct}}\right)$ calculated for ideal interfaces is of the order of $0.3 \mathrm{eV}[99,185]$, even in high-performing systems such as the $8.3 \%$-efficient oligothiophene derivative DCV5T-Me(3,3) (D5M) combined with $\mathrm{C}_{60}$ (studied in Sec. 4.1).

With $E_{\text {ct }}$ approximately ten times larger than the room-temperature $k_{\mathrm{B}} T$, fast and efficient charge-separation appears unlikely. Charge delocalization, though helpful, would draw fewer benefits from dielectric solvation and not necessarily neutralize the Coulomb attraction in such a way that charges separate without the need for thermal activation $[186,187]$. In particular, the weak intermolecular couplings in both polymeric and small-molecular systems composed of strong dyes are too weak to promote delocalization. Similarly, neither entropydriven separation [136] nor long-range charge transfer [138] boost rates to an extent that justifies an ultrafast process. Continuous level bending has been proposed as a further pathway for cold-exciton break-up [97], but it would still lead to locally bound CT states, and, more importantly, will not necessarily hold in the presence of mesoscale order, which yields flat level profiles [106].

Pathways for charge separation as a microscopically poorly understood process $[134,136$, 138, 161, 186-189] are therefore still actively investigated: As a key result of this chapter, we will show how the energy landscape that emerges from mesoscale order provides pushout forces that can drive the charge separation process - in line with the apparent absence of a Coulomb barrier claimed for some systems [187]. In deriving the functional difference 

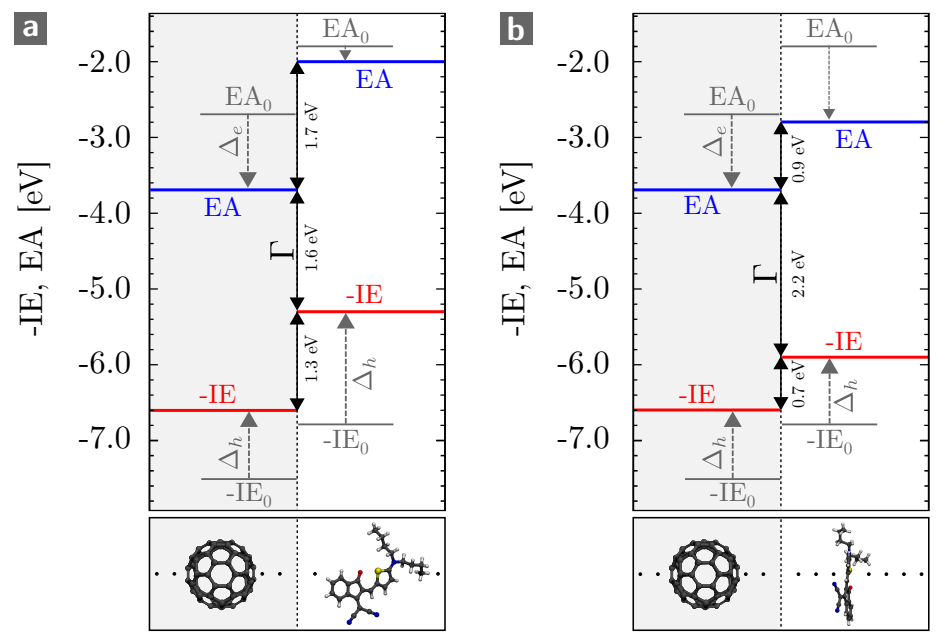

Figure 6.1. Level schematics for $\mathbf{C}_{60} /$ EL86. (a) Tip-on orientation of EL86 on $\mathrm{C}_{60}$ with a small photovoltaic gap $\Gamma$ versus the (b) face-on orientation with large $\Gamma$. Gas-phase ionization energies and electron affinities are denoted as $\mathrm{IE}_{0}$ and $\mathrm{EA}_{0}$, respectively. $\Delta_{e}\left(\Delta_{h}\right)$ are the intermolecular electrostatic and polarization contributions to electron (hole) energies, computed for interfaces composed of $10 \mathrm{~nm} \mathrm{C}_{60} / 10 \mathrm{~nm}$ EL86, with the orientation of EL86 as sketched in the bottom panels.

between chemically versus electrostatically generated level offsets, we rationalize why donoracceptor intermixing can be beneficial for a functioning device, and identify tradeoffs and structure-energy relationships helpful in the design of solar-cell materials.

\subsubsection{Dielectric Solvation of Electron-Hole Pairs}

Before looking into pathways for charge separation, we will first rationalize why the $0.3 \mathrm{eV}$ claimed in the introduction for the $\mathrm{CT}$ binding energy at ideal $\mathrm{C}_{60} / \mathrm{D} 5 \mathrm{M}$ interfaces in fact serve as a generic energy scale for $E_{\text {ct }}$ across many compounds. To this end, we again employ the embedding procedure detailed in Chapter 3: As already practiced in Chapters 4 and 5, the solid-state contributions $\Delta_{s}$ to the site energies of molecular excitations ( $s=h, e$, ct for holes, electrons and CT states, respectively) follow from the perturbative corrections $W$ via $\Delta_{s}=W_{s}-W_{n}$, relative to the neutral ground state $(n)$.

We saw previously that the interaction sum associated with a net charge embedded in a net-quadrupolar environment is in general only conditionally convergent $[106,122])$. In a thin-film setup, the $1 / r^{3}$ character of the charge-quadrupole interaction still results in a rather slow convergence, with the effect that the energetics is tied to the degree of mesoscale order. Even though CT states are net-neutral excitations, we will again explicitly account for all long-range electrostatic interactions to which the excitations are subjected. This is made necessary by the finite spacing of around $1 \mathrm{~nm}$ between the CT hole and electron. CT states should hence be regarded as more than just a dipolar excitation, in particular because they are formed across a donor-acceptor interface. As we have observed in Chapter 4, the quadrupolar 

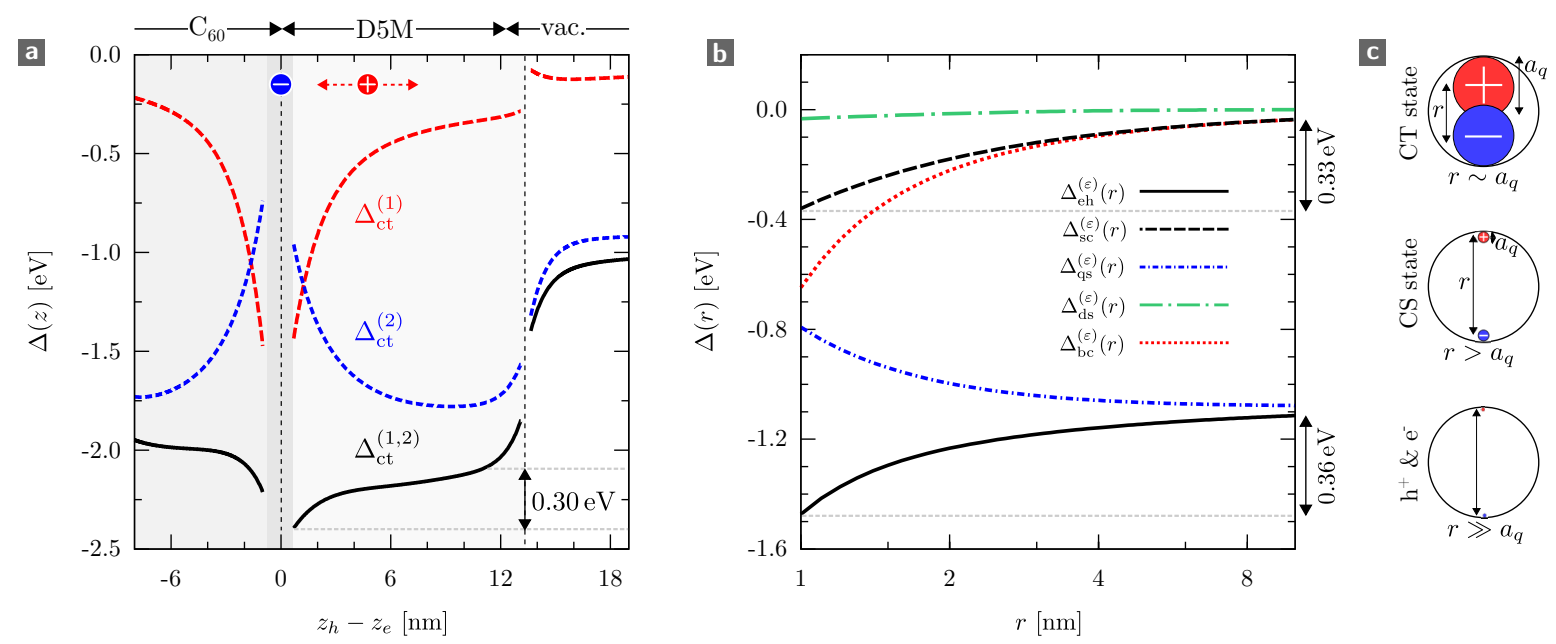

Figure 6.2. Two-carrier hole-electron energy landscape. (a) Cross-section through the hole-electron correlation map in $\mathrm{C}_{60} / \mathrm{D} 5 \mathrm{M}$ for the electrostatic and polarization correction $\Delta_{e h}$ to the energy landscape, resolved according to the electrostatic $\left(\Delta_{e h}^{(1)}\right)$ and inductive $\left(\Delta_{e h}^{(2)}\right)$ contributions. The larger stabilization of the CS compared to CT state reduces the net CT binding energy to around $0.3 \mathrm{eV}$. (b) Electron-hole energies $\Delta_{e h}^{(\varepsilon)}(r)$ in the continuous dielectric solvation model, including the bare coulomb attraction $\left(\Delta_{b c}^{(\varepsilon)}\right)$, dipole stabilization $\left(\Delta_{d s}^{(\varepsilon)}\right)$ and charge stabilization $\left(\Delta_{q s}^{(\varepsilon)}\right)$. In spite of its distinct physical origin, the resulting net electron-hole attraction can be effectively modelled by a screened interaction potential $\Delta_{s c}^{(\varepsilon)}$. (c) Transition from charge transfer to charge-separated state in the dielectric solvation model with a radius-dependent dielectric constant of the charged cavity. For the calculation in (a), $a_{q}=1 \mathrm{~nm}$ and $\varepsilon_{0}=4$. 
background generates an abrupt step in the electrostatic potential across this interface, as opposed to continuous electrostatic profiles that result from a more local description. This electrostatically generated offset can either amplify or reduce the offset between electron and hole levels on the donor and acceptor side. In general, the two poles of the CT state are hence subjected to a different electrostatic environment. A long-range treatment that accounts for the preferential alignment of molecules on a mesoscale is therefore indispensable.

For the $\mathrm{C}_{60} / \mathrm{D} 5 \mathrm{M}$ interface studied in Sec. 4.1, we will now investigate the energy landscape of a single electron-hole pair. For our current purpose, it is sufficient to study a onedimensional slice through this six-dimensional landscape by varying only the $z$-coordinate of the CT hole, while constraining the CT electron to the interfacial acceptor unit. The effective potential seen by the hole is shown in Fig. 6.2a. The electron at $z_{e}=-0.5 \mathrm{~nm}$ leads to an attractive well in the total potential (black curve). This attractive well is, however, neither given by the bare nor screened Coulomb potential as becomes clear upon inspection of the electrostatic (blue curve) and polarization (red curve) contributions: Together, they result in an effective barrier for charge separation of approximately $0.3 \mathrm{eV}$. Individually, however, they experience an almost $1 \mathrm{eV}$ change during the transition from a CT to CS state, characterized by an increasing $\Delta_{\mathrm{ct}}^{(1)}$ and decreasing $\Delta_{\mathrm{ct}}^{(2)}$.

The behaviour of $\Delta_{\mathrm{ct}}^{(2)}$ already indicates that the "conventional" intuition behind the $1 / \varepsilon$ charge-charge screening is not suited to account for the reduction of the CT binding energy from around $1.4 \mathrm{eV}$ (corresponding to the binding energy of two point charges at a separation of $1 \mathrm{~nm}$ ) to $0.3 \mathrm{eV}$. To highlight this, we resort to a simple dielectric cavity model: In this model, we describe the lack of dielectric material to screen the charges at small separations by a distance-dependent dielectric constant $\varepsilon_{c}$ of the cavity that encompasses the two charges with separation $\left|z_{h}-z_{e}\right|=r$ :

$$
\varepsilon_{c}(r)=\varepsilon-\frac{2 a_{q}^{3}(\varepsilon-1)}{\left(\frac{1}{2} r+a_{q}\right)^{3}}, r \geq 2 a_{q} .
$$

The latter expression follows from a volume ratio argument, where $a_{q}$ (see Fig. 6.2c) is the size of the molecular units, $\varepsilon$ is the dielectric constant of the surrounding material. Within this model, the total contribution to the electron-hole-pair energy reads

$$
\Delta_{e h}^{(\varepsilon)}(r)=\Delta_{b c}^{(\varepsilon)}(r)+\Delta_{q s}^{(\varepsilon)}(r)+\Delta_{d s}^{(\varepsilon)}(r)
$$

$\Delta_{b c}^{(\varepsilon)}(r)$ incorporates the bare Coulomb interaction screened only by the distance-dependent $\varepsilon_{c}(r) . \Delta_{q s}^{(\varepsilon)}(r)$ and $\Delta_{d s}^{(\varepsilon)}(r)$ are the stabilization of the CT charge and dipole component, respectively [125]:

$$
\Delta_{q s}^{(\varepsilon)}(r)=2\left[-\frac{1}{8 \pi \varepsilon_{0}} \frac{\varepsilon_{c}(r)-1}{\varepsilon_{c}(r)} \frac{q^{2}}{a_{q}}\right],
$$




$$
\Delta_{d s}^{(\varepsilon)}(r)=-\frac{1}{8 \pi \varepsilon_{0}} \frac{2\left[\varepsilon-\varepsilon_{c}(r)\right]}{\varepsilon_{c}(r)\left[\varepsilon_{c}(r)+2 \varepsilon\right]} \frac{r^{2} q^{2}}{\left(\frac{1}{2} r+a_{q}\right)^{3}} .
$$

The resulting effective energy landscape exhibits the same behaviour as observed for the atomistic calculation (see Fig. 6.2b), even though the absolute positioning of the curves differs due to the absence of explicit molecular fields in the dielectric model. For $a_{q}=1 \mathrm{~nm}$ and $\varepsilon=4$, the CT binding energy approaches $0.4 \mathrm{eV}$ (see black solid line). The lowering of the Coulomb barrier again results from the exchange of Coulomb attraction for dielectric stabilization during the conversion from a CT to a CS state. The electron-hole pair hence undergoes what has been called dielectric solvation [185]. We note that the reaction-field potential $\Delta_{s c}^{(\varepsilon)} \sim 1 /(\varepsilon r)$ (dashed black line in Fig. 6.2) produces similar binding energies and can thus serve as an effective description for the charge separation process. When parametrized on an atomistic reference as shown in Fig. 6.2a, the latter could prove useful for multi-carrier kinetic Monte Carlo simulations, where a full-blown, self-consistent evaluation of the electron-hole interaction is too expensive to be performed on the fly.

We have now justified why CT binding energies are typically located on a scale of 0.3$0.4 \mathrm{eV}$, which is still difficult to access thermally. Mechanisms that lower this binding energy are therefore desirable. One particular pathway that harnesses interfacial fields in structurally ordered systems for charge splitting will be proposed in the following section.

\subsubsection{The Role of Interfacial Defects}

To understand how electrostatics can promote barrier-less CT separation through the action of long-range electrostatic fields, we consider a donor-acceptor interphase, that is, an interfacial region characterized by intermixing of the donor and acceptor molecular species. Absent at sharp heterojunctions, this type of interphase has been found to improve exciton yield in polymer-fullerene devices $[190,191]$ - i.e., disorder appeared to be of advantage. We expand on this observation and study, as a proof of concept, small protrusions of $\mathrm{C}_{60}$ into a D5M domain. The atomistic model, equilibrated via molecular dynamics, consists of a $\mathrm{C}_{60}$ substrate and D5M film, each of $10 \mathrm{~nm}$ thickness. The total interfacial area amounts to $40 \mathrm{~nm}^{2}$. The protrusion takes up approximately $10 \%$ of this area.

Simulation results for the atom-resolved environment contribution $\Delta_{e}$ to electron affinities are shown in Fig. 6.3a. In the projection of the energy landscape of the fullerene region, averaging is performed over a $2 \mathrm{~nm}$ slice that fully incorporates the $\mathrm{C}_{60}$ appendix. Notably, the abrupt step in $\Delta_{e}$ across the interface, which results from the coherent action of quadrupolar fields of the D5M, persists, as these fields are generated non-locally, i.e., they are the combined effect of millions of preferentially oriented molecules that together define the electrostatic surrounding on a mesoscale. Note that, as discussed for charge carriers, a uniaxial thin-film symmetry (implying nematic order) with a coherence length far larger than the film thickness is already sufficient to foster long-range quadrupolar fields. This ordering is typically realized for polycrystalline materials, where each crystallite adopts a preferential out-of-plane (edge-on, tip-on, ...) orientation on the substrate, whereas the in-plane orientations differ 
a
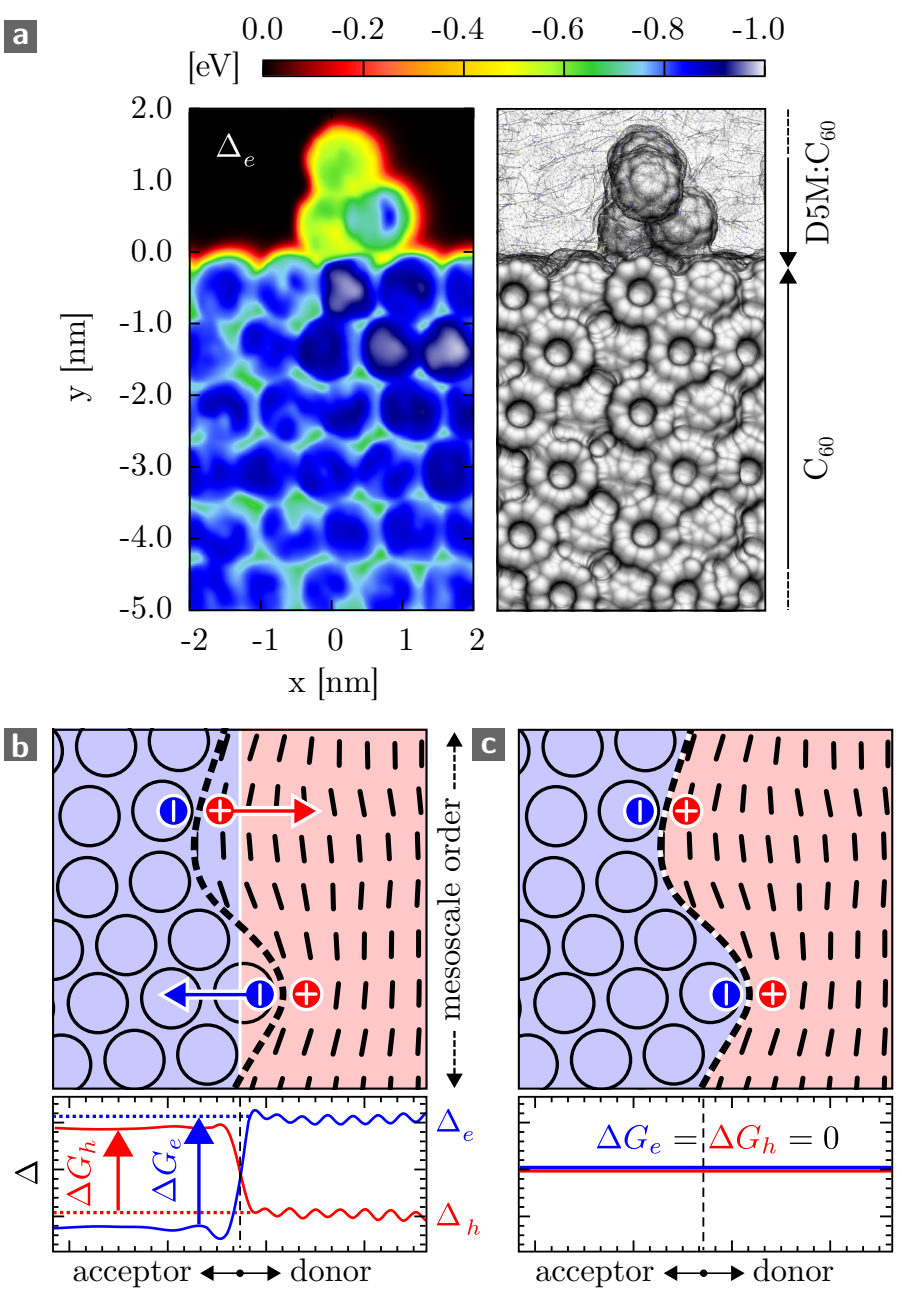

Figure 6.3. Charge push-out in $\mathrm{C}_{60}$ /D5M. (a) Colour map of the projected electrostatic and polarization contribution $\Delta_{e}$ to electron affinities. The atomistic morphology accommodates a small fullerene protrusion into the D5M donor domain. This protrusion is subjected to mesoscale fields generated within the donor majority domain. The resulting driving force for charge push-out $\Delta G_{e}$ functionally distinguishes an electrostatically (b) from a chemically (c) generated level offset. 
(see Sec. 4.3 and 4.4) [192]. Fig. 6.3a shows that the electron states on minority acceptor molecules (those molecules that form part of the protrusion) adopt the electrostatic character of the majority donor domain: They are acceptor states with a donor electrostatic dressing and as such experience a reduced stabilization. The reduction amounts to around $0.2 \mathrm{eV}$ : This almost matches the total CT binding energy for this system. The total binding energy, however, consists of multiple smaller contributions, which are associated with individual hopping events that, executed in succession, transform a CT into a CS state; in other words, the competition between Coulomb attraction and dielectric solvation locally leads to smaller barriers for separation. The largest of these local hopping barriers occur during the conversion from a nearest-neighbour electron-hole pair to a next-nearest and next-next-nearest-neighbour pair. Specifically, the first two hopping barriers together amount to only $0.2 \mathrm{eV}$. The $0.3 \mathrm{eV}$ can be harvested for these first two and most crucial steps in the separation process, which as a result is locally driven.

In spite of its model character, this analysis already shows that mesoscale fields can be used to generate a stepped energy cascade that locally drives the charge splitting process [193]. The cascade is in this case an intrinsic property, rather than the result of a tailored interlayer comprising a third molecular species $[39,194]$. The homogeneity of the mesoscale fields also implies an important functional difference between an electrostatic and a purely chemical level offset, pointed to in Fig. 6.3b-c: Only the electrostatic offset (Fig. 6.3b) provides driving forces $\Delta G$ for minority electron and hole push-out,

$$
\begin{aligned}
& \Delta G_{e}=\left.\Delta_{e(A)}\right|_{D}-\left.\Delta_{e(A)}\right|_{A}, \\
& \Delta G_{h}=\left.\Delta_{h(D)}\right|_{A}-\left.\Delta_{h(D)}\right|_{D} .
\end{aligned}
$$

Here, $\left.\Delta_{e(A)}\right|_{D}$ denotes the environment contribution to the electron $(e)$ state energy of the acceptor $(A)$ embedded in the donor $(D)$ domain. Definitions of the other $\Delta$ 's follow by analogy. If donor and acceptor are electrostatically identical, $\Delta G_{e}=\Delta G_{h}=0$; the total level offsets are then given simply by the respective chemical offsets, i.e., the difference in gas-phase IEs and EAs. Such a chemical offset (Fig. 6.3b) may assist in generating CT states, but it cannot drive the CT separation process.

Comparing the cartoon from Fig. 6.3b to the atomistic model from Fig. 6.3a, we note that the atomistic morphology suffers from an important shortcoming: Only a tiny fraction of donor-acceptor pairs, specifically those pairs whose acceptor unit resides in the small fullerene appendix, experiences the push-out forces from Eq. 6.5. The large majority of interfacial electron-hole pairs, however, will be subjected to the full Coulomb barrier, and charge separation has to occur without the assistance of mesoscale fields. This picture is very different from the (at this point still purely conceptual) schematic in Fig. 6.3b, where indeed hole-electron pairs over the entire interfacial area benefit from these driving forces. 


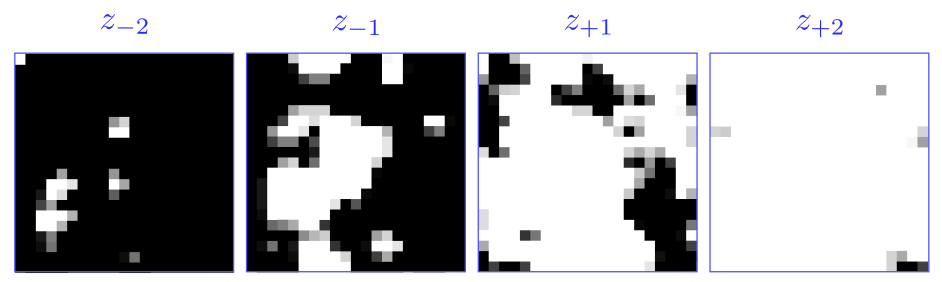

Figure 6.4. Composition maps for an interfacial lattice morphology with interface roughness. Cross sections through the donor-acceptor interphase at $z_{-2}=-0.8 \mathrm{~nm}$ (left) to $z_{+2}=+0.8 \mathrm{~nm}$ (right). Donor (acceptor) regions are coloured white (black). At $z_{-1}$ and $z_{+1}$, the donor/acceptor ratio is $30 \%: 70 \%$ and $70 \%: 30 \%$, respectively.

\subsubsection{Charge Push-Out Forces at Rough Interfaces}

A realistic model system should hence account for both donor-acceptor interpenetration on a domain scale and intermixing on a molecular scale. This is rather tricky to achieve on an atomistic level, as simulations would not only have to address very large system sizes, but also appropriately sample the free energy landscape. As a further downside of small atomistic models with imposed thermal disorder, finite-size-induced fluctuations of layer dipoles and quadrupoles limit a meaningful comparison of donor and acceptor energy levels to the vicinity of the interface (as depicted in Fig. 6.3a). We therefore switch to a lattice model, where each lattice site represents either a donor or acceptor molecule. For parametrization, reference molecules (here D5M and $\mathrm{C}_{60}$ ) are electrostatically coarse-grained into multipolar polarizable lattice sites via Eq. 3.77. To arrive at a more generic model, the multipole expansion of the D5M lattice site is furthermore reduced to the quadrupolar moment $Q_{20}$ associated with the long molecular axis. Note that - as the lattice model preserves the molecular quadrupole moment per volume - long-range interactions can be quantitatively accounted for.

The cubic lattice incorporates 8000 molecular sites spaced with a lattice constant of $0.55 \mathrm{~nm}$. Metropolis Monte-Carlo sampling is used to equilibrate the interfacial morphology, starting from a clean interface with the acceptor (donor) domain located in the half-space $z<0(z>0)$, and the particle type of the bottom-most and top-most layers constrained. Pairwise nearest-neighbour interaction energies for donor-donor $\left(\varepsilon_{D D}\right)$, donor-acceptor $\left(\varepsilon_{D A}\right)$, and acceptor-acceptor $\left(\varepsilon_{A A}\right)$ contacts were chosen as $\varepsilon_{A A}=\varepsilon_{D D}$ and $\varepsilon_{D A}-\varepsilon_{D D}=k_{\mathrm{B}} T$. With these interaction parameters, the interphase between donor and acceptor comprises approximately two monolayers of composition 30\%D:70\%A and 70\%D:30\%A. The morphology is characterized by domain interpenetration rather than fine intermixing, as indicated by composition maps of the four monolayers closest to the interface, shown in Fig. 6.4. The phenomenological contact interactions lead to the overall concentration profile plotted in Fig. 6.5c.

To assess the contribution of mesoscale fields to the charge separation process, we define charge push-out fields $f_{h(D)}(i, j)$ for hole transfer between nearest-neighbour donor sites $i$ and 

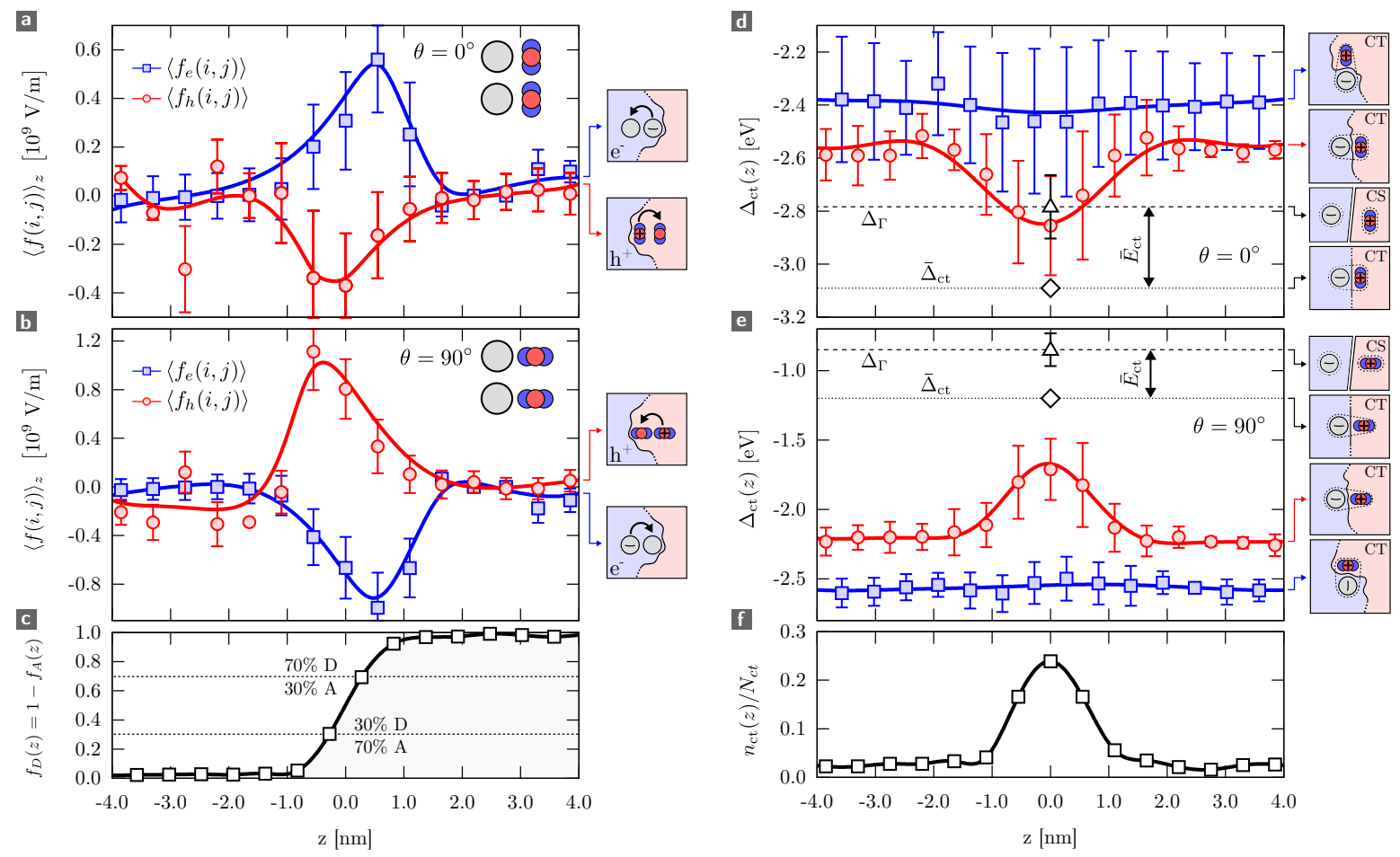

Figure 6.5. Charge push-out across a donor-acceptor interphase via mesoscale fields. Interfacial layer-averaged fields $\left\langle f_{h(D)}(i, j)\right\rangle_{z}$ and $\left\langle f_{e(A)}(i, j)\right\rangle_{z}$ (for definition, see text) across a heterojunction between quadrupolar polarizable donor sites and apolar polarizable acceptor sites in an (a) face-on and (b) tip-on orientation. The donor sites mimic an ADAtype compound with long-axis quadrupole moment $Q_{20}<0$. The push-out fields are narrowly peaked over an interfacial region with the donor concentration profile given in (c). Polarity and magnitude of these fields impacts the energetics of CT states as again illustrated for the (d) face-on and (e) tip-on case, where $\Delta_{\text {ct }}$ (electrostatic and polarization contribution to CT-state energies) is shown for pairs formed between adjacent layers (red line with circles) and within the same layer (blue line with squares). The resulting $\Delta_{\mathrm{ct}}$ should be compared to the case of a sharp interface ( $\bar{\Delta}_{\mathrm{ct}}$, dotted line with diamond) as well as to the correction to the photovoltaic gap $\Delta_{\Gamma}$. With most pairs found in the interphase region, as indicated by the pair concentration profile (f), mesoscale fields shift the CT state energy for the face-on scenario into the photovoltaic gap, leading to unbound CT states. CT states in the tip-on scenario (e) experience the opposite effect: $\Delta_{\text {ct }}$ moves further away from $\Delta_{\Gamma}$, leading to higher CT binding energies. 
$j$, and $f_{e(A)}(i, j)$ for electron transfer between nearest-neighbour acceptor sites:

$$
\begin{aligned}
f_{h(D)}(i, j) & =\frac{\Delta_{h(D)}(j)-\Delta_{h(D)}(i)}{q\left(z_{j}-z_{i}\right)}, \\
f_{e(A)}(i, j) & =\frac{\Delta_{e(A)}(j)-\Delta_{e(A)}(i)}{q\left(z_{j}-z_{i}\right)} .
\end{aligned}
$$

Here, $z_{i}$ and $z_{j}$ are the positions of sites $i$ and $j$, respectively, measured along the interface normal. A positive $f$ implies a driving force directed towards the acceptor domain, irrespective of the sign of the charge $q= \pm 1$ e. The gross driving force from Eq. 6.5 can be formally recovered by integration over the layer-averaged pairwise driving fields $\left\langle f_{h(D)}(i, j)\right\rangle_{z}$ and $\left\langle f_{e(A)}(i, j)\right\rangle_{z}$, for example $\Delta G_{h(D)}=-q \int\left\langle f_{h(D)}(i, j)\right\rangle_{z} \mathrm{~d} z$. These layer-averaged fields are plotted in Fig. 6.5a-b as a function of the position along the interface normal. They have been calculated for two different molecular orientations of the donor site, face-on (Fig. 6.5a) and tip-on (Fig. 6.5b). Only the face-on configuration, however, yields the correct polarity of the interphase driving fields, pushing holes towards the donor, and electrons towards the acceptor. In the tip-on orientation, the polarity is reversed: Trapping of charge carriers on minority sites is in this case likely. A solar cell built around the tip-on orientation is hence expected to suffer from trap-assisted recombination, here: recombination of a majority carrier with a trapped minority carrier.

We now investigate how the push-out fields impact the energy landscape for CT states. For reference, we first consider a flat interface, with all donor sites located in $z>0$, acceptor sites in $z<0$. We denote the electrostatic and induction contributions to the CT state energy as $\bar{\Delta}_{\text {ct }}$ and the corresponding photovoltaic gap as $\bar{\Delta}_{\Gamma}$, where the bar relates to the flat interface. Both $\bar{\Delta}_{\Gamma}$ (dashed line with triangle) and $\bar{\Delta}_{\text {ct }}$ (dotted line with diamond) are shown in Fig. 6.5d-e for the face-on (d) and tip-on (e) molecular orientations. In both cases, the CT binding energy, $\bar{E}_{\text {ct }}=\bar{\Delta}_{\Gamma}-\bar{\Delta}_{\text {ct }}$, amounts to the usual $0.3 \mathrm{eV}$. Note that the lattice description employs a damped intra-pair charge-charge interaction (whose magnitude is overestimated due to the coarse-grained description) that recovers a typical electrostatic (i.e., first-order) electron-hole attraction energy of $-1.6 \mathrm{eV}$, as for example seen in $\mathrm{C}_{60} / \mathrm{D} 5 \mathrm{M}$.

For the system with finite interface roughness, the concentration profile of molecular pairs forming CT states is shown in Fig. 6.5f. First, we note that this roughness, which leads to a broadened peak in the concentration profile, leaves the solid-state contribution to the photovoltaic gap unaffected, $\Delta_{\Gamma}=\bar{\Delta}_{\Gamma}=\left.\Delta_{e(A)}\right|_{A}+\left.\Delta_{e(D)}\right|_{D}$, as $\Gamma$ only probes states located well within the donor and acceptor films. $\Delta_{\text {ct }}$, however, proves rather sensitive to the degree of intermixing. Here, we distinguish between two types of CT states, either formed between two adjacent layers (red line with circles), or within the same layer (blue line with squares), as depicted on the right-hand side of Fig. 6.5d-e. For the face-on configuration (Fig. 6.5d), energies of $\mathrm{CT}$ states formed between two adjacent layers are shifted up by $0.3-0.5 \mathrm{eV}$ compared to $\bar{\Delta}_{\mathrm{ct}}$, even surpassing $\Delta_{\Gamma}$ away from the interface $(|z| \geq 0.6)$, leading to a negative $E_{\mathrm{ct}}$, and hence unbound CT states. 
One should of course consider that CT states far away from the interface are absent for flat, and rare for rough interfaces, with the vast majority of the CT states found across a narrow interphase between $z=-1 \mathrm{~nm}$ and $z=+1 \mathrm{~nm}$ (see the pair concentration profile in Fig. 6.5f). Across this interphase, charge push-out fields are particularly strong, and yet, $\Delta_{\mathrm{ct}}$ in fact experiences a dip at around $z=0$, as the high-energy minority hole and low-energy majority electron states in the acceptor domain $(z<0)$ are traded for high-energy minority electron and low-energy majority hole states in the donor domain $(z>0)$. Still, the mean binding energy in the $z=0$ slice is only on the order of $0.05 \mathrm{eV}$, which should be thermally accessible.

The second type of CT state, with both charges located within the same layer, is subjected to an even larger total push-out force, such that the dip at $z=0$ disappears (blue line in Fig. 6.5d). At the same time, these CT states are shifted upwards by an additional $0.2 \mathrm{eV}$ due the anisotropy of the quadrupolar molecular species. In the case of $\mathrm{C}_{60} / \mathrm{D} 5 \mathrm{M}$, for example, the in-plane CT states correspond to a tip-on pair with a larger separation between hole and electron, leading to a reduced electronic coupling.

For the alignment of the negative quadrupole moment with the interface normal (Fig. 6.5e), the scenario is reversed due to the opposite (and unfavourable) polarity of the push-out forces. Indeed, the CT binding energy is now increased by $0.3-1.0 \mathrm{eV}$. Depending on the internal energy landscape, CT states will then either dissociate in the wrong direction (effectively reversing the role of donor and acceptor) or simply remain stationary, until recombination occurs.

As a conclusion of the above, suitably tailored interfacial push-out forces can indeed shift CT state energies into the photovoltaic gap, leading to unbound or weakly bound states. Correct polarity and magnitude of the forces depend on both molecular orientation and intermixing, with the latter giving rise to nanoscale surface roughness. So what happens upon varying the degree of this roughness? First off, for a fixed interfacial orientation, the gross driving forces $\Delta G_{e}(A)$ and $\Delta G_{h}(D)$ do not change with the degree of donor-acceptor intermixing. Consequently, as the size of the interphase grows, the area below the curves in Fig. 6.3 is conserved, whereas the peak heights decrease, and the peak widths increase. This implies a tradeoff between interphase size and magnitude of the charge push-out fields: If the interphase is too narrow, we have a scenario as seen for the atomistic D5M/C 60 model, where pair splitting fields are sizeable, but the fraction of interfacial sites that can harvest these fields is small. If the interphase is wide, the fraction of interfacial sites that are subjected to the pair splitting fields will be considerable, but the fields themselves may be insufficient to overcome $E_{\text {ct }}$. For a given donor-acceptor combination, the optimum degree of intermixing will hence depend on the relative magnitudes of $E_{\mathrm{ct}}$ on the one hand, and $\Delta G_{e}$ and $\Delta G_{h}$ on the other hand. 

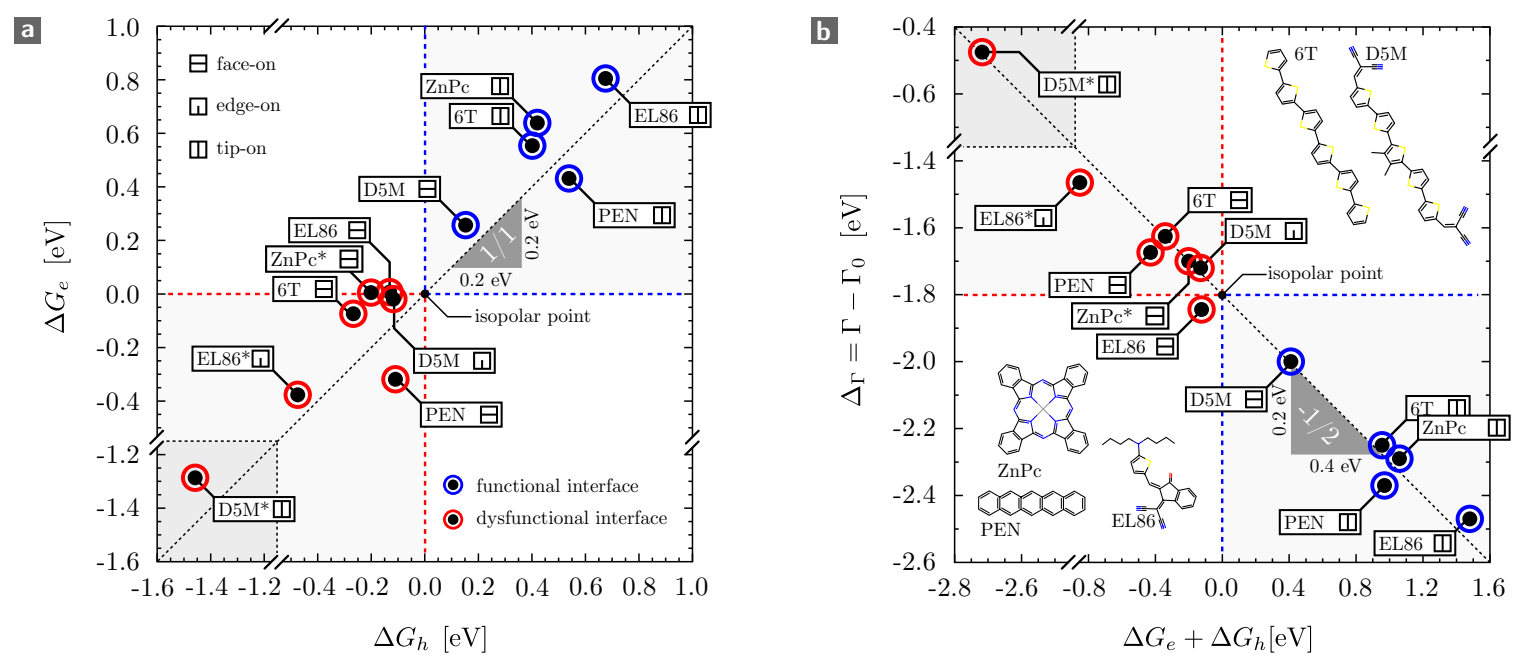

Figure 6.6. Tradeoff between charge push-out and photovoltaic gap. (a) Correlation plot of driving forces $\Delta G_{h}$ and $\Delta G_{h}$ computed for five donor materials in combination with $\mathrm{C}_{60}$, incorporating up to three interfacial orientations (face-on, tip-on, edge-on). Configurations found experimentally for the respective planar heterojunction are circled in blue. Configurations circled in red are expected to yield dysfunctional cells. (b) Tradeoff between change in photovoltaic gap $\Delta_{\Gamma}=\Gamma-\Gamma_{0}$ versus total charge push-out force $\Delta G_{h}+\Delta G_{e}$. Calculations are performed on $10 \mathrm{~nm}$ donor films on top of $10 \mathrm{~nm} \mathrm{C}_{60}$ or (for compounds marked with an asterisk) $10 \mathrm{~nm}$ dielectric with $\varepsilon \simeq 4$, shown to yield transferable results.

\subsection{Interface Polarity versus Photovoltaic Gap}

We have by now established that mesoscale order generates orientation-dependent, homogeneous charge push-out fields across a donor-acceptor interphase. The upper bound of these fields is set by the driving forces $\Delta G_{e(A)}$ and $\Delta G_{h(D)}$ that result from the difference in electrostatic and polarization contributions to site energies in the pristine donor and acceptor films. Poled correctly, they locally drive the charge separation process, provided they surpass the local Coulomb barriers that sum up to the total CT binding energy $\bar{E}_{\text {ct }}$ defined in the absence of any charge push-out fields. This binding energy is reasonably constant across different donor-acceptor materials, as a consequence of the similar dielectric properties that through dielectric solvation place $\bar{E}_{\text {ct }}$ around $0.3 \mathrm{eV}$.

The question arises: Which magnitudes of $\Delta G_{e(A)}$ and $\Delta G_{h(D)}$ are physically realizable (and realized) in organic solar cells to oppose this nevertheless strong attraction of the electron-hole pair, in particular in small-molecular systems? To address this, we have investigated five different donor materials used in combination with $\mathrm{C}_{60}$ as acceptor: pentacene (PEN), sexithiophene (6T), zinc-phthalocyanine ( $\mathrm{ZnPc})$, the merocyanine dye EL86 and acceptor-substituted oligothiophene D5M. Model interfaces were assembled from the crystal structures of the respective components: The $\mathrm{C}_{60}$ exposes its $f c c$ [111] surface to the donor, whose X-ray crystal structures we cleaved to obtain the desired orientation on the $\mathrm{C}_{60}$ substrate. The mismatch between the crystal faces was corrected by equilibration of the $\mathrm{C}_{60}$ 
using molecular dynamics.

Due to the orientation dependence, clearly no unique value for $\Delta G$ can be assigned to any given donor-acceptor combination. We have therefore incorporated up to three packing modes per donor, corresponding to face-on, edge-on and tip-on molecular orientations. A summary of the computed charge push-out forces is provided in Fig. 6.6a, which illustrates the expected linear correlation between $\Delta G_{h}$ and $\Delta G_{e}$. The variation of the $\Delta G$ 's with orientation can easily exceed $1 \mathrm{eV}$, as is observed for the strongly polar donor-acceptor- and acceptordonor-acceptor-type materials EL86 and D5M, respectively (see also Sec. 4.3). Strikingly, the experimentally reported configurations in the respective planar heterojunction solar cell are all located in the first quadrant, where $\Delta G_{e}, \Delta G_{h}>0$ : This condition appears to be a prerequisite for functional solar cells. As has already been discussed in the context of the lattice model (Fig. 6.5), there is a clear rationale why cells with negative charge push-out forces should suffer from inefficient charge generation and extraction: With $\Delta G_{e}, \Delta G_{h}<0$, charges are pushed out from the interphase in the wrong direction and hence trapped on minority sites. Correctly poled, the mesoscale fields therefore come with a dual benefit - they assist both charge splitting and minority-carrier detrapping. Of these two effects, the former is complimentary, the latter essential. Indeed, relating back to Fig. 6.1, the need for minoritycarrier detrapping (and extraction) finally explains why only the tip-on configuration of EL86 on $\mathrm{C}_{60}$ is expected to work well without suffering from extensive recombination across the entire donor-acceptor interface.

The isopolar point, defined by $\Delta G_{e}=\Delta G_{h}=0$, hence marks the border point between a dysfunctional and functional interface. This said, large positive $\Delta G$ 's appear most desirable, but they come at the cost of a reduced photovoltaic gap (which linearly correlates with the CT-state energy and hence $V_{\mathrm{oc}}$ ): To illustrate this, Fig. 6.6b correlates the sum of $\Delta G_{e}$ and $\Delta G_{h}$ against the difference $\Delta_{\Gamma}=\Gamma-\Gamma_{0}$ in the photovoltaic gap evaluated in gas-phase $\left(\Gamma_{0}\right)$ and in the solid state $(\Gamma)$. The tradeoff between $\Delta_{\Gamma}$ versus $\Delta G_{e}+\Delta G_{h}$ occurs at a rate of $-\frac{1}{2}$, since the acceptor IE and donor EA can be modified without impacting $\Gamma$. The largest reduction in $\Gamma$ follows for the solar-cell configuration of EL86, in line with a sizeable total push-out force of almost $1.5 \mathrm{eV}$. A push-out force on the order of the CT binding energy should, however, suffice to efficiently generate free charges. With the CT binding energy located at $0.3 \mathrm{eV}$, much of the $1.5 \mathrm{eV}$ are hence wasted - even though a driving force of this magnitude may still be necessary in the case of strong donor-acceptor intermixing (as explained in Sec. 6.1.3). Otherwise they result in uncalled-for structural $V_{\text {oc }}$ losses. Assuming perfect morphological control, a compromise between $\Gamma$ and charge push-out forces is therefore obtained for operation just above the isopolar point marked in Fig. 6.6a. It has in fact been realized for the face-on configuration of D5M, which balances driving forces for charge pushout and detrapping with moderate gap-related $V_{\text {oc }}$ losses. This loss in $\Delta_{\Gamma}$ (associated with the position of the mean of the DOS) is furthermore accompanied by a disorder-related impact on $V_{\text {oc }}$ (associated with the tail of the DOS). D5M, for example, achieves an exemplary compromise for the former, but performs rather poorly regarding the latter, due to sizeable energetic disorder of $0.1 \mathrm{eV}$ - the largest among all compounds studied here. 


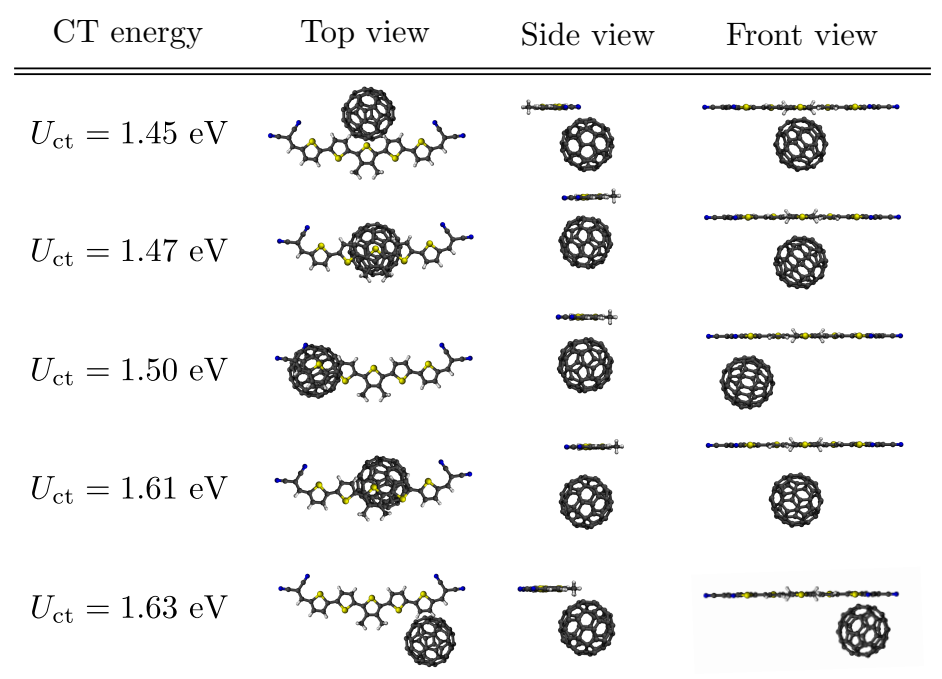

Table 6.1. Spread of CT state energies among dimer configurations. The three right-hand columns portray five representative donor-acceptor configurations encountered at the $\mathrm{C}_{60} / \mathrm{D} 5 \mathrm{M}$ interface. The $\mathrm{CT}$ state energies $U_{\text {ct }}$ listed in the left column were computed via the long-range polarized embedding approach (Chapter 3). In the computation, the molecular environment (though not shown in the dimer visuals) was explicitly accounted for.

Last, but not least, the tradeoffs identified above also hold for non-fullerene systems: A polar acceptor unit would, however, impose stricter orientational constraints, as an unfavourable acceptor orientation could then pin the interface to a negative $\Delta G$ whatever the orientation of the donor. Independent of the type of acceptor used, molecular orientations with a negative $\Delta G$ and as a result enlarged $\Gamma$ may also prevent Frenkel- to CT exciton conversion - another reason, why solar cells working in that regime may have deficiencies. In a similar way, the need to generate CT states from Frenkel excitons sets an upper limit for how much the CT-state energy may be raised through intermixing in configurations with $\Delta G>0$.

\subsection{Charge Transfer Energetics and Open-Circuit Voltage}

In the final part of this chapter on charge transfer energetics, we will characterize charge transfer states in different $\mathrm{C}_{60}$ /donor systems, previously studied with regard to chargecarrier level profiles (Sec. 4.3) and interface polarity (Sec. 6.2 above). In particular, we will explore relationships among the densities of states of charge carriers and CT states and the open-circuit voltage - taking into account charge density and temperature.

When computing the distribution of CT energies, we limit our study to atomistic models with ideal interfaces. Due to positional disorder that follows from molecular mismatch at the donor-acceptor interface, these systems already sample more than just one CT state energy. As an example, Table 6.1 summarizes five different pair configurations encountered at the $\mathrm{C}_{60} / \mathrm{D} 5 \mathrm{M}$ interface. The energy of the CT state, $U_{\mathrm{ct}}$, is listed in the left column. These five 
pairs already display a large spread of $\mathrm{CT}$ energies of close to $0.2 \mathrm{eV}$ as a consequence of the different in-plane alignment of the dimers. To adequately sample this type of positional disorder, we consider up to 100 of the closest pairs per system. The final set is, however, subject to the in-plane system dimensions required to obtain an $x y$-periodic interface within the crystal patching approach. Hence, in cases where the crystal structures match well, fewer pairs can be studied due to the smaller simulation cell.

Fig. 6.7 presents a summary of the photovoltaic gap (red bars), Frenkel exciton (FE) energy (green bars) and charge transfer energies (blue bars) as extracted from simulations. The FE calculations were performed in gas-phase using GW-DFT (a many-body Green's function approach) within the Bethe-Salpeter formalism [195]. The stabilization of FEs in the solid state is typically small, amounting to $0.2 \mathrm{eV}$ for D5M. The experimental open-circuit voltage is indicated by black bars. Yellow bars denote the mean CT energy, to be interpreted with care due to the preselection of $\mathrm{CT}$ configurations. As before, we take into account different donor orientations on the $\mathrm{C}_{60}$ substrate. The $\mathrm{CT}$ levels of the respective higherlying configuration are coloured grey for clarity. First, inspection of CT binding energies, taken as the difference between photovoltaic gap and mean CT energy, suggests $0.2 \mathrm{eV}$ for tip-on, $0.3 \mathrm{eV}$ for edge-on and $0.4 \mathrm{eV}$ for face-on orientations as a rule of thumb. These values are within our estimate for the dielectric-solvation regime in Sec. 6.1.

With regard to exciton levels, it is perhaps not surprising that the energy separation between the CT band and FE energy is smallest for the world-record material D5M, thought advantageous in that a smaller CT-FE gap increases the thermodynamic efficiency, as long as FE-to-CT conversion is not hindered. Furthermore, and interestingly, the lower edges of the CT bands are located $0.4-0.5 \mathrm{eV}$ above the experimental $V_{\text {oc }}$ for the low-lying energy configurations. This $E_{\mathrm{ct}}-V_{\mathrm{oc}}$ gap is remarkably constant across the different materials, and consolidated by optical measurements, which place the gap at around $0.5-0.6 \mathrm{eV}$, with the lower value associated with planar heterojunctions by trend (see the work by K. Vandewal [196]). The apparent generality of this correlation, here derived with simulated CT energies, can be related to a generic steady-state charge density at open-circuit conditions, used in Sec. 4.3 to estimate the open-circuit voltage from microscopic simulations. For the low-energy configurations shown in Fig. 6.7, the difference between photovoltaic gap and open-circuit voltage can hence be excellently accounted for through population of the thermally broadened DOS at a charge-carrier density of $10^{16} \mathrm{~cm}^{-3}$.

Further insights into the role of charge transfer states can be gained from temperaturedependent simulations and measurements of the open-circuit voltage. We limit this analysis to two compounds, D5M and EL86. First, we compute the temperature dependence of the DOS of charge carriers. To this end, molecular dynamics simulations are performed in the $0-300 \mathrm{~K}$ temperature range, starting from larger supercells at $0 \mathrm{~K}$, then proceeding with the thermalization of the structures (see also Fig. 2.5). Fig. 6.8 reports the temperature-dependent thermal broadening $\sigma_{h}(T)$ of the DOS of holes (red curves) in D5M (Fig. 6.8a) and EL86 (Fig. 6.8b), computed from the equilibrated morphologies. With the mean of the DOS extracted from calculations on thin films (Fig. 6.7), the iso-density open-circuit voltage for these 


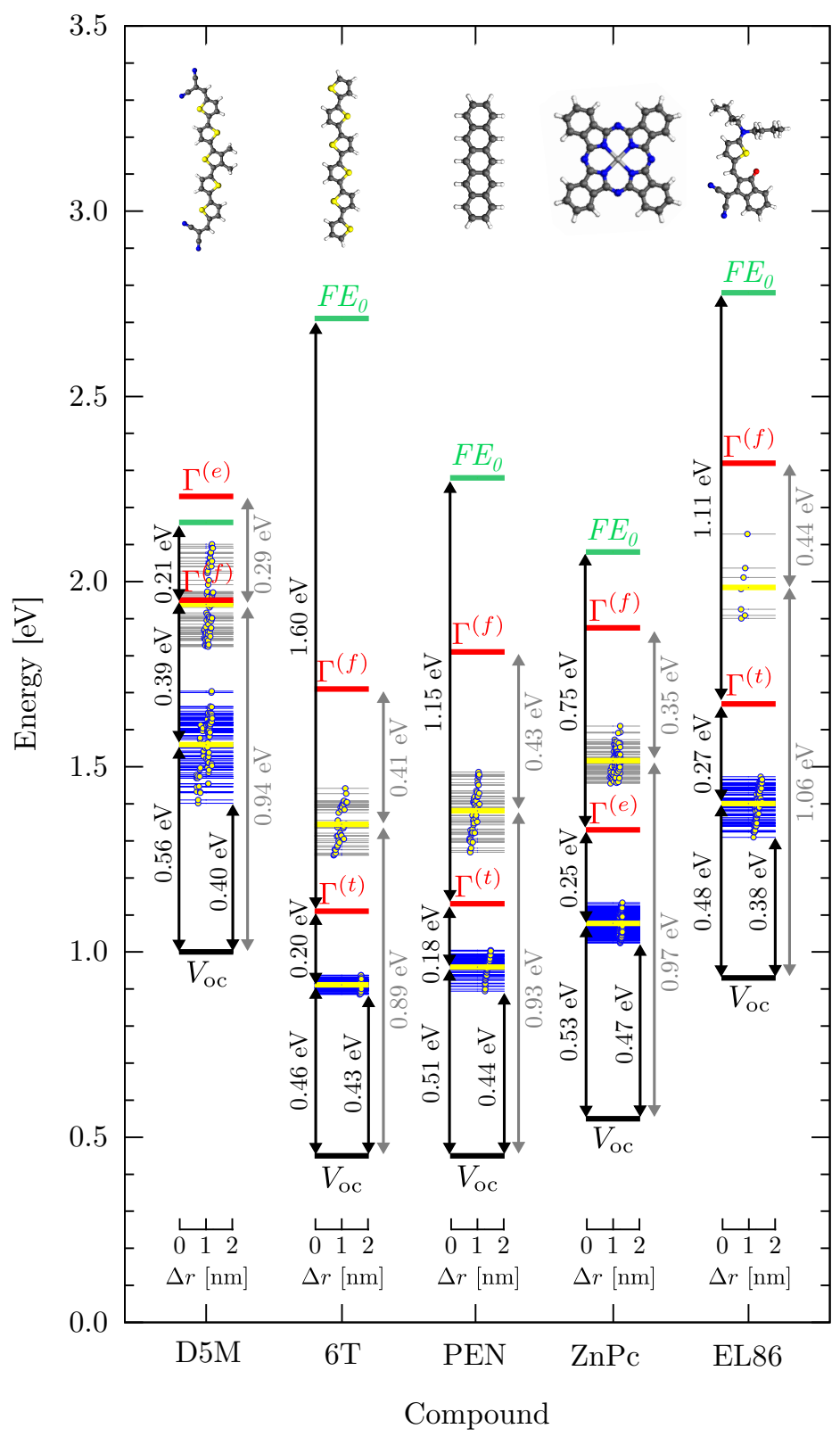

Figure 6.7. Jablonski-type device diagram of planar heterojunction solar cells. Simulated photovoltaic gap (red bars), charge transfer state energies (blue bars for lowest-energy configurations, otherwise grey), gas-phase Frenkel exciton energies (green bars) and experimental open-circuit voltage (black bars) for five $\mathrm{C}_{60} /$ donor combinations. The different donor orientations on the $\mathrm{C}_{60}$ substrate are indexed by $f$ (face-on), $t$ (tip-on) and $e$ (edge-on). The inset distance scales indicate the donor-acceptor separation in the charge transfer dimers, as marked for each charge transfer state by means of the yellow dot. Yellow bars indicate the mean charge transfer state energy established from the preselected dimers. 

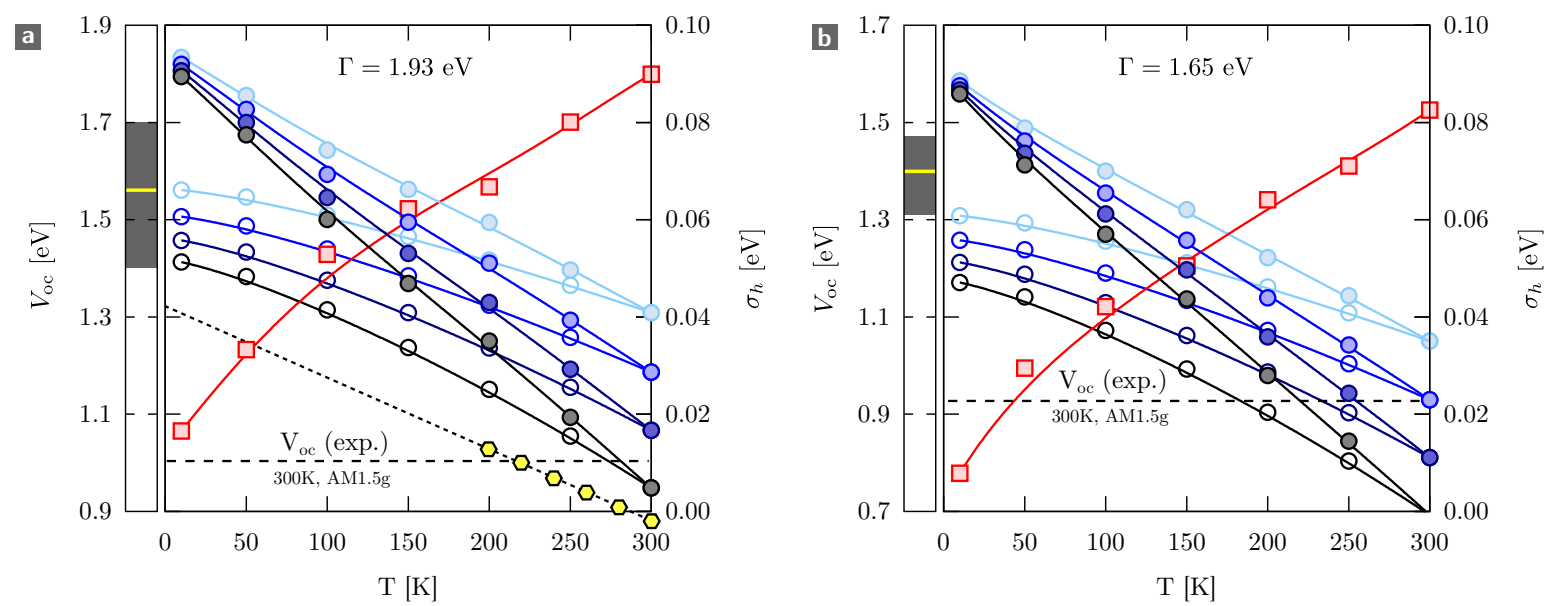

Figure 6.8. Temperature-dependent $\boldsymbol{V}_{\mathrm{oc}}$ in D5M and EL86. Simulated ("canonical") open-circuit voltage $V_{\text {oc }}(p, T)$ with (blue curves with filled circles) and without (blue curves with open circles) inclusion of the temperature-dependent disorder $\sigma_{h}(T)$ (red curve with squares) obtained from thermalized supercells in D5M (a) and EL86 (b). For $V_{\text {oc }}(p, T)$, the upper (light-blue) trace corresponds to a hole occupation fraction $p=10^{-4}$, the lower (black) trace to $p=10^{-7}$; for the middle traces, $p=10^{-5}$ and $p=10^{-6}$. The black bars in the left columns of either graph mark the CT band as previously shown in Fig. 6.7. In (a), the dashed line with yellow symbols denotes the experimentally measured ("grand-canonical") voltage trend $V_{\mathrm{oc}}(T)$ measured for a $\mathrm{C}_{60}$ :D5M bulk heterojunction by our collaborator J. Widmer (University of Dresden). 
systems follows from Eq. 4.8: This voltage, $V_{\mathrm{oc}}(p, T)$, is shown for donor occupation fractions of $p=10^{-7}$ (black curve with solid circles) to $p=10^{-4}$ (light blue curve with solid circles) in decadic steps. The voltage trends intersect at $T=0$ at the photovoltaic gap $\Gamma$. Their slope is linearly dependent on $\log (p)$ with a positive prefactor $\alpha$ :

$$
V_{\mathrm{oc}}(p, T)=\Gamma-\alpha \log (1 / p) k_{\mathrm{B}} T
$$

We note that this form of $V_{\mathrm{oc}}(p, T)$ is a consequence of the thermal narrowing of the DOS towards lower temperatures; indeed, for constant $\sigma_{h}$, a rather different behaviour results (black to blue curves with open circles). In the next step, we compare $V_{\text {oc }}(p, T)$ and the distribution of $\mathrm{CT}$ energies (indicated by the black bar in the left panel of Fig. 6.8a) to the experimentally determined voltage trend $V_{\text {oc }}(T)$ obtained for a $\mathrm{C}_{60}$ :D5M bulk heterojunction (dashed line with yellow circles). In this comparison, we should furthermore consider that for this system, the open-circuit voltage in a planar heterojunction setup turns out approximately $0.1 \mathrm{eV}$ larger than in the bulk setup. Extrapolating the experimental trend towards $T=0$, we obtain a zero-temperature $V_{\text {oc }}$ that coincides well with the lower edge of the computed CT band at $1.4 \mathrm{eV}$. In a classical picture, this can be understood in that at zero temperature, i.e., in the absence of thermal activation, the open-circuit voltage is pinned by the CT state energy. Based on these simulated and experimental voltage trends, charge generation at open-circuit conditions has to follow an activation law, with the iso-density voltage $V(p, T)$ from Eq. 6.7 and the experimentally measured $V(T)$,

$$
V_{\mathrm{oc}}(T)=U_{\mathrm{ct}}-\alpha^{\prime} k_{\mathrm{B}} T
$$

From intersecting the two trends, we can determine the temperature-dependent steady-state charge density $p^{*}$ as

$$
p^{*} \sim \exp \left[-\frac{1}{\alpha} \frac{\left(\Gamma-U_{\mathrm{ct}}\right)}{k_{\mathrm{B}} T}\right] .
$$

In this expression, the prefactor $\alpha$ captures field-enhancement or -reduction of charge generation and thus also incorporates morphological features. Eq. 6.9 finally indicates that the charge separation process even in highly efficient solar cells may still rely on thermal activation - and thus involve CT states with a finite positive binding energy.

\subsection{Summary: Charge Transfer Energetics}

We demonstrated that mesoscale fields provide charge push-out and detrapping forces that can assist in the initial phase of electron-hole-pair separation. These driving forces result from the coherent superposition of quadrupolar fields that accompany long-range molecular ordering in a thin-film setup. Due to their mesoscopic origin, they are characterized by pronounced inplane homogeneity and an out-of-plane discontinuity. In the presence of mild donor-acceptor 
intermixing, they are sharply peaked over a narrow interphase and locally drive the chargeseparation process if dimensioned and poled correctly, pointing to the functional difference between chemically and electrostatically generated level offsets.

Correct polarity of the driving forces serves as a prerequisite for efficient solar-cell operation, as is suggested by the comparison of different donor materials and interfacial configurations. This observation indicates that the action of homogeneous push-out forces accounts for a pathway for charge separation that could not be realized with only charge delocalization [197] or energetic disorder [134]. Presence of these fields rationalizes why cold excitons [186] can suffice to obtain free charges - and, most importantly, why separation can be barrierless [187]. If, however, they only barely compensate the exciton binding energy, hot states [188, 198] may nevertheless prove helpful. Still, it remains to be seen, to which extent the mechanism of charge push-out is already built into today's organic solar cells, considering also that the CT-state energy should not be raised above the energy of a Frenkel exciton.

The link between driving-force polarity and molecular orientation and intermixing suggests morphological boundary conditions for efficient charge splitting. As a rule, there is a 2:1 tradeoff between charge push-out forces versus photovoltaic gap, with operation closely above an isopolar point providing an energetic compromise. We note, however, that this compromise only accounts for the structural factors that feature in the open-circuit voltage. Object of further study is hence to understand how the complex energy landscape for CT states that is suggested by calculations on interfaces with realistic patterning impacts dynamic factors that determine the steady-state charge density. Either way, the sensitivity of charge push-out forces to the degree of nanoscale surface roughness and molecular orientation, as well as the implied tradeoffs for cell energetics, further illustrate why material choice and processing are such a formidable challenge for device fabrication. 


\section{Chapter 7}

\section{Conclusions \& Outlook}

I have investigated the diverse ways in which molecular and morphological features on the microscopic and mesoscopic scale jointly condition the density of states of charge carriers and charge transfer states in organic semiconductors. Using particle-based descriptions with atomistic or coarse-grained resolution, the underlying computational framework has enabled quantitative insight into the energetics of organic heterostructures, grain boundaries, partially ordered polymeric and amorphous mesophases relevant in organic solar cells, light-emitting diodes and transistors. Long-range interactions in particular have proven fundamental to the study of organic semiconductors, relating the energetics on a molecular and supramolecular level to device-level characteristics. In this final chapter, I will retrace the derived structureproperty relationships and design rules for organic electronic devices, outlining challenges in the understanding and modelling of organic semiconductors.

Impact of Mesoscale Order at Organic-Organic Interfaces The functionality of organic solar cells relies on suitably tailored energy level profiles with appropriate interfacial offsets and bending that ideally favour charge generation and prevent recombination. The qualitative features of the resulting profiles were, however, subject of discussion, as computational studies strived to understand the functionality of highly efficient devices. We could show how microscopic simulations successfully access these energy profiles if mesoscopic ordering, probed by long-range electrostatic interactions, is properly accounted for. The resulting level profiles are thus subject to an order criterion: Flat level profiles across thin films - as a frequent experimental finding - only result for pronounced in-plane structural coherence over a length scale that well exceeds the thickness of the film. This link to the mesoscopic organization in crystalline thin films is an intriguing consequence of the quadrupolar structure shared by the vast majority of organic semiconductors. The action of the associated longrange fields sets this class of materials apart from disordered soft matter on the one hand, which lacks the structural coherence to generate these fields, and inorganic semiconductors on the other hand, which exhibit strong screening mechanisms that compensate them. 
Role of Molecular Architecture, Packing and Orientation From a comparative study of various small-molecular materials, we could show how molecular architecture and orientation control the energy level alignment across thin films. The molecular architecture, which can be of a donor, donor-acceptor, or acceptor-donor-acceptor type, establishes an energy hierarchy for the face-on, edge-on and tip-on orientations. The computational approach captures both the absolute and relative change of level positioning upon reorientation of the molecular crystals with excellent accuracy, as seen from the comparison with photoemission spectra. Meanwhile, molecular packing into, for example, brickwork vs. herringbone structures, was found to be less relevant in thin films, as the energetics are then determined by the competition between in-plane and out-of-plane interaction directions. The polarity of the latter is only weakly influenced by crystalline repacking. Previous studies of bulk energetics, by contrast, have attributed a stronger role to crystalline packing modes. For bulk (i.e., 3Dinfinite) systems, where orientation is of course undefined, this should indeed be the case. The good agreement between thin-film levels and experimentally determined energies indicates, however, that a bulk description should by no means be used to assess out-of-plane energy level alignment. We have nevertheless found molecular packing and architecture to play a decisive role at in-plain grain boundaries, where they determine which molecular subunits are exposed at the interface, and hence govern the formation of either energy barriers or energy traps at the grain boundary. To retain efficient charge transport, energy barriers should be preferred as the charge migration across the boundary is then limited by barrier gap diffusion rather than by the escape time from energetic traps.

Merits of the Acceptor-Donor-Acceptor Architecture Based on the relationship between molecular architecture and orientation, we could rationalize the success of the acceptordonor-acceptor (ADA) architecture for molecular donor materials, used in the world-record system $\mathrm{C}_{60} / \mathrm{D} 5 \mathrm{M}$. The electrostatic layout of these ADA compounds leads to a destabilization of hole carriers in the absence of long-range ordering. In the case of long-range coherence in a thin-film setup, however, the face-on orientation of the D5M component corresponds to the lowest-ionization-energy configuration, then characterized by an only slightly (rather than strongly) negative electrostatic contribution. This face-on mode thus combines a sizeable photovoltaic gap and adequate interface polarity with strong optical absorption (due to the favourable alignment of the transition dipole moment) and fast out-of-plane transport (due to the favourable alignment of the $\pi$ - $\pi$-stacking direction) required for efficient charge separation and extraction. These advantages not only extend to $\mathrm{C}_{60} / \mathrm{D} 5 \mathrm{M}$, for which the face-on configuration has indeed been verified, but to ADA compounds in general. The ADA paradigm therefore appears a promising approach for the development of new donor materials. Similarly, the DAD pattern, if synthetically realizable, could be used for the synthesis of new non-fullerene acceptors with strong absorption profiles.

Calculation of Open-Circuit Voltages Across Planar Heterojunctions By computing the densities of states across planar donor-acceptor heterojunctions - with an account 
of both molecular orientation and thermal broadening - we calculated the charge-densitydependent open-circuit voltage from the quasi Fermi level splitting. The thus obtained voltages prove in excellent agreement with experimental voltages for typical steady-state charge densities at open-circuit conditions. The difference between the open-circuit voltage $V_{\text {oc }}$ and mean of the photovoltaic gap $\Gamma$ is largely effected by the thermal broadening $\sigma$ of the densities of states [199]. For crystalline organic semiconductors, $\sigma$ ranges between $40 \mathrm{meV}$ and $100 \mathrm{meV}$. As a result, we found the difference between $V_{\mathrm{oc}}$ and $\Gamma$ to range in between $0.7 \mathrm{eV}$ and $0.9 \mathrm{eV}$. This exceeds the voltage gap of around $0.5 \mathrm{eV}$ estimated from photoemission spectra [152]. It remains to be seen whether this discrepancy might be due to a systematic underestimation of the photovoltaic gap by photoemission techniques, where the onsets of the HOMO and LUMO peaks are used: The latter are not only subject to thermal broadening, but also, due to the measurement process, vibrational broadening and shifts. This way, for instance, energetic disorder strengths of $250 \mathrm{meV}$ have been extracted for pentacene - far larger than what is physically plausible for such a high-mobility system. Meanwhile, the $0.7-0.9 \mathrm{eV}$ are consistent with the $0.5-0.6 \mathrm{eV}$ difference between $\mathrm{CT}$ energies and $V_{\text {oc }}$ reported by optical measurements [196] when taking into account the CT binding energy. Indeed, our computed CT energies confirm this empirical relationship, yielding a slightly lower gap of 0.4-0.5 eV. The microscopic origin of this gap remained, however, impervious to our simulations, as explicit dynamics of charge-carrier and charge transfer state recombination would have to be addressed.

Pathway for Charge Splitting and Detrapping We have proposed a mechanism for electron-hole splitting at donor-acceptor interfaces that harvests mesoscale fields during the initial steps of charge separation, crucial in organic solar cells. To this end, we have shown how the in-built interface polarity results from the asymmetric stabilization (or destabilization) of electrons and holes on either side of the interface, as dictated by molecular architecture and orientation. Interface roughness then creates a field gradient that assists charge splitting and enables minority-carrier detrapping if the interface is poled correctly. Due to their mesoscopic origin, the charge splitting fields persist both in the case of fine intermixing and domain interpenetration. The resulting push-out mechanism can in principle be engineered such that the charge transfer (CT) state becomes isoenergetic with the photovoltaic gap - thus providing a route for barrier-less electron-hole separation. The molecular orientation and degree of interface roughness control the amount by which the CT-state energy is increased or decreased. The largest push-out forces can be realized in low hole- and electron-energy configurations of the donor and acceptor, respectively - at the cost of a decreased photovoltaic gap. We have rationalized that operation just above an isopolar point, characterized by flat electrostatic level profiles, is most desirable. Realization of such a scenario may however be challenging, in particular in systems where both the donor and acceptor component are strongly polar and can thus render charge generation ineffective if arrested in a negativepolarity orientation. Still, this electrostatic charge splitting pathway with its direct link to interface structuring could explain why some photovoltaic systems display a temperature- 
independent $[186,187,200]$ rather than the expected temperature-activated charge generation behaviour [201].

Vice and Virtue of Low-Energy Configurations We have attributed a key role to lowest-energy configurations at interfaces: These configurations not only appear to categorically determine the open-circuit voltage in organic solar cells, but also (see above) facilitate charge splitting and detrapping. Beyond low-energy orientations, low-energy packing modes are also more likely to promote energy barriers at grain boundaries, preferred over energy traps. In solar cells, however, they imply a reduced photovoltaic gap, which linearly enters both the CT-state energy and open-circuit voltage. In many cases the Frenkel exciton energy still leaves room for increasing the CT-state energy (and hence voltage) without stalling device functionality nor impeding charge push-out effects. An open question is therefore whether precise morphological tuning can indeed harvest what energetically resembles a passed-up opportunity. Even in bulk heterojunctions, the device characteristics continue to be pinned by these lowest-energy configurations - despite the "traditional" picture of bulk heterojunctions suggesting the coexistence of a variety of donor-acceptor interfaces with different orientations, in-plane extensions, degrees of intermixing, and hence different energetics [202]. Nevertheless, the open-circuit voltage of bulk heterojunctions is usually on the order of $0.1 \mathrm{eV}$ smaller than in the planar setup, and still correlated with a single CT energy extracted from absorption and electroluminescence. This energetic similarity of bulk and planar heterojunctions remains enigmatic; to identify its origin, more details would have to be known about the microscopic structure of these junctions. In particular, the "centro-symmetric" layout of bulk morphologies may have to be reassessed, as in-situ growth studies [107] and ellipsometric characterizations [62] indicate that donor-acceptor mixtures are not only well phase-separated, but in fact macroscopically ordered with respect to the substrate. In view of this residual preferential ordering, future computational studies will hopefully resolve to which degree mesoscopic interactions persist in both planar and bulk heterojunctions.

The derivation of quantitative structure-property relationships and design principles summarized above were made possible by the development of new computational methods targeting the morphological and electronic properties of organic semiconductors with a systematic link to the molecular structures. Most importantly, the presented long-range polarized embedding technique provided quantitative access to the energetics of charges and CT states. Built on a perturbative approach, the method is efficiently parametrized from first principles and applicable to large atomistic models incorporating thousands of molecules at an affordable computational expense. Beyond organic heterostructures and grain boundaries, it enabled, for example, the study of the formation of low-energy states and spatial energetic correlations in large models of polymeric mesophases incorporating long-range conformational disorder.

The method as applied here is, however, not yet suited to investigate the energetics of states with a strongly quantum-mechanical character, such as non-integer CT states. An extension of the scheme to incorporate a quantum-mechanical description of the embedded 
state is currently under development. On the quantum level, many-body Green's functions approaches are the method of choice due to their accuracy and computational efficiency: The GW-BSE formalism, for example, significantly improves on time-dependent density functional theory by accounting for a dynamically screened Coulomb interaction in the self-energy operator $(\mathrm{GW})$ and an excitonic electron-hole interaction described by the Bethe-Salpeter equation (BSE) [195].

Highly relevant for the modelling of devices, a further extension of the embedding approach should target the description of metal-organic interfaces, notably injection barriers: The effect of image charges on contact potentials can be modelled directly [203], whereas charge equilibration across the interface probably has to be addressed by coupling the particle-based description to a Poisson-Fermi solver [127].

An additional challenge lies in how accurate energy calculations can be integrated efficiently into explicit simulations of charge-carrier and exciton dynamics using kinetic MonteCarlo techniques. Encoding long-range interactions into the single-particle energy landscapes already implies a possibly inappropriate approximation to many-body polarization effects, since these result both in an effectively reduced attraction between charge carriers of the same polarity and reduced repulsion of charge carriers of opposite polarity not captured by the single-particle approximation. Within monodisperse systems, the associated charge concentration effects could be modelled using an effective distance-dependent dielectric function. However, whether a similar approach would hold for more complex polydisperse systems and heterostructures is uncertain. Yet more complicated is the incorporation of long-range electrostatics and polarization into systems with partially delocalized carriers, where explicit propagation of the wave functions via surface hopping techniques becomes necessary [204]. Such an extension is highly relevant as the degree to which polarization leads to an additional localization of charge carriers is still unresolved.

Meanwhile, morphology simulations continue to present one of the greatest challenges in the modelling of organic semiconductors due to the inherent large length and time scales that even in experiments occasionally result in kinetically arrested non-equilibrium structures [107]. Obtained using diverse approaches, the morphologies studied in this work retained their strictly model-like character, hence limiting the range of studies for which they are suited: Many relied on preassembled and experimentally determined input structures, others only provide a phenomenological description of complex morphological features such as grain boundaries or rough interfaces. Finally, those that do provide atomistic details are prone to finite-size effects that may impact the energy landscape of electronic states, here identified even for amorphous mesophases of dipolar molecules.

Predictive modelling of at least local structuring should, however, be possible. Crystalstructure prediction techniques could, for example, be applied to organic-organic interfaces to resolve molecular packing and orientation. For non-local structuring, particularly relevant in the complex organization of bulk heterojunctions, strictly predictive approaches remain elusive. The most promising simulation strategies thus combine bottom-up with top-down 
modelling protocols: The former provide the link to the molecular detail, whereas the latter capture supramolecular features such as lamellar ordering or domain formation. On the mesoscopic, top-down level, both field- [205] and particle-based approaches [72] coupled to an atomistic, bottom-up description are conceivable. The polymeric mesophases studied in this work [74] give an impression of what can be achieved with such approaches, hopefully providing a baseline for future method development. 


\section{ACKNOWLEDGEMENTS}

Diese Arbeit erstand auch aus der Wechselwirkung mit Ratgebern und Unterstützern, promovierenden und promovierten Mitstreitern, Theoretikern und Experimentatoren aus der Physik, Chemie, und den Materialwissenschaften - Mentoren, Kollegen und Freunden, denen ich an dieser Stelle herzlichen danken möchte:

Ich danke Herrn Professor Kurt Kremer für seine Unterstützung in fachlichen und administrativen Belangen, wie auch die Gelegenheit zum Brückenschlag zwischen dem MPIP in Mainz und meiner Alma Mater in Heidelberg.

Ich danke Dr. Denis Andrienko für sein breitgefächertes und ermutigendes Engagement. Dazu gehört unter anderem die praktische Lektion, die Segel nicht zu früh zu streichen.

Ich danke Herrn Professor Ulrich Schwarz für die kurzfristige Übernahme des Zweitgutachtens. Zudem bin ich ihm für ein Beratungsgespräch verbunden, das meine Entscheidung für die Theoriegruppe in Mainz bedingt und somit im Rückblick große Tragweite für mich entfaltet hat.

Ich danke Dr. Patrick Gemünden und P.D. Kostas Daoulas, ohne die polymere, mesoskopische Ambitionen wohl nur mikroskopische Resultate hervorgebracht hätten, für die Zusammenarbeit.

Ich danke derzeitigen und ehemaligen Mitstreitern aus der Organic-Electronics Group, insbesondere Dr. Björn Baumeier, Dr. Falk May, Pascal Kordt, Anton Melnyk und Jens Wehner, ohne die viele fachliche Diskussionen nicht so schlagfertig (und Code-Mergers nicht so spektakulär) ausgefallen wären.

Ich danke den Experimentatoren Dr. Chris Elschner, Dr. Max Tietze, Dr. Christian Körner, Dr. Johannes Widmer aus Dresden, sowie Dr. Selina Olthof und Dr. Dirk Hertel aus Köln, die geholfen haben, einem theoretisch-physikalischen Realitätsverlust entgegenzuwirken.

Ich danke meinen derzeitigen wie ehemaligen Bürokollegen Dr. Jarek Paturej, Dr. Claudia Mangold, Dr. Björn Baumeier, Dr. Joseph Rudzinski und Dr. Nancy Forero, die mit mir den Raum auch zu Zeiten gedanklicher Versenkung rücksichtsvoll geteilt haben.

Ich danke den Gruppenmitgliedern aus: Bolivien, Brasilien, China, Frankreich, Griechenland, Indien, dem Iran, Irland, Israel, Italien, Japan, Kolumbien, Russland, Schottland, Slovenien, Spanien, Südkorea, Taiwan, der Türkei, Ukraine und den USA dafür, ein Stück der weiten Welt in die Nähe meines kleinen Büros getragen zu haben.

Ich danke meinen Eltern für ihre Unterstützung und Anteilnahme, die auch durch unwillkürlich verklausulierte Erklärungen meiner Tätigkeit keinen Schaden nahm.

Ich danke Lili für Rückhalt, Zuversicht und Güte. 


\section{List of Publications}

Some of the sections and chapters of this thesis are adaptations and/or extensions of the following publications and manuscripts:

- C. Poelking and D. Andrienko: Design Rules for Organic Donor-Acceptor Heterojunctions: Pathway for Charge Splitting and Detrapping. Journal of the American Chemical Society 137(19) 6320-6326 (2015)

- P. Gemünden, C. Poelking, K. Kremer, K. Daoulas and D. Andrienko: Effect of Mesoscale Ordering on the Density of States in Polymeric Semiconductors. Macromolecular Rapid Communications 36(11) 1047-1053 (2015)

- C. Poelking, M. Tietze, C. Elschner, S. Olthof, D. Hertel, B. Baumeier, F. Würthner, K. Meerholz, K. Leo and D. Andrienko: Impact of Mesoscale Order on Open-Circuit Voltage in Organic Solar Cells. Nature Materials 14(4) 434-439 (2014)

- C. Poelking, K. Daoulas, A. Troisi and D. Andrienko: Morphology and Charge Transport in P3HT: A Theorist's Perspective. Advances in Polymer Science 265 139-180 (2014)

- C. Poelking and D. Andrienko: Long-Range Embedding of Molecular Ions and Excitations in a Polarizable Molecular Environment. Submitted.

- F. Steiner, C. Poelking, D. Niedzialek, D. Andrienko and J. Nelson: Influence of Orientation Mismatch on Charge Transport Across Grain Boundaries in Triisopropylsilylethynyl (TIPS) Pentacene Thin Films. In preparation.

- M. Schwarze, W. Tress, B. Beyer, F. Gao, R. Scholz, D. Kasemann and K. Leo in collaboration with C. Poelking and D. Andrienko: Bandstructure Engineering in Organic Semiconductors. In preparation.

Previous work by the author of this thesis in the field of organic electronics and photovoltaics is published in the following journal articles:

- C. Poelking and D. Andrienko: Effect of Paracrystallinity, Polymorphism and Regioregularity on Charge Transport in Poly(3-Hexyl-Thiophene) Nanofibers. Macromolecules 46 (22) 8941-8956 (2013) 
- P. Gemünden, C. Poelking, K. Kremer, D. Andrienko and K. Daoulas: Nematic Ordering, Conjugation and Density of States of Soluble Polymeric Semiconductors. Macromolecules 46 (14) 5762-5774 (2013)

- C. Poelking, E. Cho, A. Malafeev, V. Ivanov, K. Kremer, C. Risko, J.-L. Brédas and D. Andrienko: Characterization of Charge-Carrier Transport in Semicrystalline Polymers: Electronic Couplings, Site Energies, and Charge-Carrier Dynamics in Poly(bithiophenealt-thienothiophene) [PBTTT] Journal of Physical Chemistry C 117 (4) 1633-1640 (2013)

- B. Baumeier, O. Stenzel, C. Poelking, D. Andrienko and V. Schmidt: Stochastic Modeling of Molecular Charge-Transport Networks. Physical Review B 86184202 (2012)

- R. Lovrincic, J. Trollmann, C. Poelking, J. Brill, J. Schöneboom, C. Lennartz and A. Pucci: Orientation of Non-Planar Molecules in Polycrystalline Layers from Infrared Spectra: Corechlorinated Naphthalene Tetracarboxylic Diimides. Journal of Physical Chemistry C 116 (9) 5757-5763 (2012)

- A. Ojala, A. Petersen, A. Fuchs, R. Lovrincic, C. Poelking, J. Trollmann, A. Pucci, C. Lennartz, H. Reichelt, P. Erk and F. Würthner: Merocyanine/C60 Planar Heterojunction Solar Cells: Effect of Dye Orientation on Exciton Dissociation and Solar Cell Performance. Advanced Functional Materials 22(1) 86-96 (2011)

- A. Chatten, D. Farrell, R. Bose, A. Dixon, C. Poelking, K. Godel, M. Mazzer and K. Barnham: Luminescent and Geometric Concentrators for Building Integrated Photovoltaics. Proceedings of the 37th IEEE Photovoltaic Specialists Conference PVSC 852-857 (2011) 


\section{BIBLIOGRAPHY}

[1] V. Walatka, M. Labes, and Jerome Perlstein. Polysulfur Nitride - a One-Dimensional Chain with a Metallic Ground State. Phys. Rev. Lett., 31(18):1139-1142, 1973.

[2] C. Chiang, C. Fincher, Y. Park, A. Heeger, H. Shirakawa, E. Louis, S. Gau, and Alan MacDiarmid. Electrical Conductivity in Doped Polyacetylene. Phys. Rev. Lett., 39(17): 1098-1101, 1977.

[3] A. Tsumura, H. Koezuka, and T. Ando. Macromolecular electronic device: Field-effect transistor with a polythiophene thin film. Appl. Phys. Lett., 49(18):1210, 1986.

[4] C. W. Tang. Two-layer organic photovoltaic cell. Appl. Phys. Lett., 48(2):183, 1986.

[5] C. W. Tang and S. A. van Slyke. Organic electroluminescent diodes. Appl. Phys. Lett., 51(12):913, 1987.

[6] Jean Roncali, Philippe Leriche, and Philippe Blanchard. Molecular Materials for Organic Photovoltaics: Small is Beautiful. Adv. Mater., 26(23):3821-3838, 2014.

[7] Antonio Facchetti. $\pi$-Conjugated Polymers for Organic Electronics and Photovoltaic Cell Applications. Chem. Mater., 23(3):733-758, 2011.

[8] Steve Albrecht, Silvia Janietz, Wolfram Schindler, Johannes Frisch, Jona Kurpiers, Juliane Kniepert, Sahika Inal, Patrick Pingel, Konstantinos Fostiropoulos, Norbert Koch, and Dieter Neher. Fluorinated Copolymer PCPDTBT with Enhanced Open-Circuit Voltage and Reduced Recombination for Highly Efficient Polymer Solar Cells. J. Am. Chem. Soc., 134(36):14932-14944, 2012.

[9] Roland Fitzner, Elena Mena-Osteritz, Amaresh Mishra, Gisela Schulz, Egon Reinold, Matthias Weil, Christian Körner, Hannah Ziehlke, Chris Elschner, Karl Leo, Moritz Riede, Martin Pfeiffer, Christian Uhrich, and Peter Bäuerle. Correlation of $\pi$ Conjugated Oligomer Structure with Film Morphology and Organic Solar Cell Performance. J. Am. Chem. Soc., 134(27):11064-11067, 2012.

[10] Il Kang, Hui-Jun Yun, Dae Sung Chung, Soon-Ki Kwon, and Yun-Hi Kim. Record High Hole Mobility in Polymer Semiconductors via Side-Chain Engineering. J. Am. Chem. Soc., 135(40):14896-14899, 2013. 
[11] Youngkyoo Kim, Steffan Cook, Sachetan M. Tuladhar, Stelios A. Choulis, Jenny Nelson, James R. Durrant, Donal D. C. Bradley, Mark Giles, Iain McCulloch, Chang-Sik Ha, and Moonhor Ree. A strong regioregularity effect in self-organizing conjugated polymer films and high-efficiency polythiophene:fullerene solar cells. Nat. Mater., 5(3):197-203, 2006.

[12] Felix Peter Vinzenz Koch, Jonathan Rivnay, Sam Foster, Christian Müller, Jonathan M. Downing, Ester Buchaca-Domingo, Paul Westacott, Liyang Yu, Mingjian Yuan, Mohammed Baklar, Zhuping Fei, Christine Luscombe, Martyn A. McLachlan, Martin Heeney, Garry Rumbles, Carlos Silva, Alberto Salleo, Jenny Nelson, Paul Smith, and Natalie Stingelin. The impact of molecular weight on microstructure and charge transport in semicrystalline polymer semiconductors - poly(3-hexylthiophene), a model study. Progress in Polymer Science, 38(12):1978-1989, 2013.

[13] Ying Diao, Leo Shaw, Zhenan Bao, and Stefan C. B. Mannsfeld. Morphology control strategies for solution-processed organic semiconductor thin films. Energy Environ. Sci., $7(7): 2145-2159,2014$.

[14] Marc Baldo, Miriam Deutsch, Paul Burrows, Herman Gossenberger, Michael Gerstenberg, Vladimir Ban, and Stephen Forrest. Organic Vapor Phase Deposition. Adv. Mater., 10(18):1505-1514, 1998.

[15] Markus Schwoerer, Hans Christoph Wolf, and William D. Brewer. Organic molecular solids. Physics textbook. Wiley-VCH, Weinheim, 2007. ISBN 3527405402.

[16] Volkhard May and Oliver Kühn. Charge and Energy Transfer Dynamics in Molecular Systems. Wiley-VCH, 3rd, revised and enlarged edition edition, 2011. ISBN 3527407324.

[17] Alessandro Troisi. Charge transport in high mobility molecular semiconductors: classical models and new theories. Chem. Soc. Rev., 40(5):2347, 2011.

[18] Ilhan Yavuz, Blanton N. Martin, Jiyong Park, and K. N. Houk. Theoretical Study of the Molecular Ordering, Paracrystallinity, and Charge Mobilities of Oligomers in Different Crystalline Phases. J. Am. Chem. Soc., 137(8):2856-2866, 2015.

[19] S. V. Novikov, D. H. Dunlap, V. M. Kenkre, P. E. Parris, and A. V. Vannikov. Essential Role of Correlations in Governing Charge Transport in Disordered Organic Materials. Phys. Rev. Lett., 81(20):4472, 1998.

[20] W. F. Pasveer, J. Cottaar, C. Tanase, R. Coehoorn, P. A. Bobbert, P. W. M. Blom, D. M. de Leeuw, and M. A. J. Michels. Unified Description of Charge-Carrier Mobilities in Disordered Semiconducting Polymers. Phys. Rev. Lett., 94(20), 2005.

[21] M. Bouhassoune, S.L.M. van Mensfoort, P.A. Bobbert, and R. Coehoorn. Carrierdensity and field-dependent charge-carrier mobility in organic semiconductors with correlated Gaussian disorder. Organic Electronics, 10(3):437-445, 2009. 
[22] Oleksandr V. Mikhnenko, Martijn Kuik, Jason Lin, Niels van der Kaap, Thuc-Quyen Nguyen, and Paul W. M. Blom. Trap-Limited Exciton Diffusion in Organic Semiconductors. Adv. Mater., 26(12):1912-1917, 2014.

[23] Benjamin C-K. Tee, Chao Wang, Ranulfo Allen, and Zhenan Bao. An electrically and mechanically self-healing composite with pressure- and flexion-sensitive properties for electronic skin applications. Nat. Nanotechnol., 7(12):825-832, 2012.

[24] Jiri Janata and Mira Josowicz. Conducting polymers in electronic chemical sensors. Nat. Mater., 2(1):19-24, 2003.

[25] Claire M. Lochner, Yasser Khan, Adrien Pierre, and Ana C. Arias. All-organic optoelectronic sensor for pulse oximetry. Nature Communications, 5:5745, 2014.

[26] Rico Meerheim, Christian Körner, and Karl Leo. Highly efficient organic multi-junction solar cells with a thiophene-based donor material. Appl. Phys. Lett., 105(6):063306, 2014 .

[27] Press Release: Heliatek consolidates its technology leadership by establishing a new world record for organic solar technology with a cell efficiency of $12 \%, 2013$.

[28] Pascal Kordt, Jeroen J. M. van der Holst, Mustapha Al Helwi, Wolfgang Kowalsky, Falk May, Alexander Badinski, Christian Lennartz, and Denis Andrienko. Modeling of Organic Light Emitting Diodes: From Molecular to Device Properties. Adv. Funct. Mater., 25(13):1955-1971, 2015.

[29] Deepak Venkateshvaran, Mark Nikolka, Aditya Sadhanala, Vincent Lemaur, Mateusz Zelazny, Michal Kepa, Michael Hurhangee, Auke Jisk Kronemeijer, Vincenzo Pecunia, Iyad Nasrallah, Igor Romanov, Katharina Broch, Iain McCulloch, David Emin, Yoann Olivier, Jerome Cornil, David Beljonne, and Henning Sirringhaus. Approaching disorder-free transport in high-mobility conjugated polymers. Nature, 515(7527): 384-388, 2014.

[30] Pierre-Luc T. Boudreault, Ahmed Najari, and Mario Leclerc. Processable Low-Bandgap Polymers for Photovoltaic Applications. Chem. Mater., 23(3):456-469, 2010.

[31] Michael Sommer, Sven Hüttner, and Mukundan Thelakkat. Donor-acceptor block copolymers for photovoltaic applications. J. Mater. Chem., 20(48):10788, 2010.

[32] Amaresh Mishra and Peter Bäuerle. Small Molecule Organic Semiconductors on the Move: Promises for Future Solar Energy Technology. Angew. Chem. Int. Edit., 51(9): 2020-2067, 2012.

[33] Priya J. Jadhav, Aseema Mohanty, Jason Sussman, Jiye Lee, and Marc A. Baldo. Singlet Exciton Fission in Nanostructured Organic Solar Cells. Nano Letters, 11(4):1495-1498, 2011. 
[34] D. N. Congreve, J. Lee, N. J. Thompson, E. Hontz, S. R. Yost, P. D. Reusswig, M. E. Bahlke, S. Reineke, T. Van Voorhis, and M. A. Baldo. External Quantum Efficiency Above $100 \%$ in a Singlet-Exciton-Fission-Based Organic Photovoltaic Cell. Science, 340 (6130):334-337, 2013.

[35] Xike Gao and Yunbin Hu. Development of n-type organic semiconductors for thin film transistors: a viewpoint of molecular design. Journal of Materials Chemistry C, 2(17): 3099, 2014.

[36] Yifan Zhang, Jaesang Lee, and Stephen R. Forrest. Tenfold increase in the lifetime of blue phosphorescent organic light-emitting diodes. Nature Communications, 5:5008, 2014.

[37] Shuzo Hirata, Yumi Sakai, Kensuke Masui, Hiroyuki Tanaka, Sae Youn Lee, Hiroko Nomura, Nozomi Nakamura, Mao Yasumatsu, Hajime Nakanotani, Qisheng Zhang, Katsuyuki Shizu, Hiroshi Miyazaki, and Chihaya Adachi. Highly efficient blue electroluminescence based on thermally activated delayed fluorescence. Nat. Mater., 14(3): 330-336, 2014.

[38] Seok-In Na, Seok-Soon Kim, Jang Jo, and Dong-Yu Kim. Efficient and Flexible ITOFree Organic Solar Cells Using Highly Conductive Polymer Anodes. Adv. Mater., 20 (21):4061-4067, 2008.

[39] Kjell Cnops, Barry P. Rand, David Cheyns, Bregt Verreet, Max A. Empl, and Paul Heremans. 8.4\% efficient fullerene-free organic solar cells exploiting long-range exciton energy transfer. Nature Communications, 5, 2014.

[40] Antonio Facchetti. Organic semiconductors: Made to order. Nat. Mater., 12(7):598-600, 2013.

[41] Neil D. Treat, Jennifer A. Nekuda Malik, Obadiah Reid, Liyang Yu, Christopher G. Shuttle, Garry Rumbles, Craig J. Hawker, Michael L. Chabinyc, Paul Smith, and Natalie Stingelin. Microstructure formation in molecular and polymer semiconductors assisted by nucleation agents. Nat. Mater., 12(7):628-633, 2013.

[42] Bong-Gi Kim, Eun Jeong Jeong, Jong Won Chung, Sungbaek Seo, Bonwon Koo, and Jinsang Kim. A molecular design principle of lyotropic liquid-crystalline conjugated polymers with directed alignment capability for plastic electronics. Nat. Mater., 12(7): 659-664, 2013.

[43] Ying Diao, Benjamin C-K. Tee, Gaurav Giri, Jie Xu, Do Hwan Kim, Hector A. Becerril, Randall M. Stoltenberg, Tae Hoon Lee, Gi Xue, Stefan C. B. Mannsfeld, and Zhenan Bao. Solution coating of large-area organic semiconductor thin films with aligned singlecrystalline domains. Nat. Mater., 12(7):665-671, 2013. 
[44] H. Bässler. Charge Transport in Disordered Organic Photoconductors: a Monte Carlo Simulation Study. physica status solidi (b), 175(1):15-56, 1993.

[45] Murat Mesta, Marco Carvelli, Rein J. de Vries, Harm van Eersel, Jeroen J. M. van der Holst, Matthias Schober, Mauro Furno, Björn Lüssem, Karl Leo, Peter Loebl, Reinder Coehoorn, and Peter A. Bobbert. Molecular-scale simulation of electroluminescence in a multilayer white organic light-emitting diode. Nat. Mater., 12(7):652-658, 2013.

[46] Reinhard Scholz, Regina Luschtinetz, Gotthard Seifert, Till Jägeler-Hoheisel, Christian Körner, Karl Leo, and Mathias Rapacioli. Review: Quantifying charge transfer energies at donor-acceptor interfaces in small-molecule solar cells with constrained DFTB and spectroscopic methods. J. Phys-Condens. Mat., 25(47):473201, 2013.

[47] Alessandro Troisi, David L. Cheung, and Denis Andrienko. Charge Transport in Semiconductors with Multiscale Conformational Dynamics. Phys. Rev. Lett., 102(11):116602, 2009 .

[48] Thorsten Vehoff, Yeon Sook Chung, Karen Johnston, Alessandro Troisi, Do Y. Yoon, and Denis Andrienko. Charge Transport in Self-Assembled Semiconducting Organic Layers: Role of Dynamic and Static Disorder. J. Phys. Chem. C, 114(23):10592-10597, 2010.

[49] David P McMahon and Alessandro Troisi. Organic semiconductors: impact of disorder at different timescales. ChemPhysChem, 11(10):2067-2074, 2010.

[50] Victor Rühle, Alexander Lukyanov, Falk May, Manuel Schrader, Thorsten Vehoff, James Kirkpatrick, Björn Baumeier, and Denis Andrienko. Microscopic Simulations of Charge Transport in Disordered Organic Semiconductors. J. Chem. Theory. Comput., 7(10):3335-3345, 2011.

[51] Richard M. Martin. Electronic structure: basic theory and practical methods. Cambridge University Press, Cambridge, UK ; New York, 2004. ISBN 0521782856.

[52] Attila Szabo and Neil S. Ostlund. Modern quantum chemistry: introduction to advanced electronic structure theory. Dover Publications, Mineola, N.Y, 1996. ISBN 0486691861.

[53] R. Ditchfield. Self-Consistent Molecular-Orbital Methods. IX. An Extended GaussianType Basis for Molecular-Orbital Studies of Organic Molecules. J. Chem. Phys., 54(2): $724,1971$.

[54] Daan Frenkel. Understanding molecular simulation: from algorithms to applications. Computational Science Series. Academic Press, San Diego, 2nd ed edition, 2002. ISBN 0122673514 . 
[55] H. J. C. Berendsen, J. P. M. Postma, W. F. van Gunsteren, A. DiNola, and J. R. Haak. Molecular dynamics with coupling to an external bath. J. Chem. Phys., 81(8):3684, 1984.

[56] Giovanni Bussi, Davide Donadio, and Michele Parrinello. Canonical sampling through velocity rescaling. J. Chem. Phys., 126(1):014101, 2007.

[57] H.J.C. Berendsen, D. van der Spoel, and R. van Drunen. GROMACS: A messagepassing parallel molecular dynamics implementation. Computer Physics Communications, 91(1-3):43-56, 1995.

[58] William L. Jorgensen and Julian. Tirado-Rives. The OPLS [optimized potentials for liquid simulations] potential functions for proteins, energy minimizations for crystals of cyclic peptides and crambin. J. Am. Chem. Soc., 110(6):1657-1666, 1988.

[59] David J. Wales and Jonathan P. K. Doye. Global Optimization by Basin-Hopping and the Lowest Energy Structures of Lennard-Jones Clusters Containing up to 110 Atoms. J. Phys. Chem. A, 101(28):5111-5116, 1997.

[60] Carl Poelking and Denis Andrienko. Effect of Polymorphism, Regioregularity and Paracrystallinity on Charge Transport in Poly(3-hexylthiophene) [P3ht] Nanofibers. Macromolecules, 46(22):8941-8956, 2013.

[61] Carl Poelking. Charge Transport Simulations in Polymeric Semiconductors. MSc Thesis, University of Heidelberg, 2013.

[62] D. Wynands, M. Erber, R. Rentenberger, M. Levichkova, K. Walzer, K.-J. Eichhorn, and M. Stamm. Spectroscopic ellipsometry characterization of vacuum-deposited organic films for the application in organic solar cells. Organic Electronics, 13(5):885-893, 2012.

[63] Kurt Kremer and Florian Müller-Plathe. Multiscale simulation in polymer science. Molecular Simulation, 28(8-9):729-750, 2002.

[64] Alexander P. Lyubartsev and Aatto Laaksonen. Calculation of effective interaction potentials from radial distribution functions: A reverse Monte Carlo approach. Phys. Rev. E, 52(4):3730-3737, 1995.

[65] Sergei Izvekov and Gregory A. Voth. A Multiscale Coarse-Graining Method for Biomolecular Systems. J. Phys. Chem. B, 109(7):2469-2473, 2005.

[66] M. Scott Shell. The relative entropy is fundamental to multiscale and inverse thermodynamic problems. J. Chem. Phys., 129(14):144108, 2008.

[67] David M. Huang, Roland Faller, Khanh Do, and Adam J. Moulé. Coarse-Grained Computer Simulations of Polymer/Fullerene Bulk Heterojunctions for Organic Photovoltaic Applications. J. Chem. Theory. Comput., 6(2):526-537, 2010. 
[68] Eric Jankowski, Hilary S. Marsh, and Arthi Jayaraman. Computationally Linking Molecular Features of Conjugated Polymers and Fullerene Derivatives to Bulk Heterojunction Morphology. Macromolecules, 46(14):5775-5785, 2013.

[69] Kyra N. Schwarz, Tak W. Kee, and David M. Huang. Coarse-grained simulations of the solution-phase self-assembly of poly(3-hexylthiophene) nanostructures. Nanoscale, $5(5): 2017,2013$.

[70] Alexander Lukyanov, Alexander Malafeev, Viktor Ivanov, Hsin-Lung Chen, Kurt Kremer, and Denis Andrienko. Solvated poly-(phenylene vinylene) derivatives: conformational structure and aggregation behavior. J. Mater. Chem., 20(46):10475, 2010.

[71] Victor Rühle, James Kirkpatrick, and Denis Andrienko. A multiscale description of charge transport in conjugated oligomers. J. Chem. Phys., 132(13):134103-9, 2010.

[72] Patrick Gemünden, Carl Poelking, Kurt Kremer, Denis Andrienko, and Kostas Ch. Daoulas. Nematic Ordering, Conjugation, and Density of States of Soluble Polymeric Semiconductors. Macromolecules, 46(14):5762-5774, 2013.

[73] Patrick Gemünden and Kostas Ch. Daoulas. Fluctuation spectra in polymer nematics and Frank elastic constants: a coarse-grained modelling study. Soft Matter, 11(3): $532-544,2015$.

[74] Patrick Gemünden, Carl Poelking, Kurt Kremer, Kostas Daoulas, and Denis Andrienko. Effect of Mesoscale Ordering on the Density of States of Polymeric Semiconductors. Macromolecular Rapid Communications, 36(11):1047-1053, 2015.

[75] Jean-Luc Brédas, David Beljonne, Veaceslav Coropceanu, and Jerome Cornil. ChargeTransfer and Energy-Transfer Processes in $\pi$-Conjugated Oligomers and Polymers: A Molecular Picture. Chem. Rev., 104(11):4971-5004, 2004.

[76] R. A. Marcus. On the Theory of Oxidation-Reduction Reactions Involving Electron Transfer. I. J. Chem. Phys., 24(5):966, 1956.

[77] Falk May. Charge-Transport Simulations in Organic Semiconductors. PhD thesis, Johannes-Gutenberg-Universität Mainz, 2012.

[78] Joshua Jortner. Temperature-dependent activation energy for electron transfer between biological molecules. J. Chem. Phys., 64(12):4860, 1976.

[79] Kamal Asadi, Auke J. Kronemeijer, Tobias Cramer, L. Jan Anton Koster, Paul W. M. Blom, and Dago M. de Leeuw. Polaron hopping mediated by nuclear tunnelling in semiconducting polymers at high carrier density. Nature Communications, 4:1710, 2013.

[80] Hermann Grabert and Ulrich Weiss. Quantum Tunneling Rates for Asymmetric DoubleWell Systems with Ohmic Dissipation. Phys. Rev. Lett., 54(15):1605-1608, 1985. 
[81] Matthew P. A. Fisher and Alan T. Dorsey. Dissipative Quantum Tunneling in a Biased Double-Well System at Finite Temperatures. Phys. Rev. Lett., 54(15):1609-1612, 1985.

[82] Nenad Vukmirovic and Lin-Wang Wang. Density of States and Wave Function Localization in Disordered Conjugated Polymers: A Large Scale Computational Study. J. Phys. Chem. B, 115(8):1792-1797, 2011.

[83] Tao Liu and Alessandro Troisi. Understanding the Microscopic Origin of the Very High Charge Mobility in PBTTT: Tolerance of Thermal Disorder. Adv. Funct. Mater., 24 (7):925-933, 2014.

[84] D. Porezag, Th. Frauenheim, Th. Köhler, G. Seifert, and R. Kaschner. Construction of tight-binding-like potentials on the basis of density-functional theory: Application to carbon. Phys. Rev. B, 51(19):12947-12957, 1995.

[85] M. Elstner, D. Porezag, G. Jungnickel, J. Elsner, M. Haugk, T. Aernouts, S. Suhai, and G. Seifert. Self-consistent-charge density-functional tight-binding method for simulations of complex materials properties. Phys. Rev. B, 58(11):7260-7268, 1998.

[86] Haibo Ma, Ting Qin, and Alessandro Troisi. Electronic Excited States in Amorphous MEH-PPV Polymers from Large-Scale First Principles Calculations. J. Chem. Theory. Comput., 10(3):1272-1282, 2014.

[87] Troy Van Voorhis, Tim Kowalczyk, Benjamin Kaduk, Lee-Ping Wang, Chiao-Lun Cheng, and Qin Wu. The Diabatic Picture of Electron Transfer, Reaction Barriers, and Molecular Dynamics. Annual Review of Physical Chemistry, 61(1):149-170, 2010.

[88] Qin $\mathrm{Wu}$ and Troy Van Voorhis. Extracting electron transfer coupling elements from constrained density functional theory. J. Chem. Phys., 125(16):164105, 2006.

[89] Björn Baumeier, James Kirkpatrick, and Denis Andrienko. Density-functional based determination of intermolecular charge transfer properties for large-scale morphologies. Phys. Chem. Chem. Phys., 12(36):11103, 2010.

[90] James Kirkpatrick. An approximate method for calculating transfer integrals based on the ZINDO Hamiltonian. Int. J. Quantum Chem., 108(1):51-56, 2008.

[91] Veaceslav Coropceanu, Jérôme Cornil, Demetrio A. da Silva Filho, Yoann Olivier, Robert Silbey, and Jean-Luc Brédas. Charge Transport in Organic Semiconductors. Chem. Rev., 107(4):926-952, 2007.

[92] Daniel T Gillespie. A general method for numerically simulating the stochastic time evolution of coupled chemical reactions. J. Comput. Phys., 22(4):403-434, 1976.

[93] Kristen A. Fichthorn and W. H. Weinberg. Theoretical foundations of dynamical Monte Carlo simulations. J. Chem. Phys., 95(2):1090, 1991. 
[94] Alexander Lukyanov and Denis Andrienko. Extracting nondispersive charge carrier mobilities of organic semiconductors from simulations of small systems. Phys. Rev. B, 82(19):193202, 2010.

[95] Björn Baumeier, Ole Stenzel, Carl Poelking, Denis Andrienko, and Volker Schmidt. Stochastic modeling of molecular charge transport networks. Phys. Rev. B, 86(18), 2012 .

[96] S. Stodtmann, R. M. Lee, C. K. F. Weiler, and A. Badinski. Numerical simulation of organic semiconductor devices with high carrier densities. J. Appl. Phys., 112(11): 114909, 2012.

[97] Shane R. Yost and Troy Van Voorhis. Electrostatic Effects at Organic Semiconductor Interfaces: A Mechanism for "Cold" Exciton Breakup. J. Phys. Chem. C, 117(11): 5617-5625, 2013.

[98] Sean M. Ryno, Chad Risko, and Jean-Luc Brédas. Impact of Molecular Packing on Electronic Polarization in Organic Crystals: The Case of Pentacene vs TIPS-Pentacene. J. Am. Chem. Soc., 136(17):6421-6427, 2014.

[99] Julien Idé, Raphaël Méreau, Laurent Ducasse, Frédéric Castet, Harald Bock, Yoann Olivier, Jérôme Cornil, David Beljonne, Gabriele D'Avino, Otello Maria Roscioni, Luca Muccioli, and Claudio Zannoni. Charge Dissociation at Interfaces between Discotic Liquid Crystals: The Surprising Role of Column Mismatch. J. Am. Chem. Soc., 136 (7):2911-2920, 2014.

[100] Falk May, Björn Baumeier, Christian Lennartz, and Denis Andrienko. Can Lattice Models Predict the Density of States of Amorphous Organic Semiconductors? Phys. Rev. Lett., 109(13), 2012.

[101] Anthony J. Stone. Distributed Multipole Analysis: Stability for Large Basis Sets. J. Chem. Theory. Comput., 1(6):1128-1132, 2005.

[102] Gabriele D’Avino, Luca Muccioli, Claudio Zannoni, David Beljonne, and Zoltán G. Soos. Electronic Polarization in Organic Crystals: A Comparative Study of Induced Dipoles and Intramolecular Charge Redistribution Schemes. J. Chem. Theory. Comput., 10(11):4959-4971, 2014.

[103] B.T. Thole. Molecular polarizabilities calculated with a modified dipole interaction. Chem. Phys., 59(3):341-350, 1981.

[104] Steffen Duhm, Georg Heimel, Ingo Salzmann, Hendrik Glowatzki, Robert L. Johnson, Antje Vollmer, Jürgen P. Rabe, and Norbert Koch. Orientation-dependent ionization energies and interface dipoles in ordered molecular assemblies. Nat. Mater., 7(4):326$332,2008$. 
[105] Georg Heimel, Ingo Salzmann, Steffen Duhm, and Norbert Koch. Design of Organic Semiconductors from Molecular Electrostatics. Chem. Mater., 23(3):359-377, 2011.

[106] Carl Poelking, Max Tietze, Chris Elschner, Selina Olthof, Dirk Hertel, Björn Baumeier, Frank Würthner, Klaus Meerholz, Karl Leo, and Denis Andrienko. Impact of mesoscale order on open-circuit voltage in organic solar cells. Nat. Mater., 14(4):434-439, 2014.

[107] R. Banerjee, J. Novák, C. Frank, C. Lorch, A. Hinderhofer, A. Gerlach, and F. Schreiber. Evidence for Kinetically Limited Thickness-Dependent Phase Separation in Organic Thin-Film Blends. Phys. Rev. Lett., 110(18), 2013.

[108] A.J. Stone and M. Alderton. Distributed multipole analysis. Molecular Physics, 56(5): 1047-1064, 1985.

[109] A.J. Stone. Distributed polarizabilities. Molecular Physics, 56(5):1065-1082, 1985.

[110] A. J Stone. The Theory of intermolecular forces. Clarendon Press, Oxford, 1997. ISBN $019855883 X$ 9780198558835.

[111] Christof Hättig. Recurrence relations for the direct calculation of spherical multipole interaction tensors and Coulomb-type interaction energies. Chem. Phys. Lett., 260(3-4): 341-351, 1996.

[112] U. Chandra Singh and Peter A. Kollman. An approach to computing electrostatic charges for molecules. J. Comput. Chem., 5(2):129-145, 1984.

[113] Lisa Emily Chirlian and Michelle Miller Francl. Atomic charges derived from electrostatic potentials: A detailed study. J. Comput. Chem., 8(6):894-905, 1987.

[114] Curt M. Breneman and Kenneth B. Wiberg. Determining atom-centered monopoles from molecular electrostatic potentials. The need for high sampling density in formamide conformational analysis. J. Comput. Chem., 11(3):361-373, 1990.

[115] Piet Th. van Duijnen and Marcel Swart. Molecular and Atomic Polarizabilities: Thole's Model Revisited. J. Phys. Chem. A, 102(14):2399-2407, 1998.

[116] Jon Applequist, James R. Carl, and Kwok-Kueng Fung. Atom dipole interaction model for molecular polarizability. Application to polyatomic molecules and determination of atom polarizabilities. J. Am. Chem. Soc., 94(9):2952-2960, 1972.

[117] Pengyu Ren and Jay W. Ponder. Polarizable Atomic Multipole Water Model for Molecular Mechanics Simulation. J. Phys. Chem. B, 107(24):5933-5947, 2003.

[118] Christof Hättig and Bernd Artur Heß. Calculation of orientation-dependent doubletensor moments for Coulomb-type intermolecular interactions. Molecular Physics, 81 (4):813-824, 1994. 
[119] P. P. Ewald. Die Berechnung optischer und elektrostatischer Gitterpotentiale. Annalen der Physik, 369(3):253-287, 1921.

[120] M. Leslie. DL_multi - A molecular dynamics program to use distributed multipole electrostatic models to simulate the dynamics of organic crystals. Molecular Physics, 106(12):1567-1578, 2008.

[121] W. Smith. Point Multipoles in the Ewald Summation (Revisited). CCP5 Newsletter, 46, 1998.

[122] E. R. Smith. Electrostatic Energy in Ionic Crystals. Proceedings of the Royal Society of London. A. Mathematical and Physical Sciences, 375(1763):475-505, 1981.

[123] Paul S. Crozier, Richard L. Rowley, Eckhard Spohr, and Douglas Henderson. Comparison of charged sheets and corrected 3d Ewald calculations of long-range forces in slab geometry electrolyte systems with solvent molecules. J. Chem. Phys., 112(21):9253, 2000 .

[124] Simon W. De Leeuw and John W. Perram. Electrostatic lattice sums for semi-infinite lattices. Molecular Physics, 37(4):1313-1322, 1979.

[125] John G. Kirkwood. Theory of Solutions of Molecules Containing Widely Separated Charges with Special Application to Zwitterions. J. Chem. Phys., 2(7):351, 1934.

[126] Kyohei Takae and Akira Onuki. Applying electric field to charged and polar particles between metallic plates: Extension of the Ewald method. J. Chem. Phys., 139(12): 124108, 2013.

[127] Martin Oehzelt, Norbert Koch, and Georg Heimel. Organic semiconductor density of states controls the energy level alignment at electrode interfaces. Nature Communications, 5, 2014.

[128] Hannah Bürckstümmer, Elena V. Tulyakova, Manuela Deppisch, Martin R. Lenze, Nils M. Kronenberg, Marcel Gsänger, Matthias Stolte, Klaus Meerholz, and Frank Würthner. Efficient Solution-Processed Bulk Heterojunction Solar Cells by Antiparallel Supramolecular Arrangement of Dipolar Donor-Acceptor Dyes. Angew. Chem. Int. Edit., 50(49):11628-11632, 2011.

[129] Jean-Luc Brédas, Joseph E. Norton, Jérôme Cornil, and Veaceslav Coropceanu. Molecular Understanding of Organic Solar Cells: The Challenges. Accounts Chem. Res., 42 (11):1691-1699, 2009.

[130] Tracey M. Clarke and James R. Durrant. Charge Photogeneration in Organic Solar Cells. Chem. Rev., 110(11):6736-6767, 2010. 
[131] Letian Dou, Jingbi You, Ziruo Hong, Zheng Xu, Gang Li, Robert A. Street, and Yang Yang. 25th Anniversary Article: A Decade of Organic/Polymeric Photovoltaic Research. Adv. Mater., 25(46):6642-6671, 2013.

[132] A. Wilke, P. Amsalem, J. Frisch, B. Bröker, A. Vollmer, and N. Koch. Electric fields induced by energy level pinning at organic heterojunctions. Appl. Phys. Lett., 98(12): 123304, 2011.

[133] David Beljonne, Jérôme Cornil, Luca Muccioli, Claudio Zannoni, Jean-Luc Brédas, and Frédéric Castet. Electronic Processes at Organic-Organic Interfaces: Insight from Modeling and Implications for Opto-electronic Devices. Chem. Mater., 23(3):591-609, 2011.

[134] David P. McMahon, David L. Cheung, and Alessandro Troisi. Why Holes and Electrons Separate So Well in Polymer/Fullerene Photovoltaic Cells. J. Phys. Chem. Lett., 2(21): 2737-2741, 2011.

[135] A. Liu, S. Zhao, S.-B. Rim, J. Wu, M. Könemann, P. Erk, and P. Peumans. Control of Electric Field Strength and Orientation at the Donor-Acceptor Interface in Organic Solar Cells. Adv. Mater., 20(5):1065-1070, 2008.

[136] Brian A. Gregg. Entropy of Charge Separation in Organic Photovoltaic Cells: The Benefit of Higher Dimensionality. J. Phys. Chem. Lett., 2(24):3013-3015, 2011.

[137] Askat E. Jailaubekov, Adam P. Willard, John R. Tritsch, Wai-Lun Chan, Na Sai, Raluca Gearba, Loren G. Kaake, Kenrick J. Williams, Kevin Leung, Peter J. Rossky, and X.-Y. Zhu. Hot charge-transfer excitons set the time limit for charge separation at donor/acceptor interfaces in organic photovoltaics. Nat. Mater., 12(1):66-73, 2013.

[138] Domenico Caruso and Alessandro Troisi. Long-range exciton dissociation in organic solar cells. Proceedings of the National Academy of Sciences, 109(34):13498-13502, 2012 .

[139] Yongsheng Liu, Chun-Chao Chen, Ziruo Hong, Jing Gao, Yang (Michael) Yang, Huanping Zhou, Letian Dou, Gang Li, and Yang Yang. Solution-processed small-molecule solar cells: breaking the 10\% power conversion efficiency. Scientific Reports, 3, 2013.

[140] Shane R. Yost, Lee-Ping Wang, and Troy Van Voorhis. Molecular Insight Into the Energy Levels at the Organic Donor/Acceptor Interface: A Quantum Mechanics/Molecular Mechanics Study. J. Phys. Chem. C, 115(29):14431-14436, 2011.

[141] Yao-Tsung Fu, Chad Risko, and Jean-Luc Brédas. Intermixing at the PentaceneFullerene Bilayer Interface: A Molecular Dynamics Study. Adv. Mater., 25(6):878-882, 2013. 
[142] Luca Muccioli, Gabriele D’Avino, Roberto Berardi, Silvia Orlandi, Antonio Pizzirusso, Matteo Ricci, Otello Maria Roscioni, and Claudio Zannoni. Supramolecular Organization of Functional Organic Materials in the Bulk and at Organic/Organic Interfaces: A Modeling and Computer Simulation Approach. Topics in Current Chemistry, pages 1-63. Springer Berlin Heidelberg, 2013.

[143] Sébastien Mothy, Maxime Guillaume, Julien Idé, Frédéric Castet, Laurent Ducasse, Jérôme Cornil, and David Beljonne. Tuning the Interfacial Electronic Structure at Organic Heterojunctions by Chemical Design. J. Phys. Chem. Lett., 3(17):2374-2378, 2012 .

[144] Stijn Verlaak, David Beljonne, David Cheyns, Cedric Rolin, Mathieu Linares, Frédéric Castet, Jérôme Cornil, and Paul Heremans. Electronic Structure and Geminate Pair Energetics at Organic-Organic Interfaces: The Case of Pentacene/C60 Heterojunctions. Adv. Funct. Mater., 19(23):3809-3814, 2009.

[145] Mathieu Linares, David Beljonne, Jérôme Cornil, Kelly Lancaster, Jean-Luc Brédas, Stijn Verlaak, Alexander Mityashin, Paul Heremans, Andreas Fuchs, Christian Lennartz, Julien Idé, Raphaël Méreau, Philippe Aurel, Laurent Ducasse, and Frédéric Castet. On the Interface Dipole at the Pentacene-Fullerene Heterojunction: A Theoretical Study. J. Phys. Chem. C, 114(7):3215-3224, 2010.

[146] Julien Idé, Sébastien Mothy, Adrien Savoyant, Alain Fritsch, Philippe Aurel, Raphaël Méreau, Laurent Ducasse, Jérôme Cornil, David Beljonne, and Frédéric Castet. Interfacial dipole and band bending in model pentacene/C60 heterojunctions. Int. J. Quantum Chem., 113(4):580-584, 2013.

[147] S. W. de Leeuw, J. W. Perram, and E. R. Smith. Simulation of Electrostatic Systems in Periodic Boundary Conditions. I. Lattice Sums and Dielectric Constants. Proceedings of the Royal Society of London. A. Mathematical and Physical Sciences, 373(1752):27-56, 1980.

[148] Wei Chen, Dong-Chen Qi, Han Huang, Xingyu Gao, and Andrew T. S. Wee. Organic-Organic Heterojunction Interfaces: Effect of Molecular Orientation. Adv. Funct. Mater., 21(3):410-424, 2011.

[149] Hisao Ishii, Kiyoshi Sugiyama, Eisuke Ito, and Kazuhiko Seki. Energy Level Alignment and Interfacial Electronic Structures at Organic/Metal and Organic/Organic Interfaces. Adv. Mater., 11(8):605-625, 1999.

[150] I. G. Hill and A. Kahn. Organic semiconductor heterointerfaces containing bathocuproine. J. Appl. Phys., 86(8):4515, 1999. 
[151] F.J. Zhang, A. Vollmer, J. Zhang, Z. Xu, J.P. Rabe, and N. Koch. Energy level alignment and morphology of interfaces between molecular and polymeric organic semiconductors. Organic Electronics, 8(5):606-614, 2007.

[152] A. Wilke, J. Endres, U. Hörmann, J. Niederhausen, R. Schlesinger, J. Frisch, P. Amsalem, J. Wagner, M. Gruber, A. Opitz, A. Vollmer, W. Brütting, A. Kahn, and N. Koch. Correlation between interface energetics and open circuit voltage in organic photovoltaic cells. Appl. Phys. Lett., 101(23):233301, 2012.

[153] Georg Heimel and Norbert Koch. Molecular Orientation Dependence of the Ionization Energy of Pentacene in Thin Films. In Katharina Al-Shamery, Giles Horowitz, Helmut Sitter, and Horst-Günter Rubahn, editors, Interface Controlled Organic Thin Films, number 129 in Springer Proceedings in Physics, pages 141-145. Springer Berlin Heidelberg, 2009. ISBN 978-3-540-95929-8.

[154] Max L. Tietze, Wolfgang Tress, Steffen Pfützner, Christoph Schünemann, Lorenzo Burtone, Moritz Riede, Karl Leo, Koen Vandewal, Selina Olthof, Philip Schulz, and Antoine Kahn. Correlation of open-circuit voltage and energy levels in zinc-phthalocyanine: C60 bulk heterojunction solar cells with varied mixing ratio. Phys. Rev. B, 88(8):085119, 2013.

[155] Yoshiki Kinoshita, Taku Hasobe, and Hideyuki Murata. Controlling Open-Circuit Voltage of Organic Photovoltaic Cells by Inserting Thin Layer of Zn-Phthalocyanine at Pentacene/C 60 Interface. Jpn. J. Appl. Phys., 47(2):1234-1237, 2008.

[156] Manuel Schrader, Roland Fitzner, Moritz Hein, Chris Elschner, Björn Baumeier, Karl Leo, Moritz Riede, Peter Bäuerle, and Denis Andrienko. Comparative Study of Microscopic Charge Dynamics in Crystalline Acceptor-Substituted Oligothiophenes. J. Am. Chem. Soc., 134(13):6052-6056, 2012.

[157] Germà Garcia-Belmonte and Juan Bisquert. Open-circuit voltage limit caused by recombination through tail states in bulk heterojunction polymer-fullerene solar cells. Appl. Phys. Lett., 96(11):113301, 2010.

[158] Johannes Widmer, Max Tietze, Karl Leo, and Moritz Riede. Open-Circuit Voltage and Effective Gap of Organic Solar Cells. Adv. Funct. Mater., 23(46):5814-5821, 2013.

[159] L. J. A. Koster, V. D. Mihailetchi, R. Ramaker, and P. W. M. Blom. Light intensity dependence of open-circuit voltage of polymer:fullerene solar cells. Appl. Phys. Lett., 86(12):123509, 2005.

[160] William J. Potscavage, Asha Sharma, and Bernard Kippelen. Critical Interfaces in Organic Solar Cells and Their Influence on the Open-Circuit Voltage. Accounts Chem. Res., 42(11):1758-1767, 2009. 
[161] Koen Vandewal, Kristofer Tvingstedt, Abay Gadisa, Olle Inganäs, and Jean V. Manca. On the origin of the open-circuit voltage of polymer-fullerene solar cells. Nat. Mater., 8(11):904-909, 2009.

[162] Andrea Maurano, Rick Hamilton, Chris G. Shuttle, Amy M. Ballantyne, Jenny Nelson, Brian O'Regan, Weimin Zhang, Iain McCulloch, Hamed Azimi, Mauro Morana, Christoph J. Brabec, and James R. Durrant. Recombination Dynamics as a Key Determinant of Open Circuit Voltage in Organic Bulk Heterojunction Solar Cells: A Comparison of Four Different Donor Polymers. Adv. Mater., 22(44):4987-4992, 2010.

[163] Edward J. W. Crossland, Kim Tremel, Florian Fischer, Khosrow Rahimi, Günter Reiter, Ullrich Steiner, and Sabine Ludwigs. Anisotropic Charge Transport in Spherulitic Poly(3-hexylthiophene) Films. Adv. Mater., 24(6):839-844, 2012.

[164] Stephanie S. Lee, Jeffrey M. Mativetsky, Marsha A. Loth, John E. Anthony, and YuehLin Loo. Quantifying Resistances across Nanoscale Low- and High-Angle Interspherulite Boundaries in Solution-Processed Organic Semiconductor Thin Films. ACS Nano, 6 (11):9879-9886, 2012.

[165] Jonathan Rivnay, Leslie H. Jimison, John E. Northrup, Michael F. Toney, Rodrigo Noriega, Shaofeng Lu, Tobin J. Marks, Antonio Facchetti, and Alberto Salleo. Large modulation of carrier transport by grain-boundary molecular packing and microstructure in organic thin films. Nat. Mater., 8(12):952-958, 2009.

[166] Antti Ojala, Andreas Petersen, Andreas Fuchs, Robert Lovrinčić, Carl Pölking, Jens Trollmann, Jaehyung Hwang, Christian Lennartz, Helmut Reichelt, Hans Wolfgang Höffken, Annemarie Pucci, Peter Erk, Thomas Kirchartz, and Frank Würthner. Merocyanine/C60 Planar Heterojunction Solar Cells: Effect of Dye Orientation on Exciton Dissociation and Solar Cell Performance. Adv. Funct. Mater., 22(1):86-96, 2012.

[167] Robert Lovrinčić, Jens Trollmann, Carl Pölking, Jan Schöneboom, Christian Lennartz, and Annemarie Pucci. Orientation of Nonplanar Molecules in Polycrystalline Layers from Infrared Spectra: Core-Chlorinated Naphthalene Tetracarboxylic Diimides. $J$. Phys. Chem. C, 116(9):5757-5763, 2012.

[168] Martin Pfeiffer, Christian Uhrich, Annette Petrich, Rico Schuppel, Kerstin Schulze, Karl Leo, Peter Bäuerle, Egon Reinold, and Eduard Brier. United States Patent: 8426727 Organic photoactive device, 2013.

[169] Sam-Shajing Sun and Larry R. Dalton, editors. Introduction to organic electronic and optoelectronic materials and devices. Number 133 in Optical science and engineering. CRC Press, Boca Raton, 2008. ISBN 9780849392849. 
[170] Tatsuo Mori, Hyeong-Gweon Kim, Teruyoshi Mizutani, and Duck-Chool Lee. Electroluminescent Properties in Organic Light-Emitting Diode Doped with Two Guest Dyes. Jpn. J. Appl. Phys., 40(Part 1, No. 9A):5346-5349, 2001.

[171] Rodrigo Noriega, Jonathan Rivnay, Koen Vandewal, Felix P. V. Koch, Natalie Stingelin, Paul Smith, Michael F. Toney, and Alberto Salleo. A general relationship between disorder, aggregation and charge transport in conjugated polymers. Nat. Mater., 12 (11):1038-1044, 2013.

[172] Carl Poelking, Eunkyung Cho, Alexander Malafeev, Viktor Ivanov, Kurt Kremer, Chad Risko, Jean-Luc Brédas, and Denis Andrienko. Characterization of ChargeCarrier Transport in Semicrystalline Polymers: Electronic Couplings, Site Energies, and Charge-Carrier Dynamics in Poly(bithiophene-alt-thienothiophene) [PBTTT]. J. Phys. Chem. C, 117(4):1633-1640, 2013.

[173] Carl Poelking, Kostas Daoulas, Alessandro Troisi, and Denis Andrienko. Morphology and Charge Transport in P3ht: A Theorist's Perspective. In P3HT Revisited - From Molecular Scale to Solar Cell Devices, volume 265, pages 139-180. Springer Berlin Heidelberg, Berlin, Heidelberg, 2014. ISBN 978-3-662-45145-8.

[174] Amy M. Ballantyne, Lichun Chen, Justin Dane, Thomas Hammant, Felix M. Braun, Martin Heeney, Warren Duffy, Iain McCulloch, Donal D. C. Bradley, and Jenny Nelson. The Effect of Poly(3-hexylthiophene) Molecular Weight on Charge Transport and the Performance of Polymer:Fullerene Solar Cells. Adv. Funct. Mater., 18(16):2373-2380, 2008.

[175] R. Joseph Kline, Michael D. McGehee, Ekaterina N. Kadnikova, Jinsong Liu, Jean M. J. Fréchet, and Michael F. Toney. Dependence of Regioregular Poly(3-hexylthiophene) Film Morphology and Field-Effect Mobility on Molecular Weight. Macromolecules, 38 (8):3312-3319, 2005.

[176] A. Zen, J. Pflaum, S. Hirschmann, W. Zhuang, F. Jaiser, U. Asawapirom, J. P. Rabe, U. Scherf, and D. Neher. Effect of Molecular Weight and Annealing of Poly(3hexylthiophene) on the Performance of Organic Field-Effect Transistors. Adv. Funct. Mater., 14(8):757-764, 2004.

[177] Ala'a F. Eftaiha, Jon-Paul Sun, Ian G. Hill, and Gregory C. Welch. Recent advances of non-fullerene, small molecular acceptors for solution processed bulk heterojunction solar cells. Journal of Materials Chemistry A, 2(5):1201, 2014.

[178] Prashant Sonar, Jacelyn Pui Fong Lim, and Khai Leok Chan. Organic non-fullerene acceptors for organic photovoltaics. Energy \& Environmental Science, 4(5):1558, 2011. 
[179] Akshay Rao, Mark W. B. Wilson, Justin M. Hodgkiss, Sebastian Albert-Seifried, Heinz Bässler, and Richard H. Friend. Exciton Fission and Charge Generation via Triplet Excitons in Pentacene/C 60 Bilayers. J. Am. Chem. Soc., 132(36):12698-12703, 2010.

[180] Shane R. Yost, Jiye Lee, Mark W. B. Wilson, Tony Wu, David P. McMahon, Rebecca R. Parkhurst, Nicholas J. Thompson, Daniel N. Congreve, Akshay Rao, Kerr Johnson, Matthew Y. Sfeir, Moungi G. Bawendi, Timothy M. Swager, Richard H. Friend, Marc A. Baldo, and Troy Van Voorhis. A transferable model for singlet-fission kinetics. Nature Chemistry, 6(6):492-497, 2014.

[181] M. Dolores Perez, Carsten Borek, Stephen R. Forrest, and Mark E. Thompson. Molecular and Morphological Influences on the Open-Circuit Voltages of Organic Photovoltaic Devices. J. Am. Chem. Soc., 131(26):9281-9286, 2009.

[182] Eric T. Hoke, Koen Vandewal, Jonathan A. Bartelt, William R. Mateker, Jessica D. Douglas, Rodrigo Noriega, Kenneth R. Graham, Jean M. J. Fréchet, Alberto Salleo, and Michael D. McGehee. Recombination in Polymer:Fullerene Solar Cells with OpenCircuit Voltages Approaching and Exceeding 1.0 V. Advanced Energy Materials, 3(2): 220-230, 2013.

[183] Soon Ok Jeon, Kyoung Soo Yook, Byung Doo Chin, Yong Sup Park, and Jun Yeob Lee. Improved open-circuit voltage and efficiency in organic solar cells using a phosphine oxide based interlayer material. Solar Energy Materials and Solar Cells, 94(8):1389$1392,2010$.

[184] Hsiang-Yu Chen, Jianhui Hou, Shaoqing Zhang, Yongye Liang, Guanwen Yang, Yang Yang, Luping Yu, Yue Wu, and Gang Li. Polymer solar cells with enhanced open-circuit voltage and efficiency. Nat. Photonics, 3(11):649-653, 2009.

[185] Gabriele D’Avino, Sébastien Mothy, Luca Muccioli, Claudio Zannoni, Linjun Wang, Jérôme Cornil, David Beljonne, and Frédéric Castet. Energetics of Electron-Hole Separation at P3ht/PCBM Heterojunctions. J. Phys. Chem. C, 117(25):12981-12990, 2013.

[186] Koen Vandewal, Steve Albrecht, Eric T. Hoke, Kenneth R. Graham, Johannes Widmer, Jessica D. Douglas, Marcel Schubert, William R. Mateker, Jason T. Bloking, George F. Burkhard, Alan Sellinger, Jean M. J. Fréchet, Aram Amassian, Moritz K. Riede, Michael D. McGehee, Dieter Neher, and Alberto Salleo. Efficient charge generation by relaxed charge-transfer states at organic interfaces. Nat. Mater., 13(1):63-68, 2013.

[187] Françoise Provencher, Nicolas Bérubé, Anthony W. Parker, Gregory M. Greetham, Michael Towrie, Christoph Hellmann, Michel Côté, Natalie Stingelin, Carlos Silva, and Sophia C. Hayes. Direct observation of ultrafast long-range charge separation at polymer-fullerene heterojunctions. Nature Communications, 5, 2014. 
[188] Artem A. Bakulin, Akshay Rao, Vlad G. Pavelyev, Paul H. M. van Loosdrecht, Maxim S. Pshenichnikov, Dorota Niedzialek, Jérôme Cornil, David Beljonne, and Richard H. Friend. The Role of Driving Energy and Delocalized States for Charge Separation in Organic Semiconductors. Science, 335(6074):1340-1344, 2012.

[189] S. Gelinas, A. Rao, A. Kumar, S. L. Smith, A. W. Chin, J. Clark, T. S. van der Poll, G. C. Bazan, and R. H. Friend. Ultrafast Long-Range Charge Separation in Organic Semiconductor Photovoltaic Diodes. Science, 343(6170):512-516, 2014.

[190] Paul Westacott, John R. Tumbleston, Safa Shoaee, Sarah Fearn, James H. Bannock, James B. Gilchrist, Sandrine Heutz, John deMello, Martin Heeney, Harald Ade, James Durrant, David S. McPhail, and Natalie Stingelin. On the role of intermixed phases in organic photovoltaic blends. Energy \& Environmental Science, 6(9):2756, 2013.

[191] Fiona C. Jamieson, Ester Buchaca Domingo, Thomas McCarthy-Ward, Martin Heeney, Natalie Stingelin, and James R. Durrant. Fullerene crystallisation as a key driver of charge separation in polymer/fullerene bulk heterojunction solar cells. Chemical Science, 3(2):485, 2012.

[192] Chris Elschner, Manuel Schrader, Roland Fitzner, Alexandr A. Levin, Peter Bäuerle, Denis Andrienko, Karl Leo, and Moritz Riede. Molecular ordering and charge transport in a dicyanovinyl-substituted quaterthiophene thin film. RSC Advances, 3(30):12117, 2013.

[193] Timothy M. Burke and Michael D. McGehee. How High Local Charge-Carrier Mobility and an Energy Cascade in a Three-Phase Bulk Heterojunction Enable > 90\% Quantum Efficiency. Adv. Mater., 26(12):1923-1928, 2014.

[194] Zhi-Kuang Tan, Kerr Johnson, Yana Vaynzof, Artem A. Bakulin, Lay-Lay Chua, Peter K. H. Ho, and Richard H. Friend. Suppressing Recombination in Polymer Photovoltaic Devices via Energy-Level Cascades. Adv. Mater., 25(30):4131-4138, 2013.

[195] Björn Baumeier, Denis Andrienko, and Michael Rohlfing. Frenkel and Charge-Transfer Excitations in Donor-acceptor Complexes from Many-Body Green's Functions Theory. J. Chem. Theory. Comput., 8(8):2790-2795, 2012.

[196] Koen Vandewal. Photo-generation and recombination of charge carriers in organic solar cells. Conference presentation, ICSM (Turku), 2014.

[197] Samuel L. Smith and Alex W. Chin. Ultrafast charge separation and nongeminate electron-hole recombination in organic photovoltaics. Phys. Chem. Chem. Phys., 16 (38):20305-20309, 2014.

[198] Michael Schulze, Marc Hänsel, and Petra Tegeder. Hot Excitons Increase the Donor/Acceptor Charge Transfer Yield. J. Phys. Chem. C, 118(49):28527-28534, 2014. 
[199] James C. Blakesley and Dieter Neher. Relationship between energetic disorder and open-circuit voltage in bulk heterojunction organic solar cells. Phys. Rev. B, 84(7), 2011.

[200] Nico Christ, Siegfried W. Kettlitz, Sebastian Valouch, Jan Mescher, Mirco Nintz, and Uli Lemmer. Intensity-dependent but temperature-independent charge-carrier generation in organic photodiodes and solar cells. Organic Electronics, 14(3):973-978, 2013.

[201] Christian Koerner, Hannah Ziehlke, Roland Gresser, Roland Fitzner, Egon Reinold, Peter Bäuerle, Karl Leo, and Moritz Riede. Temperature Activation of the Photoinduced Charge-Carrier Generation Efficiency in Quaterthiophene: $\mathrm{C}_{60}$ Mixed Films. J. Phys. Chem. C, 116(47):25097-25105, 2012.

[202] John R. Tumbleston, Brian A. Collins, Liqiang Yang, Andrew C. Stuart, Eliot Gann, Wei Ma, Wei You, and Harald Ade. The influence of molecular orientation on organic bulk heterojunction solar cells. Nat. Photonics, 8(5):385-391, 2014.

[203] Sandeep Tyagi, Axel Arnold, and Christian Holm. Electrostatic layer correction with image charges: A linear scaling method to treat slab $2 \mathrm{~d}+\mathrm{h}$ systems with dielectric interfaces. J. Chem. Phys., 129(20):204102, 2008.

[204] Linjun Wang and David Beljonne. Flexible Surface Hopping Approach to Model the Crossover from Hopping to Band-like Transport in Organic Crystals. J. Phys. Chem. Lett., 4(11):1888-1894, 2013.

[205] A new multiscale modeling method for simulating the loss processes in polymer solar cell nanodevices. J. Chem. Phys., 136(19):194102, 2012. 